\title{
Mechanical Properties of Icosahedral Viral Shells. \\ A Molecular Dynamics Study
}

\author{
Dissertation \\ zur Erlangung des Doktorgrades \\ der mathematisch-naturwissenschaftlichen Fakultäten \\ der Georg-August-Universität zu Göttingen
}

\author{
Vorgelegt von \\ Mareike Zink \\ aus Osterholz-Scharmbeck
}

Göttingen, 2009 
D7

Referent: Prof. Dr. Helmut Grubmüller

Korreferent: Prof. Dr. Reiner Kirchheim

Tag der mündlichen Prüfung: 16.03.2009 


\section{Contents}

1 Introduction $\quad 1$

2 Virus architecture - an overview $\quad 7$

3 Computational Methods $\quad 13$

3.1 Molecular dynamics simulations . . . . . . . . . . . . . . . . . 13

3.1 .1 Integration method . . . . . . . . . . . . . . 16

3.1.2 Temperature and pressure coupling . . . . . . . . . . 16

3.1.3 Periodic boundary conditions and simulation box geometry . . 17

3.1.4 Simulation setup . . . . . . . . . . . . . . . . 18

3.2 Force-probe molecular dynamics simulations . . . . . . . . . . . . . . 19

3.3 Analysis . . . . . . . . . . . . . . . . . . . . 23

3.4 Scaling behavior on parallel machines . . . . . . . . . . . . . . . 24

4 The mechanical properties of Southern Bean Mosaic Virus 25

4.1 Introduction to Southern Bean Mosaic Virus . . . . . . . . . . . . . . 25

4.2 Simulation details . . . . . . . . . . . . . . . . . . . 28

4.2.1 Molecular dynamics simulations . . . . . . . . . . . . . 28

4.2.2 Force-probe molecular dynamics simulations . . . . . . . . . . 29

4.3 Results . . . . . . . . . . . . . . . . . . 31

4.3.1 Energy minimization and equilibration of the simulation system 31

4.3.2 Deformation of the capsid . . . . . . . . . . . . 37

4.3.3 Elastic constants . . . . . . . . . . . . . . . 38

4.3 .4 Yielding forces . . . . . . . . . . . . . . . . . 42

4.4 Discussion . . . . . . . . . . . . . . . . . . 47

4.5 Conclusion . . . . . . . . . . . . . . . . . . 50

5 Structural dynamics of SBMV during deformation 51

5.1 Introduction . . . . . . . . . . . . . . . . . . 51

5.2 Methods . . . . . . . . . . . . . . . . . . 52

5.3 Results and Discussions . . . . . . . . . . . . . . 53

5.3.1 Regimes of deformation . . . . . . . . . . . 53

5.3 .2 Water permeation, friction and surface effects . . . . . . . 54

5.3.3 Structural characterization of deformation . . . . . . . . . 58

5.3.4 Cross-over from elastic to plastic behavior . . . . . . . . . 65

5.3.5 Influence of the tip-sphere size . . . . . . . . . . . . . 70

5.3 .6 Influence of probe-velocity . . . . . . . . . . . . . . 73 
5.3.7 Influence of probe-velocity — Monte Carlo simulations . . . . 77

5.4 Conclusions . . . . . . . . . . . . . . . . . . . . 83

6 The mechanical properties of Human Rhinovirus $\mathbf{1 6} \mathbf{8 5}$

6.1 Introduction . . . . . . . . . . . . . . . . . . . 85

6.2 Simulation details . . . . . . . . . . . . . . . . . . . 90

6.2.1 Molecular dynamics simulations . . . . . . . . . . . . . . 90

6.2.2 Force-probe molecular dynamics simulations . . . . . . . . . . 91

6.2.3 Force field parameters for the fatty acid DAO . . . . . . . . . 92

6.3 Results . . . . . . . . . . . . . . . . . . . . . . . 94

6.3 .1 Equilibration . . . . . . . . . . . . . . . . 94

6.3.2 Deformation of the capsids . . . . . . . . . . . . . . . 97

6.3.3 Elastic constants . . . . . . . . . . . . . . . . . 97

6.3.4 Yielding forces . . . . . . . . . . . . . . 103

6.3.5 Structural changes during deformation . . . . . . . . . . . 107

6.4 Discussion . . . . . . . . . . . . . . . . . . . . . . . . . 122

7 Deformation of the globular titin kinase protein domain 127

7.1 Introduction . . . . . . . . . . . . . . . . . . . . 127

7.2 Methods . . . . . . . . . . . . . . . . . 127

7.3 Results . . . . . . . . . . . . . . . . . . . . . 128

7.4 Discussion . . . . . . . . . . . . . . . . . . 130

8 Summary and Conclusions 133

$\begin{array}{lll}9 & \text { Outlook } & 139\end{array}$

$\begin{array}{ll}\text { Acknowledgments } & 141\end{array}$

$\begin{array}{ll}\text { Bibliography } & 143\end{array}$ 


\section{Introduction}

Viral capsids are self-assembled nano-structures consisting of a protein shell to protect the genetic material inside [1,2]. The majority of plant viruses [3] and also lots of animal viruses are double or single-stranded RNA viruses that assemble spontaneously under in vitro conditions [4-6]. In contrast, most bacteriophages, viruses that infect bacteria, have a "molecular motor" (head-tail connector) attached to the viral shell which transports the DNA inside the viral shell after the self-assembly of the complete shell protein.

Recent experiments [7, 8] and theoretical investigations [9-11] have revealed highly organized DNA structures within fully loaded bacteriophages. Ejection forces exhibited from the head-tail connector and osmotic pressures of the confined DNA inside the viral particle were quantified to be of the order of $50 \mathrm{~atm}$ [12-14]. The high packaging density has been recognized as one of the key factors responsible for the enormous pressure caused by the DNA inside fully packed bacteriophages, whereas electrostatic repulsion has also been proposed to play an important role as well [15].

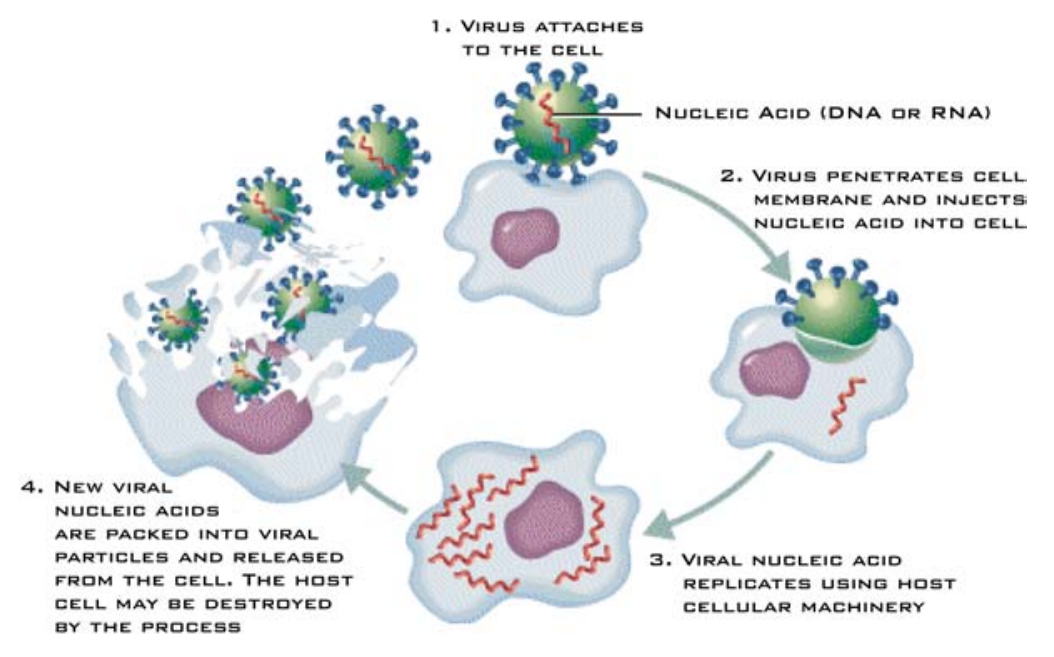

Figure 1.1: Schematic drawing of the viral replication cycle (taken from [16]).

Most DNA and RNA virus shells exhibit an icosahedral symmetry, whereas the function of the viral capsid and its mechanical properties are some of the main questions in medical research like drug design and biological, chemical and also physical science $[17,18]$. One of the major challenges in the approach of an improved picture of viral assembly and maturation [19] is a fundamental knowledge of the shell elastic properties and their distribution on the viral surface. 
How can an arrangement of proteins show a high stiffness and simultaneously behaves highly elastic [14]? Only an understanding of the mechanical properties can improve the function of viral shells as drug containers or the development of antiviral drug components and vaccines [20,21]. Furthermore, to study if and to what extent stiffness parameters such as elastic constants and Young's modulus as well as fracture behavior are distributed on the viral surface [22], is important in the process of viral infection during which the genetic material is injected into a cell (Fig. 1.1, step 2 ). Because, before the genetic material is released from the capsid, a transition of the mature viral shell to a structure which is able to release the DNA/RNA proceeds, in some cases observed as swelling of the capsid [23, 24]. A uniform swelling of the capsid with subsequent bursting of the viral shell was proposed by Tama et al. as a possible scenario for RNA release [25, 26]. Swelling is accompanied by a subsequent change of mechanical properties of the capsid which, in contrast to bursting of the complete shell, allows an opening of a port on the viral surface to release the genetic material.

For a possible scenario of RNA/DNA release, an opening of structural units of the viral shell, called capsomers (see chapter 2) has been suggested, in which the genetic material would leave the capsid along the symmetry axis [27, 28]. But until now the function of these two different capsomer geometries - pentamers around the 5-fold symmetry axis, and hexamers around the 3-fold symmetry axis - during viral maturation [29] and cell infection remains unclear [30]. One of the major challenges in understanding structural and dynamical differences of hexamers and pentamer, is the investigation of their mechanical properties [31]. The knowledge of the distribution of elastic constants and rupture forces on the capsomer surfaces with atomic resolution will lead to an improved picture of viral assembly, shell fracture and RNA/DNA release.

The development of atomic force microscopy (AFM) [32] offered the opportunity to investigate the mechanical properties of viral capsids, viral RNA and other biological systems on length scales of $10-30 \mathrm{~nm}[33,34]$. As a first step, this technique was applied to image viruses and measure protein-protein interactions [35-41]. In 1997, one of the first AFM experiments on viral coat proteins was performed by Falvo et al. [42] to investigate the elastic properties of tobacco mosaic virus. They determined a Young's modulus of $\sim 1 \mathrm{GPa}$ and compared this to macromolecular structures such as microtubules.

A more detailed picture of mechanical properties was given by Ivanovska et al. in 2004. The elastic response of the empty prohead of the bacteriophage $\phi 29$ during nano-indentation with an AFM tip was reported [43]. A linear response was obtained when the AFM tip was pushed into the capsid; for indentation $<30 \%$ of capsid height the linear regime was found to be reversible. Higher indentations caused a fracture of the viral shell and a rapid force decline. A bimodal distribution of elastic constants was found with peaks at $0.18 \mathrm{~N} / \mathrm{m}$ and $0.3 \mathrm{~N} / \mathrm{m}$. The maximum forces before fracture occurred were measured at $2.5 \mathrm{nN}$.

Similar observations were made by Michel et al. in 2006 from AFM experiments on 
full and empty cowpea chlorotic mottle virus (CCMV). The bimodal distribution of elastic constants showed values of the same order of magnitude as for $\phi 29$. Only the maximum forces were much smaller $(\sim 0.6 \mathrm{nN})$. Michel et al. pointed out that the elastic response might vary between hexamers and pentamers which could result in a bimodal distribution of elastic constants. But they were the first to question this explanation and asked if the resolution of the tip-sphere is high enough to obtain mechanical properties of viral shells on capsomer or even smaller length scales and if the bimodal distribution found in their and other groups' work is actually a property of viral shells rather than an anecdotal result.

In the same year, Carrasco et al. obtained contradicting results, taken from AFM experiments with full and empty minute virus of mice (MVM), an icosahedral DNA virus [44]. The stiffness of the empty capsid was reported to be isotropic, whereas the presence of DNA inside the virion led to an anisotropic reinforcement of the virus stiffness with respect to the 2-, 3- and 5-fold symmetry axes. The elastic constants and maximum forces for empty capsids were $0.15 \mathrm{~N} / \mathrm{m}$ and $0.60 \mathrm{nN}$ and for full capsids $0.20 \mathrm{~N} / \mathrm{m}$ and $0.81 \mathrm{nN}$ in average. They concluded that full capsids required a higher maximum force for irreversible deformation, whereas the anisotropic distribution of elastic properties might be caused by binding of the DNA to the internal capsid surface.

Ivanovska et al. continued with their work on mechanical properties of bacteriophages and published AFM experiments on WT phage in 2007 [14]. They showed that phages with WT DNA were twice as stiff as mutants with shorter DNA which behaved like empty capsids. A comparison to Falvo et al. [42] and their AFM experiments on tobacco mosaic virus (TMV) was reported by Schmatulla et al. in 2007 [45]. This group also carried out AFM experiments with TMV and measured elastic constants that were five times higher as all reported values before. How these discrepancies can be understood is still under discussion.

Furthermore, atomic force microscopy has been used to measure the effect of maturation and dematuration on the mechanical properties of viral capsids like murine leukemia virus (MLV) particles [29] and human immunodeficiency virus (HIV) [46]. Changes in the shell elasticity were found, but the question how and where the genetic material leaves the shell during cell infection could not be answered.

All these studies have shed more light on the distribution of mechanical properties on the outer surface of viral shells. Nevertheless, an atomistic picture is still missing and the differences in the elastic behavior of pentamers and hexamers and their function in viral assembly and cell infection remain unexplained.

Besides AFM experiments, theoretical studies addressed the questions

- How do the mechanical properties vary between full and empty capsids, and what are the structural determinants?

- How and why do the mechanical properties differ along the 2-, 3- and 5-fold axes for icosahedral viral shells? 
- Do the mechanical properties differ for pentamers and hexamers?

- What role do the mechanical properties play in cell infection; is there a "port" that can open to release the genetic material?

Tama et al. [25, 26], using elastic network normal mode analysis, came to the conclusion that pentamers are more flexible and their amino acids have a higher propensity to move freely than hexamers. In contrast, Hespenheide et al. [47] proposed a higher stiffness of pentamer than hexamers from percolation rigidity calculation, as well as Zandi et al. [48], who determined local stresses and pressures from continuum modeling.

Recent continuum and coarse-grained modelings investigated the buckling of viral capsids under applied forces [22, 49]. Gompper et al. [50] obtained elastic deformations for small indentations and the buckling transitions at large capsid deformations which depend both strongly on the number of elementary building blocks, the shear and bending elasticity of the shell and the confining geometry.

All these theoretical approaches rest on the assumption that atomic detail can be neglected in the attempt to quantify and explain the mechanical properties of viral shells. However, because the reaction of many proteins upon mechanical stress critically depends on atomic detail [51] and can e.g. be drastically altered by single point mutations [52], this assumption is questionable. Schulten et al. were the first to carry out all-atom equilibrium molecular dynamics simulations on the complete shell of tobacco mosaic virus [53]. But the sheer size of viral capsids has so far prevented molecular dynamics simulation to address the above questions at the atomic level by non-equilibrium studies of fully solvated viral shells.

One of the first molecular dynamics simulations of single proteins or subunits of viral capsids solvated in water, were reported by Phelps et al. in 1995 [54] and Speelman in 2001 [55]. They simulated a Human Rhinovirus 14 protomer with and without an antiviral compound and determined the flexibility of single amino acids. Furthermore, Yoneda et al. $[56,57]$ developed a computational method to simulate a rhinovirus capsid with rotational symmetry boundary conditions. With this method only a small part of rhinovirus, consisting of a few protomers, was simulated. The boundary conditions were chosen in a way that a complete spherical capsid was built up.

\section{Aim of the thesis}

In this work, the mechanical properties of the complete viral shell of Southern Bean Mosaic Virus (SBMV) and Human Rhinovirus (HRV) 16 solvated in water were studied with all-atom non-equilibrium force-probe molecular dynamics (FPMD) simulations $[58,59,51]$. SBMV is a prototypical RNA plant virus with $\mathrm{T}=3$ symmetry. Its capsid is composed of 60 symmetrical subunits, each containing three identical proteins (see chapter 4.1). The shell of the RNA rhinovirus 16 exhibits a pseudo $\mathrm{T}=3$ symmetry, with each of the 60 asymmetrical subunits (called protomers) containing four different proteins (chapter 6.1). Although remarkable differences in the 
assembly of both shells, SBMV and HRV 16 appear and result in different subunit structures, the different capsid proteins are folded into a similar eight-stranded antiparallel $\beta$-barrel motif with very similar positions and orientations of the $\mathrm{C}_{\alpha}$ atoms (chapter 6.1).

Characterization of the elastic properties of these elastic RNA viral shells should also shine light on the remarkable stability of DNA viral capsids, which have not been investigated with molecular dynamics simulations yet because of their huge size compared to RNA viruses. With a radius of $r \approx 16 \mathrm{~nm}$ the capsids of SBMV and HRV 16 are relatively small compared to other icosahedral RNA viruses, which rendered the extended FPMD simulations feasible. In total, the simulation systems contain over 4,500,000 particles and are therefore, to our best knowledge, the largest biomolecular systems simulated so far.

In the simulations, a Lennard-Jones sphere served as a model of a small and very sharp AFM tip which was pushed with a constant velocity into and through the capsid of SBMV and HRV. A tight grid of indentation positions was chosen in order to obtain a spatially resolved "elasticity map", whereupon this simulation technique offers the unique opportunity to investigate the mechanical properties of SBMV and HRV 16 on the outer and inner surface of the capsids.

Besides the FPMD simulations of the complete capsids of SBMV and HRV 16, also the mechanical properties of mutants of these shells were examined on atomic length scales. The removal of the 180 calcium ions included in the protein structure of SBMV is known to cause a swelling and thus, a destabilization of the capsid which becomes able to release the RNA [60]. In order to study possible changes in the distribution of elastic properties and fracture behavior on the shell of SBMV after calcium removal, additional force-probe simulations of SBMV were performed of the protein capsid without the calcium ions.

Hadfield et al. proposed that protein VP4, one of the four protomer proteins, and the pocket-factor included in protein VP1 of HRV 16 are key origins in destabilizing the capsid structure [61]. Also the removal of the zinc ions might result in variations of the mechanical properties as seen for SBMV. Until now it remains unclear how the destabilization can be understood and what changes in elastic properties occur. In contrast to SBMV, the mechanism of cell infection and the question if the removal of the pocket factor or protein VP4 results in changes of the capsid structure that becomes able to release the RNA has to be answered. To address these questions, force-probe simulations of three structural mutants of HRV 16 were performed: (1) Without protein VP4, (2) without the pocket-factor in protein VP1, (3) without zinc ions.

From the force-probe simulations of the complete shells of SBMV and HRV 16 and its capsid mutants, we aim at explaining possible pathways for RNA release due to variations of mechanical properties after mutation of the viral shells. Besides, structural changes during deformation of the capsids and the transition from elastic to plastic deformation shall be understood in structural terms. 


\section{Outline of the thesis}

This work is divided into eight parts. Chapter 2 gives an overview on viral architecture and characterization of viral structures. The employed molecular dynamics and force-probe simulation technique is described in chapter 3. The obtained elastic constants on the inner and outer capsid surface of Southern Bean Mosaic Virus and the effect of calcium removal are presented in detail in chapter 4. Structural changes of the SBMV capsid during deformation on length scales of the complete capsid and, additionally, on atomic level are discussed in chapter 5. Influences of the tip-sphere size and tip-sphere velocity are depicted and compared with recent experimental data. The explanation for the obtained velocity dependence required including nonequilibrium effects within the established theory of enforces barrier crossing. These assumptions are corroborated using Monte Carlo simulations. Chapter 6 focuses on the elastic constants and yielding forces obtained on different protein positions on the capsids of Human Rhinovirus 16 and three structural mutants. Two indentation simulations in which the tip-sphere was pushed along the 2- and 5-fold symmetry axes and the resulting structural changes are depicted and compared with results from the SBMV shell. Chapter 7 focuses on the deformation of the globular titin kinase protein domain. Force-probe simulation results were compared with the elastic behavior obtained from the capsid deformation of SBMV and HRV 16 and should give answers to the question how deformation of viral shells differ from the deformation of other proteins and why capsids are always considered as being extremely elastic. This work ends with a summary and conclusion (chapter 8) and gives an outlook to additional problems about the mechanical properties of viral shells and how the simulation technique can be improved to reach more direct comparison to AFM experiments (chapter 9). 


\section{Virus architecture - an overview}

All viruses exhibit common features and general principles of architecture despite the fact that there are specific requirements of each type of virus which resulted in a diversity of organization and geometrical design [62, 63]. The first assumptions about the shape of viruses and the geometry of the shell were made by Crick and Watson [64-66] in 1956 who proposed on the basis of theoretical considerations and of the weak experimental evidence available then, the following principles of virus structure that have been confirmed and universally accepted.

They first pointed out that the nucleic acid in small viruses was probably insufficient to code for more than a few protein molecules of limited size. The only reasonable way to build a protein shell, was to use the same type of molecule multiple times, leading to their theory of identical subunits. The second part of their proposal concerned the way in which the subunits must be arranged in the protein shell. Generally, it was expected that subunits would be packed so as to provide each of them with an identical environment. This is possible only if they are packed symmetrically. Crick and Watson pointed out that the only way to achieve this was to use some form of cubic symmetry, which possesses a number of axes around which it may be rotated to give a number of identical appearances. These predictions were soon confirmed and it became evident that the occurrence of icosahedral features in quite unrelated viruses was preferred in virus structure.

An icosahedron is composed of 20 facets, each an equilateral triangle, and 12 vertices, and because of the axes of rotational symmetry is said to have 5:3:2 symmetry. There are six 5-fold symmetry axes passing through the vertices, ten 3-fold axes extending through each face and fifteen 2-fold axes passing through the edges of an icosahedron (Fig. 2.1a).

Icosahedral symmetry requires defined numbers of structure units to complete a shell. In their discussion 1956, Crick and Watson [66], thinking in terms of asymmetrical protein subunits packed in such a way that each has an identical environment, pointed out that a virus with 5:3:2 symmetry requires a multiple of $\mathbf{6 0}$ subunits to cover the surface completely. Each unit would be connected identically and asymmetrically to its neighbors, and none of the units would coincide with a symmetry axis.

The introduction of negative staining [68] revolutionized the field of electron microscopy of viruses. Within just a few years, much new and exciting information about the architecture of virus particles was acquired. Not only were the overall shapes of particles revealed but also the symmetrical arrangement of their components. This led to a need for a new terminology to describe the viral components. In 1959, Lwoff, Anderson and Jacob [69] proposed the terms capsid and capsomers, 
a) Viral Shell, $T=1$

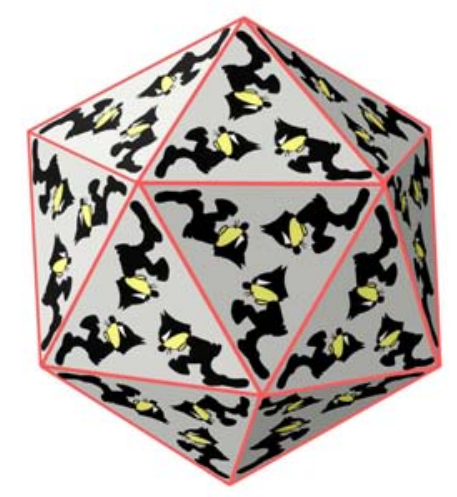

b) Symmetric Subunit

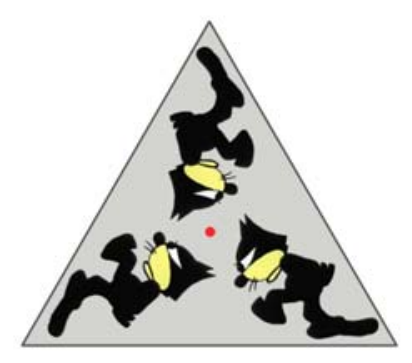

Figure 2.1: a) Viral shell with $\mathrm{T}=1$ symmetry - a perfect icosahedron consisting of 20 triangles with, in total, 60 copies of "Felix the cat". The heads point to the 3-fold symmetry axes, the paw to the 5-fold symmetry axes. b) Symmetric subunit - Three asymmetric proteins compose a symmetric subunit of a viral capsid, the red dot marks the rotational symmetry axis. (Pictures taken from [67].)

respectively, to represent the protein shell and the morphological units comprising it, and the term virion to denote the complete infective virus particle (i.e. a capsid enclosing the nucleic acid).

As soon as the first high resolution micrographs of negatively stained icosahedral viruses were obtained (1959: adenovirus [70], 1960: turnip yellow mosaic virus [71]) it seemed that there was a structural paradox. The number of morphological units (capsomers) observed on the surface of known icosahedral viruses at that time was never 60 or multiples of 60 , and was often more than 60 . Furthermore, the capsomers themselves appeared to be symmetrical and were located around symmetry axes.

There was direct evidence that capsomers of herpesvirus were hexagonal and pentagonal in section (Fig. 2.2). It is obvious that 5 -fold capsomers must be located on axes of 5-fold symmetry, and 6-fold capsomers may be situated on axes of 2-fold or 3 -fold symmetry, or in indifferent sites where they are suited to hexagonal packing. It was therefore clear that the capsomers were not equivalent to the subunits of Crick and Watson [66].

An obvious solution to the problem was provided by supposing that the symmetrical capsomers are built from a number of asymmetrical or symmetrical subunits, depending on the viral shell proteins. In this way it is possible to build a variety of complicated bodies in which 5:3:2 symmetry is preserved and in which the number of subunits is a multiple of 60 as predicted by Crick and Watson.

The theoretical basis for the structure of isometric viruses was put on a firm foundation by Caspar and Klug in 1962 with their concept of identical elements in quasiequivalent environments [73]. They defined all possible polyhedra in terms of structure units. The icosahedron itself has 20 equilateral triangular facets and therefore 


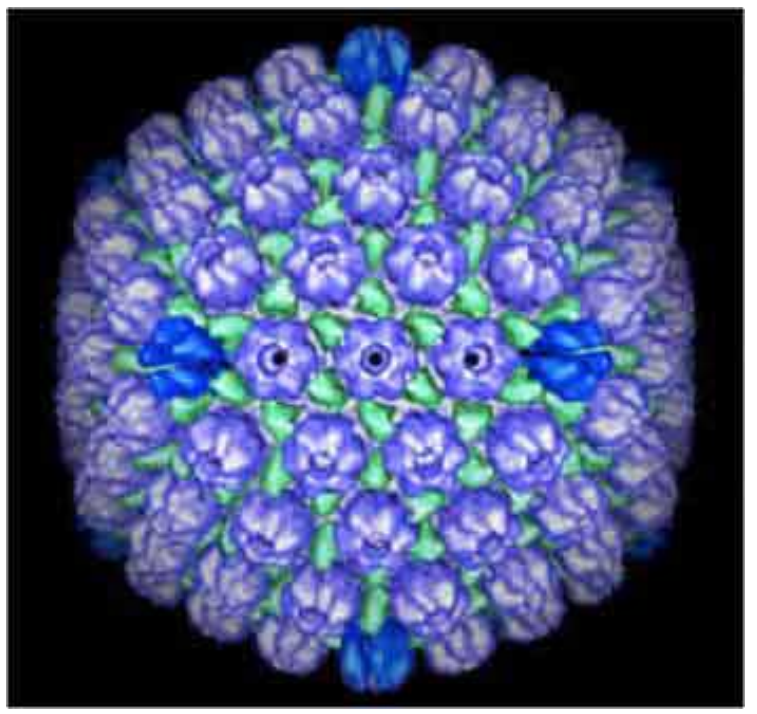

Figure 2.2: Cryo-transmission electron microscopy reconstruction of Herpesvirus, taken from [72].

$20 \mathrm{~T}$ structure units where $\mathrm{T}$ is the Triangulation number. The $\mathrm{T}$-number is an integer and calculated as $\mathrm{T}=h^{2}+k^{2}+h k$ ( $h$ : number of steps along lattice direction and $k$ steps along the other, with $h, k$ non-negative integers) which gives the number of equivalent sides in the resulting shell. For most of the common virus structures, the subunit is constructed from three proteins, whereas capsomers can be clustered as $20 \mathrm{~T}$ trimers, $30 \mathrm{~T}$ dimers or separated as $60 \mathrm{~T}$ monomers. The number of morphological units that would be produced by a clustering into hexamers and pentamers can be calculated as $10(\mathrm{~T}-1)$ hexamers plus 12 pentamers.

In a review of symmetry in virus architecture from 1961, Horne and Wildy showed that all the viruses then known (with the exception of a few bacteriophages) fell into two main morphological groups: (1) with cubic (icosahedral) symmetry and (2) with helical symmetry [74]. Other virions are composed of icosahedral "heads" mounted on a helical "tail" with tail fibers. The size of icosahedral viruses ranges from $30 \mathrm{~nm}$ to $50 \mathrm{~nm}$ in diameter. "Linear" viral capsids have RNA genomes that are encased in a helix of identical protein subunits. The length of the helical viral nucleocapsid is determined by the length of the nucleic acid, helical viruses can be as long as $1000 \mathrm{~nm}$. The tobacco mosaic virus [75], polio and rhinoviruses [76] are among the smallest viruses and the pox virus is among the largest animal viruses.

At the time when the viral morphology was studied, the architecture of most viral shells like the myxoviruses (influenca viruses) was poorly understood. Early electron micrographs of shadow-cast preparations revealed particles of varying shape and size but little detail could be reported [77]. With the advent of negative staining, it became obvious that the myxo- and paramyxoviruses consisted of an inner nucleo-protein component with helical symmetry surrounded by an envelope of characteristic morphology. This realization of the helical symmetry of the myxoviruses laid the foundation for the understanding of the symmetry of other complex groups 
of viruses such as rabies virus and granulosis virus [78].

In an attempt to clarify the terminology for virus components, in 1962 Caspar et al. [79] made a number of proposals which were generally accepted. Briefly, the proposals are as follows:

1. The CAPSID (also called coat protein) denotes the protein shell that encloses the nucleic acid. It is built of structure units.

2. STRUCTURE UNITS are the smallest functional equivalent building units of the capsid, thus individual proteins.

3. CAPSOMERS are morphological units seen on the surface of particles and represent clusters of structure units.

4. The capsid together with its enclosed nucleic acid is called the NUCLEOCAPSID.

5. The nucleocapsid may be embedded in an ENVELOPE which may contain material of host cell as well as viral origin.

6. The VIRION is the complete infective virus particle.

Nowaday, these proposals were confirmed using x-ray crystallography and nuclear magnetic resonance (NMR) and denote definitions of the viral components. Experiments revealed that most of the common plant and animal viral shells exhibit a $\mathrm{T}=3$ symmetry. They consist of three to four individual proteins, building the 60 symmetric or asymmetric subunits that again compose 20 hexamers and 12 pentamers. Tomato bushy stunt virus was the first icosahedral virus whose structure was determined on atomic resolution [80]. Subsequently, the structures of Southern Bean Mosaic Virus [81] and Satellite Tobacco Necrosis Virus [82] were determined and were found to have similar tertiary and quaternary structures. Later the crystal structure of some animal and human viral shells were resolved and compared. It turned out that the common structural folding motiv of most viral shell proteins such as the coat protein of Human Rhinovirus 14 and poliovirus, as well as of the mentioned RNA plant viruses are very similar [81]. 


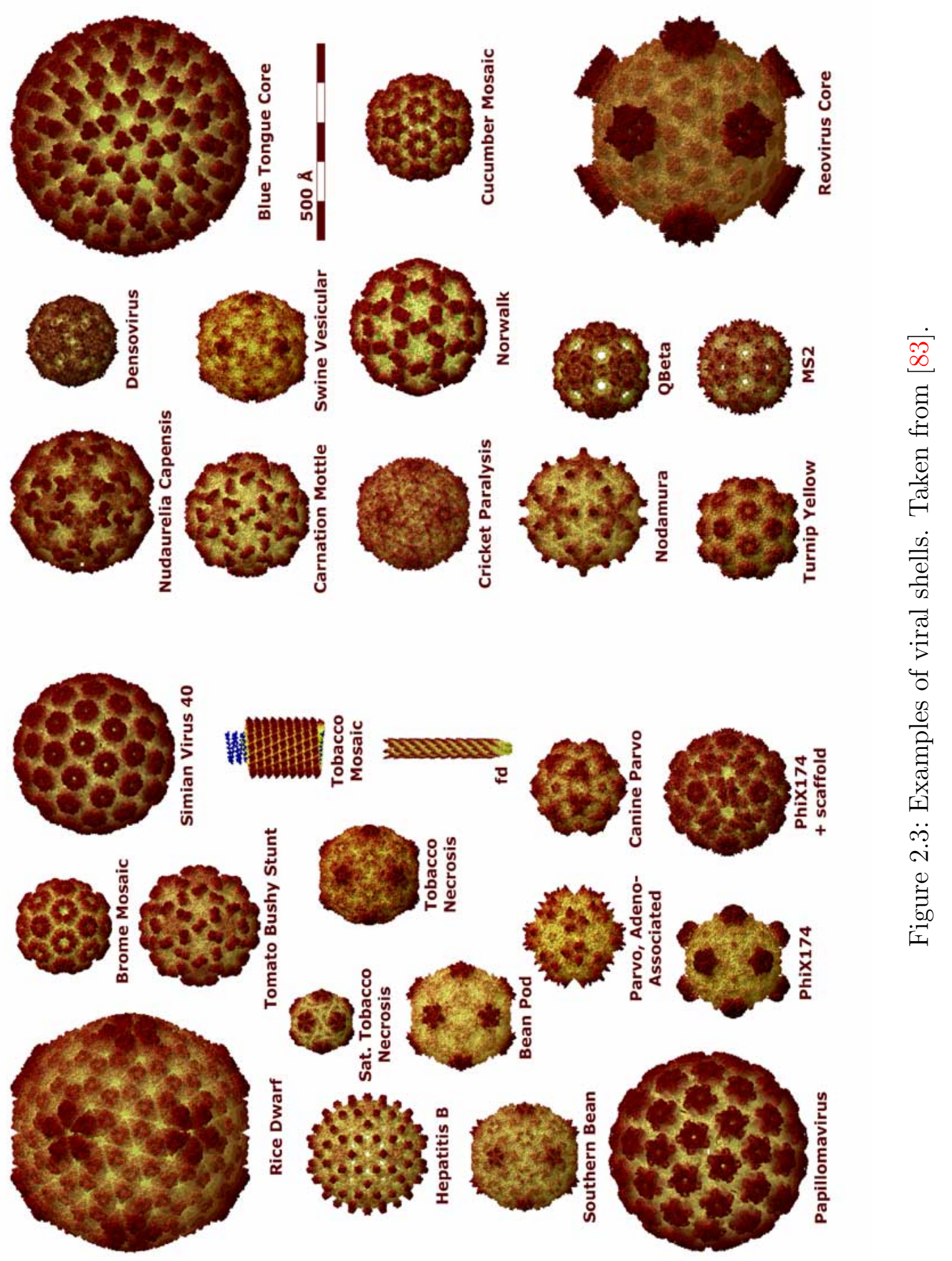




\section{Computational Methods}

In this chapter the principles of molecular dynamics (MD) simulations and forceprobe molecular dynamics (FPMD) simulations are introduced. General information is given for the underlying theory and assumptions, why quantum mechanics particles can be treated classically and how MD simulations work. The focus of this chapter lies on the description of the FPMD simulation technique which was the essential tool to compute the mechanical properties of viral shells from non-equilibrium MD simulations.

\subsection{Molecular dynamics simulations}

Molecular dynamics is a powerfull method to compute the dynamics of atoms and molecules on atomic length-scale and on time-scales of nano- to even microseconds - depending on the simulation system size, as well as on simulation parameters and force-fields. With modern computer power MD simulations of more than 1,000,000 atoms can be performed.

Three approximations are necessary to treat quantum mechanical particles with classical molecular dynamics methods:

\section{Born-Oppenheimer approximation}

The idea of the Born-Oppenheimer approximation [84] is the decoupling of the slow nucleic motion from the fast electron motion which is possible because of the much higher mass of the nuclei compared to the electrons. A system consisting of $M$ nuclei with positions $\mathbf{R}=\left\{\mathbf{R}_{1}, \ldots, \mathbf{R}_{M}\right\}$ and $N$ electrons at $\mathbf{r}=\left\{\mathbf{r}_{1}, \ldots, \mathbf{r}_{N}\right\}$ is described by the wave function $\psi_{m o l}=\psi_{m o l}(\mathbf{R}, \mathbf{r}, t)$. The approximation separates the wave function into an electron part $\psi_{e}(\mathbf{R}(t), \mathbf{r})$, depending on the electron and nucleic positions, and a nucleic term $\psi_{N}(\mathbf{R}(t))$ for the nucleic position and the time evolution:

$$
\psi_{m o l}(\mathbf{R}, \mathbf{r}, t)=\psi_{e}(\mathbf{R}, \mathbf{r}) \psi_{N}(\mathbf{R}, t) .
$$

Assuming that the positions of the nuclei are fixed and the electronic wave function depends only on the positions and not velocities of the nuclei, the Hamiltonian for the electrons and nuclei can be written as

$$
H=H_{e}+H_{N}
$$

with

$$
H_{e}=T_{e}+V_{e e}(\mathbf{r})+V_{e N}(\mathbf{R}, \mathbf{r})
$$




$$
H_{N}=T_{N}+V_{N N}(\mathbf{R})
$$

$H_{e}$ is the electron Hamiltonian that is the sum of the kinetic energy of the electrons $T_{e}$, the interaction between the electrons $V_{e e}$, and between electrons and nuclei $V_{N e}$. $H_{N}$ is the Hamiltonian of the nuclei that depends on the kinetic energy of the nuclei and their interactions $V_{N N}$.

The electron wave function is assumed to satisfy the time-independent Schrödinger equation,

$$
H_{e} \psi^{\alpha}(\mathbf{R}, \mathbf{r})=E_{e}^{\alpha}(\mathbf{R}) \psi_{e}^{\alpha}(\mathbf{R}, \mathbf{r}) .
$$

$E_{e}^{\alpha}(\mathbf{R})$ denotes the electronic energy for a quantum state $\alpha$ as a function of $\mathbf{R}$. The electronic wave function depends parametrically on the nuclear configuration $\mathbf{R}$ resulting in an effective potential for the nucleic motion. Usually the potential is given by the ground state energy $E_{e}(\mathbf{R})$.

The nuclear part of the wave function follows the time-dependent Schrödinger equation

$$
\left[H_{N}+E_{e}^{\alpha}(\mathbf{R})\right] \psi_{N}(\mathbf{R}, t)=i \hbar \frac{\partial \psi_{N}(\mathbf{R}, t)}{\partial t}
$$

Both Schrödinger equations 3.5 and 3.6 are not exact but it has been shown that they describe the motion of nuclei with very high accuracy $[85,86]$.

\section{Motion of the nuclei}

Most molecular dynamics simulation system contain thousands to millions of atoms for which it is impossible to solve the time-dependent Schrödinger equation 3.6. Therefore, the second approximation treats the nuclei as classical particles with a potential energy, called Born-Oppenheimer potential,

$$
V_{N}(\mathbf{R})=V_{N N}(\mathbf{R})+E_{e}^{\alpha}(\mathbf{R}) .
$$

With the potential energy surface of the ground state (eq. 3.5), the nuclei motion $V_{N}(\mathbf{R})=E_{e}^{0}(\mathbf{R})$ is described by Newton's equation of motion that describes the nuclei $i$ with mass $m_{i}$ as a classical point particle

$$
\begin{gathered}
m_{i} \frac{d^{2} \mathbf{R}_{i}}{d t^{2}}=-\frac{d}{d \mathbf{R}_{i}} V_{N}\left(\mathbf{R}_{i}\right) \\
m_{i} \mathbf{a}_{i}=\mathbf{F}_{i}
\end{gathered}
$$

where $\mathbf{F}_{i}$ is the force acting on nucleus $i$ and $\mathbf{a}_{i}$ its acceleration. Changes of the force within a discrete time step $\Delta t$ lead to a variation of the velocity and position of atom $i$ which can directly be derived from equation 3.9 by integration. 


\section{Force fields}

Solving the time-independent Schrödinger equation 3.5 for a simulation system of more than a few hundred atoms is still impossible due to the enormous computation time needed. Therefore a third approximation is required for the computation of the atom dynamics and to reduce computation time. For biological systems, consisting of amino acids and water molecules, the potential energy of the electrons $E_{e}(\mathbf{R})$ is approximated as a sum of semi-empirical functions that are easier to compute but still describe $E_{e}(\mathbf{R})$ in an appropriate way. Accordingly, the potential energy of all atoms $V(\mathbf{R})$, named force field, is expressed as

$$
V(\mathbf{R})=V_{B}+V_{a}+V_{i m p}+V_{D}+V_{q}+V_{v d W} .
$$

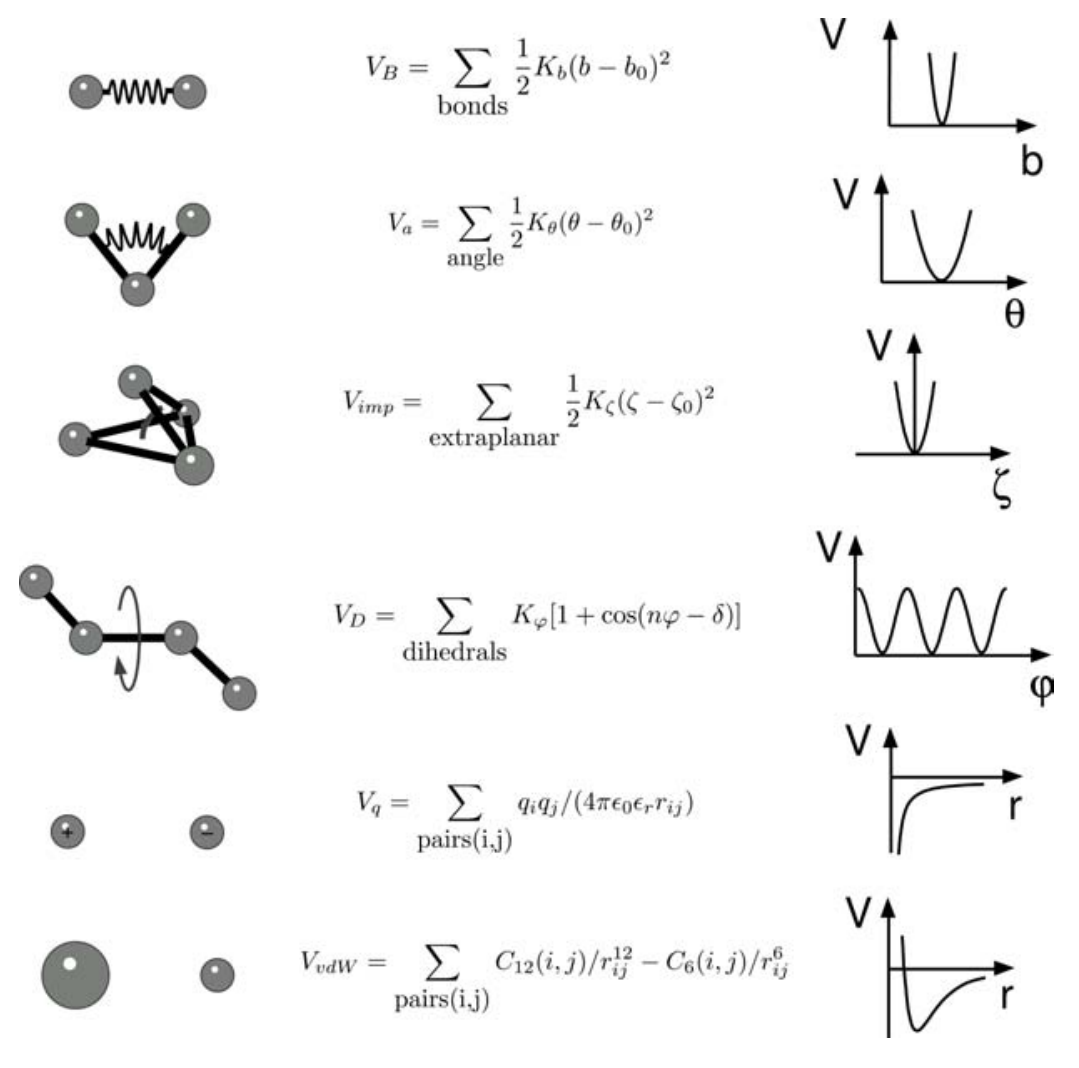

Figure 3.1: Schematic illustration of the force field terms for the potential energy calculation (picture by Gunnar Schröder).

The single terms of equation 3.10, depicted in Fig. 3.1, descibe the potential energy of the system given by the bonds between atoms, the angles, dihedral and improper dihedral angles, Coulomb and van-der-Waals interactions. The assumption here is that the atoms are connected via bonds based on a ball-and-spring model. All MD and MDFP simulations were performed using the OPLS all-atom force-field [87]. For more information on force fields, their differences and advantages see e.g. [88, 89]. 


\subsubsection{Integration method}

The dynamics of every atom in the simulation system is determined by integration of equation 3.9 for every particle, whereas an analytical solution is only possible for two or less particles. Therefore several numerical integration methods have been introduced. For the presented simulations the Verlet algorithm [90] was applied. The integration scheme is as follows:

$$
\begin{gathered}
\mathbf{v}_{i}\left(t+\frac{\Delta t}{2}\right)=\mathbf{v}_{i}\left(t-\frac{\Delta t}{2}\right)+\frac{\mathbf{F}_{i}(t)}{m_{i}} \Delta t \\
\mathbf{r}_{i}(t+\Delta t)=\mathbf{r}_{i}(t)+\mathbf{v}_{i}\left(t+\frac{\Delta t}{2}\right) \Delta t
\end{gathered}
$$

with the velocity $\mathbf{v}_{i}$, position $\mathbf{r}_{i}$ and force $\mathbf{F}_{i}$ on atom $i$. The integration time step has to be chosen to be smaller than the fastest motion, e.g. thermal fluctuations, in the system. Especially the light hydrogen atoms exhibit a bond vibration period of only a few femtoseconds and, thus, limit $\Delta t$ to $\sim 1.0$ fs to suppress artifacts. The LINCS algorithm [91] constrains the bond lengths which allows to increase the integration step size to $2.0 \mathrm{fs}$.

\subsubsection{Temperature and pressure coupling}

The temperature $T$ of the simulation system is defined by the kinetic energy $E_{k i n}$ and therefore by the velocity of the atoms $v$ with mass $m$ in the simulation box

$$
E_{k i n}=\sum_{i} \frac{1}{2} m v_{i}^{2}=\frac{f}{2} k_{B} T .
$$

$f$ is the number of degrees of freedom. At the beginning of the simulation, the velocities are chosen accordingly to a Maxwell distribution. One method to control the temperature is the Berendsen algorithm [92] which couples the system weakly to an external heat bath with given temperature $T_{0}$. The coupling strength is given by a coupling constant $\tau_{T}$, here $0.1 \mathrm{ps}$ for the force probe simulations. Accordingly, the velocity of every particle is rescaled every integration time step, with the new velocity $v^{\prime}$ given by

$$
v^{\prime}=v \sqrt{1+\frac{\Delta t}{\tau_{T}}\left(\frac{T_{0}}{T}-1\right)} .
$$

The idea of the Berendsen barostat for pressure control is similar. This algorithm rescales the box vectors and coordinates with respect to a coupling time $\tau_{p}$ and a given pressure $\mathbf{P}_{0}$. For isotropic pressure as used in the simulations, the scaling factor for the pressure $P$ is given by

$$
\mu=1-\frac{\Delta t}{3 \tau_{P}} \kappa\left(P_{0}-P\right),
$$


$\kappa$ is the isothermal compressibility of the system, which is $4.6 \cdot 10^{-5} \mathrm{bar}^{-1}$ for water at $1 \mathrm{~atm}$ and $300 \mathrm{~K}$. The pressure $P$ is calculated from the kinetic energy and the virials $\Xi$

$$
P=\frac{2}{3 V}(E-\Xi),
$$

with $V$ the volume of the simulation system. $\Xi$ is defined by the distance between atom $i$ and $j$ and the force $\mathbf{F}_{i j}$ acting between the atoms

$$
\Xi=-\frac{1}{2} \sum_{i<j}\left(\mathbf{r}_{i}-\mathbf{r}_{j}\right) \cdot \mathbf{F}_{i j} .
$$

\subsubsection{Periodic boundary conditions and simulation box geometry}

One difference between experiments and simulation is the size of the system of interest: Whereas in experiments a protein, e.g. a viral shell is solvated in a volume of some microliters of water, the simulation setup for a viral capsid in water is orders of magnitude smaller for a molecular dynamics simulation. On the one hand, the simulation system should contain as few atoms as possible to save computing time; on the other hand interactions between atoms should be as realistic as possible. In the present work, periodic boundary conditions (pbc) were chosen to avoid surfaces, as well as atomic interaction artifacts. The pbc copy the simulation box infinite times. If one atom moves out of the box at one side, it enters the box instantaneously at the opposite side. Problems might arise if the box size is chosen too small and the protein interacts with its periodic image. This could be prevented by a reasonably thick water layer around the protein (approx. $\sim 2.0 \mathrm{~nm}$, depending on the simulation parameters) to increase the distance between the protein and its replica.

For the simulations of SBMV and rhinovirus, the simulation boxes were chosen to have the shape of a truncated dodecahedron, which is a most regular space-filling unit-cell [93]. This box geometry is more spherical than a cube and the embedded volume is only $71 \%$ compared to a cube. It therefore safes about $29 \%$ of computing time [94] when simulating a spherical viral shell in a box of water because much fewer water molecules are required to fill up cavities in the box around the viral shell.

A periodic system with a rhombic dodecahedral shape is equivalent to a periodic system based on a space-filling triclinic unit cell because a rhombic dodecahedron is a Wigner-Seitz cell of a triclinic box [95]. The software package GROMACS, which was used for the simulations (see 3.1.4), is based on the triclinic unit cell. Thus, during the simulations the position of the viral shell proteins and the water molecules are calculated for a triclinic box (see Fig. 3.2). Although optically one could imagine that the viral capsid is not spherical anymore and some atoms are positioned at the wrong place in the triclinic box, the periodic boundary conditions replicate the copies of the cell in that way that the capsid is complete (Fig. 3.2). 


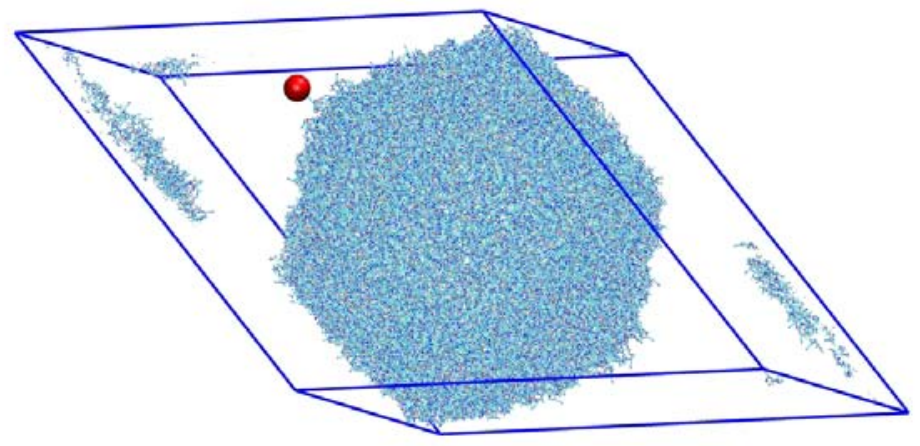

Figure 3.2: Triclinic simulation box. The viral shell of SBMV is shown in blue. In the triclinic box, the protein is not arranged in a compact structure, parts seem to be outside the box (the upper left and lower right corner of the box) due to the periodic boundary conditions. The tip-sphere is depicted by a red sphere.

\subsubsection{Simulation setup}

To set up the simulation system, the GROMACS-3.3.1 simulation software package [96] with the OPLS-AA force-field [87] and the TIP4P water model [97] was used. The x-ray structures of Southern Bean Mosaic Virus and Human Rhinovirus 16 where taken from the protein data bank (PDB) and VIPER data bank [98, 99]. After adding hydrogen atoms to the protein structures using WHAT IF [100] and the GROMACS tool pdb2gmx, the viral capsids were solvated in a rhombic dodecahedral box with box vector angles of $60^{\circ} \times 60^{\circ} \times 90^{\circ}$. The minimum distance to the boundaries and therefore the minimum thickness of the water shell around the capsids was chosen as $2.0 \mathrm{~nm}$. Water was also added inside the capsids because the structures of the two respective RNA strands are unknown and therefore cannot be modeled into the virus. Sodium and chloride ions were placed within the water according to a Debye-Hückel distribution governed by the protein charges, corresponding to a physiological ion condition of $150 \mathrm{mM}$. During all simulations, the protein and the solute were separately coupled to an external temperature bath with various coupling times $\tau_{T}$ (see section 4.2.1 and 6.2). A Berendsen barostat with $\tau_{p}=1.0 \mathrm{ps}$ and a compressibility of $4.5 \cdot 10^{-5} \mathrm{bar}^{-1}$ was used to keep the isotropic pressure constant at 1.0 bar. Lennard-Jones and van-der-Waals interactions were calculated within a cutoff distance of $0.9 \mathrm{~nm}$, whereas long-range electrostatic interactions were calculated using the particle-mesh Ewald method [101] with a grid spacing of $0.12 \mathrm{~nm}$. 


\subsection{Force-probe molecular dynamics simulations}

Force-probe molecular dynamics (FPMD) simulations are non-equilibrium simulations with the aim to deform a protein by applying an external force to the molecule to e.g. enforce protein unfolding or determine its elastic properties. All FPMD simulations were run in an NPT ensemble with $\tau_{T}=0.1 \mathrm{ps}$ and $\tau_{p}=1.0 \mathrm{ps}$. In contrast to the equilibration runs, the FPMD simulations were carried out with the GROMACS 4.0 CVS version from July $4^{\text {th }} 2007[102,103]$ for efficiency reasons. Newly implemented domain decomposition and dynamic load balancing reduced computation time for the present system by a factor of 10 compared to GROMACS-3.3.1.

To model the atomic force microscopy (AFM) tip, a Lennard-Jones sphere (LennardJones parameters $\sigma=5.0 \mathrm{~nm}$ and $\epsilon=0.001 \mathrm{~kJ} / \mathrm{mol}$ ) served as a model of a sharp tip (Fig. 3.3). During a force-probe simulation, this "tip-sphere" was pushed with a constant velocity towards and through the viral capsid (Fig. 3.4). The small tip size compared to AFM tips with typical diameters between 10-20 nm used for AFM experiments on viruses was chosen for the following reasons: On the one hand the diameter of the tip-sphere had to be smaller than the bulk water shell around the viral shell. On the other hand, a bigger tip-sphere would result in a larger cutoff distance for the van-der-Waals interactions during the simulation, whereas a doubling of the cutoff distances increases the computation time by a factor of two.

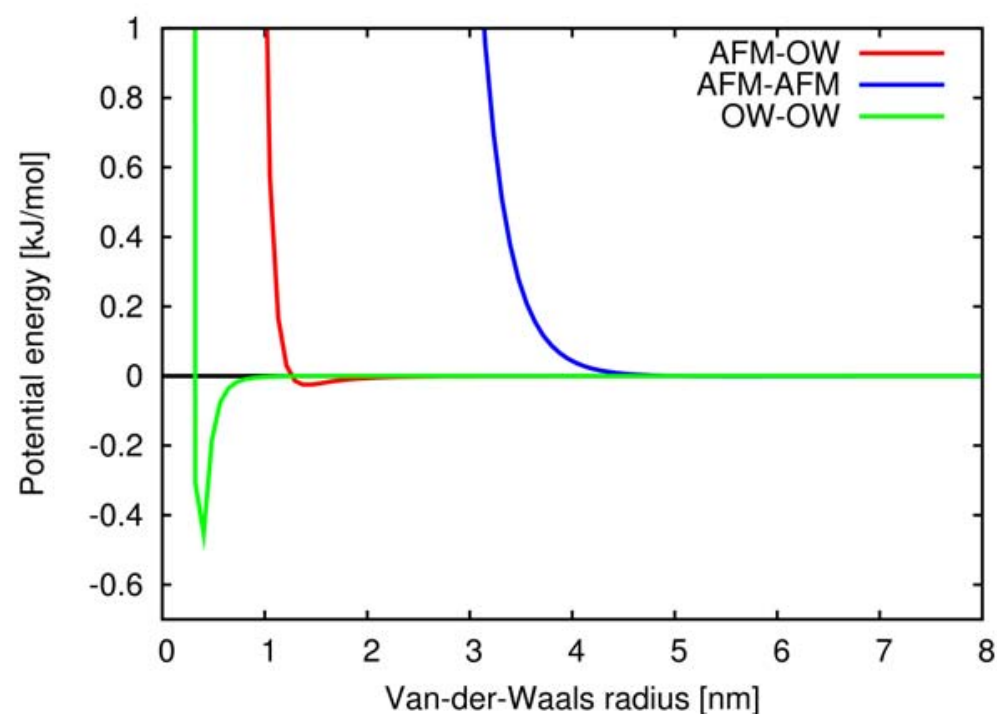

Figure 3.3: Lennard-Jones potential for the tip-sphere (labeled AFM) and an oxygen atom from the TIP4P water model (labeled OW) for interaction between two tip-spheres, two OW atoms and one AFM with one OW atom.

Although the equilibrium distance between the tip-sphere and the protein atoms was about $1.3 \mathrm{~nm}$, a cutoff for the van-der-Waals interaction of $0.9 \mathrm{~nm}$ did not cause artifacts as found from comparisons with larger cutoff distances. The potential energy 


\subsection{Force-probe molecular dynamics simulations}

corresponding to a distance of $0.9 \mathrm{~nm}$ was still small enough to enable a minimum distance between the tip-sphere and the surrounding atoms even smaller at $0.7 \mathrm{~nm}$ as found from a radial distribution function for the water atoms and the tip-sphere (see also section 5.3.2 and Fig. 5.2).

Furthermore, the diameter of the investigated viral capsid subunits was of the order of $7.0 \mathrm{~nm}$. Only a much smaller tip-sphere allows the investigation of mechanical properties on a tight grid as shown in Fig. 3.5 and on atomic length scales.

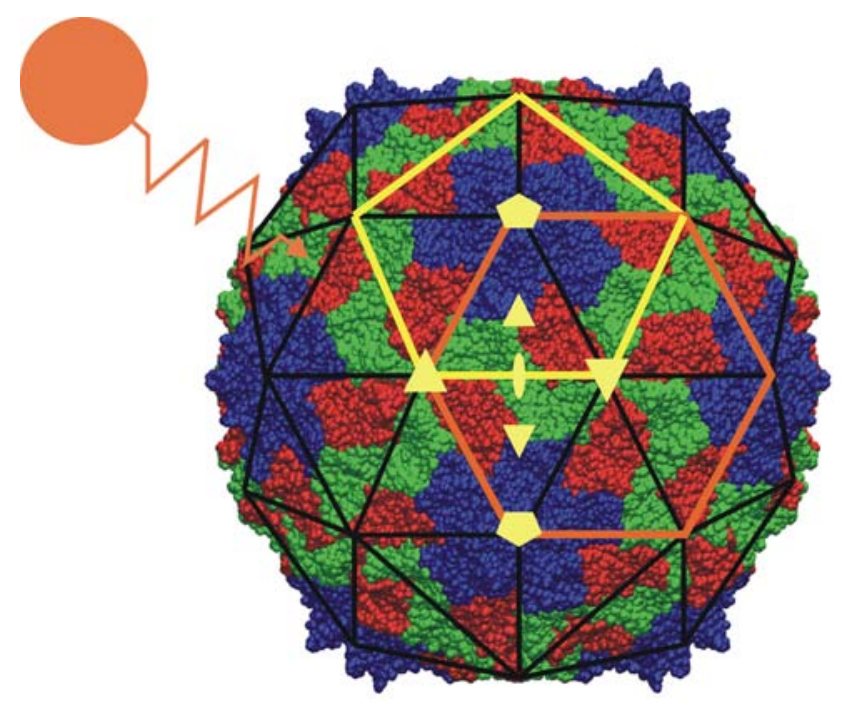

Figure 3.4: Southern Bean Mosaic Virus (SBMV) is built up from 60 subunits (black triangles), each composed of protein A (red), protein B (blue), protein C (green). The symbols denote the 5-, 3- and 2-fold symmetry axes. One pentamer is marked in yellow, one hexamer is emphasized with orange lines. The approaching tip-sphere (orange) is located close to the surface and attached to a "virtual" spring which pushes the tip-sphere against the viral shell. From the elongation of the spring, the force acting on the tip-sphere was determined.

In order to push the tip-sphere along a certain vector onto the viral surface, the tip-sphere was subjected to an isotropic harmonic potential,

$$
V_{\text {tip }}(t)=\frac{k}{2}\left(\mathbf{x}(t)-\mathbf{x}_{0}-\mathbf{v} \cdot t\right)^{2},
$$

the minimum of which was moved with a constant velocity $\mathbf{v}(t)$ towards the surface of the viral shell (Fig. 3.4). Here $k=1000 \mathrm{~kJ} /\left(\mathrm{mol} \mathrm{nm}{ }^{2}\right)$ is the spring constant, $\mathbf{x}$ is the current position of the tip-sphere and $\mathbf{x}_{0}$ its initial position.

The instantaneous force acting onto the shell was calculated from the force $\mathbf{F}_{\text {tip }}$ acting on the tip-sphere using Hooke's law, 


$$
\mathbf{F}_{t i p}(t)=-k\left(\mathbf{x}(t)-\mathbf{x}_{0}-\mathbf{v} \cdot t\right) .
$$

A force-time and force-distance curve was obtained from every force-probe simulation, in which a linear force increase depicted elastic behavior of the capsid during indentation with the tip-sphere.

To obtain an "elasticity map" of the viral surface, a total of 19 simulations was performed, each with the "tip" directed and moving towards a different grid point on the capsid surface (Fig. 3.5). The grid points were evenly distributed on the triangular surface of one of the 60 identical subunits.

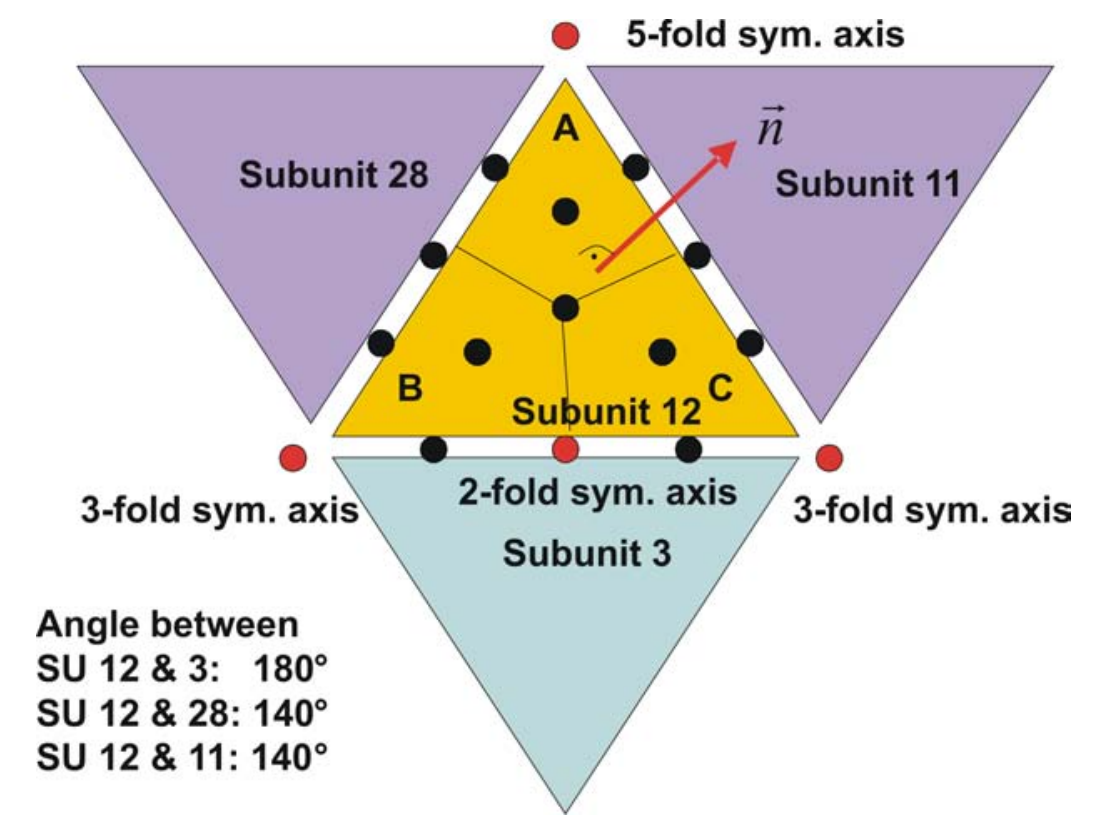

Figure 3.5: Schematic representation of subunit 12 (yellow) of Southern Bean Mosaic Virus and its adjacent subunits. The black dots mark the grid points on the capsid; the red dots emphasize the grid points at the 2-, 3- and 5 -fold symmetry axes. The normal vector $\vec{n}$ is given by the red arrow.

For each simulation the velocity vector was chosen to be perpendicular to the tangent through the grid point towards which the tip-sphere was pushed. Therefore, we assumed a planar subunit surface for Southern Bean Mosaic Virus and Human Rhinovirus 16. With this assumption, the vector along the 2-fold symmetry is equivalent to the vector perpendicular to the subunit surface and was employed as the "pushvector" in the respective simulations in which the tip-sphere was pushed against the subunit. When the tip-sphere was directed towards the center of the pentamer and hexamer, respectively, the vectors along the 5- and 3-fold symmetry axes, respectively, were chosen as the perpendicular vectors. At the subunit interfaces that connect the 5- and 3-fold symmetry axes (Fig. 3.5), vector addition of the vector perpendicular to subunit 12 of SBMV, and the perpendicular vector to subunit 11 and 28, respectively, determines the perpendicular vector to the interface of subunit 
11-12 and subunit 12-28 of SBMV. The same method was used to find the perpendicular vectors to the grid points on the surface of HRV 16.

To set up the FPMD simulation system, the tip-sphere was placed inside in the bulk water in the simulation box. Here, all water molecules within a radius of $0.7 \mathrm{~nm}$ - the minimum distance between the tip-sphere and the water atoms (Fig. 5.2) were removed to avoid overlap with the tip-sphere. The water molecules around the tip-sphere equilibrated within a few picoseconds.

In contrast to AFM experiments, FPMD simulations offer the opportunity to probe the elastic properties on the inner surface of the viral shell as well. In addition to the force-probe simulations described above, the tip-sphere was also placed in the water inside the capsid, and subsequently directed towards the inner surface of the shell. Thus, the tip-sphere was pushed along the same 19 vectors perpendicular to the tangent of each of the 19 grid points, only the sign of the vector, the tip-sphere was pushed along, was inverted compared to the simulations in which the tip-sphere was pushed against the same grid point on the outer surface.

When the tip-sphere was placed within the simulation system after equilibration of the protein and the water atoms, the box geometry of the rhombic dodecahedral box had to be considered (see section 3.1.3): When applying the GROMACS force-probe module to an atom or molecule of the simulation systems, the chosen pull-group (here: tip-sphere) had to be inside the simulation box for the rhombic dodecahedron AND the triclinic box as well and not in the periodic image. If the tip-sphere moved out of the box during the simulation, the force calculation resulted in an error and the simulation stopped. Therefore a subunit had to be chosen that was inside the simulation box and not in the periodic image (Fig. 3.2). Again it had to be ensured that the vector along which the tip-sphere was pushed was in the simulation box as well. Therefore, for SBMV, the grid points towards which the tip-sphere was directed, were on the surface of subunit (SU) 12 and on the surface of SU 35 for HRV 16.

During all FPMD simulations the center of mass (COM) motion of the viral shell was removed to fix the capsid position during indentation with the tip-sphere. Nevertheless, test-simulations were performed without COM motion of the capsid. It turned out that the results where identical because the force that was exerted by the tip-sphere on the viral shell was too small to push the complete capsid through the bulk water.

Before extensive force-probe simulations were carried out, the results of the CVS version were compared with results from GROMACS-3.3.1 to ensure that the CVS version was bug-free for our simulations. To rule out anecdotal events, all forceprobe simulations were performed at least twice. All force-probe simulations were carried out with probe-velocity of $0.01 \mathrm{~nm} / \mathrm{ps}$ where no other information is given. 


\subsection{Analysis}

Elastic constants from capsid indentation were calculated from the linear regime in the force-distance plot, using linear regression (see e.g. Fig. 4.12). The start and end points of the linear regimes were determined manually. Yielding forces were obtained from the maximum force in each force-distance plot.

To graphically represent the distribution of elastic constants and yielding forces on the surface of the viral shell and subunit, the elastic constants and yielding forces, respectively, of the 19 chosen grid points were used and the respective values interpolated at the position of all atoms of the subunit, using Gaussian functions. Accordingly, the elastic values $K_{j}^{\prime}$ for atom $j$ at position $\mathbf{x}_{j}$ were calculated as

$$
K_{j}^{\prime}=\frac{\sum_{i} K_{i} \cdot \exp \left(\frac{-\left(x_{i}-x_{j}\right)^{2}}{\sigma^{2}}\right)}{\sum_{i} \exp \left(\frac{-\left(x_{i}-x_{j}\right)^{2}}{\sigma^{2}}\right)},
$$

with a Gaussian width of $\sigma=0.8 \mathrm{~nm}$, chosen to optimally interpolate between the distances of the 19 grid points. Here $K_{i}$ is the elastic value of one of the 19 grid points.

To visualize the deformation of the viral shell proteins during indentation with the tip-sphere, the root mean square deviation (rmsd) and its evolution in time was calculated for each residue with respect to the initial structure of the complete capsid. Subsequently, the residues were color-coded in VMD [104] for their obtained rmsd values for various simulation time steps.

\section{Root mean square deviation}

The root mean square deviation (rmsd) is used as a measure of the average distance between a (protein) structure at time $t_{0}$, compared to the reference structure at time $t_{0}$ [105]. For a distribution of atoms at $t_{0}$ and $t_{1}$, the rmsd can be calculated as

$$
\operatorname{rmsd}\left(t_{0}, t_{1}\right)=\sqrt{\frac{1}{M} \sum_{i=1}^{N} m_{i}\left\|\mathbf{r}_{i}\left(t_{1}\right)-\mathbf{r}_{i}\left(t_{0}\right)\right\|^{2}}
$$

with $M=\sum_{i=1}^{N} m_{i}$ and $\mathbf{r}_{i}(t)$ the position of atom $i$ at time $t$. An rmsd value is expressed in length units, usually nanometer.

\section{Radius of gyration}

The radius of gyration $\left(R_{\text {gyrate }}\right)$ describes the radial distance from a given axis at which the mass of a body could be concentrated without altering the rotational inertia of the body about that axis. $R_{\text {gyrate }}$ is related to the root mean square distance of the atoms from the center of mass of the complete protein structure: 


$$
R_{g}=\sqrt{\frac{\sum_{i}\left\|\mathbf{r}_{i}\right\|^{2} m_{i}}{\sum_{i} m_{i}}}
$$

$m_{i}$ defines the mass of atom $i$ at position $\mathbf{r}_{i}$ with respect to the center of mass of the complete protein structure.

\section{Radial distribution function}

The radial distribution function ( $\mathrm{rdf}), g(r)$, describes the probability density of finding an atom $i$ at a certain distance away from another atom $j$ :

$$
g(r)=\frac{V}{4 \pi r^{2} N^{2}}\left\langle\sum_{i} \sum_{j \neq i} \delta\left(r-r_{i j}\right)\right\rangle .
$$

Here $N$ is the total number of particles at position $r$ in the system with volume $V$. Thus $g(r)$ provides a measure of local spatial ordering in a solid, fluid or gaseous material.

\subsection{Scaling behavior on parallel machines}

Most FPMD simulations were performed on 32 or 64 processors of an SGI Altix 4700 cluster of the Leibniz-Rechenzentrum in Munich. The scaling behavior for the simulation system of SBMV is given in table 3.1.

\begin{tabular}{lll}
\hline CPUs & time $[\mathrm{s}]$ & scaling \\
\hline 4 & 9.19 & $100 \%$ \\
16 & 2.45 & $94 \%$ \\
32 & 1.20 & $96 \%$ \\
64 & 0.62 & $92 \%$ \\
\hline
\end{tabular}

Table 3.1: Scaling behaviour of GROMACS-CVS on the SGI Altix 4700/HLRB2. The second column shows the real time elapsed for one integration time step.

As a comparison: 1 ns $(=500,000$ integration steps $)$ of simulation takes $\sim 7$ days on $32 \mathrm{CPUs}$ on the Altix SGI and 10 days on 32 processors on the $2 \mathrm{GH}$ Opteron clusters.

Several hundred MD and force-probe simulations were performed, totaling over 2,400,000 CPU hours of computing time for Southern Bean Mosaic Virus and Human Rhinovirus 16. 


\section{The mechanical properties of Southern Bean Mosaic Virus}

\subsection{Introduction to Southern Bean Mosaic Virus}

Southern Bean Mosaic Virus (SBMV) is a small plant virus which is common in the southern U.S., but it was also found in Marocco and France. Otherwise it is not widespread or an economic problem. Symptoms include crinkled leaves expressing a mild mosaic. The virus is beetle transmitted and seedborne at low efficiencies. SBMV consists of a coat protein and a positive single-stranded RNA inside, whereby two different strains exist: Cowpea strain and bean strain. In vitro studies showed that there are similarities in the overall mode of translation of the genomes of the two viruses [106]. Studies by Shepherd et al. exhibited that the bean virus cannot infect cowpea and vice versa [107]. Currently, only the x-ray structure of the coat protein of SBMV-cowpea strain is known [108].

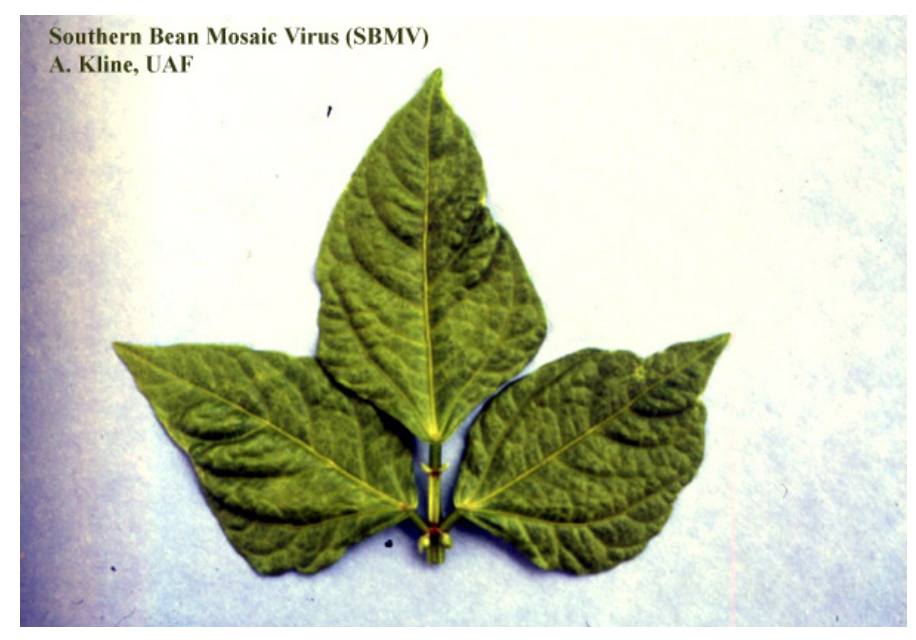

Figure 4.1: Bean leaves infected by Southern Bean Mosaic Virus. Taken from [109].

In this study, the viral shell of SBMV, cowpea strain [110] was chosen to investigate its mechanical properties from force-probe simulations, because its a prototypical viral capsid with icosahedral shell geometry. Furthermore, this virion has a small diameter $(\sim 32 \mathrm{~nm})$ compared to other plant and animal viruses, and the complete X-ray structure of the capsid has been solved at $2.9 \AA$ resolution [111] (PDB code: 4SBV). 
The geometrical configuration of the SBMV coat protein was first reported in 1960 [112]. The capsid exhibits a $T=3$ symmetry and is organized in 60 subunits with 3 almost identical proteins each. The subunit proteins are built by 260 amino acid residues with a relative molecular weight of 28,200 [113] folded into an eight-stranded antiparallel $\beta$-barrel. In total, 180 proteins and RNA sum up to a molecular weight of $6.6 \cdot 10^{6}$ [114], $21 \%$ in weight accounts for the genetic material. The diameter varies between $285 \AA$ at the quasi-3-fold axes and $328 \AA$ along the 5-fold axes (Fig. 3.4), the average shell thickness is $35 \AA$ [108].

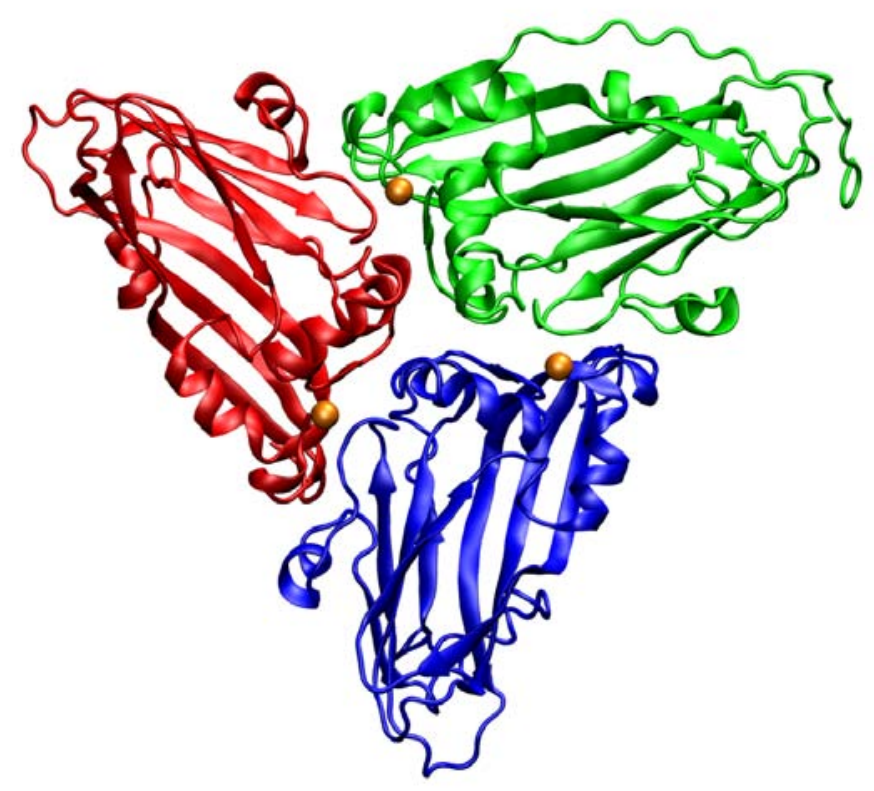

Figure 4.2: Subunit of Southern Bean Mosaic Virus. Protein A: blue, Protein B: red, Protein C: green. The three calcium ions are shown as orange spheres.

The subunits form equilateral triangles with side length of $\sim 7.5 \mathrm{~nm}$, spanned by protein $\mathrm{A}, \mathrm{B}$ and $\mathrm{C}$ with identical amino acid sequences. The refined crystal structure comprises residues 62 to 260 for protein A and B and residues 39 to 260 for residue C. The missing residues are hidden inside the capsid and interloop with the RNA. Although chemically identical, subunits related by quasi-3-fold axes at the intersection point of protein A, B, C are structurally non-equivalent, since they are involved in different types of subunit contacts [115]. Proteins A make pentameric clusters (pentamers) around icosahedral 5-fold symmetry axes, while protein $\mathrm{B}$ and $\mathrm{C}$ form hexameric clusters (hexamers) around icosahedral 3-fold symmetry axes (Fig. 3.4). The angle between the A proteins around the pentamers is $140^{\circ}$ and alternates between $140^{\circ}$ and $180^{\circ}$ for protein $\mathrm{B}$ and $\mathrm{C}$ around the hexamers.

Every subunit contains three $\mathrm{Ca}^{2+}$-ions at the interfaces of protein $\mathrm{A}, \mathrm{B}$ and $\mathrm{C}$ [116] (Fig. 4.2). The $\mathrm{Ca}^{2+}$ binding sites are located close to the external viral surface, about 14A from the quasi-3-fold axes (subunit center). SBMV, as well as Tomato Bushy Stunt Virus, requires metal ions for assembly and stabilization of the viral 
shell. The function of calcium ions has been extensively studied for SBMV [117119]. It turned out that the absence of $\mathrm{Ca}^{2+}$ and a $\mathrm{pH}$ of 7.5 cause a swelling of the virion by about $7 \%$ in volume [60]. Brisco et al. reported a swelling to $44.0 \mathrm{~nm}$ virion diameter at $\mathrm{pH} 8.25$ within 3 minutes [120].

Until now it is not clear if and how the swelling of the capsid affects the mechanical properties of the viral shell and thus advances RNA release. Only the fact that the capsid has to swell to release RNA is accepted but why and how the swelling proceeds is a matter of debate: SBMV particles may behave in vivo like those of Turnip Yellow Mosaic Virus, which releases all its RNA within minutes of contact with leaves [121] or of those of Belladonna Mottle Virus, which releases its RNA immediately when the $\mathrm{pH}$ is raised above 7 in vitro [122]. Hence, the full-length RNA of both these viruses is released so rapidly that, alternatively, SBMV may behave like Tobacco Mosaic Virus [123], in which the RNA is uncoated sequentially during translation [124] (Step 2 and partially step 3 in Fig. 1.1). Shields et al. concluded that SBMV particles disassemble only after its RNA has initiated translation [23].

One possible pathway for RNA release was suggested by Silva et al. [125]. They found that the coat protein of SBMV, as well as other icosahedral viruses like rhinovirus, forms a $30 \AA$ long ion channel along the 5-fold symmetry axes through the center of the pentamers. The biological role of these channels could not be determined by now. They might be just selective leak channels providing cations to ensure electrical neutrality of the RNA and then stabilize the inner viral media. Another possibility is that the channels are related to inducing, in vivo, destabilization of the capsid by supporting a swelling process of the capsid, and subsequently act as a port and release the RNA into the cell. Alternatively, parts of the viral shell might become incorporated into the cell membrane, changing its permeability towards conditions favoring viral functions [125]. Nevertheless, it should be mentioned that the channels would be adequate to transport $\mathrm{Ca}^{2+}$ ions and water as well, but there is also a sharp energy barrier at the gate of $\Delta E \approx 300 \mathrm{kcal} / \mathrm{mol}$ for water and $\Delta E \approx 600 \mathrm{kcal} / \mathrm{mol}$ for calcium ions in SBMV. No other region of the protein shell exhibited a gate for possible water or ion permeation.

In the following, the mechanical properties of SBMV with and without $\mathrm{Ca}^{2+}$ ions are studied with force-probe molecular dynamics studies. From the differing mechanical properties obtained on the inner and outer surface of SBMV capsid with and without calcium ions in the structure, a possible port for RNA release is suggested. 


\subsection{Simulation details}

\subsubsection{Molecular dynamics simulations}

The x-ray structure of SBMV [81] at $2.9 \AA$ resolution was taken from the PDB (code: 4sbv) and VIPER data bank. After addition of hydrogen atoms to the protein, solvation in TIP4P water and the addition of sodium and chloride ions, the simulation box contained 564,000 protein atoms, including $180 \mathrm{Ca}^{2+}$ ions, $5500 \mathrm{Na}^{+} / \mathrm{Cl}^{-}$ions and approx. 1,000,000 water molecules, which sum up to more than 4.5 million particles (see also chapter 3.1.4).

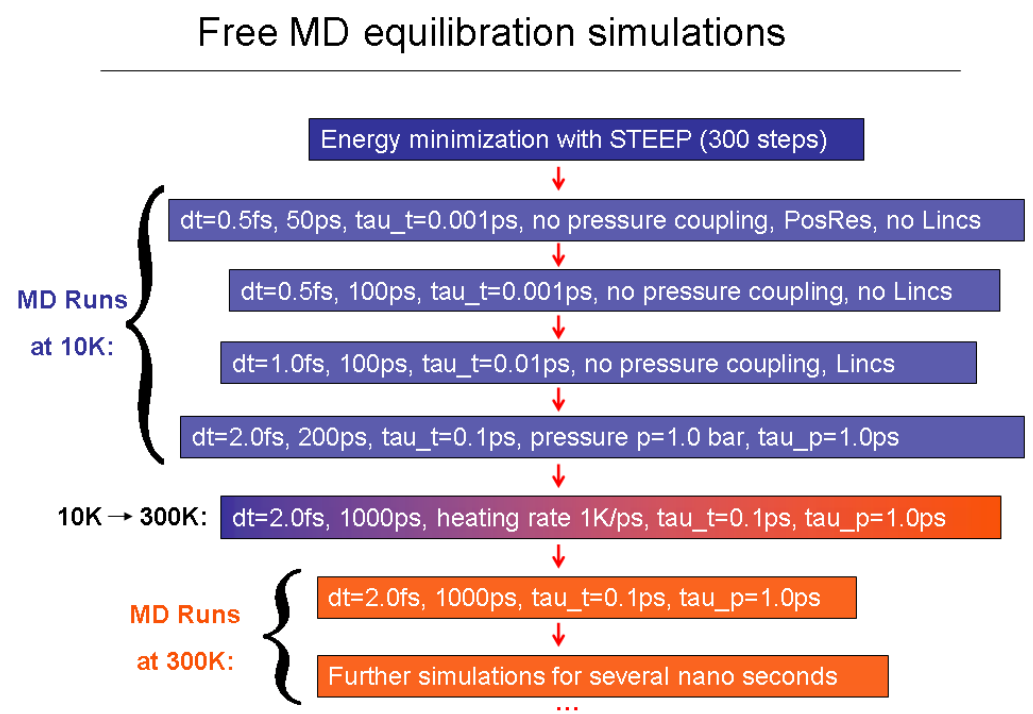

Figure 4.3: Protocol for energy minimization and equilibration simulations for SBMV solvated in water.

Fig. 4.3 depicts the procedure of energy minimization and subsequent free equilibration simulations of the simulation system, which was first energy minimized with a 300 steps steepest decent algorithm. Subsequently, the system was equilibrated by $\mathrm{MD}$ runs at $10 \mathrm{~K}$ and $300 \mathrm{~K}$ as follows. At the beginning, the system was coupled to a heat bath with $\tau_{T}=0.001 \mathrm{ps}$, with no pressure coupling applied. A $50 \mathrm{ps} \mathrm{MD}$ simulation with integration time steps of $0.5 \mathrm{fs}$ and harmonically constrained heavy protein atoms with a force constant of $k=1000 \mathrm{~kJ} \mathrm{~mol}^{-1}$ was performed. For the subsequent 100 ps simulation, the constraints were released, and further 100 ps were performed with integration steps of $\Delta t=1.0 \mathrm{fs}$ with all bonds constrained using the LINCS algorithm [91] and a weaker temperature coupling of $\tau_{T}=0.01$ ps. Integration steps of $2.0 \mathrm{fs}$ were used for further $200 \mathrm{ps}$ with $\tau_{T}=0.1 \mathrm{ps}$ and a pressure coupling at 1.0 bar, $\tau_{p}=1.0 \mathrm{ps}$. Finally, the simulation system was heated up to $300 \mathrm{~K}$ with a heating rate of $1 \mathrm{~K} / \mathrm{ps}$ and subsequently equilibrated for $19.5 \mathrm{~ns}$, in total, to ensure proper relaxation of the protein and water molecules. 
To set up a simulation system in which no calcium ions were included in the capsid structure, the $180 \mathrm{Ca}^{2+}$ ions were removed from the simulation system after $3 \mathrm{~ns}$ of equilibration as described above, because an x-ray structure without $\mathrm{Ca}^{2+}$ is not available. The salt concentration was adapted to neutralize the system. Subsequently, the system was energy minimized with 300 steepest decent steps and further equilibrated at $300 \mathrm{~K}$ for $32 \mathrm{~ns}$ with free molecular dynamics simulations with the same simulation parameters as used for the simulation system with $\mathrm{Ca}^{2+}$.

\subsubsection{Force-probe molecular dynamics simulations}

Force-probe molecular dynamics simulations were performed towards 19 different grid points of subunit (SU) 12 of Southern Bean Mosaic Virus (Fig. 4.4) with a probe-velocity of $0.01 \mathrm{~nm} / \mathrm{ps}$.

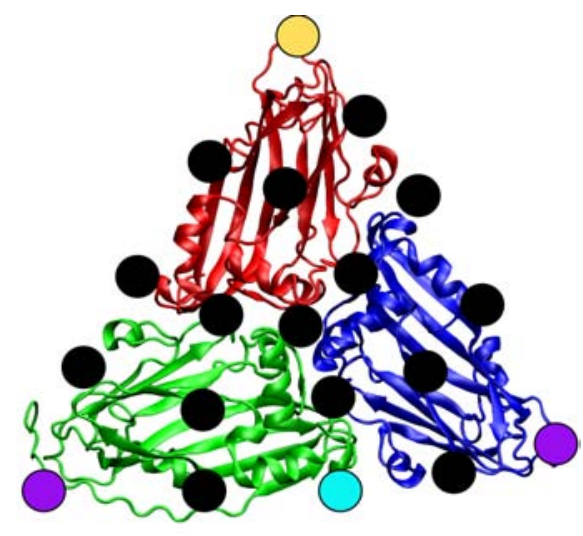

Figure 4.4: Subunit 12 of SBMV with grid points (dots) to which the tip-sphere was directed. The yellow dot depicts the grid point at the 5-fold symmetry axis, the purple dots are located at the 3 -fold symmetry axes, and the cyan dot at the 2-fold axis.

Four simulation series were performed. In the first series (1), the tip-sphere was positioned within the water shell outside the viral shell and pushed against the 19 grid points on the outer surface of SBMV including $\mathrm{Ca}^{2+}$-ions. (2) The tip-sphere was placed inside the virus and pushed against the same grid points on the inner surface of SBMV with calcium. (3) Identical simulations as in (1), but for the simulation system which did not include $\mathrm{Ca}^{2+}$ in the capsid structure. (4) Simulation as described in (2), but without $\mathrm{Ca}^{2+}$.

To rule out anecdotal events, all force-probe simulations were performed at least twice. The first run of FPMD simulations including calcium ions was started from structures extracted after 12 ns of the equilibration run, the second run from structures taken after $13 \mathrm{~ns}$. Without $\mathrm{Ca}^{2+}$ ions, the first run was performed after an equilibration of the structure for $14 \mathrm{~ns}$, the second run after $15 \mathrm{~ns}$. A total of 152 
indentation simulations for the four simulation series was carried out, each comprising 38 simulations.

Additional force-probe simulations were performed from structures extracted after $19.5 \mathrm{~ns}$ of equilibration for the capsid with calcium ions and $32 \mathrm{~ns}$ without calcium to investigate the effect of equilibration and convergence of the protein structure on the mechanical properties. 


\subsection{Results}

\subsubsection{Energy minimization and equilibration of the simulation system}

\section{Viral shell with calcium ions}

The simulation system including the calcium ions was first equilibrated for $19.5 \mathrm{~ns}$, in total. As a start, the complete simulation system was energy minimized with 300 steepest descent steps. With respect to the maximum forces acting on the atoms after steepest descent which were of the order of $10^{4} \frac{\mathrm{kJ}}{\mathrm{mol}}$, the system was subsequently equilibrated very smoothly with MD simulations. For the simulation system including calcium ions, the different equilibration steps (Fig. 4.3) are also represented in the rmsd curve of the viral shell heavy atoms compared to the protein structure extracted after 300 steepest descent steps (Fig. 4.5 and 4.6).

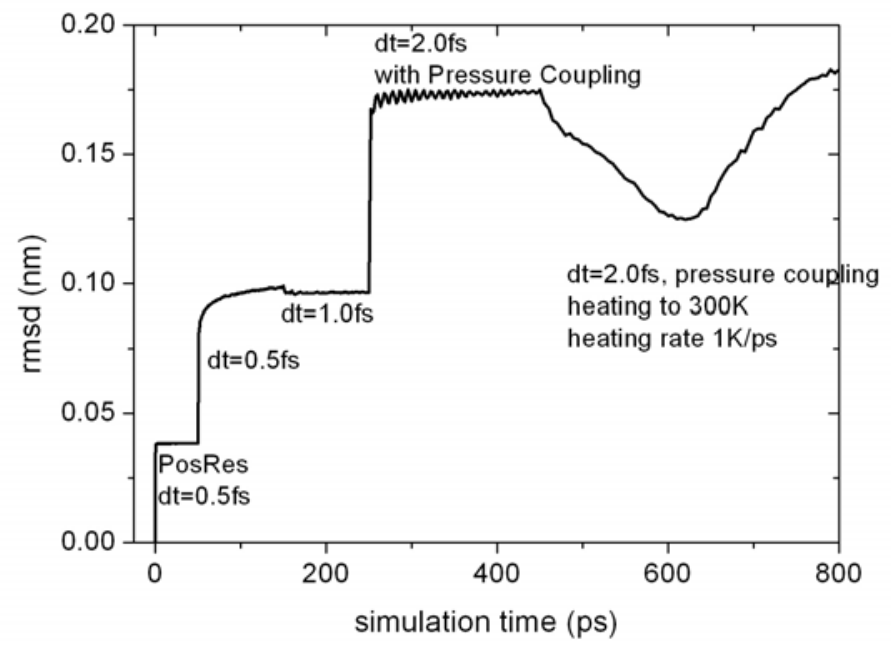

Figure 4.5: Rmsd of the simulation system fitted on the protein structure obtained after steepest descent steps during the first $800 \mathrm{ps}$ of equilibration simulations.

As can be seen in Fig. 4.5, the protein atoms barely moved during the first $50 \mathrm{ps,}$ because of position restraints on the heavy atoms. After their release at $50 \mathrm{ps}$, the rmsd increased and leveled off at $0.1 \mathrm{~nm}$ due to the low system temperature of $10 \mathrm{~K}$ and the hampered atom and bond vibrations. At $250 \mathrm{ps}$, pressure coupling was employed, which caused a compression of the viral shell seen as a dip in the radius of gyration (Fig. 4.7), and an increase of the rmsd to $0.17 \mathrm{~nm}$. When the water molecules were put into the simulation box with the GROMACS tool genbox, the number of solvent molecules for a constant box size was calculated for a system temperature of $300 \mathrm{~K}$. Therefore, the number of water molecules was less for $300 \mathrm{~K}$ 


\subsection{Results}

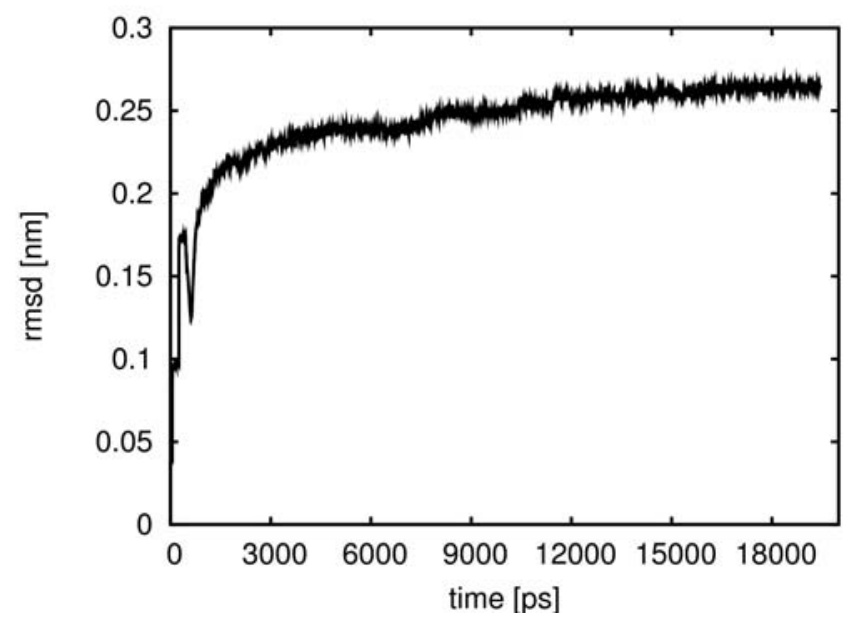

Figure 4.6: Rmsd of the simulation system fitted on the starting structure after steepest descent steps as a function of time for the complete $19.5 \mathrm{~ns}$ of equilibration.

as it would be for $10 \mathrm{~K}$, and the pressure coupling rescaled the system size to a smaller volume to set the pressure to 1 bar at $10 \mathrm{~K}$.

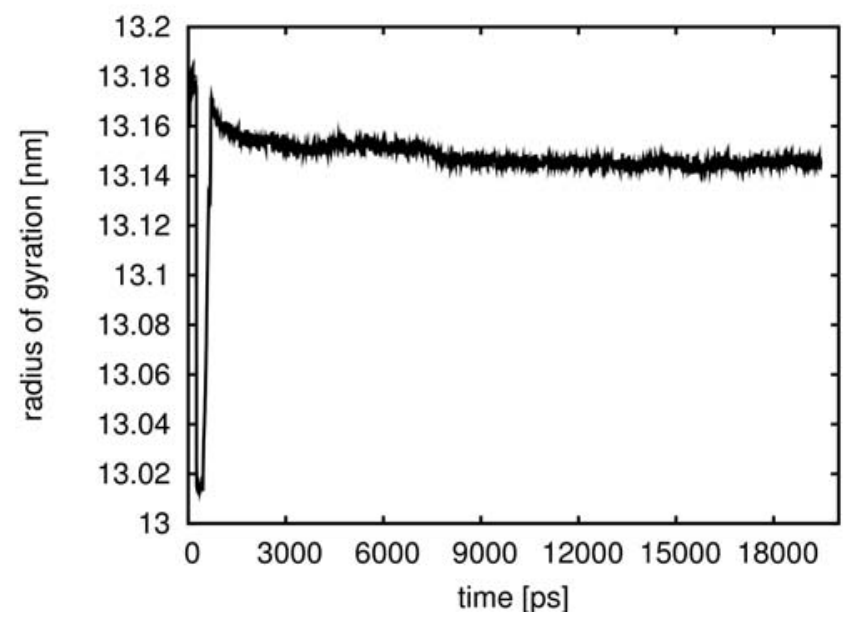

Figure 4.7: Time-evolution of the radius of gyration of the viral shell during equilibration of the simulation system.

During heating, the capsid radius relaxed and the protein atoms approached their original positions, causing a dip in the rmsd plot at $450 \mathrm{ps}$, followed by a slight further expansion. Here the radius of gyration increased until it reached its initial value at $131.7 \AA$ at $600 \mathrm{ps}$. The rmsd increased from $1.96 \AA$ at $1.0 \mathrm{~ns}$ to $2.50 \AA$ at $10 \mathrm{~ns}$ and subsequently remained stable at $2.62 \AA$ after $13 \mathrm{~ns}$ until $19.5 \mathrm{~ns}$, the end of the equilibration phase. 
The radius of gyration leveled off at $131.5 \pm 0.5 \AA$ after $8 \mathrm{~ns}$, only $0.2 \AA$ smaller than the value of the $\mathrm{x}$-ray structure. This small difference in the radius of gyration is mainly caused by flexible loops at the inner surface of the protein which changed their positions during the equilibration runs.

To investigate the distribution of the protein atoms that build up to the viral capsid, the radial distribution function (rdf) of the protein with respect to the center of mass of the capsid was calculated during the last $1 \mathrm{~ns}$ of equilibration. Most atoms were located $13.2 \mathrm{~nm}$ away from the viral center, which reflects the radius of gyration. The minimum distance to the center was $\sim 10.0 \mathrm{~nm}$ (flexible loops along the quasi 3 -fold symmetry axes) and the maximum distance $\sim 16.8 \mathrm{~nm}$ (flexible loops on the outer pentamer surface along the 5 -fold symmetry axes).

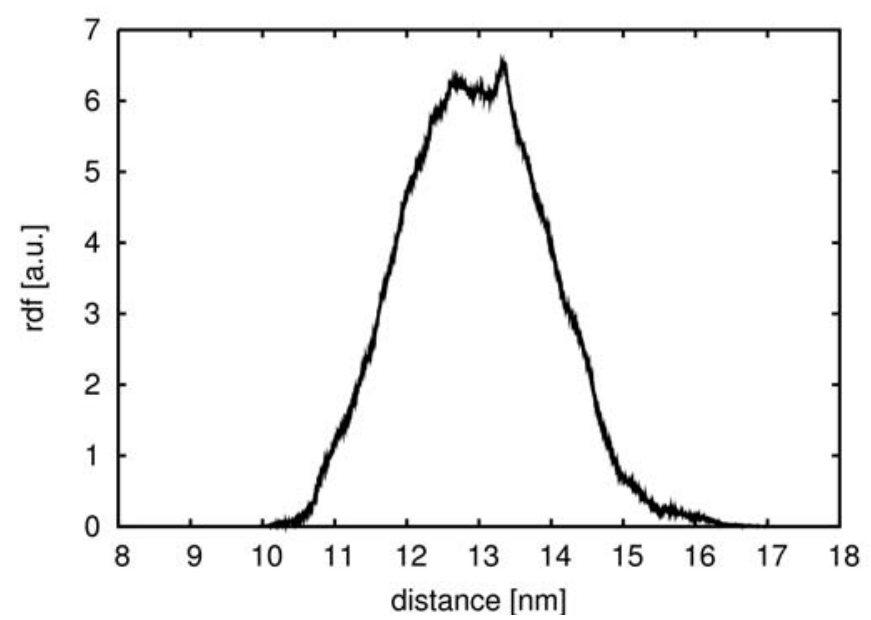

Figure 4.8: Radial distribution function (rdf) of the viral shell with respect to the center of mass of the capsid. The minimum distance of the protein atoms to the capsid center is $\sim 10.0 \mathrm{~nm}$, the maximum distance $\sim 16.8 \mathrm{~nm}$.

Although a complete equilibration of the protein atoms could not be guaranteed after $19.5 \mathrm{~ns}$ of equilibration because the rmsd could still increase for longer simulation times, the system was considered sufficiently equilibrated after $13 \mathrm{~ns}$ for the mechanical properties studied here and force-probe simulations were performed from structures extracted from simulations between $12.0 \mathrm{~ns}$ and $13.0 \mathrm{~ns}$ of free MD equilibration runs. As a test for sufficient relaxation, several additional force-probe simulations were performed after $19.5 \mathrm{~ns}$ of equilibration. Comparison of the FP simulations started from structures after $13 \mathrm{~ns}$ and $19.5 \mathrm{~ns}$ of equilibration revealed no significant differences in the mechanical properties. The values for the elastic constants and yielding forces obtained after $19.5 \mathrm{~ns}$ were within the errors of the values obtained after $13 \mathrm{~ns}$ of equilibration (see below).

Finally it should be mentioned that the bulk water was checked for cavities during 


\subsection{Results}

equilibration to ensure that enough water molecules were present in the simulation box, especially inside the viral shell. The GROMACS tool genbox was used to fill additional water molecules in possible cavities. It turned out that no extra water molecules fitted in the simulation box. Next, the density of the simulation system was determined. A biomolecular system with solvated proteins usually exhibits a density of approx. 900-1000 g/l [126]. For the underlying simulation box with viral shell, water and ions, a density of $1050 \mathrm{~g} / \mathrm{l}$ was obtained at a pressure of $1.0 \mathrm{bar}$, which leads to the conclusion that it is very unlikely to find cavities in the bulk water after equilibration. As a last test, a cube of equilibrated water inside the capsid was cut out of the simulation system, placed into a new simulation box and equilibrated for $1 \mathrm{~ns}$. The monitored pressure was approx. 1 bar, and we conclude that no pressure gradient inside and outside the capsid occurred when the pressure coupling was applied.

\section{Viral shell without calcium ions}

The second simulation system, consisting of the solvated shell of SBMV without $\mathrm{Ca}^{2+}$-ions in the atomic structure of the capsid, was equilibrated for $32 \mathrm{~ns}$. When calcium was removed from the system, the rmsd increased from $1.70 \AA$ at $1.0 \mathrm{~ns}$ to $2.28 \AA$ at $10 \mathrm{~ns}, 2.52 \AA$ after $15 \mathrm{~ns}$ and leveled off at $2.71 \AA$ after $25.5 \mathrm{~ns}$ until the end of the equilibration at $32 \mathrm{~ns}$. Although the rmsd did not fully converge after $15 \mathrm{~ns}$, we used that $15 \mathrm{~ns}$ equilibrated simulation system as the initial structure for our 156 force-probe molecular dynamics simulations.

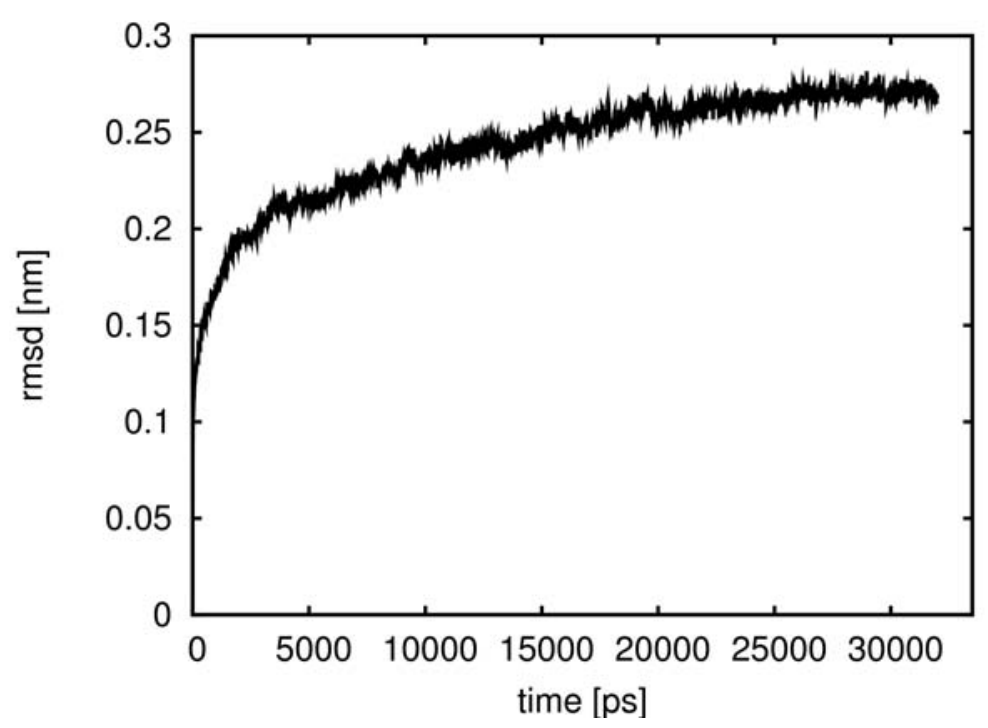

Figure 4.9: Rmsd of viral shell heavy atoms during equilibration, fitted on the starting structure after calcium removal. 
Additional force-probe simulations were performed using the structure extracted after $32 \mathrm{~ns}$ of equilibration. The obtained values for the mechanical properties were within the errorbars of the results found from simulations after a 15 ns equilibration phase. We therefore consider the simulation system sufficiently relaxed after $15 \mathrm{~ns}$ of equilibration to study the capsid's elastic behavior.

In contrast to the swelling of the capsid seen in experiments after $\mathrm{Ca}^{2+}$ removal, the radius of gyration decreased slightly by $0.2 \AA$ and a swelling could not be observed on time scales of the simulation.

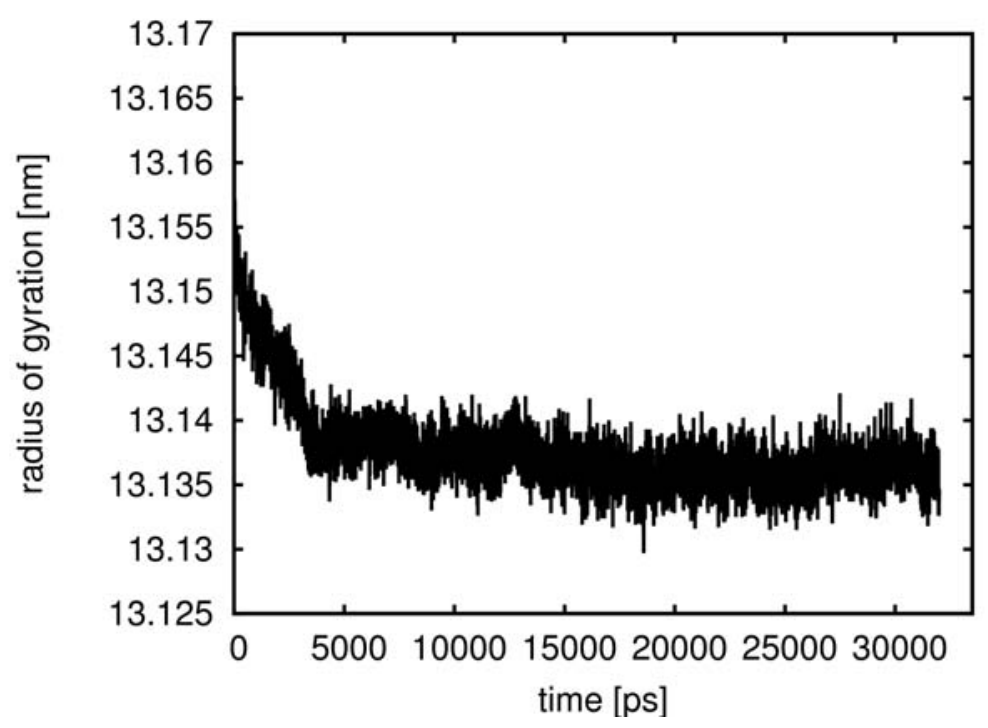

Figure 4.10: Radius of gyration of the capsid without calcium ions during the equilibration phase.

An rmsd of $2.3 \pm 0.3 \AA$ between the equilibrated structures of SBMV with and without calcium was found, whereas the structure of the $\beta$-barrel remained stable for both structures and the differences in the rmsd arose from the flexibility of the loops (Fig. 4.11). 


\subsection{Results}

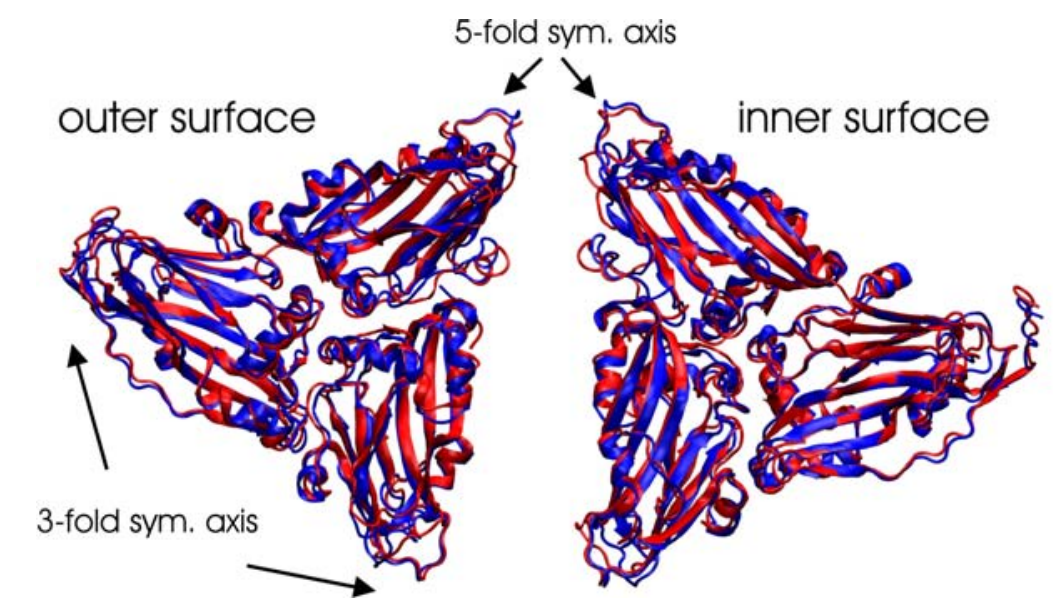

Figure 4.11: Cartoon representation of subunit 12 on the inner and outer surface. Both structure with calcium ions (red) and without ions (blue) were extracted after the respective equilibration simulations. 


\subsubsection{Deformation of the capsid}

In order to study the spatial distribution of elastic properties on the inner and outer shell surface of Southern Bean Mosaic Virus with and without calcium ions, four force-probe simulation series were performed. Each series contained two FP simulations in which the tip-sphere was directed towards every grid point, totaling 152 simulations and $\sim 110 \mathrm{~ns}$ in length. At the beginning of each simulation, the tip-sphere was positioned in the water, close to the viral surface and subsequently pushed through the water against the viral shell. During the force-probe simulation, the force acting on the tip-sphere was monitored; Fig. 4.12 shows a typical forcedistance and force-time curve. Here, the tip-sphere was positioned close to the outer surface of protein A, subunit (SU) 12 and pushed with a probe-velocity of $0.01 \mathrm{~nm} / \mathrm{ps}$ against the $\beta$-sheet region of the protein.

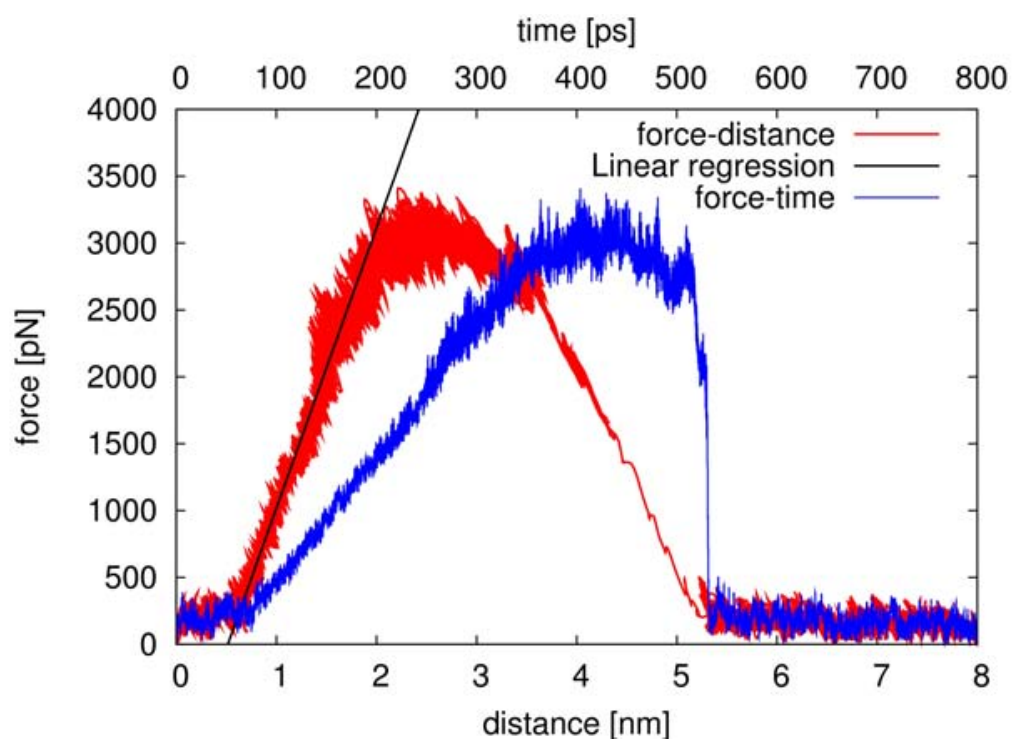

Figure 4.12: Force-distance plot (red) and force-time plot (blue) for pushing the tip-sphere with a velocity of $0.01 \mathrm{~nm} / \mathrm{ps}$ against the $\beta$-sheet of protein A, subunit 12. The black line marks the linear regime where elastic deformation takes place.

At the beginning of the simulation, the tip-sphere moved through the water for $70 \mathrm{ps}$ before it touched the capsid, at which point the force increased. From the slope of the linear regime in the force-distance curve the elastic constant of the capsid at the respective position was obtained. After approx. $250 \mathrm{ps}$, the force bent over to a nonlinear behavior and finally leveled off at $420 \mathrm{ps}$ at a yielding force of $F_{\max }=3205 \mathrm{pN}$. Subsequently, the tip-sphere was pushed inside the capsid and left the shell, resulting in a rapid force decline. A detailed description of the forcedistance and force-time curves is given in chapter 5 . 


\subsubsection{Elastic constants}

From the four force-probe simulation series, each containing 38 simulations in which the tip-sphere was pushed against the 19 evenly distributed grid points on SU 12 of SBMV with and without calcium ions, a heterogeneous distribution of elastic constants was obtained. The values varied between $1.65 \mathrm{~N} / \mathrm{m}$ and $4.33 \mathrm{~N} / \mathrm{m}$ (Fig. $4.14-4.17)$.

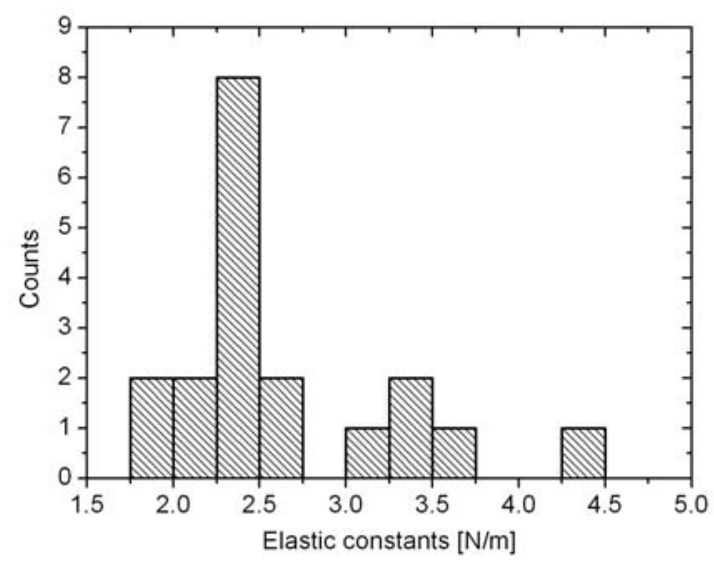

Figure 4.13: Histogram of the elastic constants from FP simulations in which the tip-sphere was pushed towards the 19 grid points on the outer surface of SU 12 with calcium ions in the capsid structure.

\section{Capsid with calcium, probed from outside}

When the tip-sphere was pushed from outside against the outer surface of SBMV with $\mathrm{Ca}^{2+}$ ions, the largest elastic constant $(4.33 \mathrm{~N} / \mathrm{m})$ was found at the 5 -fold symmetry axis (center of pentamer), followed by the two 3-fold symmetry axes (center of hexamers, $3.42 \mathrm{~N} / \mathrm{m}$ and $3.53 \mathrm{~N} / \mathrm{m}$, respectively, Fig. 4.14). With regard to the question whether pentamers or hexamers are more stable, the results show a significantly higher stiffness for the pentamers.

The weakest point was found at the center of the subunit (quasi-3-fold axis) with an elastic constant of $1.82 \mathrm{~N} / \mathrm{m}$, followed by the elastic constant along the 2-fold symmetry axis $(1.89 \mathrm{~N} / \mathrm{m})$. Interestingly, the elastic response for pushing the tipsphere towards the interface of SU 12 and 11, as well as the interface of SU 12 and 28 was consistently larger at the grid points where the A-proteins meet $(2.46 \mathrm{~N} / \mathrm{m}$ and $2.61 \mathrm{~N} / \mathrm{m})$ than between $\mathrm{B}$ and $\mathrm{C}$ proteins $(2.17 \mathrm{~N} / \mathrm{m}$ and $2.28 \mathrm{~N} / \mathrm{m}$, respectively). Next, we focused on the force-probe simulations in which the tip-sphere was pushed towards the three proteins inside SU 12. All resulting elastic constants were found to be in the range of $2.29-2.45 \mathrm{~N} / \mathrm{m}$, also at the interfaces of the subunit proteins $\mathrm{A}, \mathrm{B}$ and $\mathrm{C}$ where the calcium ions were located. The $\beta$-sheet regions turned out to be particularly stiff compared to those containing $\alpha$-helices and loops. 
a)

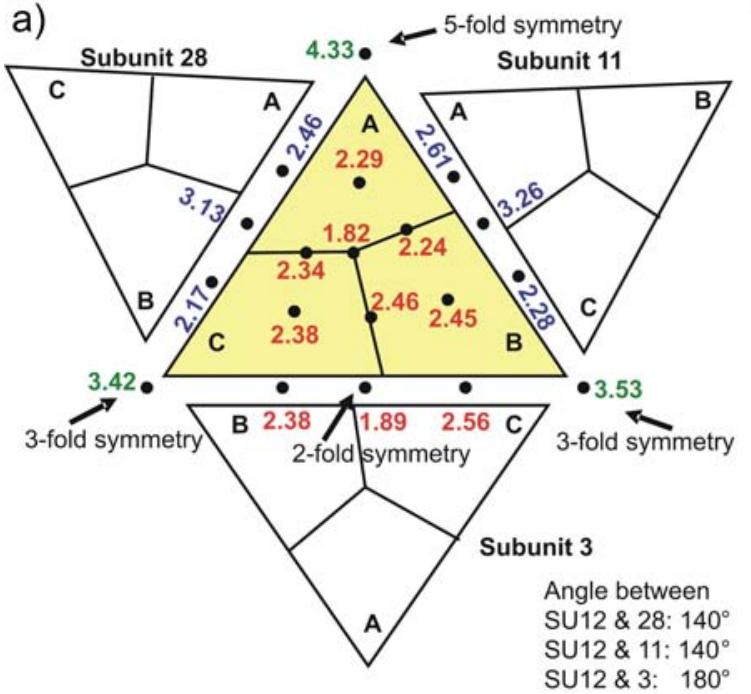

b)

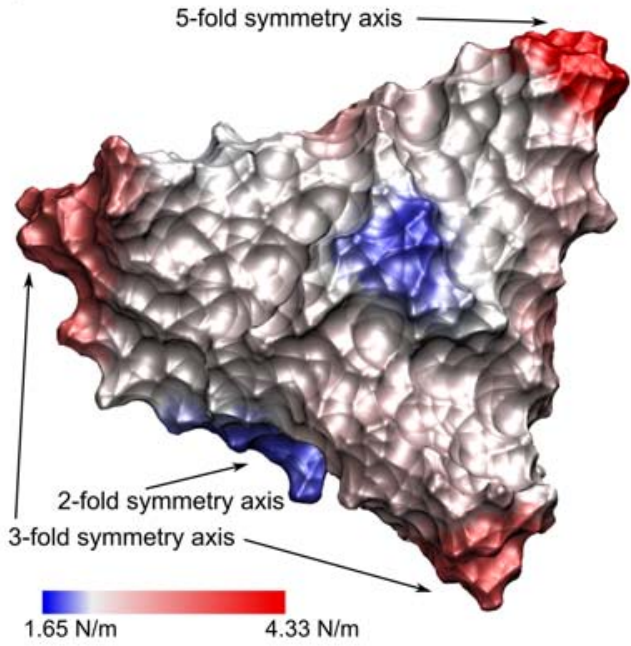

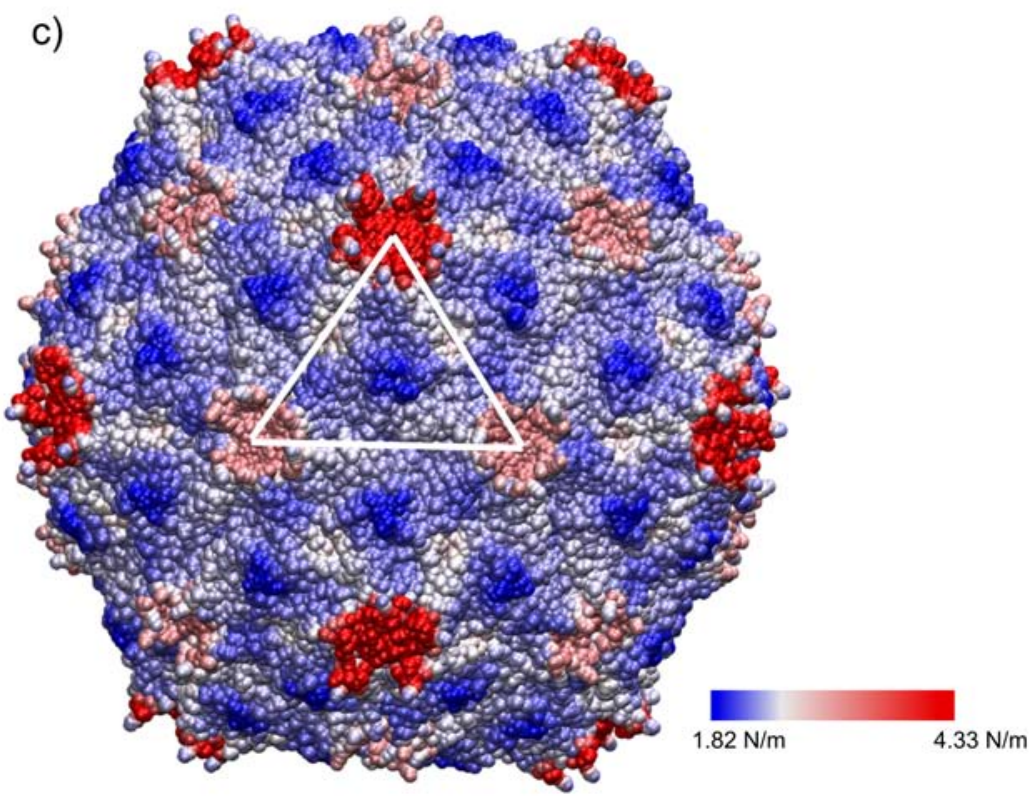

Figure 4.14: Elastic constants of the capsid with calcium, probed from outside. a) Sketch of SU 12 (yellow) and the adjacent subunits (white). The black dots mark the grid points at which the elastic properties have been determined. The obtained elastic constants (in $\mathrm{N} / \mathrm{m}$ ) are shown in red, green, blue; the different colors of the numbers denote different push-vector directions perpendicular to the viral surface. b) Colorcoded distribution of elastic constants on subunit 12, obtained from values shown in a) (soft: blue, stiff: red). The smallest elastic constant value depicted in the color-bar $(1.65 \mathrm{~N} / \mathrm{m})$ was taken from force-probe simulation results marked in Fig. 4.14. c) Capsid of SBMV color-coded for the elastic constants from values shown in a). One subunit is marked by the white triangle. 


\subsection{Results}

\section{Capsid with calcium, probed from inside}

When the tip-sphere was pushed from the capsid inside against the inner surface of SBMV with $\mathrm{Ca}^{2+}$ ions, the distribution of elastic constants changed, whereas inside SU 12 the values of the elastic constants were very similar to the value obtained on the outer surface within a deviation of $0.17 \mathrm{~N} / \mathrm{m}$ (Fig. 4.15). The subunit center was also the weakest point of the subunit $(1.65 \mathrm{~N} / \mathrm{m})$. The elastic constants at the interfaces of the subunits, where A-A proteins and B-C meet, were reduced and no significant differences in the values for A-A and B-C protein intersections were seen. Here, the 5-fold symmetry axis lost its rigidity and an elastic constant of $2.01 \mathrm{~N} / \mathrm{m}$ was found, $2.32 \mathrm{~N} / \mathrm{m}$ weaker as if the tip-sphere was pushed along the 5 fold symmetry axis from outside the capsid. Also the 3-fold symmetry axes became weaker, and the elastic constants dropped to $2.45 \mathrm{~N} / \mathrm{m}$ and $2.31 \mathrm{~N} / \mathrm{m}$, respectively. The only reinforcement was seen along the 2-fold symmetry axis; the elastic constant rose to $2.87 \mathrm{~N} / \mathrm{m}$, compared to the $1.89 \mathrm{~N} / \mathrm{m}$ on the outer surface. Therefore the grid point at the 2-fold symmetry axis exhibited the highest stiffness of the complete subunit and symmetry axes on the inner surface of the shell.
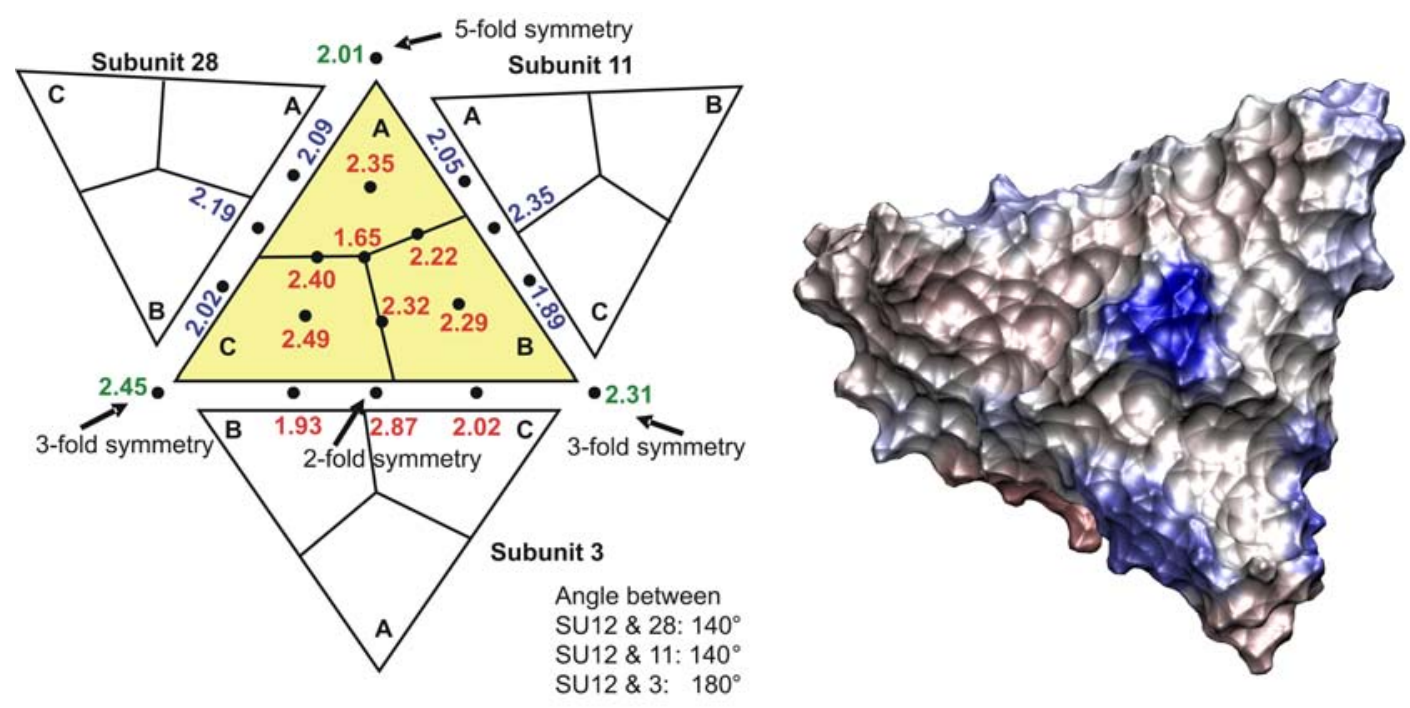

Figure 4.15: Elastic constants of the capsid with calcium, probed from inside. Obtained elastic constants (in $\mathrm{N} / \mathrm{m}$ ) and color-bar shown as in Fig. 4.14 .

\section{Capsid without calcium, probed from outside}

Next, the elastic constants of the capsid of SBMV were determined after removal of the calcium ions from the viral structure (Fig. 4.16). When the outer surface of the capsid was probes with the tip-sphere, we found the largest elastic constants at the pentamer center $(3.56 \mathrm{~N} / \mathrm{m})$ and the hexamer centers $(3.34 \mathrm{~N} / \mathrm{m}$ and $3.27 \mathrm{~N} / \mathrm{m}$, 
respectively), as already seen for the same capsid positions with $\mathrm{Ca}^{2+}$ ions in the structure. The elastic constants obtained at the grid points between the subunits where A-A proteins meet $(2.54 \mathrm{~N} / \mathrm{m}$ and $2.56 \mathrm{~N} / \mathrm{m}$, respectively), were also slightly larger than between B-C proteins $(2.28 \mathrm{~N} / \mathrm{m}$ and $2.40 \mathrm{~N} / \mathrm{m}$, respectively). In contrast to the simulation system including calcium, the elastic constants found for the subunit center $(2.23 \mathrm{~N} / \mathrm{m})$ and along the 2 -fold symmetry axis $(2.64 \mathrm{~N} / \mathrm{m})$ were increased and not the softest positions of the subunit anymore, as seen in the respective simulation with calcium. The smallest values were now obtained inside SU 12 at the interfaces of protein $\mathrm{A}, \mathrm{B}$ and $\mathrm{C}(2.10 \mathrm{~N} / \mathrm{m}, 2.10 \mathrm{~N} / \mathrm{m}$ and $2.07 \mathrm{~N} / \mathrm{m}$, respectively), where the $\mathrm{Ca}^{2+}$ ions were located before removal. The centers of the three proteins were strengthened, and the elastic constants increased by approx. $0.6 \mathrm{~N} / \mathrm{m}$ to $2.79 \mathrm{~N} / \mathrm{m}$ (protein A), $2.89 \mathrm{~N} / \mathrm{m}$ (protein B) and $2.31 \mathrm{~N} / \mathrm{m}$ (protein C).
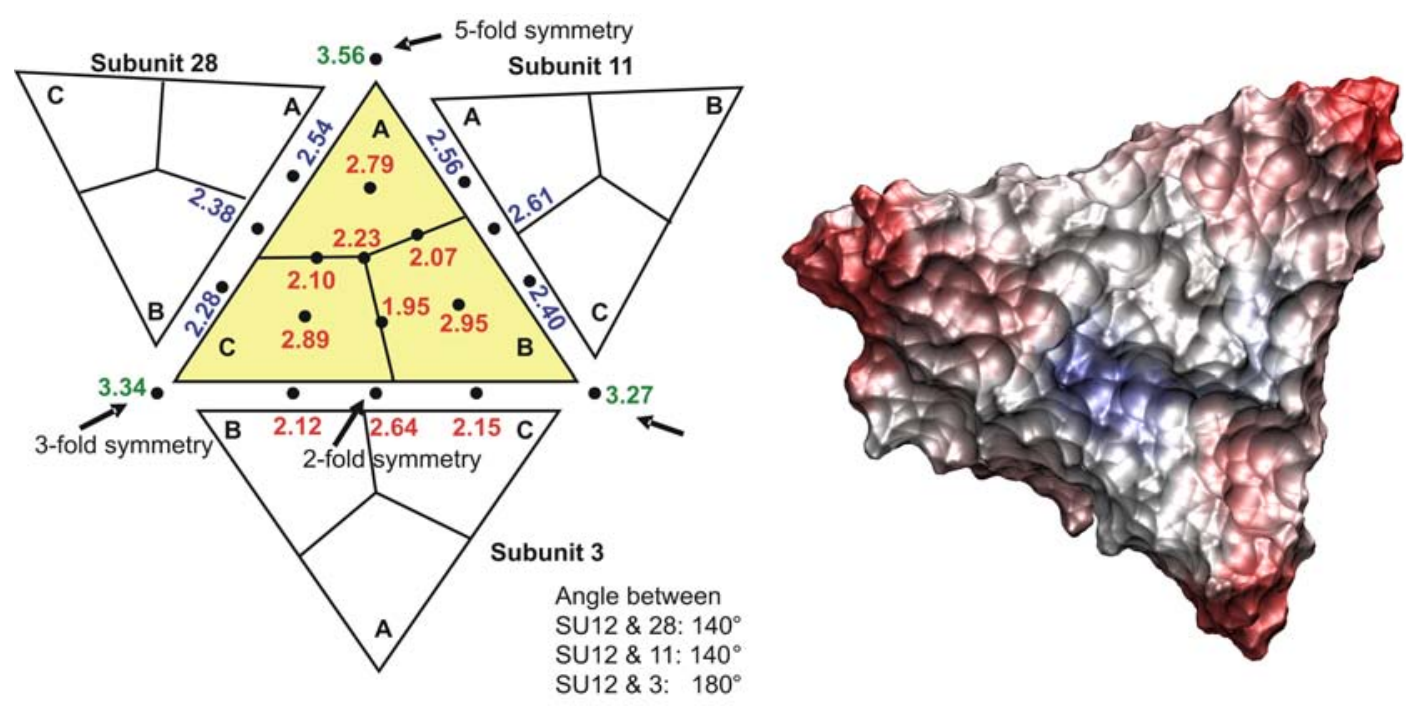

Figure 4.16: Elastic constants of the capsid without calcium, probed from outside. Obtained elastic constants (in $\mathrm{N} / \mathrm{m}$ ) and color-bar shown as in Fig. 4.14.

\section{Capsid without calcium, probed from inside}

Finally, the tip-sphere was pushed against the 19 grid points on the inner shell of SBMV without calcium in the structure (Fig. 4.17). Similar to the simulations in which the tip-sphere was pushed from outside against the capsid with $\mathrm{Ca}^{2+}$, we found the largest elastic constant at the pentamer center $(3.31 \mathrm{~N} / \mathrm{m})$, followed by the hexamer centers $(2.65 \mathrm{~N} / \mathrm{m}$ and $2.51 \mathrm{~N} / \mathrm{m}$, respectively), whereas for this simulation series without $\mathrm{Ca}^{2+}$, the respective values were much smaller.

The subunit exhibited the softest point at its center $(1.88 \mathrm{~N} / \mathrm{m})$. The elastic constants inside SU 12 were comparable to that found before, when pushing the tipsphere from outside against the capsid without calcium ions. The value at the 2 -fold 


\subsection{Results}

symmetry axis was reduced by $0.29 \mathrm{~N} / \mathrm{m}$ to $2.35 \mathrm{~N} / \mathrm{m}$.
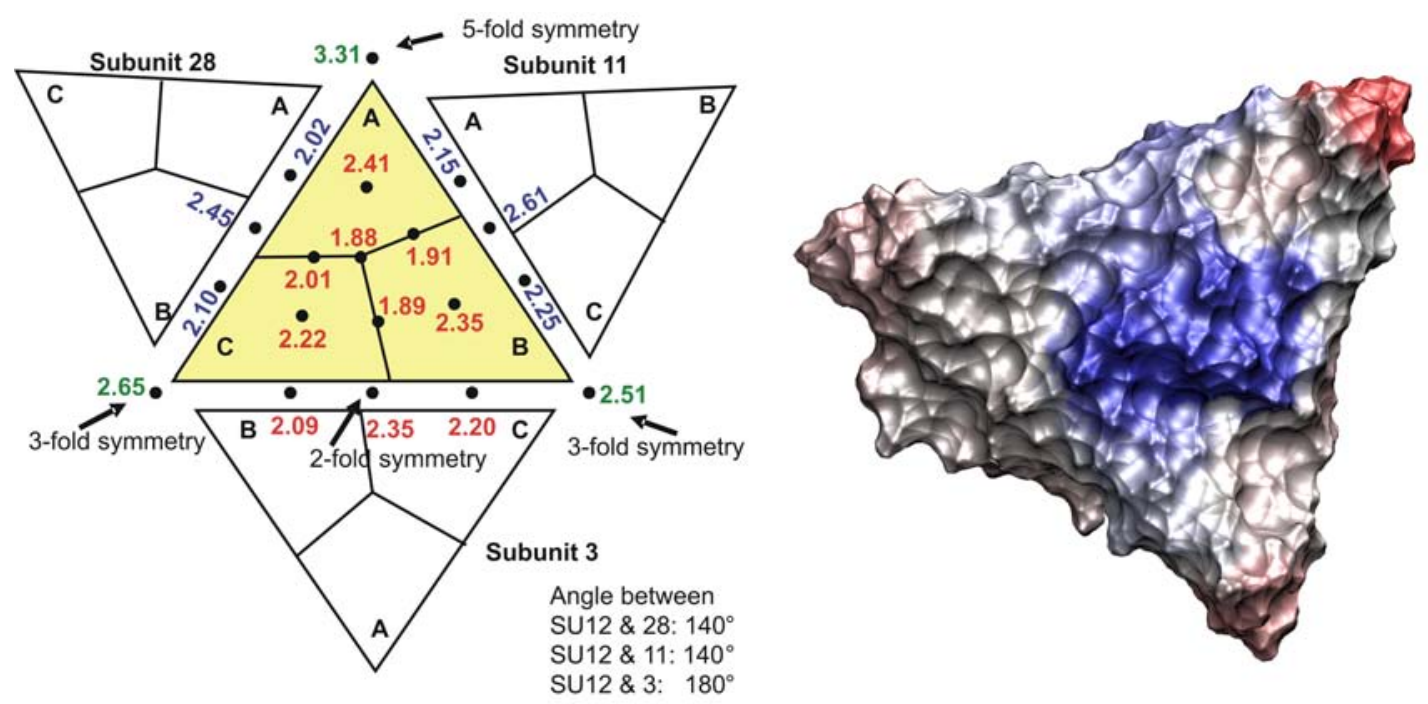

Figure 4.17: Elastic constants of the capsid without calcium, probed from inside. Obtained elastic constants (in $\mathrm{N} / \mathrm{m}$ ) and color-bar shown as in Fig. 4.14.

As an estimate for the standard deviation of the elastic constants, for each of the four force-probe simulation series, the 19 differences to the respective mean values were calculated and found to be in the range between $0.10-0.31 \mathrm{~N} / \mathrm{m}$.

\subsubsection{Yielding forces}

In addition to the elastic constants, we determined the yielding forces $F_{\text {max }}$ for each grid point from the four simulation series, each containing 38 FP simulations towards the same 19 grid points of SBMV as described before. As can be seen from all 152 simulation, the distribution of $F_{\max }$ was more homogeneous than that of the elastic constants with $F_{\text {max }}$ varying between $1.80 \mathrm{nN}$ and $3.80 \mathrm{nN}$ (Fig. 4.18).

\section{Capsid with calcium, probed from outside}

When the tip-sphere was pushed against the outer surface of SBMV including calcium ions in the protein structure, the largest yielding forces were seen at the interfaces between the subunits where two A-proteins meet (SU 12-11: $3.80 \mathrm{nN}$ and $\mathrm{SU}$ 12-28: $3.75 \mathrm{nN})$ and B-C proteins (3.47 $\mathrm{nN}$ and $3.27 \mathrm{nN}$, respectively, Fig. 4.19). The 


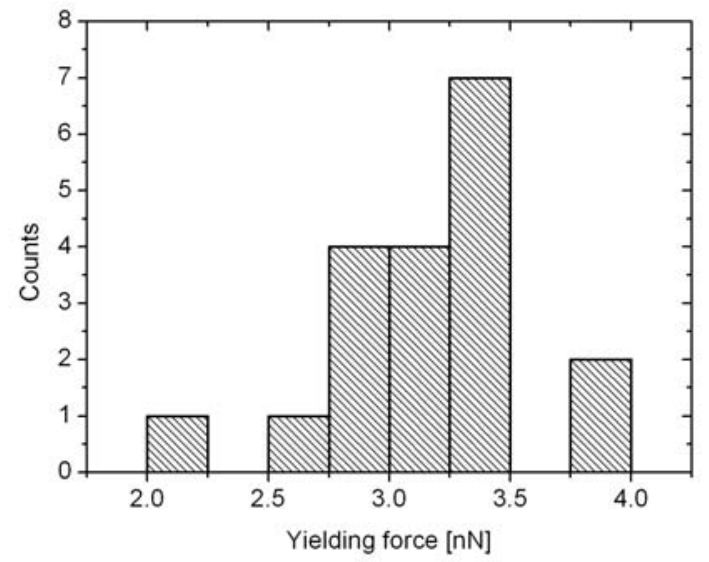

Figure 4.18: Histogram of the yielding forces obtained for the 19 grid points on the outer surface of SU 12 with calcium ions as in Fig. 4.13.

yielding forces were smaller at the interface of subunit $12-3(2.75 \mathrm{nN}$ and $2.79 \mathrm{nN})$ and along the 2-fold symmetry axis $(2.68 \mathrm{nN})$. At the center of the pentamer, a yielding force of $2.96 \mathrm{nN}$ was determined, and $3.36 \mathrm{nN}$ and $3.40 \mathrm{nN}$, respectively, for the two hexamer centers. Within the subunit, $F_{\max }$ varied between $2.99 \mathrm{nN}$ and $3.31 \mathrm{nN}$, with the only exception being the subunit center, which exhibited the smallest yielding force $(2.16 \mathrm{nN})$.
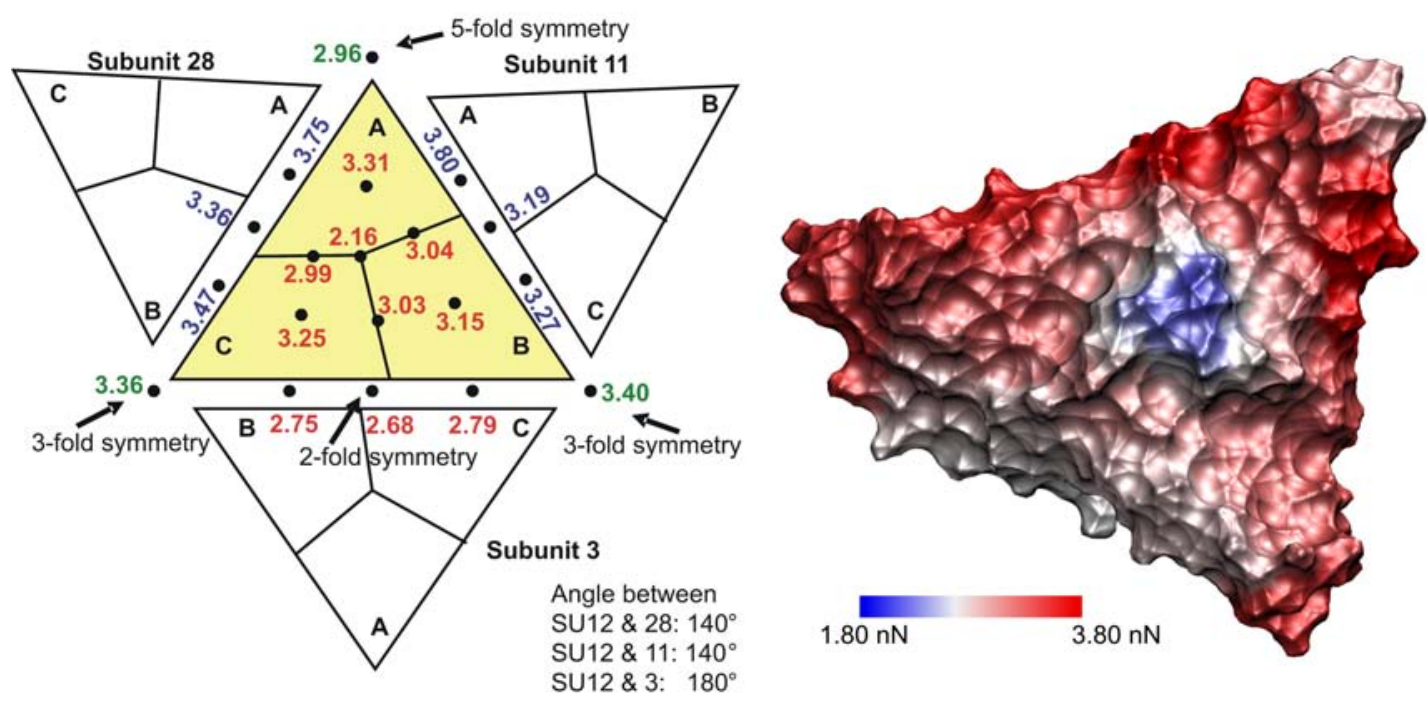

Figure 4.19: Yielding forces of the capsid with calcium, probed from outside. Obtained yielding forces (in $\mathrm{nN}$ ) shown as in Fig. 4.14 (blue: low stability, red: high stability). 


\subsection{Results}

\section{Capsid with calcium, probed from inside}

When the tip-sphere was pushed against the inner shell of the capsid including calcium, the distribution of yielding forces changed drastically (Fig. 4.20). The yielding force determined at the pentamer center dropped to $1.80 \mathrm{nN}$ and became the least stable position on the subunit surface. Also the forces at the hexamer centers decreased to $2.73 \mathrm{nN}$ and $2.59 \mathrm{nN}$, respectively. A stiffness increase was only observed along the 2 -fold symmetry axis $(3.10 \mathrm{nN})$. The interfaces of SU 12 to its adjacent subunits 11 and 28 became softer, and in average, the yielding forces declined by more than $1.0 \mathrm{nN}$. Inside subunit 12, the yielding forces were found to be similar to the values obtained on the outer surface of the subunit with calcium ions.
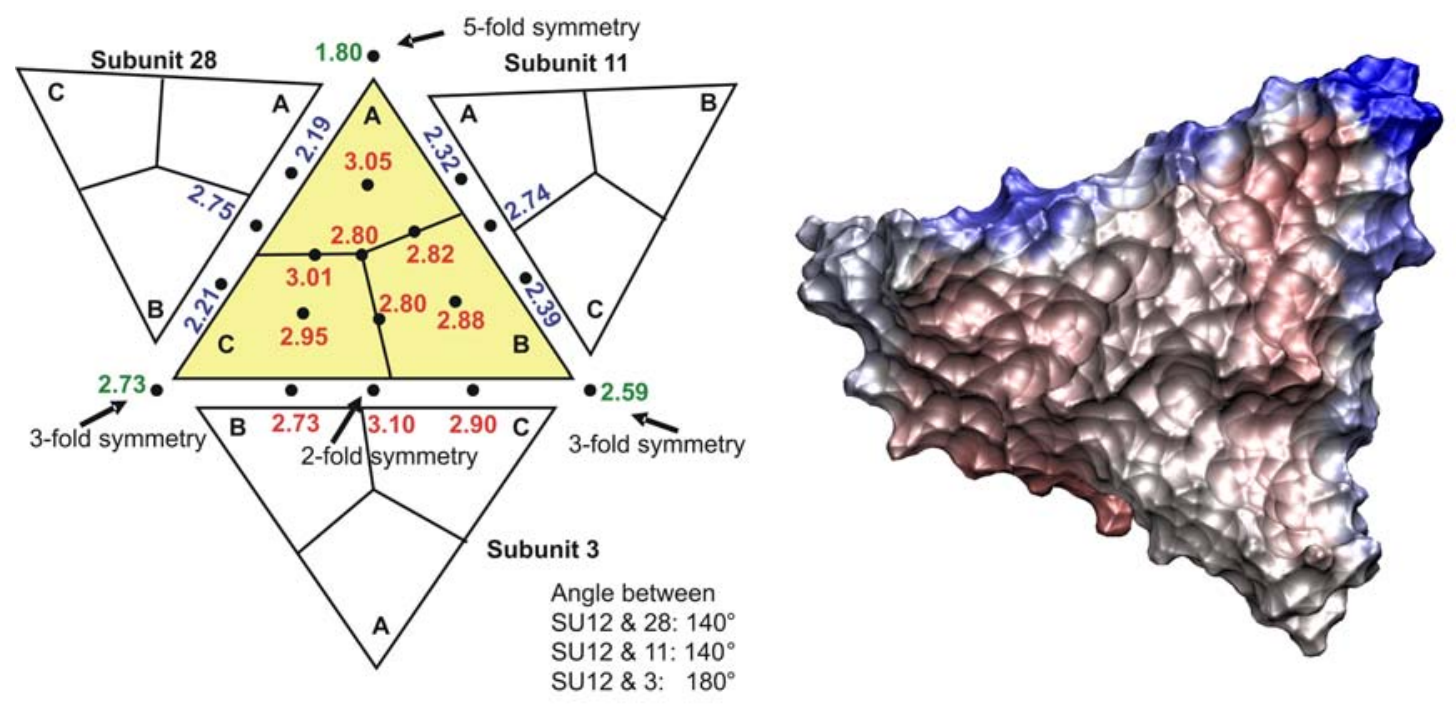

Figure 4.20: Yielding forces of the capsid with calcium, probed from inside. Obtained yielding forces (in $\mathrm{nN}$ ) and color-bar shown as in Fig. 4.19. 


\section{Capsid without calcium, probed from outside}

A dramatic change in the yielding force along the 5-fold symmetry axis was seen when the tip-sphere was pushed against the outer surface of SBMV without $\mathrm{Ca}^{2+}$ ions (Fig. 4.21). The value dropped by $1 / 3$ from nearly $3 \mathrm{nN}$ to $2.04 \mathrm{nN}$, and the pentamer center became the least stable position of the subunit. The yielding forces on the other grid points were hardly affected by the calcium removal and their values barely changed compared to the results obtained on the outer surface of the capsid with calcium ions.
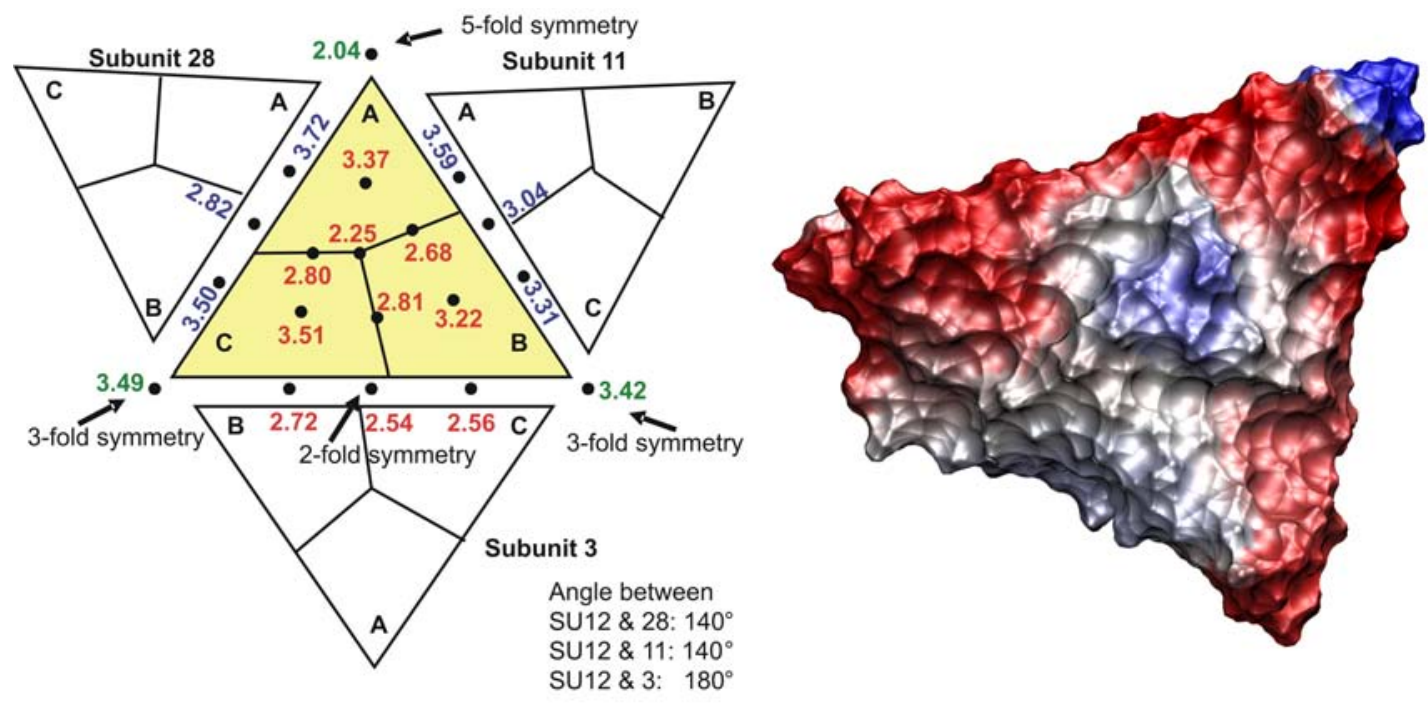

Figure 4.21: Yielding forces of the capsid without calcium, probed from outside. Obtained yielding forces (in $\mathrm{nN}$ ) and color-bar shown as in Fig. 4.19. 


\subsection{Results}

\section{Capsid without calcium, probed from inside}

The distribution of yielding forces on the inner surface of SBMV without $\mathrm{Ca}^{2+}$ ions was found to be similar to that on the inner surface with $\mathrm{Ca}^{2+}$ (Fig. 4.22). The yielding force at the subunit center was slightly reduced by $0.35 \mathrm{nN}$ to $2.45 \mathrm{nN}$, the least stable position was the pentamer center $(2.03 \mathrm{nN})$, as already found from the simulations with $\mathrm{Ca}^{2+}$.
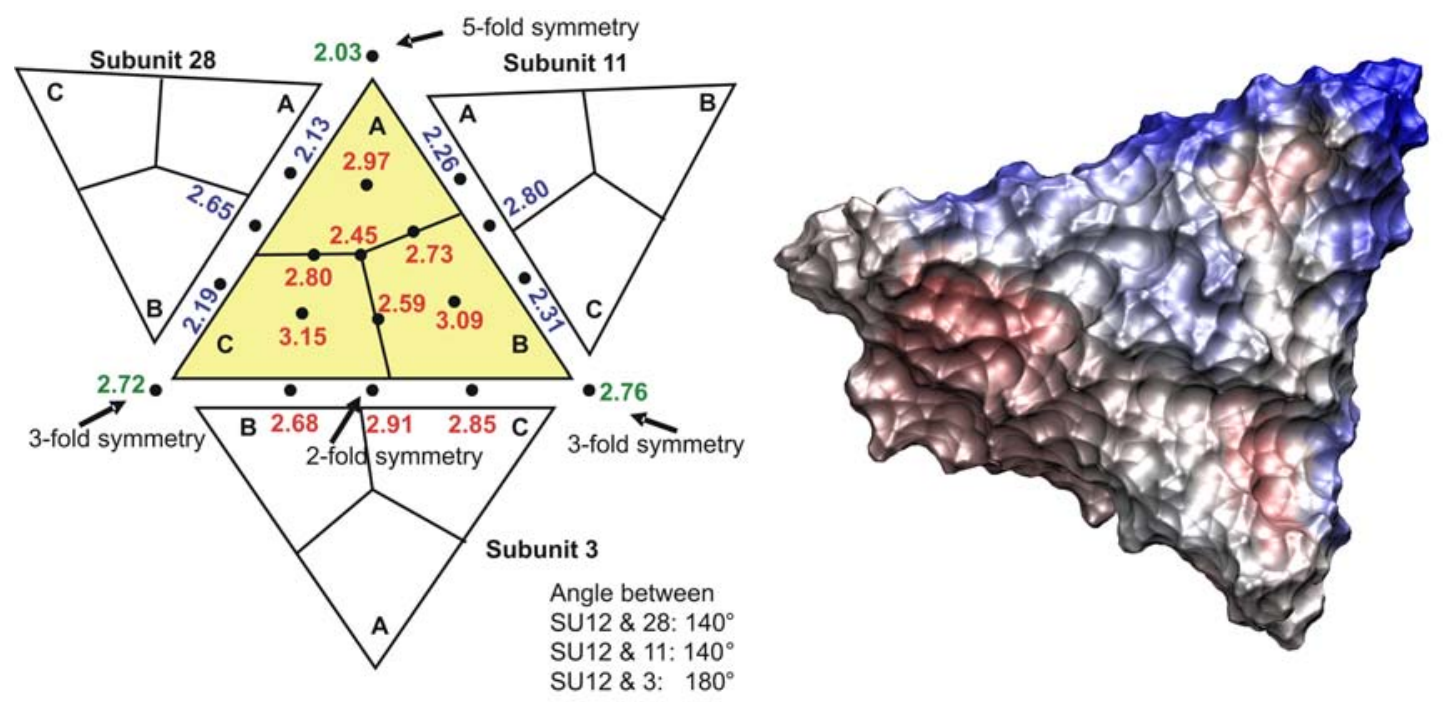

Figure 4.22: Yielding forces of the capsid without calcium, probed from inside. Obtained yielding forces (in $\mathrm{nN}$ ) and color-bar shown as in Fig. 4.19.

The estimated standard deviation, determined from all 152 simulations, was in the range of $0.08-0.30 \mathrm{nN}$. 


\subsection{Discussion}

The transition from the assembled virus to a structure that is able to release the genetic material is accompanied by a change in mechanical properties that enables breaking of the shell or an opening of a port. Without a modification of the elastic constants and yielding forces of the capsid, the mature virus structure remains stable and only external forces or the interaction with the surrounding area could cause an opening of the shell. Experiments showed that the icosahedral shell of Southern Bean Mosaic Virus becomes infectious when the 180 calcium ions are removed from the capsid structure [23]. After removal, the virus swells up to $44 \%$ in volume $[60,23]$, which is the first step in cell infection.

During the $32 \mathrm{~ns}$ equilibration phase of the solvated shell of SBMV without $\mathrm{Ca}^{2+}$, we did not observe a swelling of the capsid. Three explanations are possible to describe this discrepancy to experimental data. First, swelling was reported to take 3 minutes, and our simulations are too short to see this effect. Second, swelling is supported or even caused by the RNA inside the virus which is missing in our simulations. Third, the $\mathrm{pH}$ value was not high enough, because Rayment et al. reported a swelling at $\mathrm{pH}=7.5$ or higher [60].

After the equilibration phase, the difference in the structure of SBMV with and without $\mathrm{Ca}^{2+}$, measured by the root mean square deviation was $2.3 \pm 0.3 \AA$. Although the deviation in the two structures was mainly caused by thermal fluctuations, the mechanical properties of the two capsids varied markedly as described in the following.

To study the mechanical properties of SBMV and the effect of calcium removal on elastic and fracture behavior, we performed force-probe molecular dynamics simulations on the complete shell of SBMV with and without $\mathrm{Ca}^{2+}$ ions. From the linear regime and maximum force in the force-distance plots, a highly heterogeneous distribution was found for both elastic constants and yielding forces on the surface of SBMV. The distributions of mechanical properties varied for the four different FP simulation series in which the tip-sphere was pushed against the inner and outer surface of SBMV with and without $\mathrm{Ca}^{2+}$ ions in the capsid structure. The different elastic behaviors obtained on the inner and outer shell surfaces were first unexpected, because a symmetric elastic medium should exhibit identical elastic properties for indentation deformations performed from the outer to the inner surface or vice versa. It turned out that the inhomogeneous distribution of protein atoms and the spatially inhomogeneous folding motiv of $\beta$-sheets and $\alpha$-helices resulted in different mechanical properties on the inner and outer capsid surface of SBMV.

The distributions of elastic constants cannot be describe with only a single Gaussian function, as suggested from recent AFM results on the empty capsid of minute virus of mice [44]. Much better agreement is obtained with the bimodal distribution observed by Michel et al. [127], who suggest the mechanical properties seen in our simulations as a likely explanation for their results. As a cautionary remark we note that direct comparison of the distributions of elastic constants is complicated by the fact that the heterogeneous distribution found in our simulations is caused 


\subsection{Discussion}

by structural differences of the capsid on an atomic level, whereas a heterogeneous distribution of elastic constants obtained from AFM experiments might originate from differing mechanical behaviors of capsomers. Remaining differences between the distributions of elastic constants may result from differing tip sizes and geometries, differing accuracies in targeting specific surface regimes or different time scales, or statistical scatter due to the limited number of force probe simulations.

Our force-probe simulations showed that the values for the determined elastic constants and yielding forces on grid points within the subunit were very similar on the inner and outer subunit surface, and are barely affected by the removal of $\mathrm{Ca}^{2+}$. The only exception was seen at the subunit center along the quasi-3-fold symmetry axis. Although the elastic constants obtained for the subunit center varied on the inner and outer surface and changed with $\mathrm{Ca}^{2+}$ removal, this grid point was always found to be the weakest or close to the weakest position of the capsid.

Similar observations were made for the yielding forces. When the tip-sphere was pushed against the subunit center on the outer surface (SBMV with and without $\mathrm{Ca}^{2+}$ ), this position always exhibited a weak stability. When the tip-sphere approached this grid point from inside the virus, the stability increased for the structure with $\mathrm{Ca}^{2+}$ compared to the capsid without ions.

On the subunit interfaces and along the symmetry axis, the mechanical properties varied markedly on the inner and outer surface, and changed when $\mathrm{Ca}^{2+}$ ions were removed. The variations and distributions of elastic constants and yielding forces were not correlated because they describe different material properties.

We have seen from various simulations in which the tip-sphere was directed against the subunit proteins that the $\beta$-sheet region of the subunit proteins showed a higher stiffness than the $\alpha$-helices and loops. Similar observation were also made by Ackbarow et al. from studies of a model protein structure proposed for Alzheimer's amyloid $\beta$-fibrils [128].

The elastic constants between two A-proteins on the outer grid points were consistently larger than the values obtained at the interface between the subunits where protein $\mathrm{B}$ and $\mathrm{C}$ meet. We attribute this trend to (at least) two effects: First, purely geometrical properties might render the pentamers stiffer than the hexamers [129], an effect which might extend to the interaction of the A-proteins. Second, Reddy et al. [130] calculated the dimer association energies for SBMV, where also a larger energy was found for the interaction between the A-proteins $(-78.0 \mathrm{kcal} / \mathrm{mol})$ than for the $\mathrm{B}-\mathrm{C}$ interaction $(-74.0 \mathrm{kcal} / \mathrm{mol})$, which might also contribute to the higher stiffness between A-A than between B-C proteins.

From all simulations we see the largest elastic constant at the pentamer center except when the tip-sphere was pushed towards the inner surface of SBMV with $\mathrm{Ca}^{2+}$. Tama et al. observed a larger flexibility for pentamers than for hexamers of SBMV [26], which result cannot be directly related to the obtained elastic constants in a straight forward manner. A different behavior was observed by Carrasco et al. from AFM experiments on the capsid of minute virus of mice [44], who, besides the homogeneous distribution discussed before for the empty capsid, reported the largest elastic constant at the 2-fold axes and the weakest at the pentamer center 
for full capsids. Further investigations on the stability of hexamers and pentamers are described in chapter 6.1.

For Human Rhinovirus 14, an icosahedral $\mathrm{T}=3$ virus with very similar radial positions of the $\mathrm{C}_{\alpha}$ atoms and tertionary fold of the main protomer proteins compared to the structure of SBMV [131], Giranda et al. [27] suggested that pentamers open to release the genetic material during infection. The same proposal was made by Mosser et al. three years later for poliovirus type 3 [28]. We propose that fracture of viral shells should occur at capsid positions that exhibit small yielding forces compared to their surrounding areas. The distribution of elastic constants, and thus the stiffness of the viral shell has a minor effect on the rupture position. A possible port for RNA release of SBMV cannot be proposed from changes of the elastic constants, only from variation of the yielding forces after calcium removal.

Therefore, a large yielding force and, thus, a high stability of the pentamer center was obtained from the simulation in which the tip-sphere was pushed from outside against the structure with $\mathrm{Ca}^{2+}$. The stability of the pentamer center dropped markedly due to the removal of calcium ions - here the pentamer center became the least stable position on the outer surface of the capsid. The yielding forces determined at the remaining grid point positions on the outer shell surface hardly changed. On the inner capsid surface, the weakest stability of the shell with and without $\mathrm{Ca}^{2+}$ was found along the 5-fold symmetry axis. The observed changes in the distribution of yielding forces let us draw the following conclusions. 


\subsection{Conclusion}

Experiments showed that SBMV becomes infectious after removal of the calcium ions [23]. Comparison of the obtained values for the elastic constants and yielding forces and their distributions on the inner and outer surface on the capsid with and without $\mathrm{Ca}^{2+}$ make it possible to predict a capsid position that breaks open to release the RNA after $\mathrm{Ca}^{2+}$ removal.

When RNA exerts a force to the inner surface of the shell, capsid rupture would occur at the pentamer center after calcium removal because the smallest yielding force has to be applied along the 5-fold symmetry axis to break the capsid open. Although large elastic constants were obtained at the pentamer center on the inner and outer capsid surface, fracture behavior is mainly determined by the distribution of yielding forces, and we therefore propose an opening of the pentamer centers after calcium removal.

Removal of the calcium ions from the capsid resulted in a significant softening along the 5 -fold symmetry on the outer pentamer surface which became the weakest capsid position on the outer surface. Therefore, fracture of the shell due to external forces would occur at the pentamer center as well, as also proposed from the forces obtained on the inner capsid surface. Such an external force could arise from interactions of the cell membrane with the viral shell [125], which would also support our proposed scenario for RNA release along the 5-fold symmetry axis.

The yielding forces obtained at the 3 - and 2-fold symmetry axes are higher compared to the surrounding subunit surface and the pentamer center. Therefore, capsid breakage along these symmetry axes is improbable. Small elastic constants and yielding forces on the capsids with and without calcium ions were found at the subunit center. If the subunit center acts as a possible port for RNA release, then the mature virus structure would already be unstable and could infect cells without the removal of calcium ions because the mechanical properties of the subunit center are very similar for the virus structure with and without $\mathrm{Ca}^{2+}$. But capsid breakage and release of the genetic material without preceding calcium removal was not observed in experiments. Therefore, we also consider rupture at the positions along the 3and 2-fold symmetry axes unlikely for RNA release. Additionally, an RNA release, in which the RNA strand is pushed or pulled through one of the subunit proteins, can be neglected due to the high stability of the $\beta$-barrel. Our results clearly show that only pentamers could act as possible ports for RNA during dematuration and cell infection.

Finally, the answer where and how RNA is released can only be made from experiments. As it is impossible to probe the mechanical properties on the inner surface of SBMV from AFM experiments, our simulations reveal the first insights into the distribution of mechanical properties on the outer and inner surface of an icosahedral viral shell on atomic length scales from which predictions for a possible port for RNA release have been obtained. 


\section{Structural dynamics of SBMV during deformation}

\subsection{Introduction}

Besides the elastic constants and yielding forces discussed in detail in the previous chapter, the structural dynamics of the viral shell during indentation remain unknown, as well as the origin of elastic deformation and fracture behavior, and the questions arise

- What happened to the protein structure when the tip-sphere indented the viral shell?

- What can the force-distance curves tell us about the deformation behavior of the capsid?

- Could plastic deformation be observed in the simulations? If yes: When did the cross-over from elastic to plastic deformation take place, and how could such a transition be understood in structural terms?

- How do the mechanical properties depend on the size of the tip-sphere?

- How do elastic constants and yielding forces change with probe-velocity?

In order to answer these questions, this chapter focuses on the deformation dynamics of the shell of Southern Bean Mosaic Virus (with calcium in the structure) during indentation of protein A, SU 12, with the tip-sphere. The deformation of the capsid of SBMV on length scales of the complete viral shell was investigated, as well as structural changes on atomic level from which we were able to find answers to all these questions.

For both, elastic constants and yielding forces, a logarithmic velocity dependence was seen over nearly two decades, the explanation of which requires including nonequilibrium effects perpendicular to the reaction coordinate within the established theory of enforced barrier crossing. This assumption is corroborated using Monte Carlo simulations, in which a particle was pushed through a harmonic energy landscape with relaxation dynamics taking place perpendicular to the reaction coordinate.

Furthermore, friction and surface effects that might contribute to the obtained elastic constants and yielding forces were investigated, as well as water flux through the viral shell during deformation. 


\subsection{Methods}

The simulation set up for SBMV with calcium ions in the capsid structure is described in chapter 4.2.1, as well as the force-probe simulations in 4.2.2. Additionally, $38 \mathrm{FP}$ simulations towards each of the 19 grid points on the outer surface were performed with a probe-velocity of the tip-sphere of $0.05 \mathrm{~nm} / \mathrm{ps}$. To study the variation of the forces with probe-velocity in more detail, a series of simulations along the 5 -fold symmetry axis towards the center of a pentamer was performed with nine different probe-velocities, ranging from $0.001 \mathrm{~nm} / \mathrm{ps}$ to $0.05 \mathrm{~nm} / \mathrm{ps}$. All force-probe simulations with a probe-velocity of $0.05 \mathrm{~nm} / \mathrm{ps}$ were performed twice, whereas the simulations, in which the tip-sphere was pushed against the pentamer center, were performed five times. The first series of force-probe simulations was started from structures extracted after $12 \mathrm{~ns}$ of the equilibration run, the second after $12.25 \mathrm{~ns}$, the third after $12.5 \mathrm{~ns}$, the fourth after $12.75 \mathrm{~ns}$, and the fifth after $13 \mathrm{~ns}$.

To study the relaxation behavior of the capsid, for a number of selected simulations the pushing potential acting upon the tip-sphere was switched off at different times. Subsequently, the system was equilibrated for 500-1000 ps with free MD simulations. The Monte Carlo simulation technique is described in the Results and Discussions part of this chapter for better understanding. 


\subsection{Results and Discussions}

\subsubsection{Regimes of deformation}

When the tip-sphere was pushed against the viral shell, the force acting on the tipsphere was monitored. Figure 5.1 shows a typical force-distance (red) and the corresponding force-time curve (blue), taken from a simulation in which the tip-sphere was pushed towards the center of mass of protein A of SU 12 with a probe-velocity of $0.01 \mathrm{~nm} / \mathrm{ps}$. At the beginning of the simulation, the tip-sphere moved through the water for $120 \mathrm{ps}$ before it touched the capsid, at which point the force increased. As shown in Fig. 5.1, three regimes can be distinguished, a pre-linear regime (1), a linear regime (2), and a rearrangement regime (3).

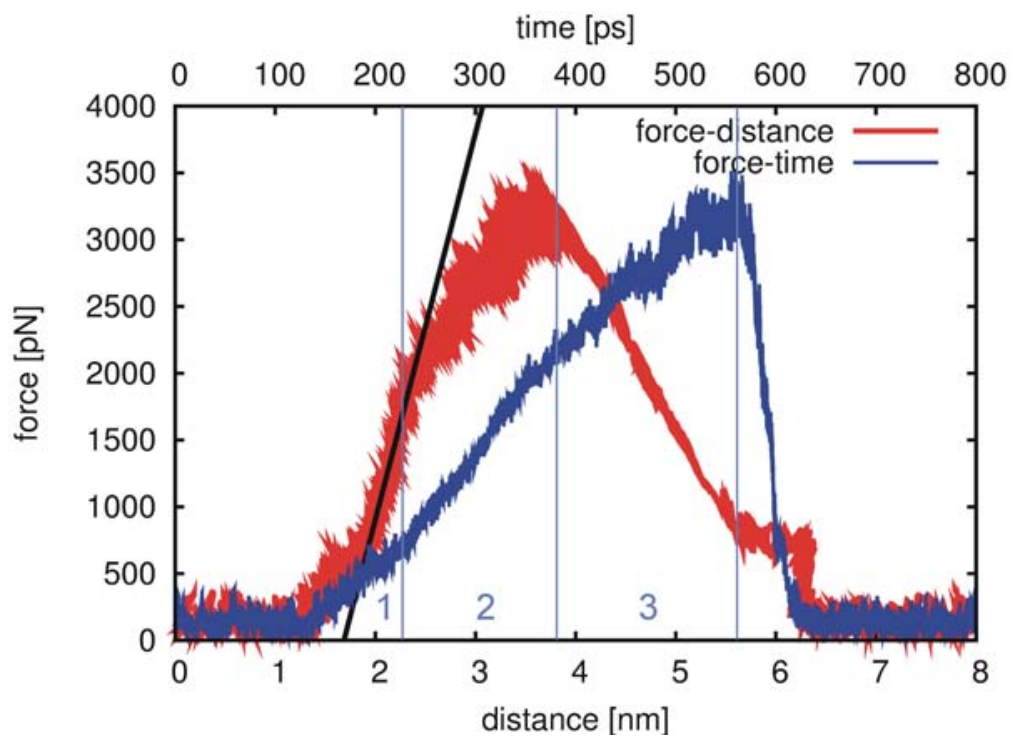

Figure 5.1: Force, acting on the tip-sphere, as a function of time (blue) and distance (red) during a typical force-probe simulation in which the tip-sphere was pushed against protein A of SU 12 with a probe-velocity of $0.01 \mathrm{~nm} / \mathrm{ps}$. Three regimes can be distinguished, 1) a pre-linear regime, 2) a linear regime and 3) a rearrangement regime. The black line depicts the slope in the force-distance plot, from which the elastic constant (in $\mathrm{N} / \mathrm{m}$ ) is determined.

The pre-linear regime is characterized by small forces, heterogeneously distributed over the surface. These forces originate from local deformations of an outer capsid layer consisting mainly of loops and $\beta$-strands. When the tip-sphere initially touched the capsid, the first layer was pushed towards the viral center, thereby approaching underlying layers. The subsequent contact between the two layers marks the transition from the pre-linear (1) to the linear regime (2). The linear regime is characterized by elastic deformation of the viral shell. The slope in the force-distance 
curve from 220-390 ps determines the elastic constant of the capsid at the respective position. After approx. $390 \mathrm{ps}$, the force bended over to a nonlinear behavior and finally leveled off at $560 \mathrm{ps}$ at a yielding force of $F_{\max }=3310 \mathrm{pN}$. Subsequently, the tip-sphere was pushed inside the virus and left the shell, resulting in a rapid force decline. Remarkably, substantially increased fluctuations were observed in the rearrangement regime, which, together with the mechanical properties, will be analyzed in structural terms later below.

These three regimes were observed for all 19 grid points. Due to the heterogeneity of the protein structure and its outer layer, the pre-linear regime was less pronounced, e.g. for the grid point between protein B and C of SU 12. Only for one force-probe simulation in which the tip-sphere was directed towards the $\beta$-sheet region of protein A (Fig. 4.12), the prelinear regime was not seen because only at this position on the capsid surface no flexible loops were present. Furthermore, in several cases the length of the rearrangement regime was much shorter than shown in Fig. 5.1, e.g., only a few picoseconds as for an approach along the 5-fold symmetry axis.

\subsubsection{Water permeation, friction and surface effects}

During the equilibration phase and also during the force-probe simulations, no water permeated the capsid. This observation agrees with results by Silva et al. [125], who reported a high energy barrier for water of $E=300 \mathrm{kcal} / \mathrm{mol}$ at the gate along the 5 -fold symmetry axis. No other position on the protein shell exhibited a gate for possible water or ion permeation, which is in good agreement with the lack of water flux observed in our simulations. The resulting compression of the water volume inside the capsid during indentation raises the question, however, to which extent the observed forces actually reflect the mechanical properties of the shell.

To address this question, we estimated the force arising from the water compression. When the shell was indented by the tip-sphere, an area of the size of one subunit $\left(A \approx 42 \mathrm{~nm}^{2}\right)$ was pushed into the capsid and reduced the inner virus volume by $\sim 3.5 \mathrm{~nm}^{3}$. This estimated volume change was derived from the change of the radius of gyration of the capsid (Fig. 5.5) and the distance the subunit was pushed into the capsid (Fig. 5.7 and 5.11). Assuming a compressibility of TIP4P water of $\kappa=67 \cdot 10^{-11} \mathrm{bar}^{-1}$ [132], the inner capsid pressure is expected to increase from 1 bar to $p \sim 5.43$ bar at the yielding point, resulting in a force $F=p \cdot A=22.8 \mathrm{pN}$; i.e., approximately $1 \%$ of the obtained yielding force. Therefore, the water compression is not expected to affect the observed forces. 


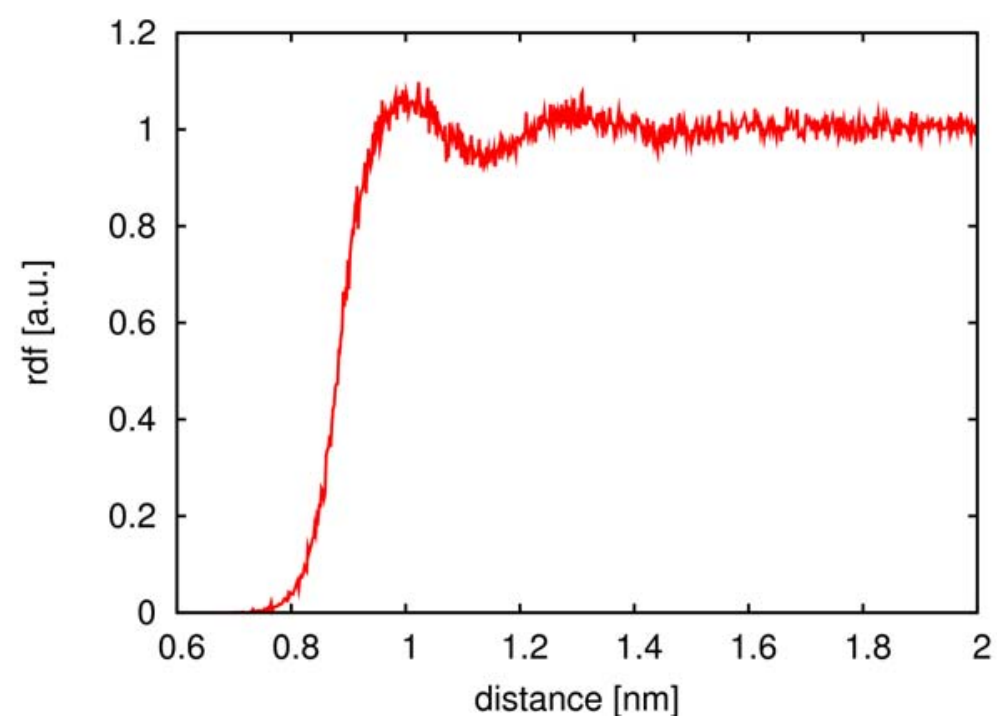

Figure 5.2: Radial distribution function (rdf) of the tip-sphere with the surrounding oxygen atoms from the TIP4P water molecules. Here the tip-sphere was pushed through the bulk water with a push-velocity of $0.01 \mathrm{~nm} / \mathrm{ps}$.

Because of the relatively high probe-velocity (compared to those used in typical AFM experiments) which has necessarily been used in our simulations, frictional forces might also contribute to the observed force-time and force-distance curves. To separate these frictional forces from the mechanical properties of interest, we performed six force probe simulations in which the tip-sphere was pushed through bulk water with probe-velocities between $0.0001 \mathrm{~nm} / \mathrm{ps}$ and $0.05 \mathrm{~nm} / \mathrm{ps}$. As expected from the Stokes equation, a linear increase of the observed frictional force with probevelocity was seen. The resulting slope of $5.63 \pm 0.14 \mathrm{nN} \cdot \mathrm{s} / \mathrm{m}$ was somewhat smaller than the value from the respective Stokes equation, $\xi=6 \pi \eta r=6.59 \mathrm{nN} \cdot \mathrm{s} / \mathrm{m}$, for TIP4P water [133], assuming stick boundary conditions between solvent and tipsphere [134]. The deviation arises from the Stokes radius that was determined as the minimum distance $(0.70 \mathrm{~nm}$, determined from a radial distribution function, Fig. 5.2) between the tip-sphere center and the water molecules. For the applied probe-velocity of $0.01 \mathrm{~nm} / \mathrm{ps}$, the frictional force found in the simulation $\left(F_{\text {sim }}=\right.$ $65.2 \pm 1.5 \mathrm{pN})$ agrees very well with the force calculated from the Stokes equation $\left(F_{\text {Stoke }}=65.9 \mathrm{pN}\right.$, Fig. 5.3). We note that stick boundary conditions were used rather than slip boundary condition [135] - here the Stokes equation changes to $\xi=4 \pi \eta r$ - because the latter has been shown to apply only to solutes much larger than the used tip-sphere [135]. In summary, the obtained frictional forces are small with respect to the observed elastic forces. In particular, we consider our probevelocity sufficiently slow such that friction can be neglected. 


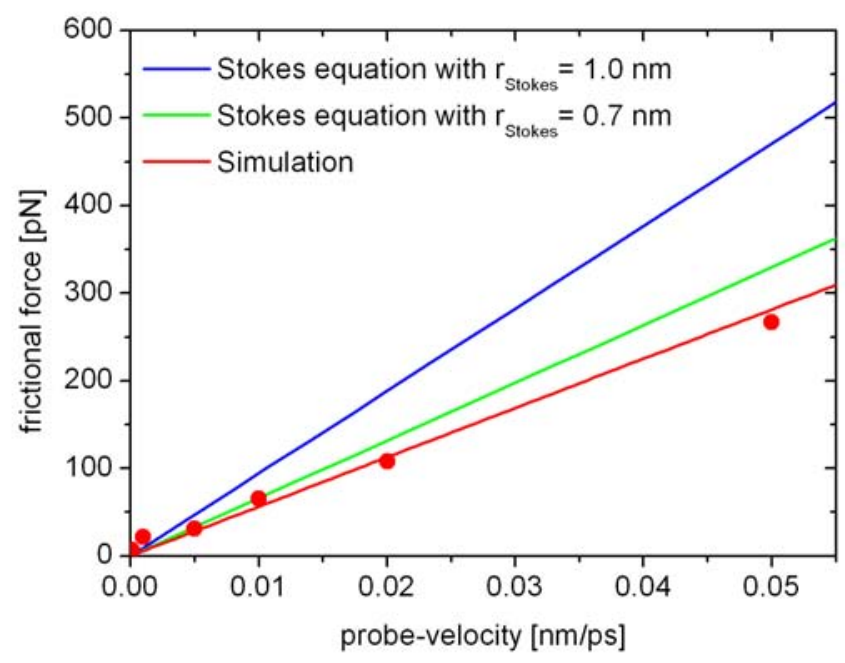

Figure 5.3: Frictional forces as a function of probe-velocity. The data were obtained from force-probe simulations shown as red dots connected via a fitting function from linear regression (red line). The green and blue lines depict the theoretical values calculated from the Stokes equation for two tip-sphere radii. Stick-boundary conditions between solvent and solute atoms were assumed.

To study to which extent the free energy increases due to a possible increase of the solvent accessible hydrophobic area (SAS) of the capsid and contributes to the observed force increase during indentation, this surface area was recorded during the force-probe simulations. During deformation of the capsid in the pre-linear, linear and the rearrangement regime until 480 ps (Fig. 5.1), the capsid SAS area for the hydrophobic and hydrophilic part remained constant within fluctuations of $\Delta \mathrm{A}=2.0 \mathrm{~nm}^{2}$. Subsequently, when the force fluctuations in the force-distance plot increase markedly (Fig. 5.1), the SAS area increased by approx. $\Delta A=10.0 \mathrm{~nm}^{2}$ up to the point of yielding (480 ps - $560 \mathrm{ps}$, Fig. 5.4). Assuming proportionality between the SAS area and the surface free energy $\Delta G$ with a proportionality constant of $24 \mathrm{cal} / \mathrm{mol} \cdot \AA^{2}[136,137]$, we determined the respective free energy increase and the resulting forces $F$ along the indentation path length $\Delta x$ needed to change the hydrophobic surface area. For $\Delta \mathrm{A}=2 \mathrm{~nm}^{2}$ a surface free energy of about $\Delta G=$ $3.3 \cdot 10^{-20} \mathrm{~J}$ was found, resulting in a force of $\Delta G / \Delta x=F \approx 22 \mathrm{pN}$ with $\Delta x=1.5 \mathrm{~nm}$ (Fig. 5.1, blue curve), which is $33 \%$ of the frictional forces. At this point of yielding the surface free energy increased to $\Delta G=16.7 \cdot 10^{-20} \mathrm{~J}$ and the resulting force to $F \approx 210 \mathrm{pN}(\Delta x=0.8 \mathrm{~nm})$. To conclude, changes in the surface free energy contribute to about $10 \%$ of the yielding force. 


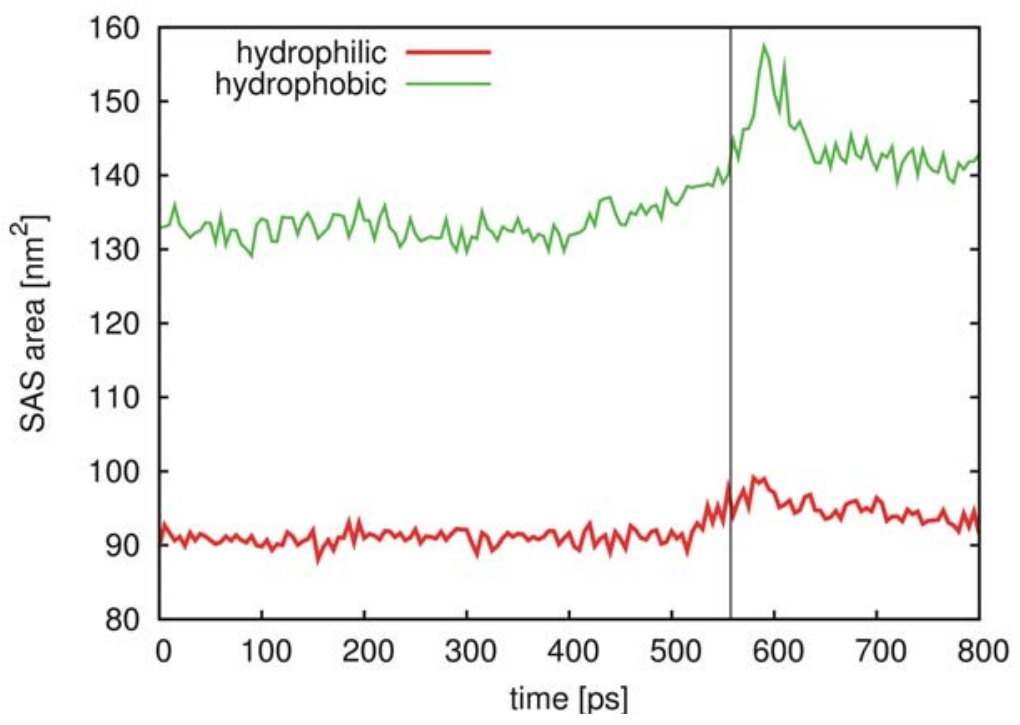

Figure 5.4: Solvent accessible surface (SAS) area of SU 12 during indentation of protein A. Only the SAS of SU 12 changes during this force-probe simulation, the remaining capsid surface was unaffected. 


\subsubsection{Structural characterization of deformation}

To structurally characterize the deformation of the viral shell during the simulation and to explain its mechanical behavior in the rearrangement regime, we chose the FPMD simulation in which the tip-sphere was pushed through the center of mass $(\mathrm{COM})$ of protein A, SU 12 with probe-velocity $0.01 \mathrm{~nm} / \mathrm{ps}$. We first calculated the radius of gyration of the capsid during deformation (Fig. 5.5).

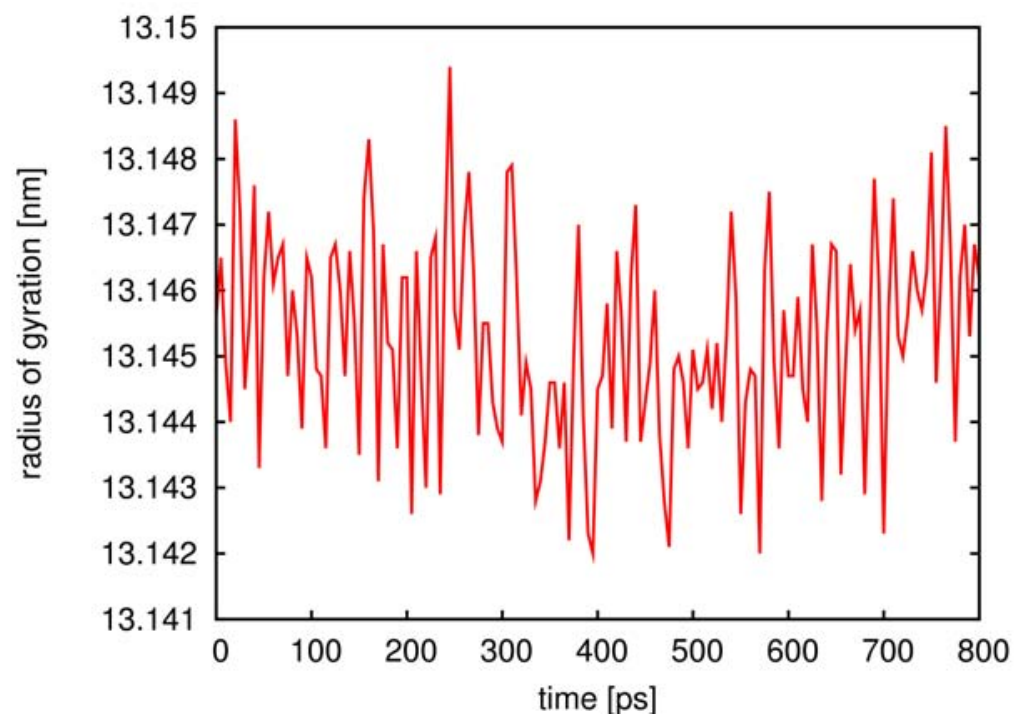

Figure 5.5: Radius of gyration of the viral shell as a function of time for pushing the tip-sphere towards protein A of SU 12. The yielding force was reached at $\sim 560$ ps.

It turned out that the radius of gyration barely changed during indentation of the viral shell with the tip-sphere. Furthermore, the trajectories of the protein atoms and the tip-sphere were monitored; the single residues of the capsid were color-coded for their deformation. As can be seen in Fig. 5.6, deformation was restricted to an area of a few $\mathrm{nm}^{2}$ of the capsid. The strongest deformation, measured as an rmsd of $2.5 \mathrm{~nm}$ (see Fig. 5.7 below) occurred only on a few $\AA^{2}$ area, whereas the size of the induced "defect" on the viral shell was small compared to the complete capsid. Together with the very small decrease of the radius of gyration from $13.146 \mathrm{~nm}$ before the tip-sphere approached the shell to $13.144 \mathrm{~nm}$ at yielding, we conclude that the spherical shape of the capsid hardly changed during indentation and the virus shell was compressed only slightly. 

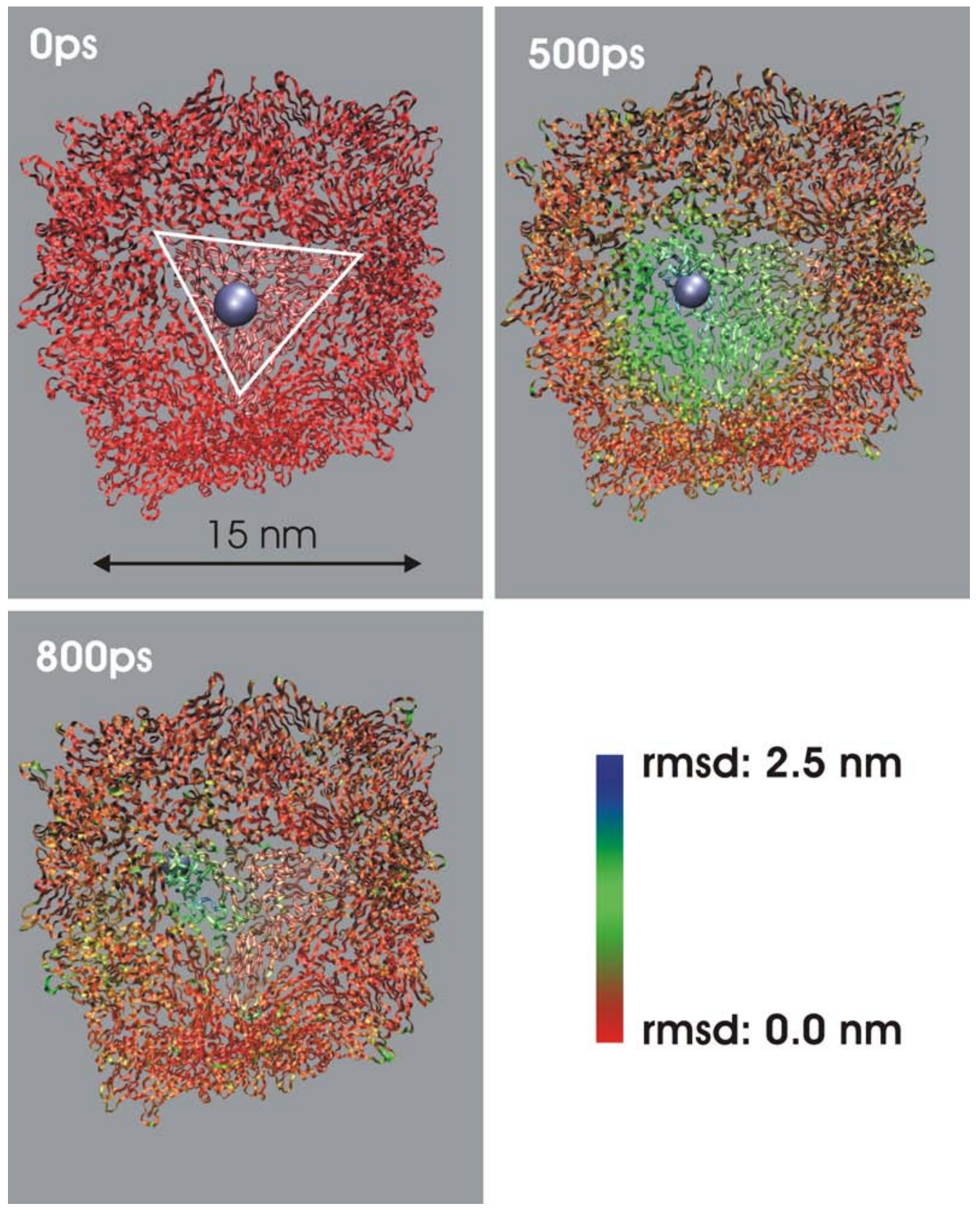

Figure 5.6: Subunit 12 (emphasized by the white line in the ribbon structure and the white triangle) and its adjacent subunits. The tip-sphere which was pushed against protein A, is shown as a grey sphere. Each residue is color-coded for its deformation as described in chapter 3.3 (red: no deformation, blue: highest deformation). 
Next, we calculated the distance between the COM of SU 12 and the center of the viral shell (Fig. 5.7) to investigate the deformation behavior of the indented subunit.

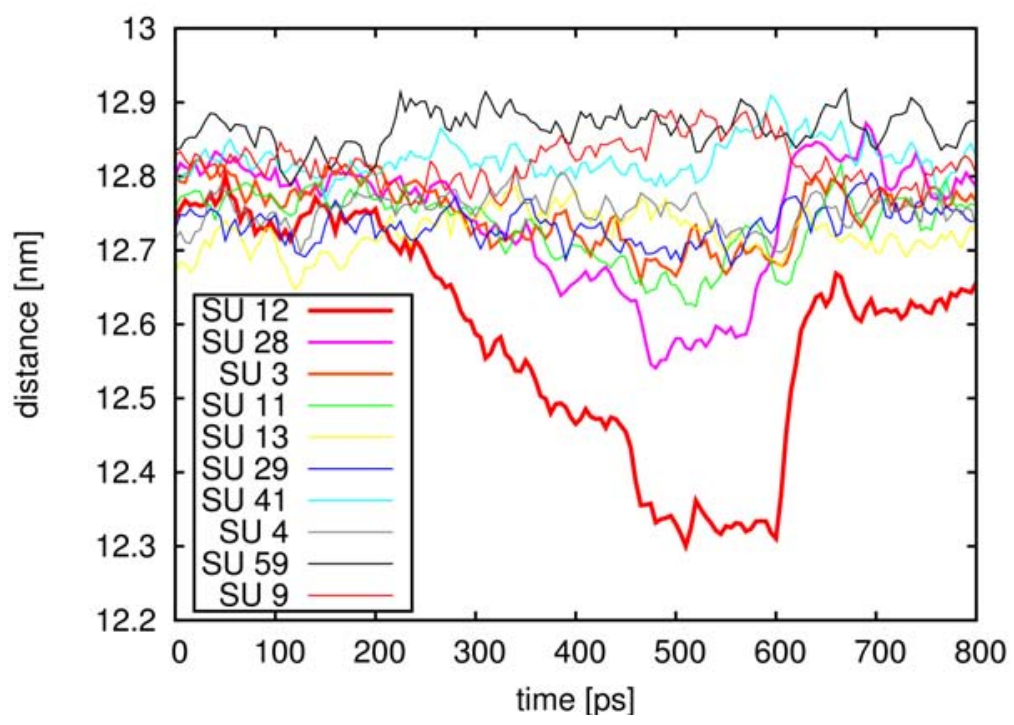

Figure 5.7: Distances between the center of mass (COM) of SU12 and adjacent subunits with the COM of the complete capsid during deformation.

During deformation, the subunit was pushed towards the center of the capsid by $0.45 \mathrm{~nm}$ at the yielding point. Subsequently, the tip-sphere penetrated the protein and the subunit snapped back to its initial position. Also the root mean square deviation (rmsd) of SU 12 with respect to the initial structure increased to $1.4 \mathrm{~nm}$ at 560 ps (yielding point) and subsequently dropped to nearly its initial value after the tip-sphere left the viral shell towards the capsid center (Fig. 5.8). We suggest that, except for the few residues discussed below, the capsid fully recovered its original shape when the external force was switched off during deformation (5.8, colored lines), as well as after complete penetration (5.8, red and black lines). SU 12 thus seems to be highly elastic during the complete deformation phase, even within the regime that we termed "rearrangement regime". Although at this point the origin of the non-linearity and the increase fluctuations in the force-distance curve remains elusive, the above result suggests that any non-elastic behavior will be restricted to a small regime of the subunit.

In the following step, we analyzed the propagation of the protein deformation by monitoring the distance change of the adjacent subunits 11, 28, and 3 of SU 12 with respect to the capsid center and their rmsd compared to the initial shell structure (Fig. 5.8 and 5.9). In contrast to the marked deformation of SU 12, its neighbors deformed only slightly, with the rmsd of SU 28 rising only by $0.25 \mathrm{~nm}$ at $560 \mathrm{ps}$, then immediately returning to its initial value (Fig. 5.9). The rmsd of SU 3 did not change at all beyond thermal fluctuations $(\sim 1.0 \AA)$. We therefore conclude that elas- 


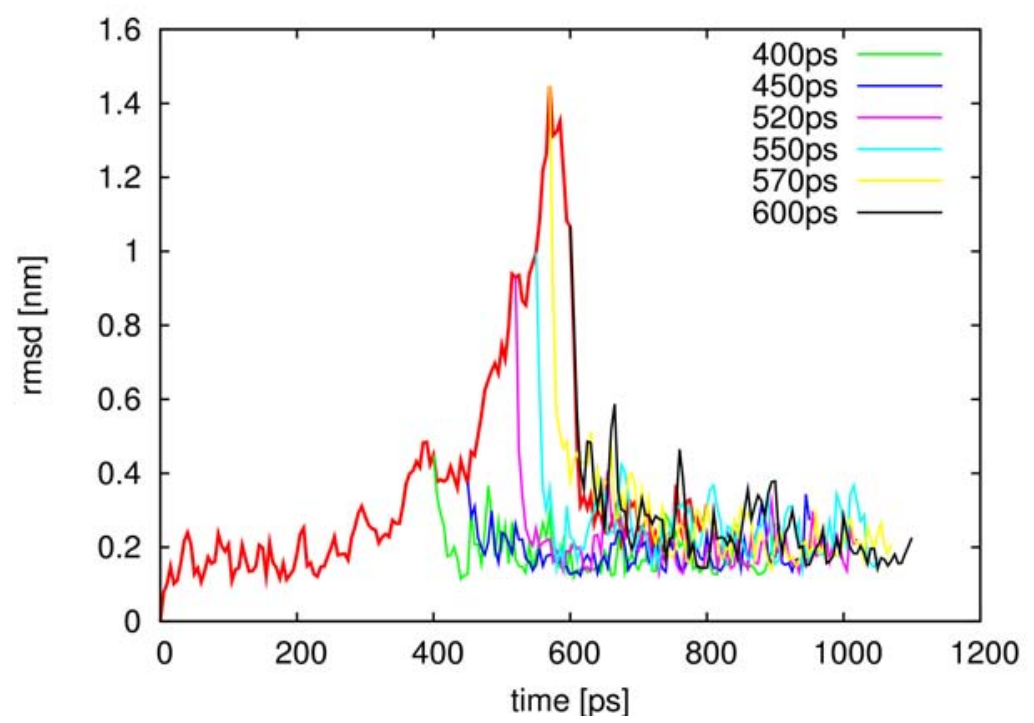

Figure 5.8: Rmsd for SU12 with respect to the initial capsid structure during indentation of protein A (red curve). The simulation was stopped at different time steps, and the system was equilibrated for $1.0 \mathrm{~ns}$. The corresponding rmsd plots are shown by the colored curves.

tic deformation during pushing the tip-sphere towards SU 12 is spatially restricted to the SU 12 region and hardly affects the remaining viral shell.

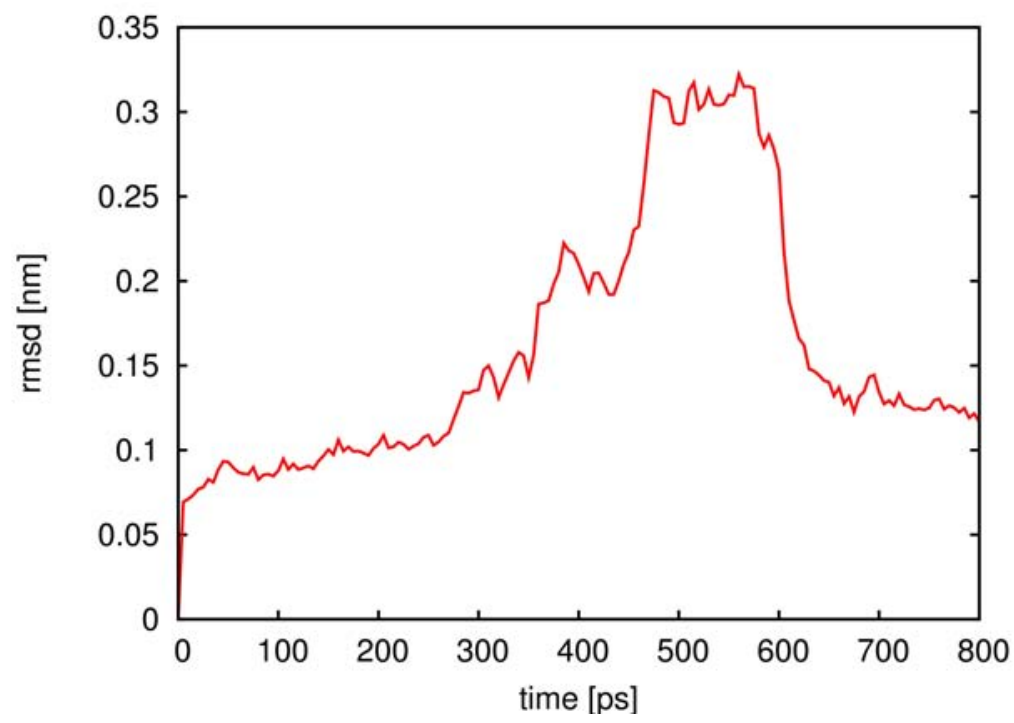

Figure 5.9: Rmsd of SU 28 fitted on the initial capsid structure during indentation of protein A, SU 12. The location of SU 28 with respect to SU 12 is given in Fig. 3.5. 
In order to characterize the deformation on the atomic level, we determined the rmsd of every residue (amino acid) of SU 12, fitted on the starting structure of the whole capsid. The maximum value of the rmsd of every residue was then plotted as a function of residue number. As can seen in Fig. 5.10, the average maximum rmsd for protein $\mathrm{B}$ and $\mathrm{C}$ residues is about $0.5 \mathrm{~nm}$ due to the fact that the subunit was pressed towards the viral center by $0.45 \mathrm{~nm}$ as mentioned above. The average maximum rmsd of protein $\mathrm{A}$ is twice as large compared to protein $\mathrm{B}$ and $\mathrm{C}$, whereas only a few residues (residue 33 and 51) exhibit a maximum rmsd of more than $2.2 \mathrm{~nm}$. Compared to the rmsd calculated for the complete SU 12 mentioned above, we suggest that the subunit rmsd of $1.4 \mathrm{~nm}$ was induced by deformation of only a few residues of protein A which were directly touched or close to the tip-sphere in the simulation (Fig. 5.11).

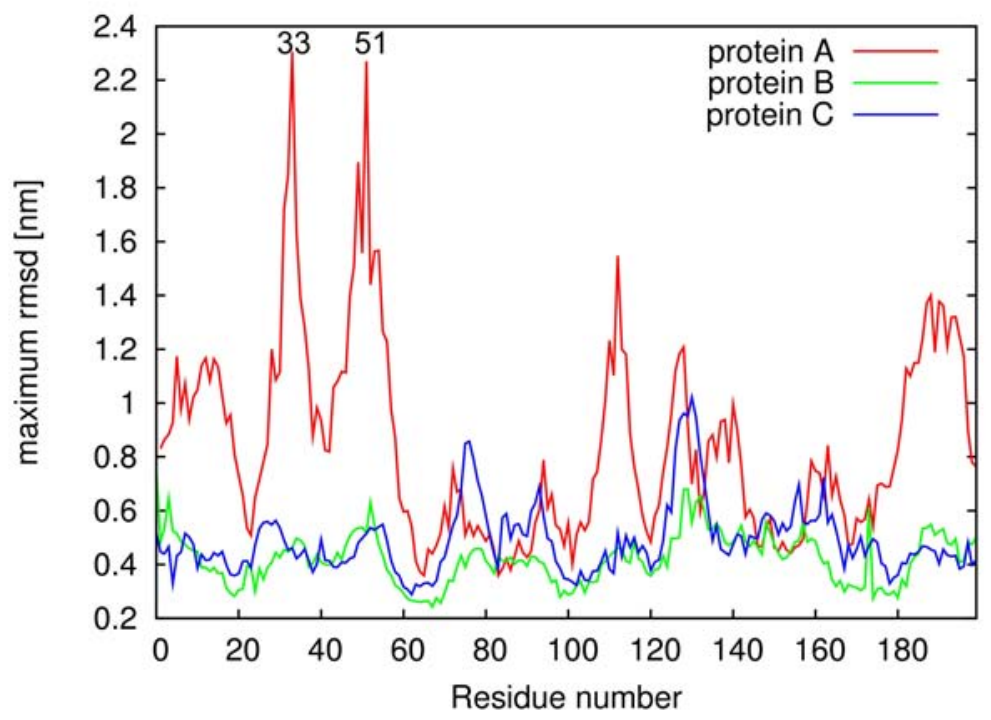

Figure 5.10: Largest rmsd for each residue of protein A (red), B (green), and C (blue) during the simulation in which the tip-sphere was moved through the center of protein A. The two largest peaks are identified by residue numbers.

The same observations were made when the tip-sphere was pushed along the 5 -fold symmetry axis through the center of the pentamer (Fig. 5.12). Here, the rmsd of the residues close to the pentamer center showed a high deviation from their initial position, whereas the overall pentamer structure remained stable during the simulation and was not deformed. 


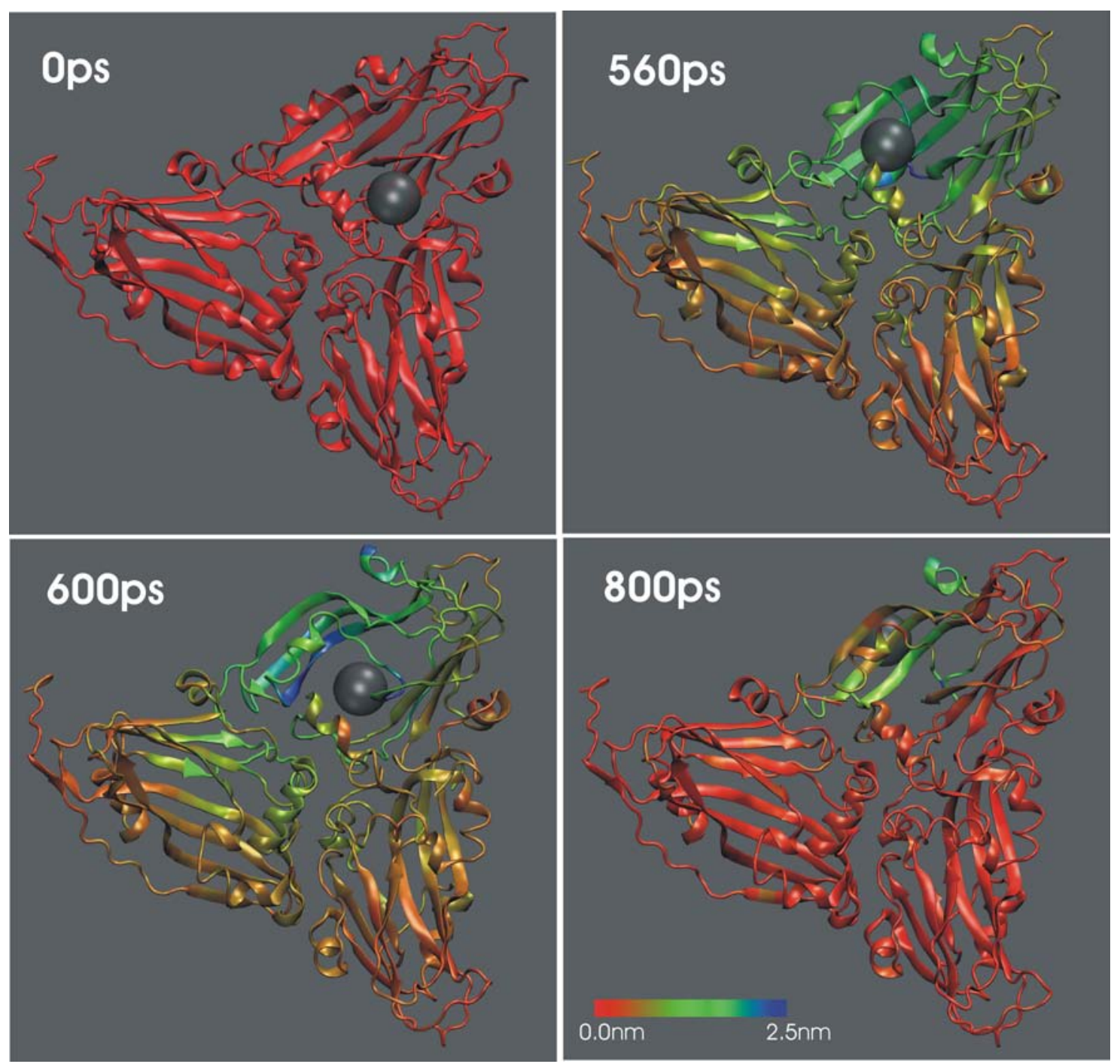

Figure 5.11: Subunit 12 during pushing the tip-sphere through the COM of protein A with color-coding for the deformation as in Fig. 5.6. 

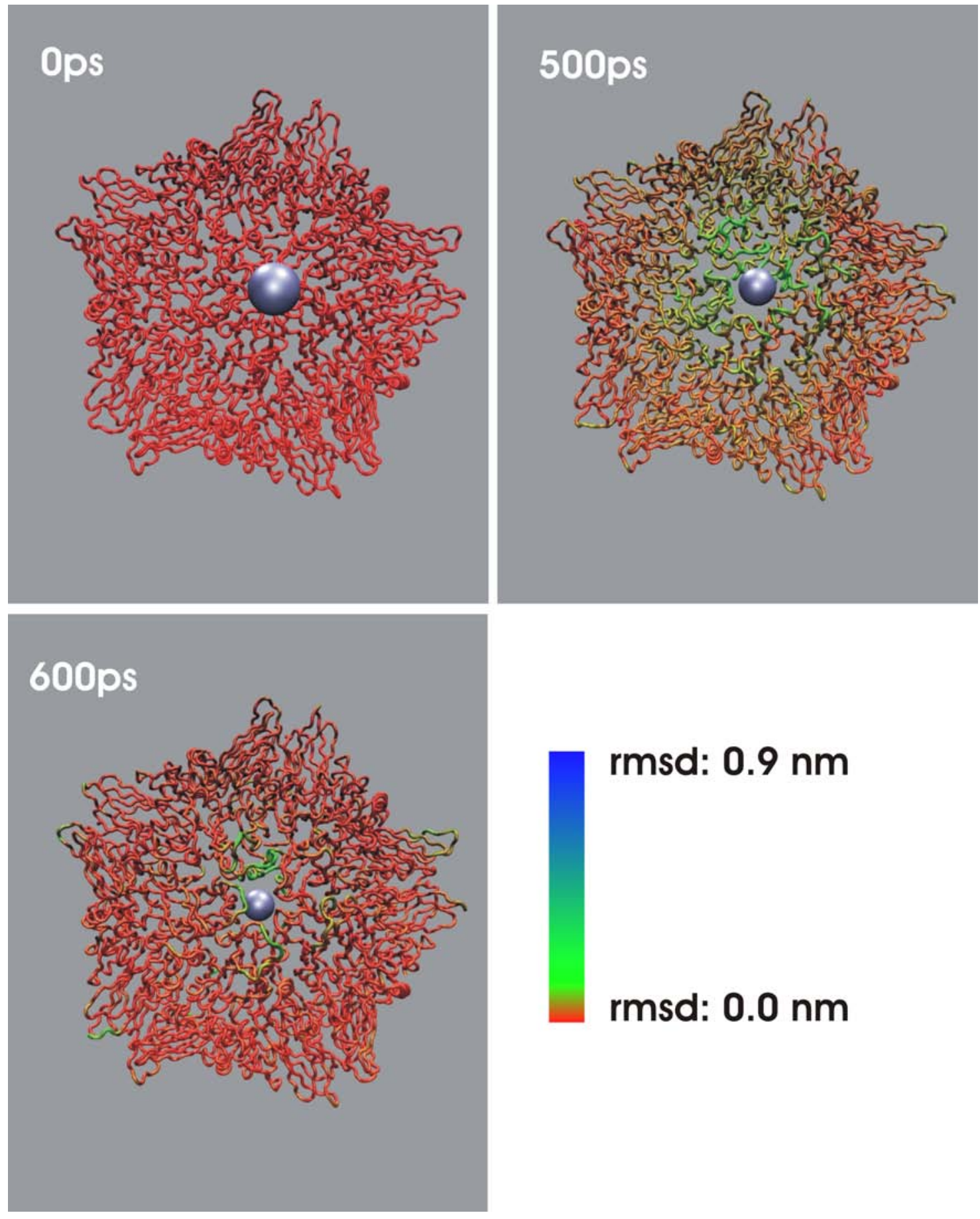

Figure 5.12: Pentamer consisting of five subunits. The tip-sphere (grey sphere) was pushed along the 5-fold axis through the pentamer center along the 5 -fold symmetry axis. Yielding was reached at 500 ps. Color-coding as in Fig. 5.6. 


\subsubsection{Cross-over from elastic to plastic behavior}

To address the nature of the deviation from linear to non-linear behavior within the rearrangement regime, we stopped several force probe simulations at different simulation times between $400 \mathrm{ps}$ and $600 \mathrm{ps}$ and subsequently let each of these systems relax for $0.5 \mathrm{~ns}$. In particular, we wanted to test if and when a plastic deformation of the few more strongly deformed residues identified above sets in. The tip-sphere was still kept inside the simulation box during relaxation, except that the external force onto the tip-sphere was switched off.
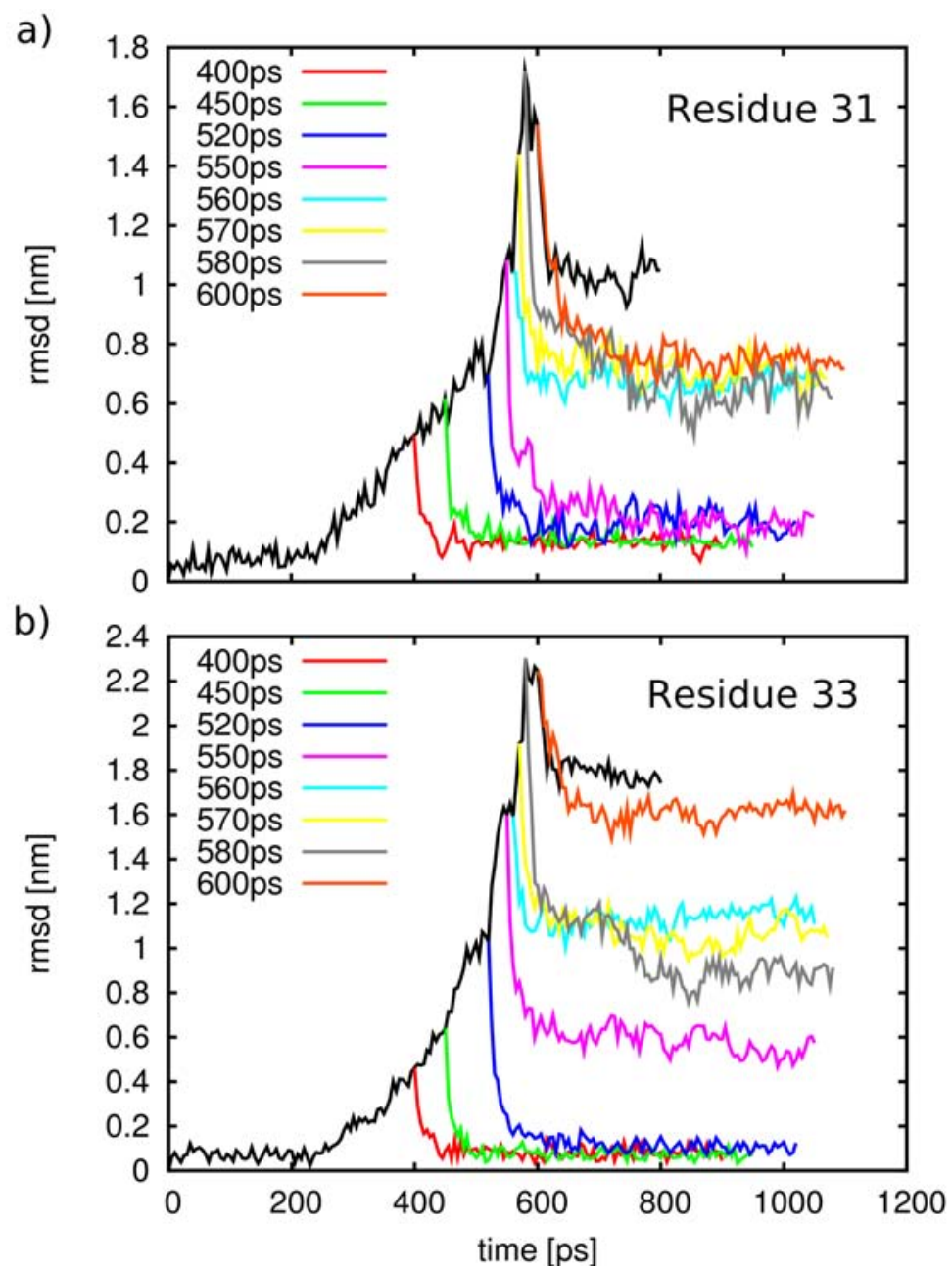

Figure 5.13: Rmsd (black curve) of residue 31 (a) and 33 (b) of protein A, SU 12. The FPMD simulation was stopped at different times (see legend) and restarted with unrestrained tip-sphere. During the subsequent relaxation processes, the rmsd of the two residues are shown in color. For residue 31, plastic deformation starts at $560 \mathrm{ps,} \mathrm{obtained} \mathrm{from} \mathrm{the} \mathrm{in-}$ creasing rmsd during the relaxation of the system. Residue 33 already behaves plastically at $550 \mathrm{ps}$. 
To characterize the deformation of the selected residues, Fig. 5.13 depicts the rmsd of the two residues, 31 and 33 (large values in Fig. 5.10, see also Fig. 5.16), fitted on the starting structure of the capsid.

The relaxation rmsd curves fall into two groups. When the force probe simulation was stopped at $550 \mathrm{ps}$ or earlier, the deformation of residue 31 and 33 was fully reversible and therefore elastic. In contrast, for switch-off times of $560 \mathrm{ps}$ or later, the rmsd dropped only partially and did not return to its original value, - at least within the 0.5 ns equilibration phase - , suggesting a plastic deformation of residue 31. A similar behavior is seen for residue 33, except that plastic behavior is already observed at $550 \mathrm{ps}$. Therefore, fully elastic behavior at the single residue level is found not only for the linear regime, but also essentially for the whole rearrangement regime.

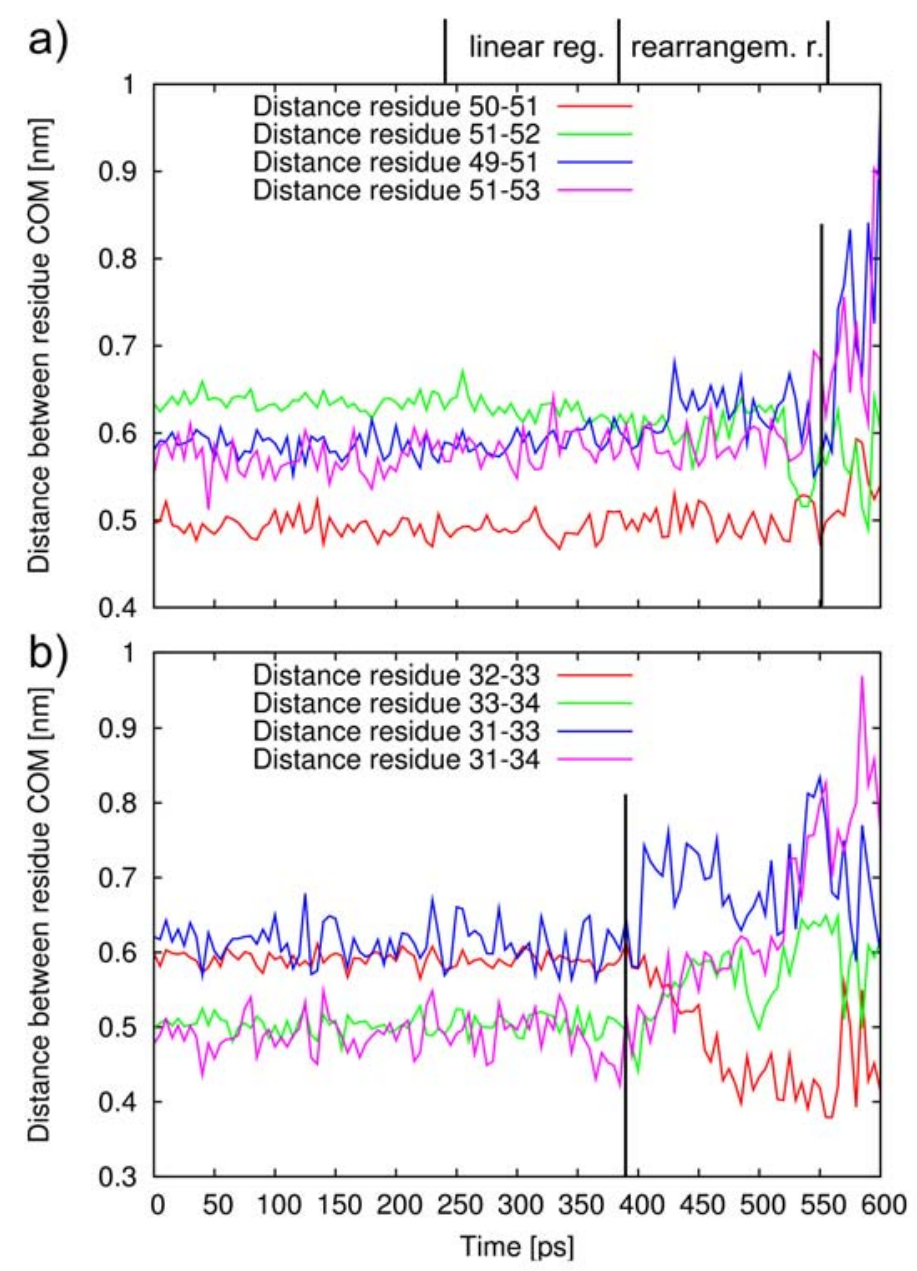

Figure 5.14: Distances between the center of mass (COM) of residue 51 (a) and residue 33 (b) to adjacent residues during deformation. The black vertical bars denote the yielding point (a) and the transition from the linear to the rearrangement regime (b). 
To further address the apparent differences between the two regimes and in particular the origin of the larger force fluctuations and sublinear behavior observed for the rearrangement regime, Fig. 5.14a,b shows the distances between the COM of residue 51 and 33 with their respective neighbors during the deformation.

As can be seen, within the linear regime, the distances between the nearest neighbors (residues 50-51, 51-52 and 32-33, 33-34) and next nearest neighbors (residues 49-51, 51-53 and 31-33, 31-34) remain constant within the range of thermal fluctuations $(<0.5 \AA$, Fig. 5.14). Here, in line with the collective elastic movement of the complete subunit observed already above, the elastic deformation of residues can be described as a displacement of the complete protein structure, whereas the overall shape and especially the residue distances are retained.

The distances between residue 51, an amino acid of the $\beta$-sheet region located behind residue 33 in Fig. 5.11 and 5.16, and its neighbors were conserved for approx. $550 \mathrm{ps}$ (Fig. 5.14a). Only for later times, when yielding occurred around $550 \mathrm{ps}$, the distances deviated, whereas the changes in next nearest neighbor distances dominated, e.g. residue 49 and 51, blue line in Fig. 5.14a. We suggest that the observed stiffness is characterized by a conservation of the topology of the $\beta$-sheet region during deformation. From these observations, the cross-over from elastic to plastic behavior is described by a change in residue neighbor distances. However, this result alone does not reveal the underlying mechanism of the rearrangement regime. To address this issue, we now focus on residue 33 and its neighbor distances as a function of time (Fig. 5.14b).

Interestingly, abrupt distance changes and fluctuations are seen starting already at the transition to the non-linear behavior of the rearrangement regime. Here, these changes in the neighbor distances describe markedly increased fluctuations of single atoms that were close to the tip-sphere during the simulations. Due to the interaction with the tip-sphere, these fluctuations translate into similarly increased force fluctuations in the force-distance curve as seen in Fig. 5.1, despite the fact that the deformation of the residues was still reversible.

We rationalize these findings by the simplified free energy landscape sketch shown in Fig. 5.15. For the whole linear regime, the system is confined to a local minimum of the underlying free energy landscape (Fig. 5.15, minimum 1), giving rise to a nearly linear force response. For further deformation, at the transition to the rearrangement regime, the deformation energy is large enough for the system to overcome the barrier to new local minima (2 and 3 in Fig. 5.15). As the energy of these new minima is larger than that of the initial minimum, and because thermal energy suffices to drive recrossings, frequent transitions between the minima result. As one of several possibilities, in this simple picture, the frequent transitions describe the origin of the elasticity as well as the larger fluctuations in the rearrangement regime. In particular, elastic deformation is described in terms of relaxation of the system to the initial minimum when external forces have been switched off. Note that this model can describe plastic deformations. In this case, return of the system to the original state is hampered by large energy barriers between the minima. 


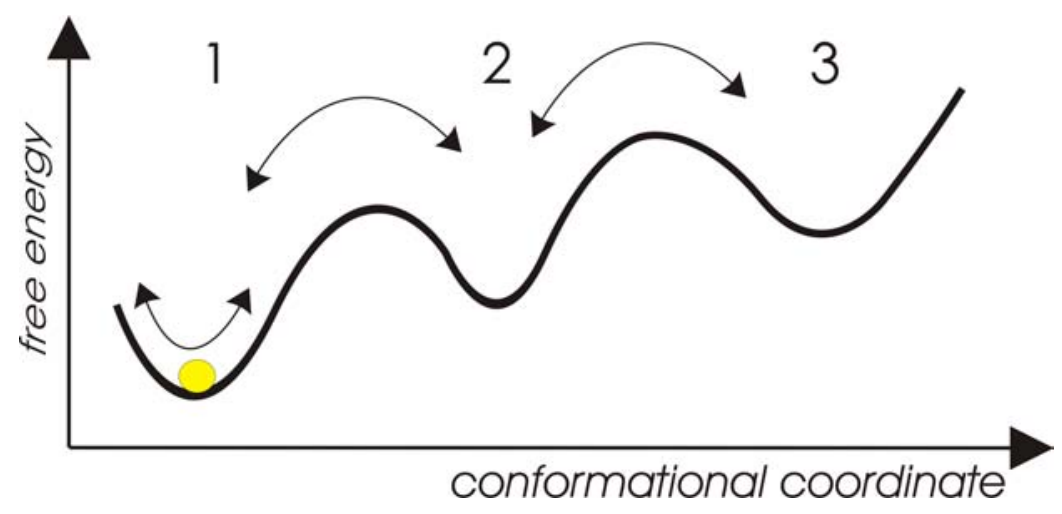

Figure 5.15: Simplified free energy landscape which can explain the large distance fluctuations apparent in Fig. 5.1 within the rearrangement regime, while the system is still able to quickly relax into the initial state. The small fluctuations in the linear regime reflect motions within minimum 1 (yellow); the large fluctuations arise from transitions between several distinct minima (arrows)

The cross-over from elastic to plastic deformation of residue 31 and 33 is observed when the distances between residue 32 and 33 decreased from $0.6 \mathrm{~nm}$ to $0.4 \mathrm{~nm}$, whereas the next nearest neighbor distances of residue 31 and 33 increased from $0.6 \mathrm{~nm}$ to $0.8 \mathrm{~nm}$ and those between residues 31 and 35 from $0.5 \mathrm{~nm}$ to $0.8 \mathrm{~nm}$ (Fig. $5.14 \mathrm{~b})$.

This shift in distances can be observed for several residues, e.g., for residues 39-42 which form an $\alpha$-helix (Fig. 5.16). A widening of the helix within less than $20 \mathrm{ps}$ defined the plastic yielding of the single helix residues, whereas the plastic deformation of residue 33 is determined by an isomerization of the aromatic ring at $560 \mathrm{ps}$. In summary, the rearrangement regime is characterized by a marked intramolecular structural change. The transition from elastic to plastic deformation occurs near the yielding point. We suggest that the larger force fluctuations in the force-distance plot compared to the linear regime are caused by a change from (nearly) harmonic dynamics to highly non-linear conformational transitions between multiple free energy minima. 

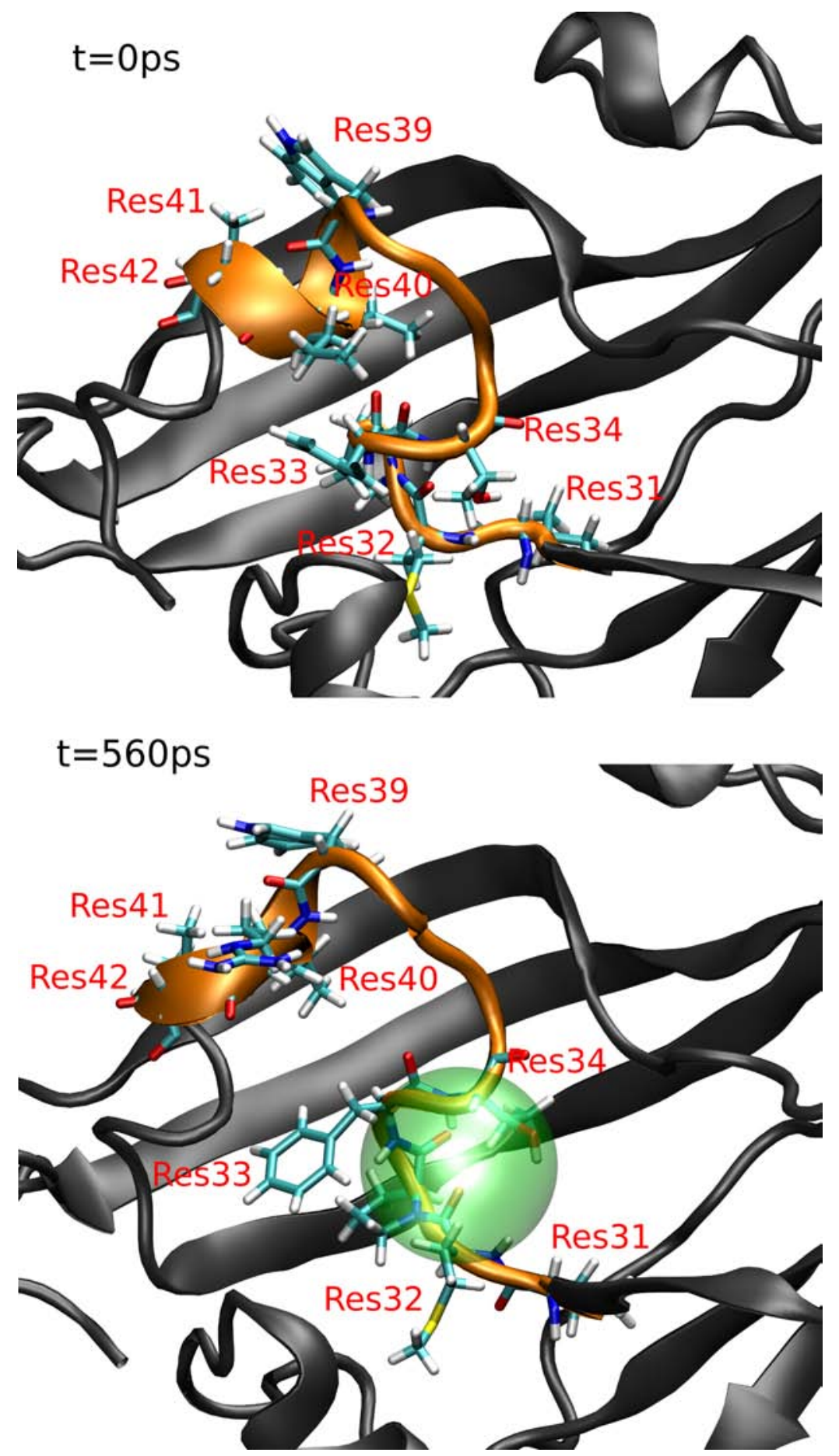

Figure 5.16: Detail of protein A, SU 12 before deformation (top) and during deformation with the tip-sphere (green) at the yielding point (560 ps, bottom). Relevant residues are shown as sticks. The $\alpha$-helix (orange ribbon, residue 39-42) is stretched, together with residues 31-34. The plastic deformation of residue 33 is characterized by an isomerization of the aromatic ring. 


\subsubsection{Influence of the tip-sphere size}

To study if the mechanical properties depend on the choice of the tip-sphere size, additional FPMD simulations with a larger tip-sphere were performed. In these simulations, the tip-sphere was pushed towards the center of the pentamer along the 5 -fold symmetry axis with a probe-velocity of $0.01 \mathrm{~nm} / \mathrm{ps}$ and an increased LennardJones parameter for the van-der-Waals radius of the tip-sphere, $\sigma=10.0 \mathrm{~nm}$. Subsequently, elastic constants and yielding forces were compared with previous results obtained for $\sigma=5.0 \mathrm{~nm}$.

For the larger tip-sphere, an elastic constant of $4.3 \mathrm{~N} / \mathrm{m}$ was obtained, the same within the error bars to the value obtained above for a smaller tip-sphere. In contrast, a markedly larger yielding force of $4.8 \mathrm{nN}$ was seen, compared to $3.0 \mathrm{nN}$ for the smaller tip-sphere. This result is not unexpected because, as can be seen from the simulations (Fig. 5.18, compare with Fig. 5.12), the regime of elastic deformation is roughly of the size of one pentamer (approx. $14 \mathrm{~nm}$ in diameter) and thus much larger than the two tip-spheres used. Therefore the elastic behavior does not depend on tip-sphere as long as the tip-sphere size is much smaller than the size of the subunit. In contrast, the regime which undergoes the more severe plastic deformation grows with the size of the penetrating tip-sphere, such that the yielding forces increase accordingly.

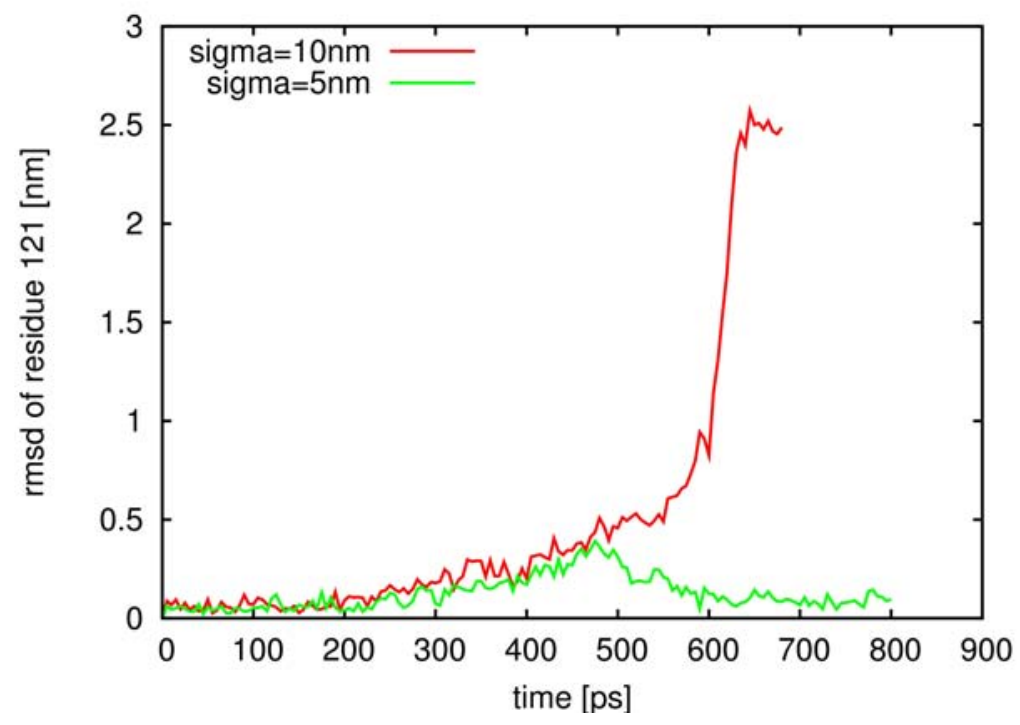

Figure 5.17: Rmsd of SU 12, residue 121, during indentation of the pentamer center, extracted from a simulation with the big, $\sigma=10 \mathrm{~nm}$ (red), and small, $\sigma=5 \mathrm{~nm}$ (green), tip-sphere. Yielding was reached at $490 \mathrm{ps}$ for the small tip-sphere and at $560 \mathrm{ps}$ for the big tip-sphere.

Furthermore, residues close to the indentation position of the tip-sphere deformed much stronger for the larger tip-sphere than for the small one (Fig. 5.17). After yielding at the maximum force at $550 \mathrm{ps}$, the tip-sphere left the shell towards the capsid center, and the surrounding residues "snapped away" and exhibited a large 
dislocation with respect to their initial positions. The larger deformations caused by the bigger tip-sphere resulted in a larger deformation energy that was stored in the single amino acids during indentation and was transformed into kinetic energy after the tip-sphere left the capsid (600-650 ps in Fig 5.17), resulting in a large rmsd of single residues. 

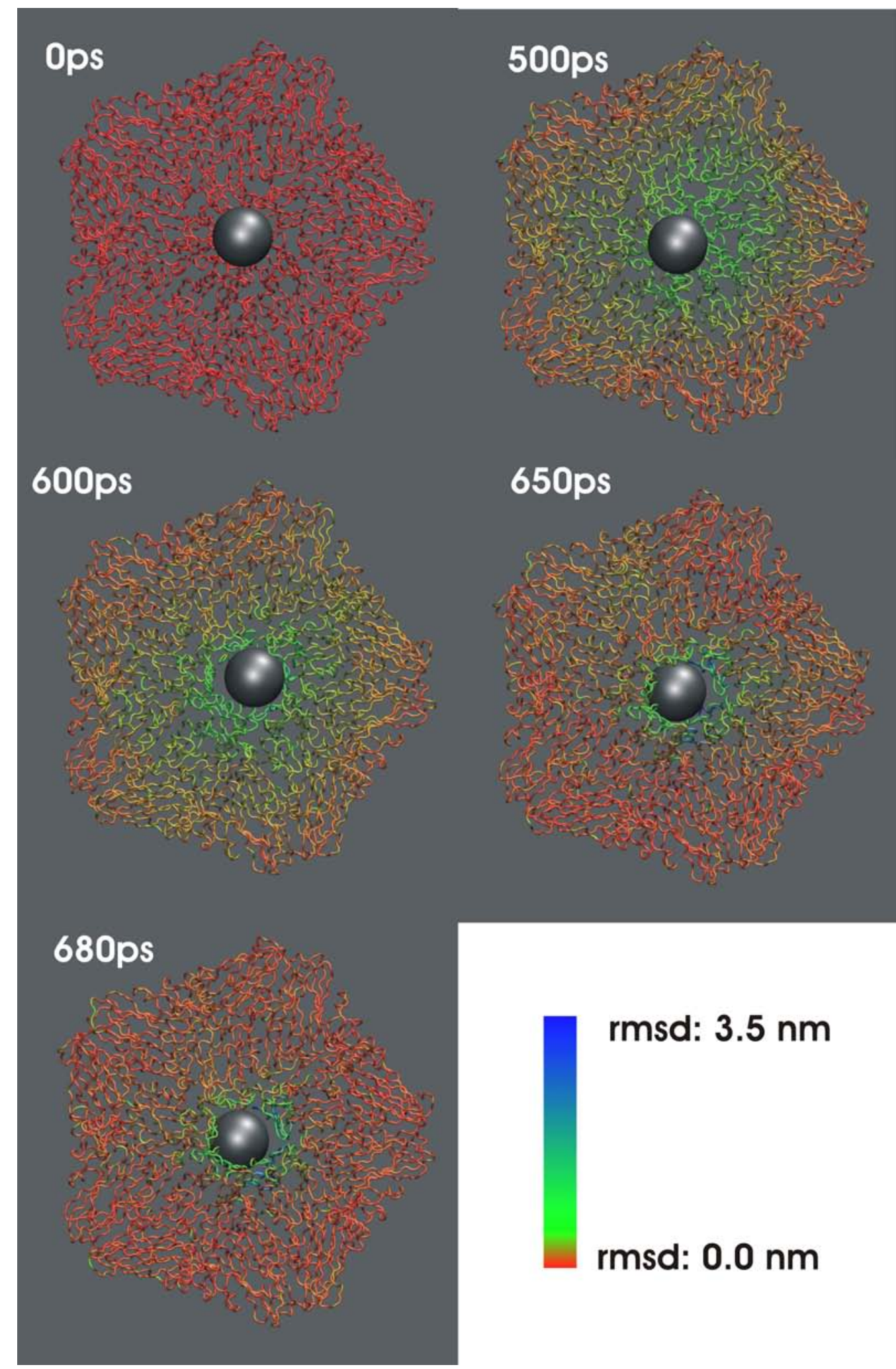

Figure 5.18: Pushing the tip-sphere (grey sphere, $\sigma=10 \mathrm{~nm}$ ) along the 5-fold axis through the center of a pentamer (tube representation). The deformation of each residue is color-coded as in Fig. 5.6. Although yielding was reached $560 \mathrm{ps}$, the largest deformations occurred between 600-650 ps, see also Fig. 5.17. 


\subsubsection{Influence of probe-velocity}

In order to study if and how the observed mechanical properties of the viral shell depend on probe-velocity, all FPMD simulations in which the tip-sphere was directed against the outer capsid surface with calcium ions in the structure were repeated with identical parameters, but with a five times larger probe-velocity of $0.05 \mathrm{~nm} / \mathrm{ps}$.
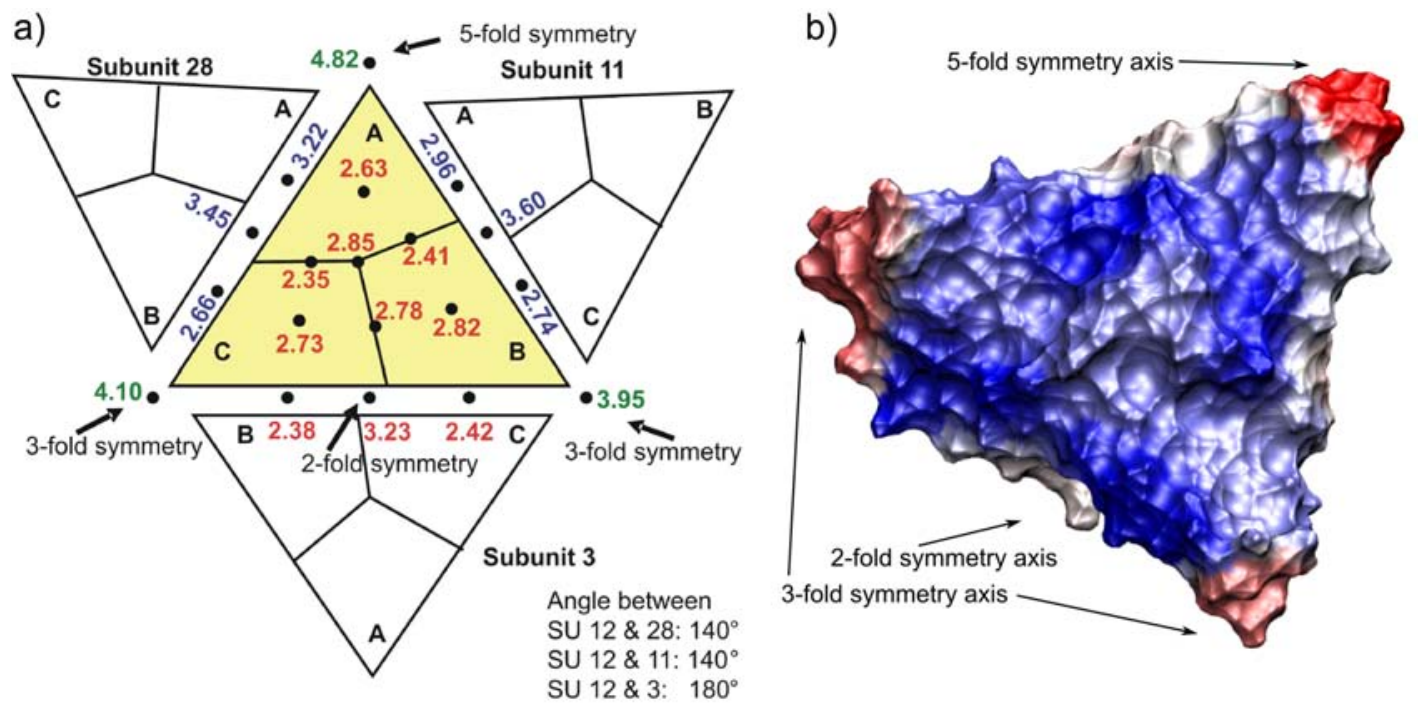

Figure 5.19: a) Elastic constants (in $\mathrm{N} / \mathrm{m}$ ) obtained for the capsid of SBMV with calcium ions as shown in Fig. 4.14. The tip-sphere was pushed with a probe-velocity of $0.05 \mathrm{~nm} / \mathrm{ps}$ against the outer surface of the shell. b) Color-coded distribution of elastic constants on subunit 12, obtained from values shown in a) (soft: blue, stiff: red).

A similar distribution of elastic constants as for the $0.01 \mathrm{~nm} / \mathrm{ps}$ simulations shown above was seen with the individual values consistently increased by about $14 \%$ (Fig. 5.19). The only exceptions were for the elastic constants obtained at the subunit center which exhibited a larger increase from $2.16 \mathrm{~N} / \mathrm{m}$ to $2.85 \mathrm{~N} / \mathrm{m}$ and the 2 -fold axis, where the value increased from $1.89 \mathrm{~N} / \mathrm{m}$ to $3.23 \mathrm{~N} / \mathrm{m}$. We attribute this strong velocity dependence to the particularly large flexibility observed for the residues at the subunit center and the 2-fold symmetry axis, which was mostly reduced at larger probe velocities. Also the yielding forces showed a consistent increase by about $33 \%$ for larger probe-velocities with the overall distribution of $F_{\max }$ remaining constant (Fig. 5.20).

To more quantitatively characterize the dependence of the mechanical properties on the probe-velocity of the tip-sphere, force-probe simulations towards the center of a pentamer along the 5-fold symmetry axis were performed with nine different probevelocities between $0.001 \mathrm{~nm} / \mathrm{ps}$ and $0.05 \mathrm{~nm} / \mathrm{ps}$. For each of the nine velocities, five simulations were carried out and averaged (Fig. 5.21). 
a)

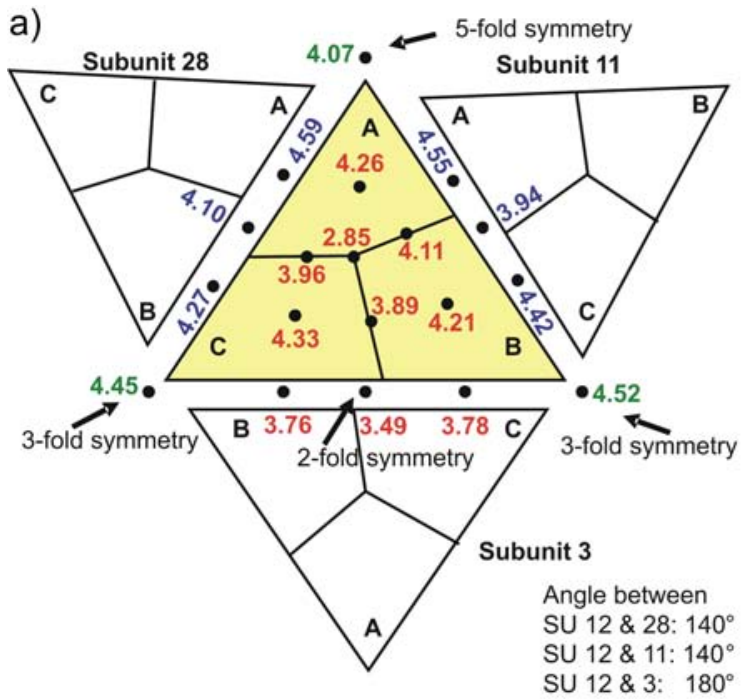

b)

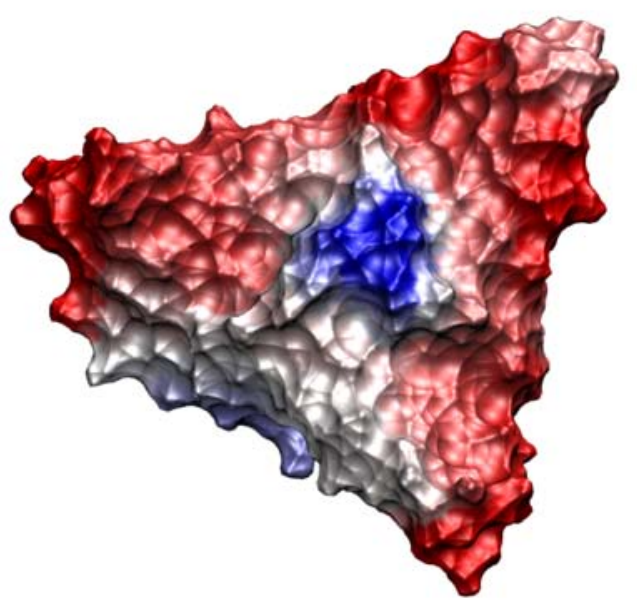

Figure 5.20: Obtained yielding forces (in $\mathrm{nN}$ ) shown as in Fig. 5.19 for a probevelocity of $0.05 \mathrm{~nm} / \mathrm{ps}$ (blue: low stability, red: high stability).

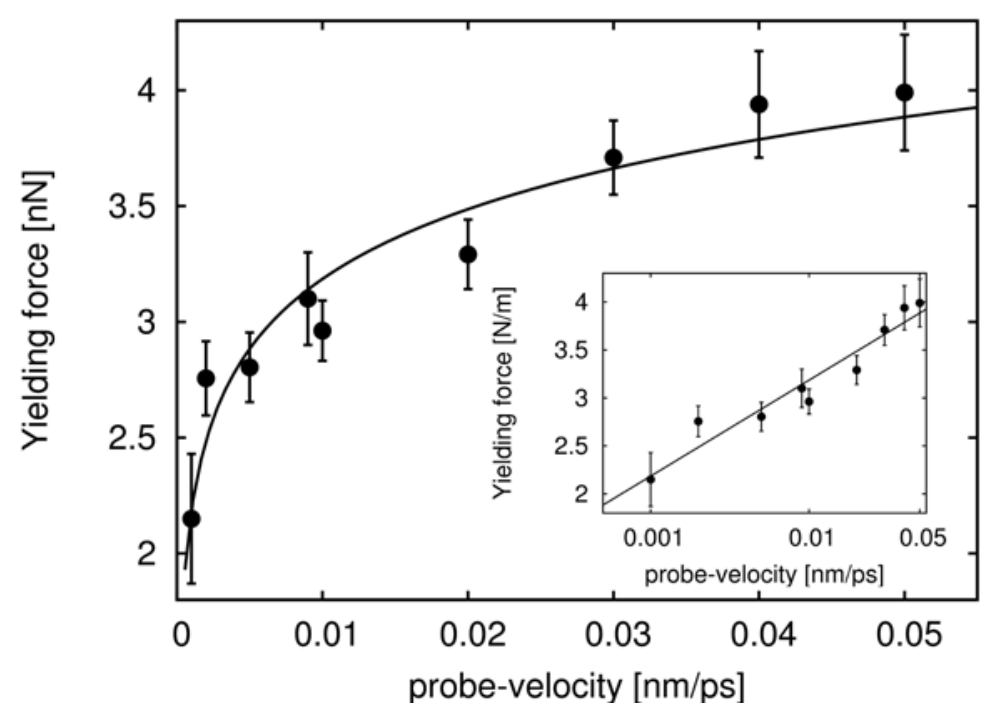

Figure 5.21: Yielding forces for different probe-velocities, where the tip-sphere was directed towards the center of the pentamer along the 5-fold symmetry axis. The dots and error bars denote the averages and its errors estimated from the variances from five simulations each. Logarithmic fits, $f(x)=a+b \cdot \log \left(\frac{x}{x_{0}}\right)$, are shown as lines. The inset shows the same data on a logarithmic velocity scale. 
As can be seen, the yielding forces depend logarithmically on probe-velocity over nearly two decades (Fig. 5.21). This observation is supported by the good agreement with the logarithmic fit (solid line), as well as by the inset, which shows the same data on a logarithmic velocity scale. Such logarithmic behavior is well known and has been observed previously for many macromolecular systems, such as ligand unbinding [138] or protein unfolding [52, 139]. This behavior is most easily described by activated barrier crossing following Kramers theory [140, 141], or by more refined theories [142-146]. All these treatments rely on the Kramers assumption that all degrees of freedom perpendicular to the reaction coordinate are at or close to equilibrium during the (non-equilibrium) unbinding, unfolding, or deformation process, i.e., that this process is governed by an underlying time-independent one-dimensional free energy landscape. One prediction of this treatment is that the variation of the yielding force with probe-velocity is due to the variation of the point where thermal activation causes rupture with probe-velocity. A second property is that - due to the unchanged energy landscape - the initial response of the system at low forces is independent of the probe-velocity (possibly only affected by frictional forces, which typically show a linear dependence).

For the case at hand, this initial response is probed by the elastic constants (Fig. 5.22). As can be seen, however, also those show a clear logarithmic behavior, contrary to the above expectation. Further, the indentation point where fracture occurs does not markedly vary with probe-velocity. Apparently, simple Kramers-like models are incompatible with our results.

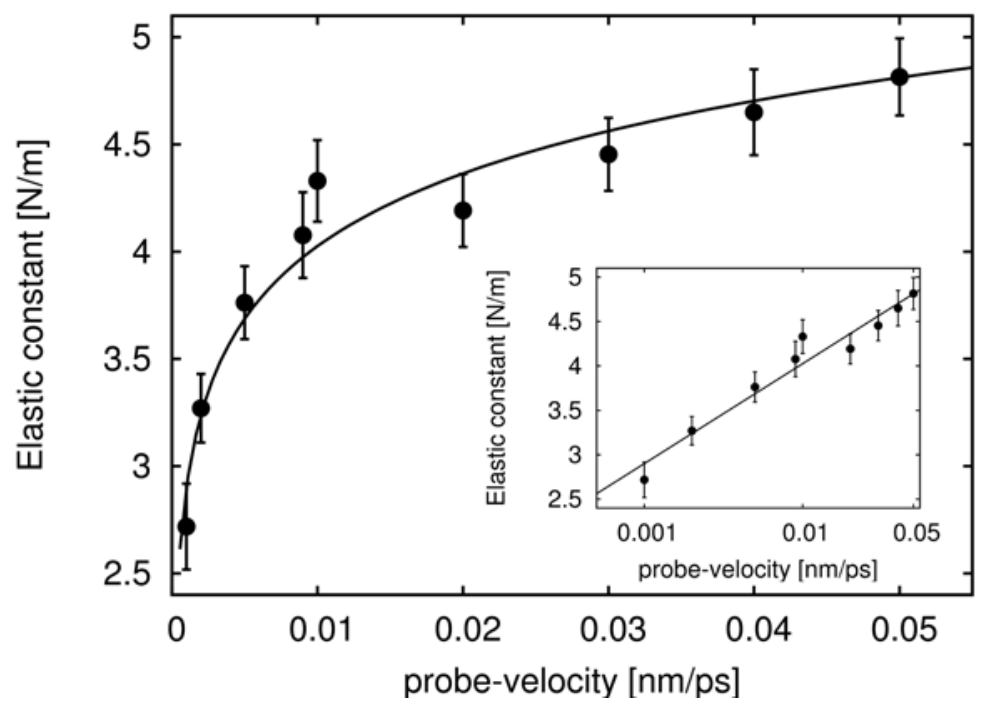

Figure 5.22: Elastic constants obtained for different probe-velocities as described in Fig. 5.21.

These discrepancies force us to extend the theory of enforced barrier crossing. Particularly striking here is the very similar rate dependence of the yielding forces and 
the elastic constants, which suggests a common cause. We therefore tentatively attribute this behavior to slow relaxation effects of degrees of freedom perpendicular to the reaction coordinate. Because the linear (elastic) response of the applied force in the force-time and force-distance curve (Fig. 5.1) implies that the overall shape of the underlying energy landscape is close to harmonic in the vicinity of the minimum, we suggest that relaxation motions, e.g. within or between amino acids in contact with the tip-sphere imply a time-dependence of the effective free energy landscape governing the forces that act on the tip-sphere. As a result, the height of the barrier opposing penetration is assumed to gradually decrease in the process, which, generalizing Bell's theory, translates into the observed rate dependence. Accordingly, for slower probe-velocities, the barrier height is lowered to a larger extent, whereas its position remains nearly unchanged. Because a similar effect can be expected for the curvature of the minimum such that it scales synchronously to the barrier height, the common behavior of the elastic constant and the yielding force follows as a natural consequence from our theory.

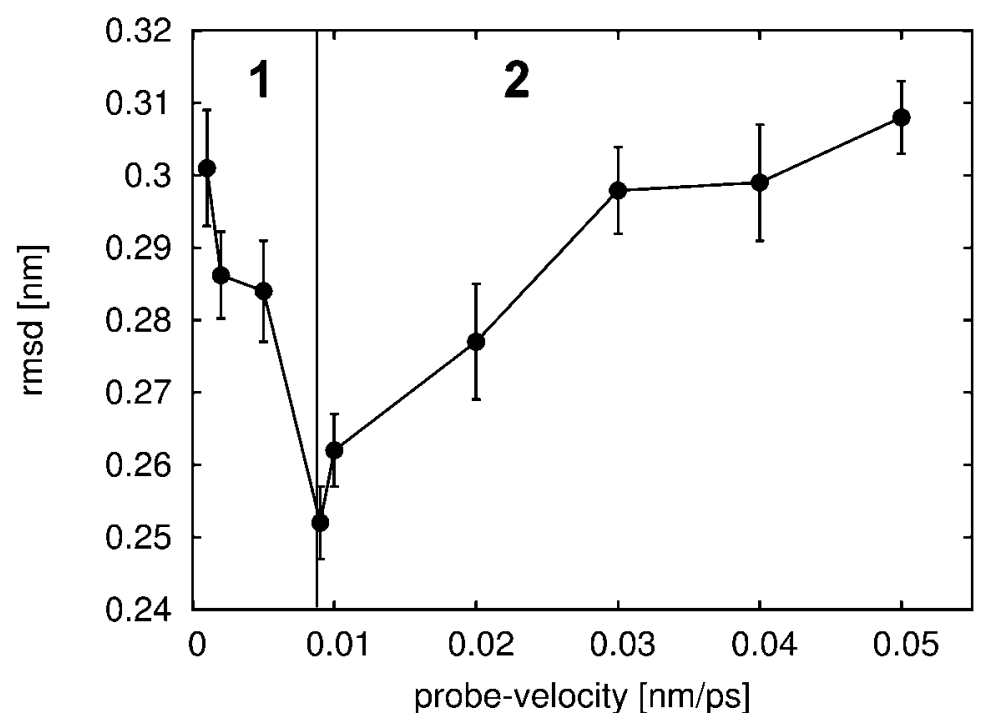

Figure 5.23: Maximum rmsd values of protein A residues close to the 5-fold symmetry axis for different probe-velocities. Two regimes are found, (1) and (2) as explained in the text.

If the proposed relaxation actually occurs, this relaxation should be visible also in the dynamics of the capsid close to the tip-sphere during indentation. In particular, for slower probe-velocities and the associated larger time-scales, the relaxation process will follow the perturbation induced by the tip-sphere to a larger extent. Accordingly, larger structural rearrangements are expected. We therefore calculated the rmsd of the residues close to the 5-fold symmetry axis during deformation with respect to the initial structure of the capsid. Indeed, an increasing rmsd for decreasing probe-velocity is observed (Fig. 5.23, regime 1), thus, proposing our model as 
a valid description of the observed logarithmic behavior of both yielding forces and elastic constants.

The smallest rmsd in Fig. 5.23 and the cross-over from regime 1 and 2 is found for a probe-velocity of $0.009 \mathrm{~nm} / \mathrm{ps}$. The behavior in regime 2 in Fig. 5.23 is not determined by relaxation dynamics of single residues close to the tip-sphere, but competing spring constants of the tip-sphere and the protein at the capsid position the tip-sphere is pushed through. For these high probe-velocities, the capsid proteins behave stiff (high spring constant) when the tip-sphere is pushed against the capsid. The corresponding residues close to the tip-sphere are "kicked" away from the tipsphere resulting in an acceleration of the single residues; here relaxation dynamics are hampered. These accelerations are reduced for slower probe-velocities in regime 2 , because the kinetic energy transferred from the tip-sphere to the residues is reduced for slower tip-sphere motions, and the maximum rmsd values of the respective residues are lowered.

\subsubsection{Influence of probe-velocity - Monte Carlo simulations}

In order to validate our assumptions described above that relaxation dynamics perpendicular to the deformation coordinate can result in the observed velocity dependence of elastic constants and yielding forces, we performed Monte Carlo (MC) simulations. MC simulation is a numerical method which uses repeated random sampling to calculate probability distributions of various processes in science, economics and evolution. The MC simulation applied here numerically calculates the diffusion of a particle in a harmonic potential with an external force acting on the particle. Here relaxation dynamics perpendicular to the reaction coordinate are possible (Fig. 5.24). The one-dimensional case, i.e., a harmonic energy landscape $G_{0}(x)=\frac{1}{2} k_{0} x^{2}$ with a sharp maximum at $x=L$ as shown in Fig. 5.24 and a force acting in $\mathrm{x}$ direction results in velocity independent elastic constants and a logarithmic rupture force - velocity dependence, as already studied in detail [141, 144]. 


\section{Methods}

For the case at hand, the diffusion of a system (particle) in a two-dimensional harmonic energy landscape is considered for our MC simulations,

$$
G(x, y)=\frac{1}{2} k_{\perp}(x-\alpha y)^{2}+\underbrace{\frac{1}{2} k_{0} x^{2}}_{G_{0}(x)}-\frac{1}{2} k_{\perp} x^{2}
$$

with $x=x(t)$ and $y=y(t) . \quad k_{0}$ is the spring constant of the one-dimensional harmonic energy landscape $G_{0}(x)$, the spring constant $k_{\perp}$ determines the diffusion and relaxation dynamics of the system along the relaxation coordinate $\alpha y$ with $\alpha$ being a positive number that gives the slope of the relaxation coordinate (Fig. 5.24). For a particle which is placed in the energy minimum of $G_{0}(\mathrm{x}), G(x, y)$ translates into relaxation dynamics along the relaxation coordinate $\alpha y$ and the diffusion pathway along $\left(\begin{array}{c}x \\ x / \alpha\end{array}\right)$ during the diffusion time $t$.

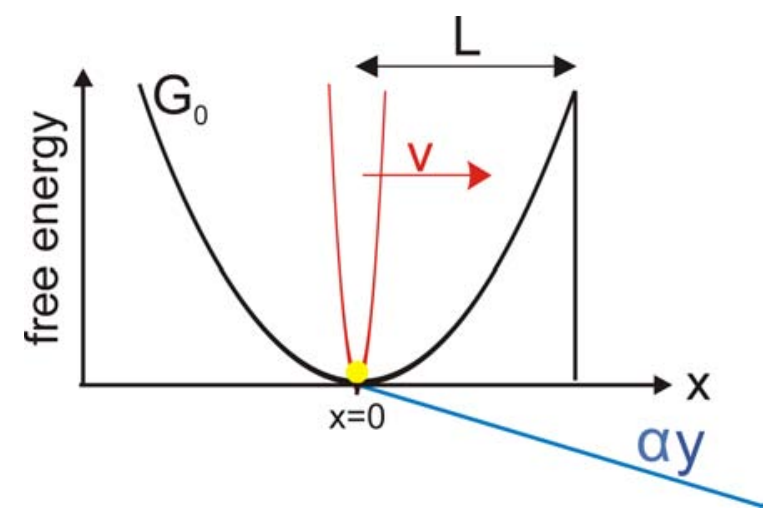

Figure 5.24: Sketch of energy landscape (black curve). The red line denotes the pull-potential which is moved with constant velocity $v$ is x-direction. $L$ marks the rupture length and $\alpha y$ the relaxation coordinate perpendicular to the reaction coordinate.

Additionally, a force acting on the system is applied, here characterized by a sharp harmonic potential (Fig. 5.24, red),

$$
V_{\text {pull }}(x, t)=\frac{1}{2} k_{\text {pull }}(x-v \cdot \Delta t)^{2}
$$

which moves into positive $x$ direction with constant velocity $v$ during a time step $\Delta t ; k_{\text {pull }}$ is the spring constant of this pull-potential. In order to compare the MC simulation results with the force-probe simulations, a rupture length $L$ is included within the potential $G_{0}(x)$ (Fig. 5.24). When the particle is pulled along the reaction coordinate, the energy drops to 0 when $x=L$ is reached, describing a barrier crossing which, with respect to the deformation behavior of the viral shells, models fracture. 
As a start, a particle was placed in the potential minimum of $G_{0}(x)$ at $x=0$, i.e. $G(0, y)=\frac{1}{2} k_{\perp}(-\alpha y)^{2}$ with $V_{\text {pull }}(x, t)$ acting on the system. In order to derive the particle position after the first MC step $-x_{n e w}$ and $y_{\text {new }}-$ a Gaussian distribution around the particle position at $x_{0}$ and $y_{0}$ was calculated with the Gaussian width $\sigma$ determined by the chosen step size. A possible particle position within such Gaussian distributions in $\mathrm{x}$ - and $\mathrm{y}$-direction was then selected by a random number generator. The probability distributions for the displacements $\Delta x$ and $\Delta y$ of the particle in $\mathrm{x}$ and $\mathrm{y}$-direction during one MC step are given by

$$
\begin{aligned}
& p(\Delta x)=\frac{1}{\sigma \sqrt{2 \pi}} \exp \left(-\frac{1}{2} \frac{\Delta x^{2}}{2 \cdot \sigma^{2}}\right) \\
& p(\Delta y)=\frac{1}{\sigma \sqrt{2 \pi}} \exp \left(-\frac{1}{2} \frac{\Delta y^{2}}{2 \cdot \sigma^{2}}\right)
\end{aligned}
$$

The new particle position is given by

$$
\begin{aligned}
& x_{\text {new }}=x_{0}+\Delta x \\
& y_{\text {new }}=y_{0}+\Delta y
\end{aligned}
$$

Subsequently, the system energy $G\left(x_{n e w}, y_{n e w}\right)$ for the new coordinates was calculated from eq. 5.1. If $\Delta G=G\left(x_{n e w}, y_{\text {new }}\right)-G(x, y)<0$ the new system position was accepted. Otherwise the following Monte Carlo criterion had to be fulfilled for acceptance

$$
x_{i}<\exp (-\Delta G)
$$

with $x_{i}$ being a uniform distributed random number between 0 and 1 . If none of the two MC criteria was fulfilled, the step was neglected and new coordinates were calculated. The acceptance/rejection ratio depends on the step size; a ratio of $95 \%$ turned out to be a reasonable value which was obtained for a step size of $\sigma=0.001 \mathrm{~nm}$. In order to obtain a diffusion pathway of the particle, several thousand MC simulation steps were performed.

Several different MC simulation parameters for the step size, spring constants, etc., were used. It turned out that, with the following set of parameters, good agreement was obtained with the force-probe simulation results: $\Delta t=0.001 \mathrm{ps}$, $k_{0}=400 \mathrm{~kJ} /\left(\mathrm{mol} \cdot \mathrm{nm}^{2}\right), k_{\perp}=200 \mathrm{~kJ} /\left(\mathrm{mol} \cdot \mathrm{nm}^{2}\right), k_{\text {pull }}=1000 \mathrm{~kJ} /\left(\mathrm{mol} \cdot \mathrm{nm}^{2}\right), \alpha=$ $0.5, L=10 \mathrm{~nm}$. 15 different velocities were applied between $1 \cdot 10^{-8} \mathrm{~nm} / \mathrm{ps}$ and $1 \cdot 10^{-3} \mathrm{~nm} / \mathrm{ps}$. For each velocity a series of $8 \mathrm{MC}$ simulations was performed. The error was determined from the standard deviations at the averages.

From the force acting on the particle, $F(x)=k_{\text {pull }}(x-v \cdot \Delta t)$, and the distance the particle moved in $\mathrm{x}$-direction, a force-distance plot was obtained. From the linear regime in this curve, the elastic constant of the system was determined. The maximum force before the force dropped due to a barrier crossing, determined the yielding force. 


\section{Simulation results and discussion}

The elastic constant of the particle located in a two-dimensional harmonic potential, as well as the yielding force, were obtained from each MC simulation. A typical forcedistance plot is given in Fig. 5.25. A linear force increase was seen until $x=10 \mathrm{~nm}$, the rupture length $L$, with a subsequent force drop.

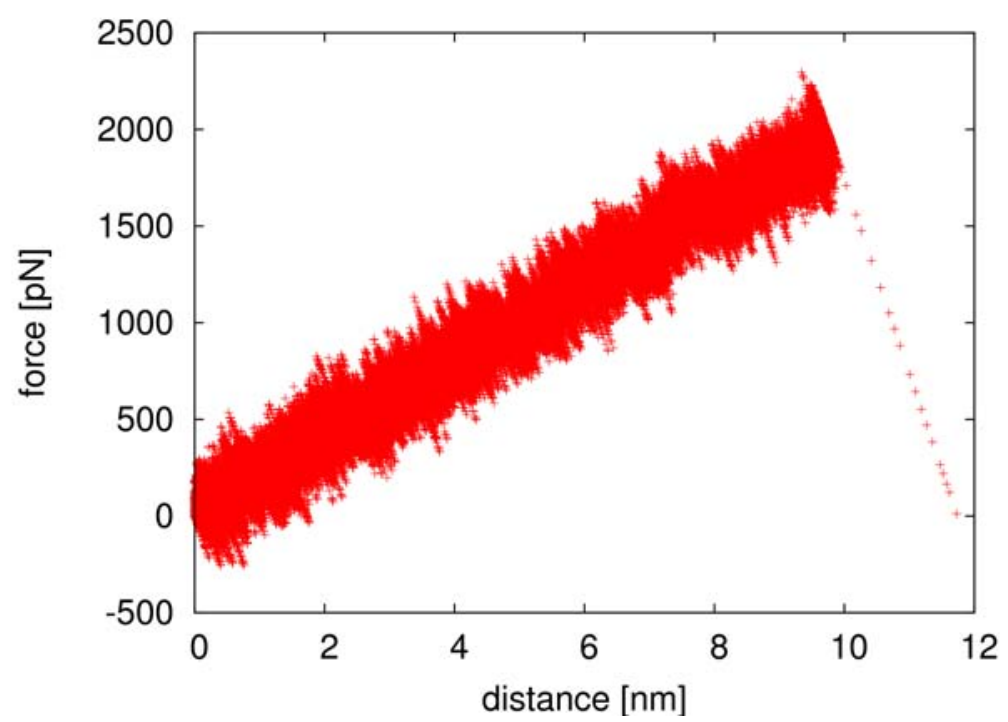

Figure 5.25: Force-distance curve for pull-velocity $1 \cdot 10^{-7} \mathrm{~nm} / \mathrm{ps}$.

Fig. 5.26 shows the obtained elastic constants and yielding forces as a function of pull-velocity on a logarithmic velocity scale. A clear logarithmic behavior can be seen for both plots for velocities $>5 \cdot 10^{-5} \mathrm{~nm} / \mathrm{ps}$, which is in good agreement with the logarithmic velocity dependence obtained from our force-probe simulations with probe-velocities in the range of $0.001-0.05 \mathrm{~nm} / \mathrm{ps}$.

For a velocity of $0.001 \mathrm{~nm} / \mathrm{ps}$, an elastic constant of $0.46 \mathrm{~N} / \mathrm{m}$ was seen in the MC simulations, nearly six times smaller than the value obtained from the force-probe simulations. The obtained yielding force from the $\mathrm{MC}$ simulations $(7.10 \mathrm{nN})$ was about three times larger than the force seen in the force-probe simulations. Therefore, although such a simplistic model was employed to corroborate our force-probe simulation results, the MC simulations reflect the mechanical properties of the system during deformation and the logarithmic velocity dependence very well. The obtained elastic constants and yielding forces deviate from the force-probe simulation results by less than one order of magnitude.

Thus, the MC simulations corroborate our proposed non-equilibrium effects within the established theory of enforced barrier crossing as a valid model for velocity dependent elastic constants and yielding forces.

For smaller velocities $<5 \cdot 10^{-5} \mathrm{~nm} / \mathrm{ps}$, the elastic constants and yielding forces leveled off at constant values because the system was always close or in equilibrium 

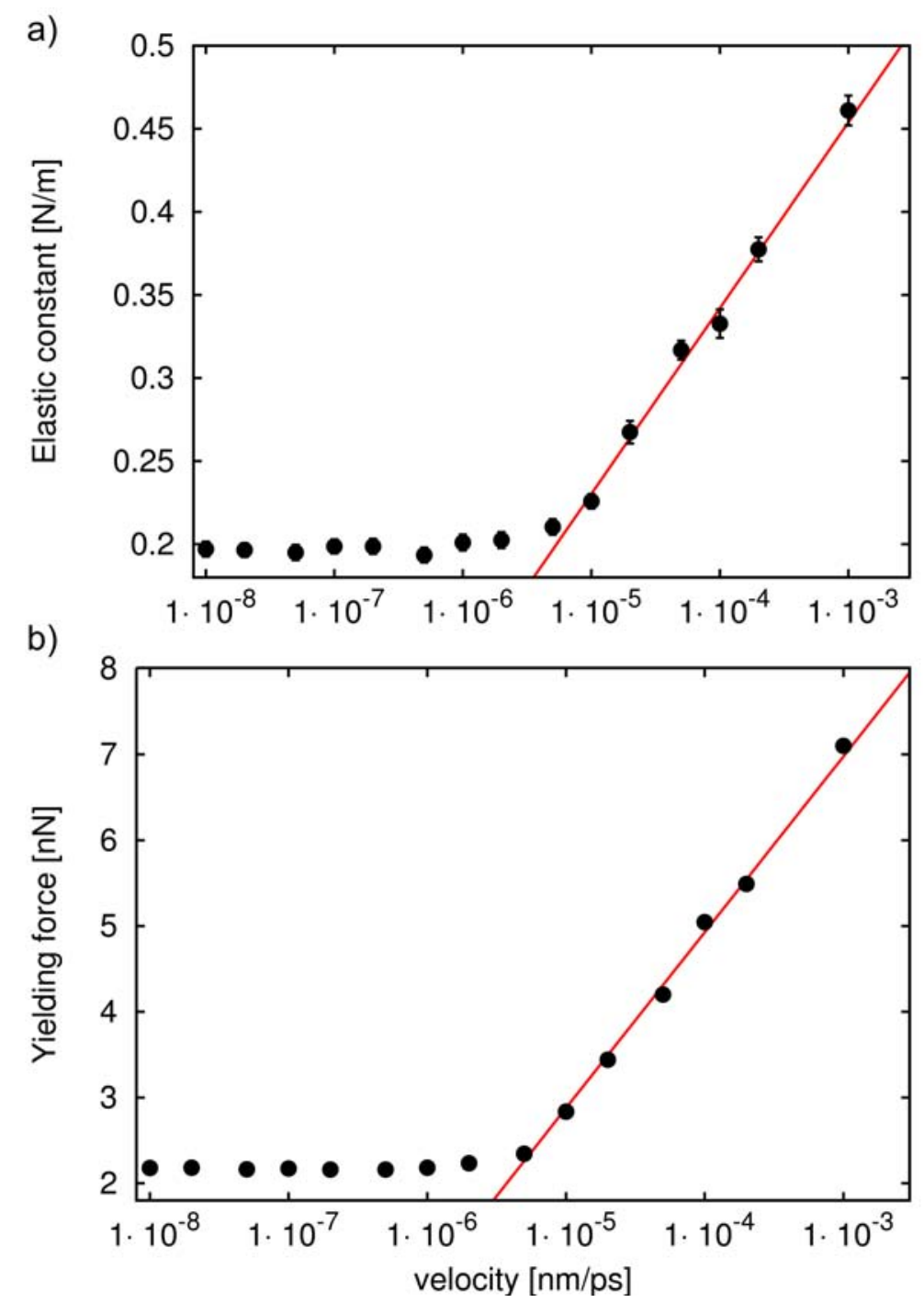

Figure 5.26: Velocity dependence of elastic constant (a) and yielding forces (b) from Monte Carlo simulations. Logarithmic fits, $f(x)=a+b \cdot \log \left(\frac{x}{x_{0}}\right)$, are shown as red lines. The errors for the yielding forces are about $0.03 \mathrm{nN}$, therefore the error bars are very small and not plotted.

along $\left(\begin{array}{c}x \\ x / \alpha\end{array}\right)$ on time scales of the applied deformation (Fig. 5.27). This crossover from a velocity dependent to an independent elastic constant describes the transition from our expanded model of enforced barrier crossings to Kramers theory in which the degrees of freedom perpendicular to the reaction coordinate are always in or close to equilibrium. In AFM experiments on viral shells, velocity independent elastic constants in the range of $0.2-0.6 \mathrm{~N} / \mathrm{m}$ were measured [31], similar to the obtained value from the MC simulations $(0.2 \mathrm{~N} / \mathrm{m})$ for velocities $<5 \cdot 10^{-5} \mathrm{~nm} / \mathrm{ps}$. These AFM experiments showed that in the Kramers-like regime the rupture length 


\subsection{Results and Discussions}

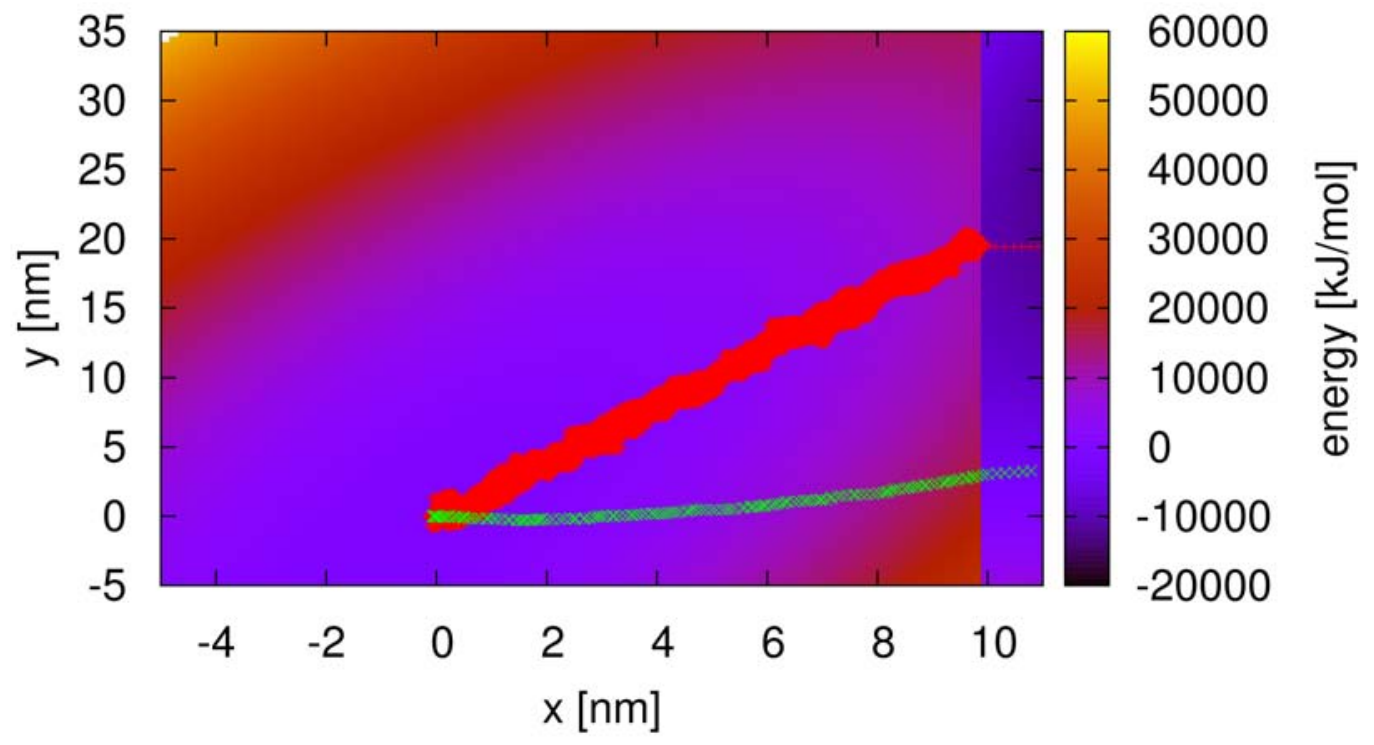

Figure 5.27: Color-coded energy landscape as described by eq. 5.1. The respective values for the energy are given by the color-bar. The diffusion pathway of the particle in the system which is pulled in positive $\mathrm{x}$ direction, is shown in red for a velocity of $1 \cdot 10^{-4} \mathrm{~nm} / \mathrm{ps}$ and in green for $1 \cdot 10^{-7} \mathrm{~nm} / \mathrm{ps}$. At the beginning of each simulation, the particle was placed in the energy landscape at $x=0 \mathrm{~nm}$ and $y=0 \mathrm{~nm}$.

$L$ depends on the deformation velocity, i.e. the rupture length increases for high velocities. Such a behavior translates into a logarithmic velocity dependence of the obtained yielding forces. In our MC simulation approach, the rupture length did not change with varying deformation velocities, and thus no velocity dependent yielding forces were determined.

To conclude, our MC simulations reflect the logarithmic velocity dependence of elastic constants and yielding forces found in the force-probe simulations very well. The transition to velocity independent mechanical properties for slower deformation velocities corroborated our assumptions that high probe-velocities cause relaxation dynamics perpendicular to the velocity vector, whereas for slower velocities these degrees of freedom are always in equilibrium. 


\subsection{Conclusions}

In this chapter, the structural changes of the shell proteins of Southern Bean Mosaic Virus during deformation were analyzed, as well as possible contributions to the observed forces due to friction and surface effects. Our investigations focus on the force-probe simulations, in which the tip-sphere was pushed from outside against and through the center of mass of protein A, subunit 12 with calcium ions in the structure. These simulations, together with the indentation results obtained for the other grip point positions on the inner and outer capsid show that the viral shell behaves highly elastic during indentation with the tip-sphere. Three different deformation regimes were identified in the force-distance and force-time behavior. First, a pre-linear regime was seen, resulting from local rearrangements of the outer surface layers when the tip-sphere approached the capsid. Such rearrangements were already suggested from elastic network normal mode analysis on SBMV and other capsids [26]. Second, a linear force increase was observed, which is characteristic for elastic deformation, and, third, a rearrangement regime of sub-linear force increase that yielded a maximum force before rupture occurred.

During deformation of the capsid, the water flux through the viral shell, as well as changes of the solvent accessible hydrophobic surface (SAS) area, were monitored. When the tip-sphere approached the capsid from outside, no water flux through the shell was observed, resulting in a compression of the water inside the protein shell. From the volume decrease inside the shell, an internal pressure increase to 5.43 bar was observed, thus causing a force acting from inside against subunit 12 of $22.8 \mathrm{pN}$, approximately $1 \%$ of the yielding force. An increase of the SAS area was only seen for deformations near the yielding point. The force needed to change this hydrophobic surface was determined to contribute by $\sim 210 \mathrm{pN} \hat{=} 10 \%$ to the yielding force. To conclude, water compression is not expected to affect the observed forces, whereas a change of the hydrophobic surface contributed by $10 \%$ to the observed forces.

Additionally, the frictional forces acting on the tip-sphere were calculated and compared with results from the Stokes equation. It turned out, that the simulation results match the calculated values from the Stokes equation very well. For a probevelocity of $0.01 \mathrm{~nm} / \mathrm{ps}$, friction effects contribute to the observed forces by approx. $10 \%$ of the yielding forces.

Force-probe simulations were carried out at different probe-velocities. Unexpectedly, both elastic constants and yielding forces were found to depend logarithmically on probe-velocity. Similar logarithmic rate-dependencies of mechanical properties were also observed for other materials such as metallic glasses [147]. To account for this peculiar behavior, a new model adapted from relaxation dynamics perpendicular to the reaction coordinate was proposed and corroborated using Monte Carlo simulations.

For bacteriophage $\phi 29$, Ivanovska et al. did not observe a change of elastic constants for increasing probe-velocities in AFM experiments, only an increase of the yielding force was seen [43]. In contrast to our simulations, the indentation depth at which fracture occurred increased with probe-velocities of the AFM tip, correlating with a 
higher yielding force. This translates into a shift of the yielding point in the forcedistance plot towards larger indentations, which is consistent with an unchanged slope - and, thus, elastic constant - despite increasing yielding force.

AFM experiments on coated murike leukemia virus [29] and $\phi 29$ bacteriophage [43] yielded elastic constants in the range of $0.16-0.68 \mathrm{~N} / \mathrm{m}$, about one order of magnitude smaller than the elastic constants determined from our simulations. With respect to the maximum applied force measured by Ivanovska et al., our yielding forces are about $1.0 \mathrm{nN}$ larger than in the AFM measurements on $\phi 29$. We attribute this discrepancy to different probe-velocities. In AFM experiments, the approach-velocity of the tip is about five to seven orders of magnitude slower than in our simulations (Kol et al.: $0.095 \cdot 10^{-6} \mathrm{~m} / \mathrm{s}$ [29], Ivanovska et al.: approx. $1.0 \cdot 10^{-5} \mathrm{~m} / \mathrm{s}$ [43]). Extrapolating to the experimental velocities of $1.0 \cdot 10^{-5} \mathrm{~m} / \mathrm{s}$ yields elastic constant of $0.63 \mathrm{~N} / \mathrm{m}$ and yielding forces of $0.18 \mathrm{nN}$. The remaining smaller discrepancies of the yielding forces are attributed to the differing tip sizes; indeed, our simulations showed that the yielding forces increase markedly with tip size, in contrast to the elastic constants, which are independent of the tip-sphere size, as long as the tip is much smaller than the viral subunits.

Besides the linear response in the force-time dependence, we characterized the structural deformations in the rearrangement regime. It turned out that even this regime was dominated by elastic deformations of the complete proteins, subunits, and residues. Only close to the yielding point the cross-over to plastic deformation occurred. This high elasticity is remarkable and seems to be a property specific for viral shell proteins. Indeed, in force-probe simulations of the globular titin-kinase protein domain (PDB code: 1tit), in which the tip-sphere was pushed against and through the protein with a probe-velocity of $0.01 \mathrm{~nm} / \mathrm{ps}$ in a similar manner as the simulations described here (chapter 7), plastic deformations were seen already at the transition from linear to non-linear force increase in the force-time curve, much earlier than seen for the viral proteins.

On the atomic level, the linear regime is characterized by a deformation of an otherwise topologically unchanged conformation of neighboring atoms. In contrast, structural changes in the rearrangement regime are seen, with marked distance changes between nearest and next nearest neighbors. Thus, elastic deformation of the capsid is characterized by a collective motion of the complete protein or subunit as proposed earlier by Tama et al. [26], whereas plastic yielding involves rearrangements of adjacent single atoms or residues as well as of next nearest neighbors. No buckling transition of the virus was observed, probably due to the small tip size used here. Fracture only occurred locally at the position where the tip-sphere penetrated the viral shell.

Beyond the SBMV virus studied here, it would be interesting to disentangle which of the mechanical properties obtained depend on intramolecular properties of the involved viral shell proteins, on the local contact and geometry of the individual subunits, pentamers, or the overall icosahedral geometry of the complete capsid. Comparative studies of the viral shell of Human Rhinovirus 16 (see chapter 6) with the simulation methods established in this work will shine light on this issue. 


\section{The mechanical properties of Human Rhinovirus 16}

\subsection{Introduction}

Human Rhinoviruses (HRV) are one of the major causes of the common cold. 40$50 \%$ of all cold infections are caused by one of the over 100 serotypes of rhinovirus [148]. This huge diversity of rhinoviruses has made it impossible to produce effective vaccines.

Rhinoviruses belong to the picornaviruses which comprise one of the largest families of viral pathogens and are among the smallest RNA animal viruses with relative molecular mass of $\sim 8.5 \cdot 10^{6}$ and $\sim 30 \%$ by weight RNA. The protein shells have an external diameter of about $30 \mathrm{~nm}$ and form icosahedral capsids. Picornaviruses are divided into nine genera [149]. Four examples are enterovirus (e.g. polio, hepatitis A), cardiovirus (e.g. Mengo virus), aphthovirus (e.g. foot-and-mouth disease virus (FMDV)) and rhinovirus.

All picornavirions are built from 60 asymmetric subunits, thus called protomers with four proteins VP1, VP2, VP3 and VP4 [150] each. All virions contain a single, positive strand RNA which is translated into a single polyprotein, and then processed stepwise into its component proteins [151]. The gene order is essentially the same for HRV, FMDV, and poliovirus.

Rhinovirus serotypes are divided into three groups for the different receptors that bind to the viral shell [152-154]: the major rhinovirus receptor group which uses intercellular adhesion molecule-1 (ICAM-1, [155, 156]) as the receptor, e.g. HRV 14 [131, 157, 158] and HRV 16 [159-162], the minor rhinovirus receptor group, containing ten rhinoviruses which use a different receptor than ICAM-1, e.g. HRV 1A $[163,164]$, and HRV87 which uses a third receptor. Notice that although HRV 14 and HRV 16 use the same receptor, the activity of HRV 16 with respect to capsid-binding antiviral agents is more like that of HRV 1A [165].

Human Rhinovirus 16 (HRV 16) is a typical representative of the major receptor group which has been used in clinical tests [166] and is now in focus of our investigations. Its atomic structure was first resolved by x-ray crystallography in 1993 [162] and refined in 1997 [61] with a resolution of $2.15 \AA$. The capsid is composed of three external viral proteins VP1, VP2 and VP3, each with a molecular weight of approx. 30,000 Da. Protein VP4 is the fourth and smallest protein (molecular weight $7,000 \mathrm{Da}$ ) which is located at the internal surface of the capsid and in direct contact with the RNA (Fig. 6.3). 60 copies of these protomers, consisting of proteins VP1-VP4, form an icosahedral capsid with a pseudo $\mathrm{T}=3$ symmetry, also 


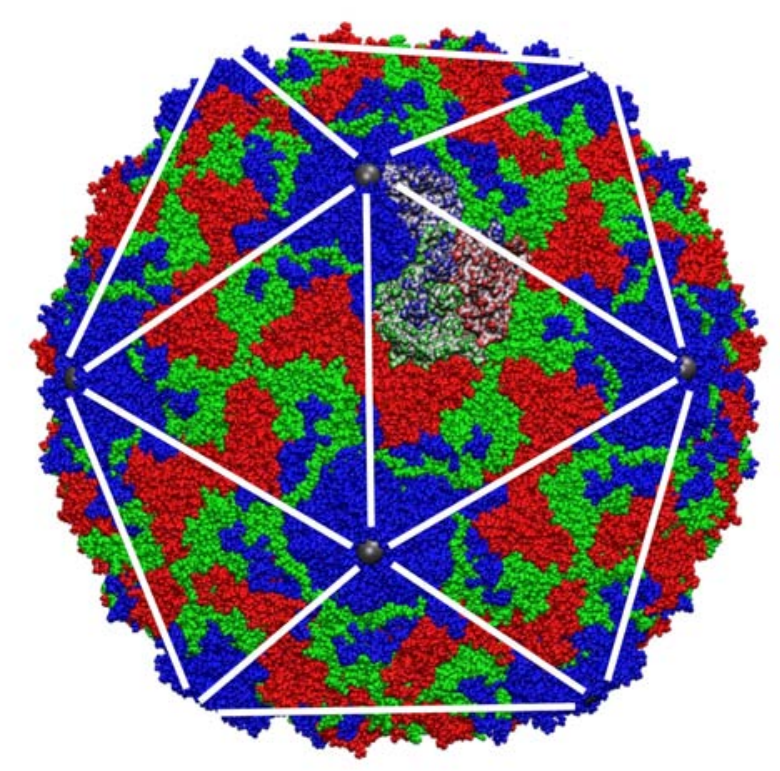

Figure 6.1: Human Rhinovirus 16, demonstrated shell geometry: $\mathbf{T}=\mathbf{1}$ symmetry. Two of the 12 pentamers, consisting of five facets (white lines)) each, around the zinc ions (gray spheres) at the 5-fold symmetry axis are emphasized with respect to a $\mathrm{T}=1$ symmetry. Here the interactions are identical between all 20 equilateral triangular facets. Protein VP1 is shown in blue, VP2 in red and VP3 in green. VP4 is not seen because it is located on the inner capsid surface. One of the 60 protomers is emphasized by a gray background surface.

labeled as $\mathrm{p}=3$, because the proteins are not chemically identical as for $\mathrm{T}=3$ capsids. Mathematically a picornavirus must be regarded as a heterotrimer on a $\mathrm{T}=1$ lattice (Fig. 6.1 and 6.2) [83]. Here the 3-fold symmetry axes are called pseudo 3 -fold axes. Compared to $\mathrm{T}=3$ viral shells, the similarity of the coat proteins is with VP1=A, VP2 $=\mathrm{C}$ and VP3=B (see subunits of Southern Bean Mosaic Virus, Fig. 4.2). Geometrically, protomers are not identical with subunits as depicted in Fig. 6.4. Inside the subunit, the four protomer proteins are arranged to form one of the 60 triangular facets. Furthermore, the shape of HRV 16 is more spherical than SBMV where the pentamers and hexamers are pronounced more clearly due to the symmetric and triangular subunits of SBMV.

Similar to SBMV and other RNA viruses, the three major coat proteins VP1-VP3 are folded into an eight stranded antiparallel $\beta$-barrel motiv (Fig. 6.3) [167, 168]. In 1985 when the structure of HRV 14 was already known, Rossmann et al. reported that the radial position and orientation of structurally equivalent $\mathrm{C}_{\alpha}$ atoms of $\mathrm{HRV}$ 14 and SBMV agree to better than $3 \AA$ relative to the icosahedral symmetry axes [131]. The 5-fold symmetry axes of all rhinoviruses are surrounded by a $\sim 12-20 \AA$ 


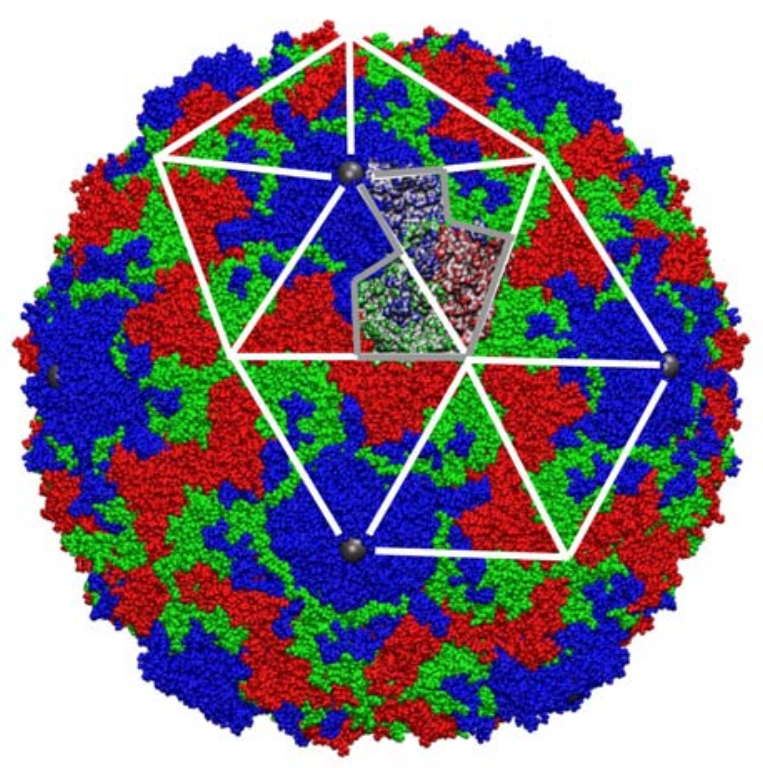

Figure 6.2: Human Rhinovirus 16, demonstrated shell geometry: $\mathbf{p}=\mathbf{3}$ symmetry. Nine of the 60 equilateral triangular facets are marked by white lines. One pentamer and one hexamer consisting of five and six subunits, respectively, are marked with respect to a pseudo $\mathrm{T}=3$ symmetry: $\mathrm{p}=3$ symmetry. The 2-, 3- and 5-fold symmetry axes are arranged identically to SBMV. The pentamers surround the 5 -fold symmetry axes where the zinc ions are located and the hexamers the pseudo 3 -fold axes. One protomer is emphasized by the gray lines.

deep canyon located in the VP1 proteins (Fig. 6.4) [169, 170]. The surface of the canyon is supposed to be the most conserved area of the viral coat protein which is inaccessible to antibodies due to steric hindrance.

Except icosahedral plant viruses, RNA animal viruses such as poliovirus and all serotypes of HRV have a hydrophobic pocket below the canyon in the VP1-protein which role in viral infectivity remains unknown [171]. The antiviral agents bind to the pocket to block uncoating of the viral particles and RNA release [172, 173, 27] and, in cases of some rhinoviruses like HRV 16, also inhibit receptor attachment [174]. X-ray crystallography studies showed that the pocket is empty for HRV 14 and, in case of HRV 16, occupied by a fatty acid-like molecule $\left(\mathrm{C}_{12} \mathrm{O}_{2} \mathrm{H}_{22}\right)$, the socalled pocket-factor DAO [61]. Oliveira et al. [162] proposed that binding of the receptor to HRV 16 can only occur when the pocket is temporarily empty which causes a destabilization of the capsid structure [131, 175]. They further claimed that binding of ICAM-1 traps the pocket in the empty state, destabilizing the virus by a collapse or "closing" of the open pocket to promote RNA release [61].

Each of the twelve 5-fold symmetry axes are capped with a $\mathrm{Zn}^{2+}$-ion, similar to the ion channel found in the SBMV shell [125]. The ion is assumed to play a key role in 


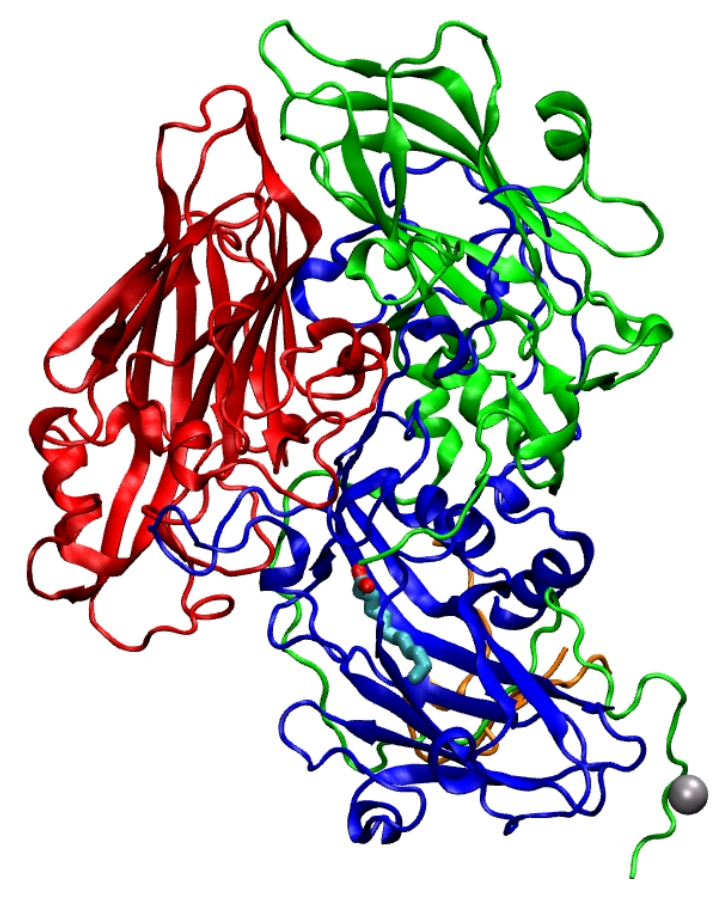

Figure 6.3: Human Rhinovirus 16 protomer. Protein VP1 is shown in blue, VP2 in red, VP3 in green and VP4 in orange. The zinc ion (grey sphere) is located at the 5-fold symmetry axis, the pocket factor, a fatty acid, is depicted by the cyan carbon chain with the two red oxygen atoms.

holding the neighboring VP1 proteins together at the pentamer center [61]. Besides the destabilization of the HRV 16 coat protein caused by a collapse of the pocket or the release of $\mathrm{Zn}^{2+}$ ions, it is known that the first stage in viral infection is the loss of the VP4 protein by incubation of the receptor $[176,177]$. The release of VP4 is accompanied by the externalization of the N-terminus of VP1. A possible pathway for VP4 exit would be the ion channel along the 5-fold symmetry axes. The function of the pentamer as port for RNA during disassembly of HRV 16 was discussed before for SBMV and is supposed to follow the same mechanism; an alternative way of uncoating for picornaviruses could be the separation of the building blocks [178].

In this work, we focus on the mechanical properties of the HRV 16 coat protein and three structural variants of HRV 16 containing a modified shell due to mutations in the capsid structure. In order to study the distribution of elastic properties of HRV 16 and its variation with structural changes of the capsid proteins, four simulation systems were considered for force-probe simulations: 


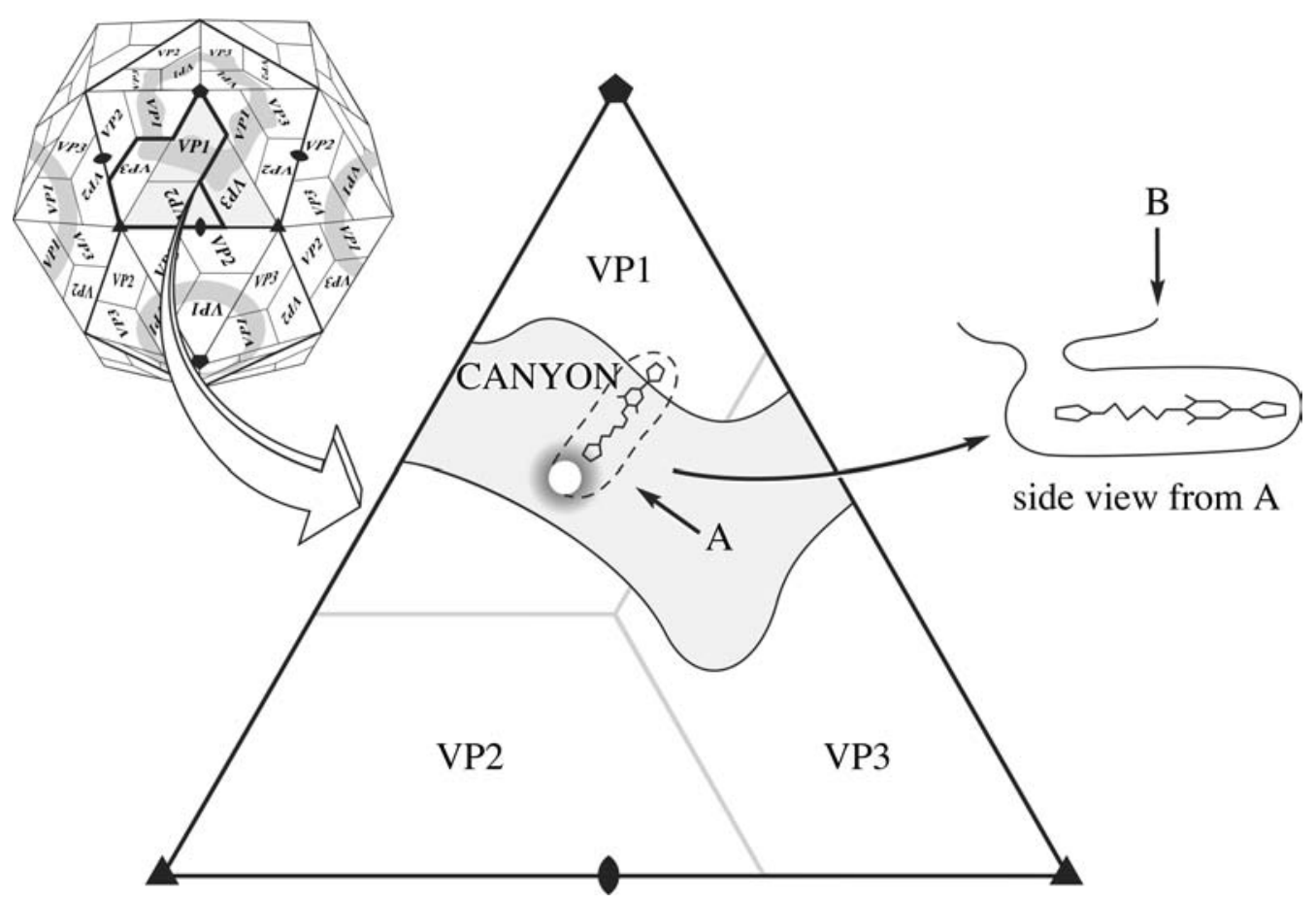

Figure 6.4: Scematic representation of a Human Rhinovirus with enlargement of one icosahedral asymmetric subunit showing the outline of the canyon and the entrance to the antiviral-binding pocket with the pocket-factor inside. The protomer which differs from the geometric definition of the subunit, is shown in heavy outline on the icohedron (top left). (Figure taken from [162].)

- Simulation system 1: Complete capsid with the pocket factor DAO

- Simulation system 2: Complete capsid without DAO

- Simulation system 3: Complete capsid without protein VP4

- Simulation system 4: Complete capsid without zinc ions

From the equilibration simulations of the different simulation systems, we determined the structural changes of the proteins caused by the mutations. Subsequently, the different capsids were indented with a tip-sphere on 19 evenly distributed grid points on the inner and outer shell of subunit 35. The obtained elasticity maps were compared for the four simulation systems, as well as with the results found from the force-probe simulations of SBMV. 


\subsection{Simulation details}

\subsubsection{Molecular dynamics simulations}

The x-ray structure of Human Rhinovirus (HRV) 16 at $2.15 \AA$ resolution was taken from the PDB (code: 1AYN [61]) and VIPER data bank. In order to study mutations of the HRV coat protein, for one simulation system the fatty acid DAO was removed of the structure, for a second system the protein VP4 was removed. As described for the simulation setup for Southern Bean Mosaic Virus, protons were added to each of the three HRV protein structures (chapter 6.2.3) with subsequent solvation in TIP4P water and the addition of sodium and chloride ions (chapter 3.1.4). The missing residues in the loop of protein VP4 were modeled into the simulation system with ModLOOP [179]. The simulation system of the complete structure of HRV 16 contained 762,312 protein atoms, including $12 \mathrm{Zn}^{2+}$ ions, $4842 \mathrm{Na}^{+} / \mathrm{Cl}^{-}$ions and approx. 860,000 water molecules, which together sum up to more than 4,200,000 atoms.

Each of the three systems was first energy minimized with 300 steepest decent steps. Subsequently, molecular dynamics simulation runs at $300 \mathrm{~K}$ equilibrated the systems as follows: All systems were coupled to a heat bath with $\tau_{T}=0.005 \mathrm{ps}$, with no pressure coupling applied. A 50 ps MD simulation with integration time steps of 0.5 fs and harmonically constrained heavy protein atoms with a force constant of $k=$ $1000 \mathrm{~kJ} \mathrm{~mol}^{-1}$ was performed. For the subsequent $50 \mathrm{ps}$ simulation, the integration time steps and the temperature coupling constant were increased to $\Delta t=1.0 \mathrm{fs}$ and $\tau_{T}=0.01 \mathrm{ps}$, respectively. Further $500 \mathrm{ps}$ were performed with $\Delta t=2.0 \mathrm{fs}$ and all bonds constrained using the LINCS algorithm [91]. The constraints on the heavy protein atoms were released, the integration time steps decreased to $\Delta t=$ $0.5 \mathrm{fs}$ without LINCS applied and all system equilibrated for $25 \mathrm{ps}$. Subsequently, the integration steps were increase to $\Delta t=1.0 \mathrm{fs}$ for $50 \mathrm{ps}$ and $\Delta t=2.0 \mathrm{fs}$ for $500 \mathrm{ps}$ with all bonds constrained with LINCS. Finally, the temperature coupling was weakened to $\tau_{T}=0.1 \mathrm{ps}$, and the pressure coupling was applied with $\tau_{p}=1.0 \mathrm{ps}$ to keep the pressure at 1 bar. All three simulation systems were equilibrated for several nanoseconds (see below) to ensure proper relaxation of the protein and water molecules.

After $15.5 \mathrm{~ns}$ of equilibration of the complete HRV 16 structure, the 12 zinc ions were removed from the simulation system. Counter ions were placed in the water around the protein to neutralize the simulation system which was subsequently equilibrated for $8 \mathrm{~ns}$ with the same MD parameters as described for the last equilibration steps for the other systems above.

During the equilibration phase, the root mean square deviation (rmsd) of the heavy protein atoms compared to the initial structures of the four systems was monitored, as well as the radii of gyration. 


\subsubsection{Force-probe molecular dynamics simulations}

In order to study the distribution of mechanical properties on the shell of HRV 16 and its three mutants, the force-probe simulation method established in the study on SBMV was employed. 19 grid points were chosen on the surface of subunit (SU 35) which consists of protein VP1, VP2 and VP4 of protomer 35 and VP3 of protomer 34 (Fig. 6.5, see also Fig. 6.4).

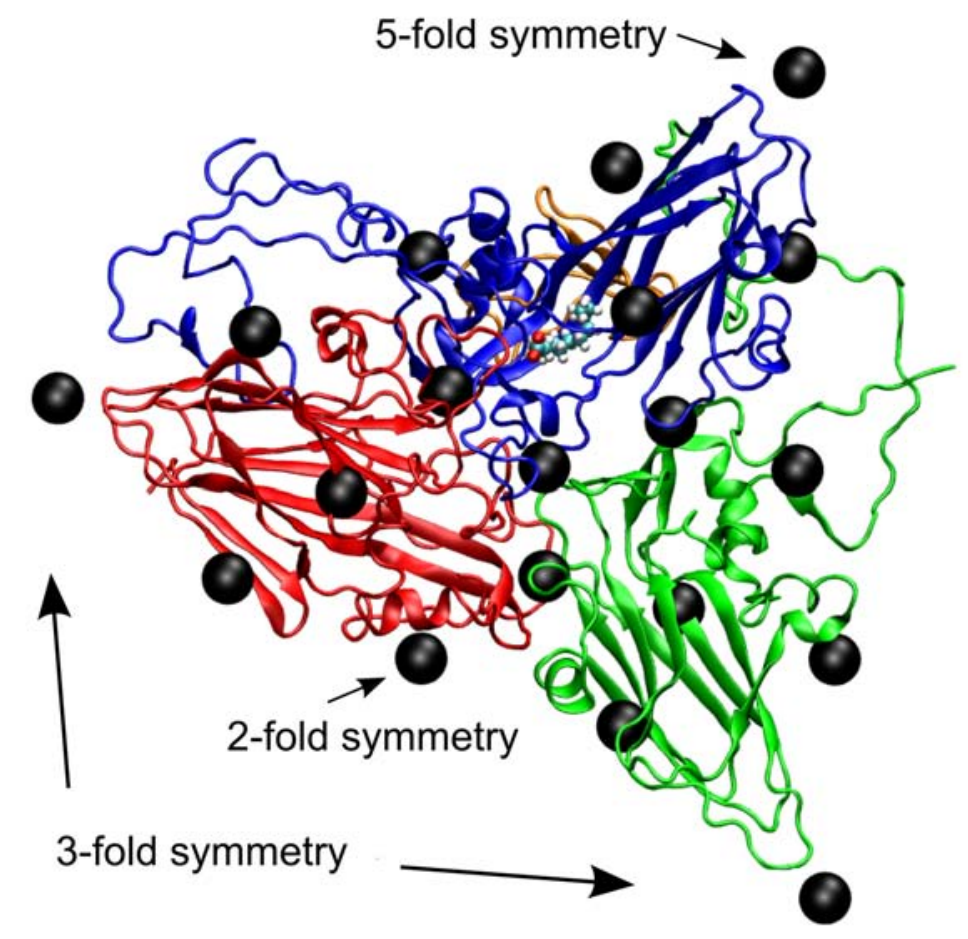

Figure 6.5: Subunit 35 of HRV 16. Blue: Protein VP1 of protomer 35 with pocket factor inside. Red: VP2 of protomer 35, Green: VP3 of protomer 34. Orange: VP4 of protomer 35. Black: grid points.

First, each grid point on the outer surface of each of the four simulation systems was indented twice with the tip-sphere with a probe-velocity of $0.01 \mathrm{~nm} / \mathrm{ps}$ as described in chapter 3.2. For the complete HRV 16 structure, the first series was performed from the structure extracted after $14.5 \mathrm{~ns}$ of equilibration, the second series after $15.5 \mathrm{~ns}$. The two force-probe simulation series of the mutants were performed from structures as follows. HRV 16 without pocket factor: $14.5 \mathrm{~ns}$ and $15.5 \mathrm{~ns}$. HRV 16 without VP4: $30 \mathrm{~ns}$ and $31 \mathrm{~ns}$. HRV without zinc ions: $7 \mathrm{~ns}$ and $8 \mathrm{~ns}$ after ion removal from the complete structure after $15.5 \mathrm{~ns}$.

Next, the four capsid structures were indented on the inner surface. Only five grid points were chosen for these simulations due to the enormous computing time needed for a complete indentation series on 19 grid points. Here, the tip-sphere was pushed 
along the 5-fold symmetry axis through the pentamer center, along the two quasi3 -fold symmetry axes through the hexamer centers, along the 2-fold symmetry axis, and through the subunit center where protein VP1, VP2 and VP3 meet. These force-probe simulations were also performed twice to rule out anecdotal events.

To study the relaxation behavior of the capsid, for the force-probe simulations in which the tip-sphere was pushed from outside along the 5- and 2-fold symmetry axes of the complete HRV 16 structure, the external force acting upon the tip-sphere was switched off at different times. Subsequently, the system was equilibrated for $500 \mathrm{ps}$ with free MD simulations.

For the simulations in which the tip-sphere was initially positioned outside the four capsid structures, a total of 152 indentation simulations was performed. When the tip-sphere was pushed against the inner capsid surfaces, 40 force-probe simulations were carried out. Molecular dynamics and force-probe simulations sum up to $220 \mathrm{~ns}$ of simulation time.

\subsubsection{Force field parameters for the fatty acid DAO}

No force field parameters for the fatty acid DAO in the HRV 16 structure were available for the OPLS-AA force field. Due to the similarity of the atom structure of DAO, $\mathrm{C}_{12} \mathrm{O}_{2} \mathrm{H}_{22}$, (Fig. 6.6) with the amino acid glutamine (GLU), the force field parameters for DAO were derived from the parameters for GLU, and are listed in table 6.1. Here the hydrogen atoms HA1, HA2 and HA3 are bonded to the carbon atom $\mathrm{CA}, \mathrm{HB} 1$ and $\mathrm{HB} 2$ to atom $\mathrm{CB}$, etc.

a)

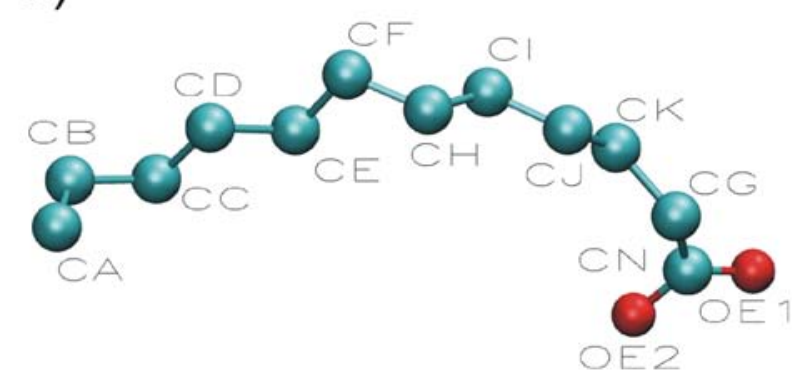

b)

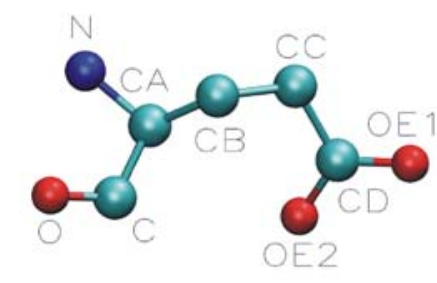

Figure 6.6: Structure of the fatty acid DAO (a) and glutamine (b). Carbon atoms are depicted in cyan, oxygen atoms in red, nitrogen in blue, hydrogen atoms are not shown. 


\begin{tabular}{|c|c|c|c|}
\hline ATOM TYPE & OPLS NAME & CHARGE & CHARGE GROUP \\
\hline $\mathrm{CA}$ & opls_135 & -0.180 & 1 \\
\hline HA1 & opls_140 & 0.060 & 1 \\
\hline HA2 & opls_140 & 0.060 & 1 \\
\hline HA3 & opls_140 & 0.060 & 1 \\
\hline $\mathrm{CB}$ & opls_136 & -0.120 & 2 \\
\hline HB1 & opls_140 & 0.060 & 2 \\
\hline HB2 & opls_140 & 0.060 & 2 \\
\hline $\mathrm{CC}$ & opls_136 & -0.120 & 3 \\
\hline $\mathrm{HC} 1$ & opls_140 & 0.060 & 3 \\
\hline $\mathrm{HC} 2$ & opls_140 & 0.060 & 3 \\
\hline CD & opls_136 & -0.120 & 4 \\
\hline HD1 & opls_140 & 0.060 & 4 \\
\hline HD2 & opls_140 & 0.060 & 4 \\
\hline $\mathrm{CE}$ & opls_136 & -0.120 & 5 \\
\hline HE1 & opls_140 & 0.060 & 5 \\
\hline HE2 & opls_140 & 0.060 & 5 \\
\hline $\mathrm{CF}$ & opls_136 & -0.120 & 6 \\
\hline HF1 & opls_140 & 0.060 & 6 \\
\hline HF2 & opls_140 & 0.060 & 6 \\
\hline $\mathrm{CH}$ & opls_136 & -0.120 & 7 \\
\hline HH1 & opls_140 & 0.060 & 7 \\
\hline HH2 & opls_140 & 0.060 & 7 \\
\hline $\mathrm{CI}$ & opls_136 & -0.120 & 8 \\
\hline HI1 & opls_140 & 0.060 & 8 \\
\hline HI2 & opls_140 & 0.060 & 8 \\
\hline CJ & opls_136 & -0.120 & 9 \\
\hline HJ1 & opls_140 & 0.060 & 9 \\
\hline HJ2 & opls_140 & 0.060 & 9 \\
\hline $\mathrm{CK}$ & opls_136 & -0.120 & 10 \\
\hline HK1 & opls_140 & 0.060 & 10 \\
\hline HK2 & opls_140 & 0.060 & 10 \\
\hline $\mathrm{CG}$ & opls_274 & -0.220 & 11 \\
\hline HG1 & opls_140 & 0.060 & 11 \\
\hline HG2 & opls_140 & 0.060 & 11 \\
\hline $\mathrm{CN}$ & opls_271 & 0.700 & 12 \\
\hline OE1 & opls_272 & -0.800 & 12 \\
\hline OE2 & opls_272 & -0.800 & 12 \\
\hline
\end{tabular}

Table 6.1: OPLS force field parameters for the fatty acid DAO in the HRV 16 structure. 


\subsection{Results}

\subsection{Results}

\subsubsection{Equilibration}

The four simulation systems of the complete HRV 16 structure and the mutants without the pocket factor DAO, zinc ions and VP4 protein were first equilibrated with free MD simulations before the force-probe simulations were performed. The complete equilibration phases after the steepest decent steps were $15.5 \mathrm{~ns}$ for the complete HRV structure and the system without the fatty acid DAO, $8 \mathrm{~ns}$ after $\mathrm{Zn}^{2+}$ removal from the equilibrated HRV 16 structure, and $31 \mathrm{~ns}$ for the viral shell structure without VP4. The corresponding rmsd curves of the heavy protein atoms with respect to the initial structures are shown in Fig. 6.7.

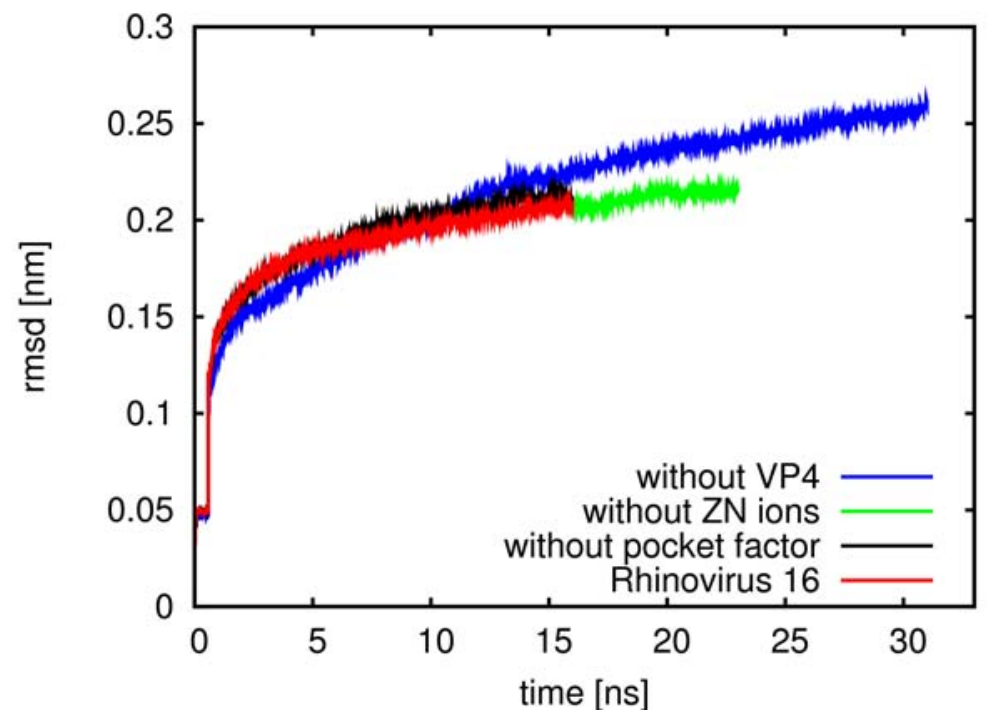

Figure 6.7: Rmsd curves for the HRV 16 and the three mutants during equilibration. The rmsd was fitted to the initial capsid structures after steepest decent steps.

While the position restraints were applied to the heavy protein atoms, the rmsd slightly increased to $0.5 \AA$. Subsequently, when the atoms were released, the rmsd of the HRV 16 structure and the structure without DAO increased to $1.85 \AA$ at $5 \mathrm{~ns}$ and $1.95 \AA$ (HRV 16) and $2.0 \AA$ (without DAO) at 10 ns. Both rmsd curves subsequently remained almost stable at $2.05 \AA$ (HRV 16) and $2.12 \AA$ (without DAO) after $14 \mathrm{~ns}$ until $15.5 \mathrm{~ns}$, the end of the equilibration phase. After the removal of the zinc ions from the complete capsid structure, the rmsd increased from initially $2.05 \AA$ to $2.18 \AA$ after $19 \mathrm{~ns}$ in total ( $\hat{=} 3.5 \mathrm{~ns}$ without $\mathrm{Zn}^{2+}$ ) at which the rmsd leveled off and remained constant until $23.5 \mathrm{~ns}$ of equilibration ( $\hat{=} 8 \mathrm{~ns}$ without $\mathrm{Zn}^{2+}$ ).

After 5ns of equilibration, an rmsd for the capsid without VP4 of $1.70 \AA$ was obtained. At $15 \mathrm{~ns}$, the rmsd increased to $2.23 \AA$ and to $2.48 \AA$ at $25 \mathrm{~ns}$. At the end of the equilibration phase at $31 \mathrm{~ns}$, an rmsd of $2.60 \AA$ was determined. Although 
the viral shell structure was not fully converged, the force-probe simulations were performed from this structure. We found from force-probe simulations of Southern Bean Mosaic Virus that even not fully converged structures exhibit similar mechanical properties compared to converged structures, and we therefore consider our four simulation systems sufficiently equilibrated to study their elastic constants and yielding forces.

Fig. 6.8 shows protein VP1 of protomer 35 after the $15.5 \mathrm{~ns}$ equilibration phase for the complete HRV 16 structure and the capsid protein without the pocket factor. Both structures exhibited an open pocket, and the removal of the fatty acid hardly changed the atomic structure of VP1. An rmsd of $1.1 \AA$ between the VP1 proteins with and without the pocket factor was determined (Fig. 6.8).

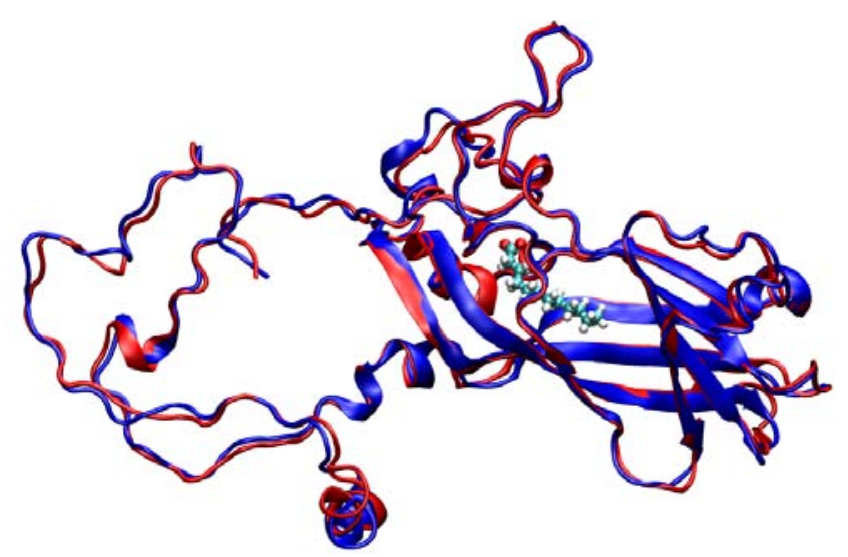

Figure 6.8: VP1 of protomer 35 after 15.5 ns of equilibration. Blue: Rhinovirus HRV 16 structure, red: Rhinovirus without pocket factor DAO. The pocket factor is shown in cyan with red oxygen atoms. Rmsd between the two structures: $1.1 \AA$.

After equilibration of the four simulation systems, the following rmsd values between the structure were determined:

- rmsd between HRV 16 and capsid without ZN: $1.3 \AA$.

- rmsd between HRV 16 and capsid without DAO: $2.1 \AA$.

- rmsd between HRV 16 and capsid without VP4: $2.6 \AA$.

Additionally, the radius of gyration of the capsids was monitored. A typical curve is given in Fig. 6.9 for the complete HRV 16 structure during equilibration. The radius of gyration slightly increased by approx. $0.12 \AA$ with respect to the $\mathrm{x}$-ray structure, which was also seen for the three mutants of the capsid. 


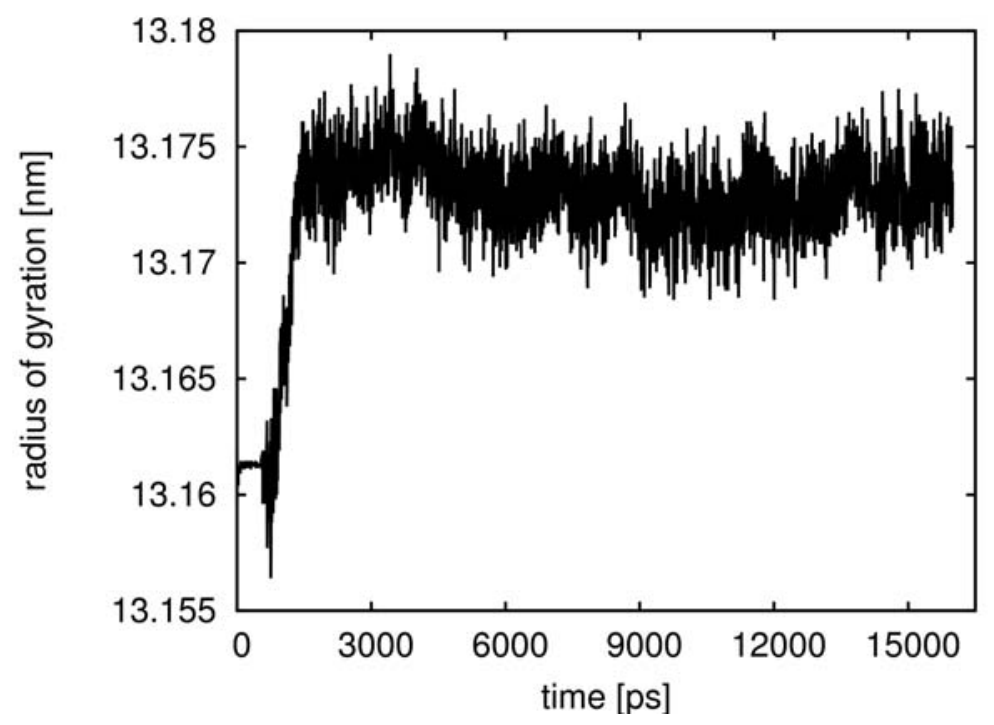

Figure 6.9: Radius of gyration of the Human Rhinovirus 16 coat protein during the equilibration phase. 


\subsubsection{Deformation of the capsids}

In order to study the distribution of elastic constants and yielding forces on the viral shells of Human Rhinovirus 16 and three mutants with a modified capsid structure, a tip-sphere was pushed against 19 evenly distributed grid points on subunit 35 . From each simulation, a force-distance and force-time curve was obtained for the force acting on the tip-sphere. Such a representative plot is given in Fig. 6.10.

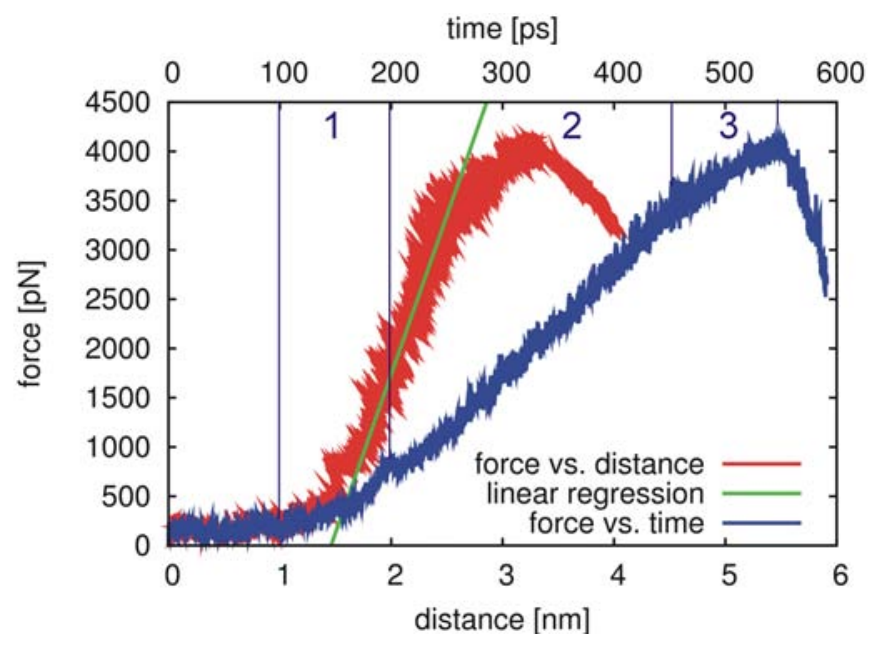

Figure 6.10: Force-distance (red) and force-time (blue) curve obtained from a simulation in which the tip-sphere was pushed against and through the center of protein VP2 of the shell of HRV 16. The pre-linear (1), linear (2) and rearrangement regime (3) of the force-time curve are marked. The green line depicts the slope of the linear regime, thus the elastic constant at the respective indentation position.

When the tip-sphere approached the capsid after $100 \mathrm{ps}$, the force started to increase (pre-linear regime (1) in Fig. 6.10) and bended over to a linear force-increase from 200-450 ps (linear regime (2)). The adjacent rearrangement regime yielded at a maximum force at $550 \mathrm{ps}$ before the force dropped, and the tip-sphere left the shell towards the direction of the viral center. The elastic constant was determined from the slope of the linear regime, the yielding force was obtained from the maximum force. A detailed description of the force-distance and force-time curves is given in chapter 6.3.5.

\subsubsection{Elastic constants}

From the four force-probe simulation series, each containing 38 simulations in which the tip-sphere was pushed against the 19 grid point positions on the outer surface of HRV 16 and its three mutants, a heterogeneous distribution of elastic constants was obtained (Fig. 6.11). The values varied between $1.75 \mathrm{~N} / \mathrm{m}$ and $4.72 \mathrm{~N} / \mathrm{m}$. 


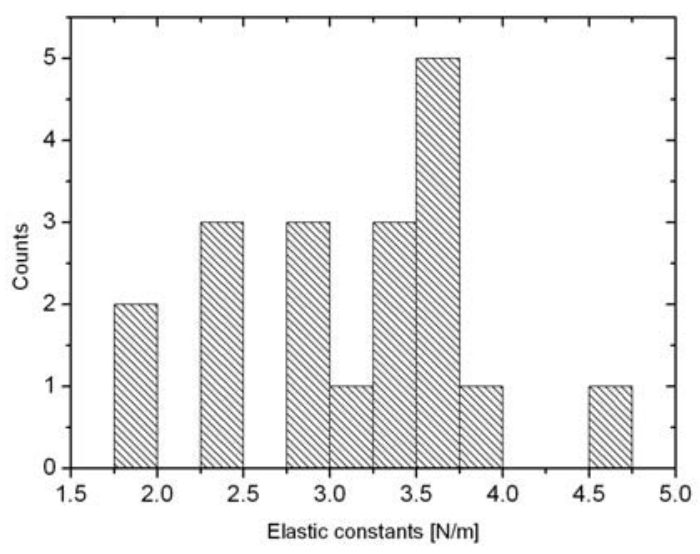

Figure 6.11: Histogram of elastic constants obtained from the 38 force-probe simulations in which the tip-sphere was directed towards the 19 grid points on the outer surface of the complete HRV 16 shell.

\section{Complete HRV 16 capsid}

The obtained elastic constants from the force-probe simulations of the complete HRV 16 structure are shown in Fig. 6.12. The smallest elastic constant was found at the subunit center $(1.75 \mathrm{~N} / \mathrm{m})$, and the largest elastic constant at the interface of protein VP1 and VP3 inside the subunit next to its center $(4.72 \mathrm{~N} / \mathrm{m})$. Along the 5 -fold symmetry axis at the pentamer center, the elastic constant $(2.89 \mathrm{~N} / \mathrm{m})$ was found to be smaller than the values along the 3 -fold symmetry axes $(3.30 \mathrm{~N} / \mathrm{m}$ and $3.47 \mathrm{~N} / \mathrm{m}$, respectively) and the 2 -fold symmetry axis $(3.51 \mathrm{~N} / \mathrm{m})$. Inside the subunit, the elastic constants obtained at the protein centers varied markedly (VP1: $3.38 \mathrm{~N} / \mathrm{m}, \mathrm{VP} 2: 3.60 \mathrm{~N} / \mathrm{m}, \mathrm{VP} 3: 2.81 \mathrm{~N} / \mathrm{m}$ ), whereas the distribution was even more heterogeneous at the protein interfaces $(1.79 \mathrm{~N} / \mathrm{m}$ at the interface of VP2 and VP3, $4.47 \mathrm{~N} / \mathrm{m}$ at the interface of VP1 and VP3, $2.25 \mathrm{~N} / \mathrm{m}$ at the interface of VP1 and VP2). Between subunit 35 and its adjacent subunits 20, 34 and 33 (Fig. 6.12), the smallest elastic constants were seen in the middle of the interfaces of subunit 35-20 and $35-34(2.34 \mathrm{~N} / \mathrm{m}$ and $2.45 \mathrm{~N} / \mathrm{m}$, respectively). The values at the interfaces of VP1 proteins that surround the pentamer center were determined to be $2.86 \mathrm{~N} / \mathrm{m}$ and $3.01 \mathrm{~N} / \mathrm{m}$, respectively. The remaining elastic constants at the subunit interfaces varied between $3.51 \mathrm{~N} / \mathrm{m}$ (2-fold symmetry axis) and $3.75 \mathrm{~N} / \mathrm{m}$ (interface protein $\mathrm{VP} 2{ }_{35}$ and $\mathrm{VP} 3_{35}$ ).

It should be mentioned, that at the pentamer center, four force peaks were seen in the force-distance plot (Fig. 6.26a) with subsequent force drop. Although the height of these yielding forces differed, the first three respective linear regimes before yielding occurred, exhibited very similar slopes and thus elastic constants. Therefore only one value for the elastic constant is given for the respective grid point at the pentamer center although two yielding forces are depicted in Fig. 6.17 (see also chapter 6.3.5). 
a)

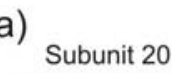

-fold sym. axis

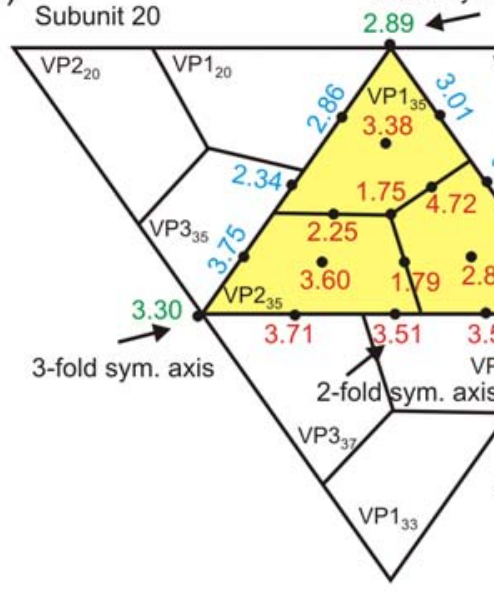

Subunit 33

Subunit 34 b)

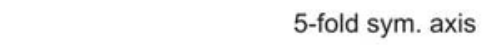

Figure 6.12: Elastic constants of rhinovirus HRV 16, complete shell, probed from outside. a) Sketch of SU 35 (yellow) and the adjacent subunits 20, 33 and 34 (white). The black dots mark the grid points at which the elastic properties were determined by force-probe simulations in which the tip-sphere was pushed against the capsid. The obtained elastic constants are shown in red, green, blue; the different colors of the numbers denote different push-vector directions perpendicular to the viral surface. b) Color-coded distribution of elastic constants on subunit 35, obtained from values shown in a) (soft: blue, stiff: red).

\section{Rhinovirus without fatty acid as pocket factor}

The distribution of elastic constants on the 19 different grid point positions became more homogeneous after the removal of the fatty acid from the pocket in protein VP1, and the values varied between $2.31 \mathrm{~N} / \mathrm{m}$ at the subunit center and $3.81 \mathrm{~N} / \mathrm{m}$ at the hexamer center (Fig. 6.13). Inside subunit 35, the elastic constants obtained at the protein centers were hardly affected by the fatty acid removal, whereas the values at the protein interfaces changed markedly, e.g. the elastic constant between VP1 and VP3 declined by $1.41 \mathrm{~N} / \mathrm{m}$ to $3.31 \mathrm{~N} / \mathrm{m}$. The subunit center became stiffer, and the elastic constant rose to $2.31 \mathrm{~N} / \mathrm{m}$. The elastic constants obtained along the different symmetry axes became larger, but nevertheless the pentamer center $(3.40 \mathrm{~N} / \mathrm{m})$ still exhibited the softest position compared to the hexamer centers $(3.81 \mathrm{~N} / \mathrm{m}$ and $3.74 \mathrm{~N} / \mathrm{m}$, respectively) and 2-fold symmetry axis $(3.74 \mathrm{~N} / \mathrm{m})$. Only slight deviations compared to the complete HRV 16 structure were found at the interfaces of this mutated subunit 35 structure to the adjacent subunits 20 and 34. The elastic constants between protein VP2 and VP3 at the interface of subunit 35 and 33 decreased by approx. $0.7 \mathrm{~N} / \mathrm{m}$ to $3.01 \mathrm{~N} / \mathrm{m}$ and $2.89 \mathrm{~N} / \mathrm{m}$, respectively. 
a)

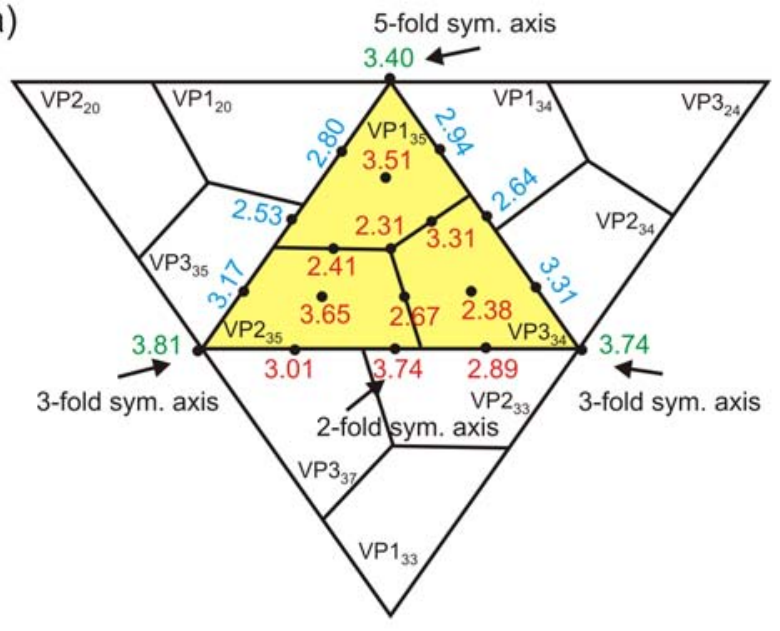

b)

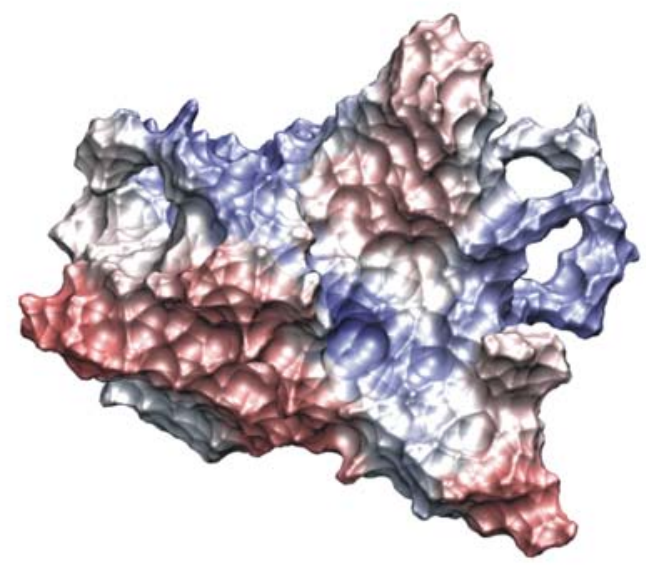

Figure 6.13: Elastic constants of rhinovirus without pocket factor (in N/m) and color-coded subunit 35 shown as in Fig. 6.12.

\section{Rhinovirus without zinc ions}

The elastic constants of subunit 35 did not change markedly after the removal of the zinc ions from the HRV 16 structure (Fig. 6.14). Only the value at the interface of protein $\mathrm{VP}_{35}$ and $\mathrm{VP} 3_{34}$ decreased to $3.36 \mathrm{~N} / \mathrm{m}$. The remaining elastic constants inside subunit 35 were unchanged. An increase of elastic constants was obtained along the 5 -fold symmetry axis $(3.29 \mathrm{~N} / \mathrm{m})$ and along the 2 -fold symmetry axis $(4.63 \mathrm{~N} / \mathrm{m})$ which became the stiffest position on the capsid surface. The values determined at the hexamer centers were slightly reduced compared to the complete HRV 16 structure $(3.01 \mathrm{~N} / \mathrm{m}$ and $3.09 \mathrm{~N} / \mathrm{m}$, respectively).

\section{Rhinovirus without protein VP4}

The removal of protein VP4 from the HRV 16 structure did not cause a change in elastic constants along the symmetry axis (Fig. 6.15). The obtained value at the subunit center increased to $2.34 \mathrm{~N} / \mathrm{m}$ and decreased between protein $\mathrm{VP} 1_{35}$ and $\mathrm{VP} 3_{34}(3.27 \mathrm{~N} / \mathrm{m})$, as already found for the elastic constants at these two grid points on the other mutated capsid structures. In contrast to the elastic constants at the interface of subunit 35 and 33 of HRV 16, a stiffness decrease was obtained here $(3.17 \mathrm{~N} / \mathrm{m}$ and $3.20 \mathrm{~N} / \mathrm{m}$, respectively). The remaining elastic constants inside subunit 35 and at the subunit interfaces hardly changed compared to the values obtained for the complete HRV 16 capsid. 
a)

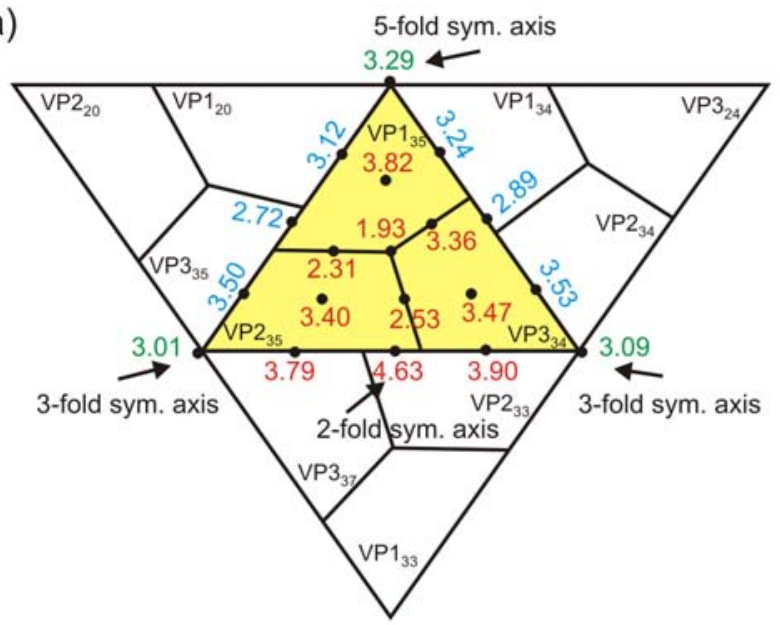

b)

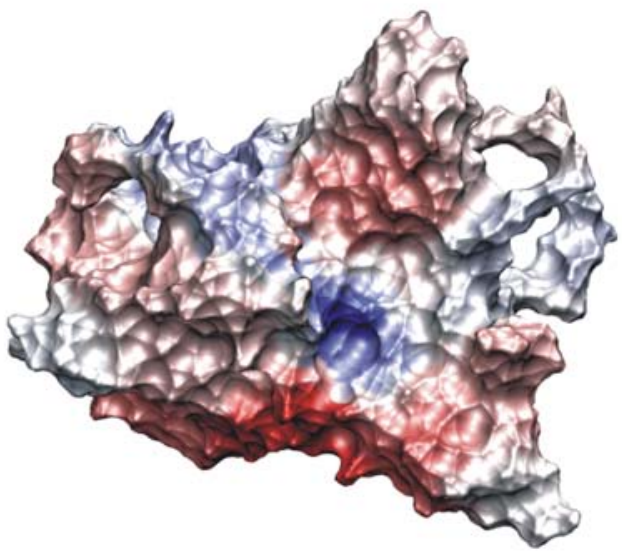

Figure 6.14: Elastic constants of rhinovirus without zinc ions (in $\mathrm{N} / \mathrm{m}$ ) and color-coded subunit 35 shown as in Fig. 6.12.

a)

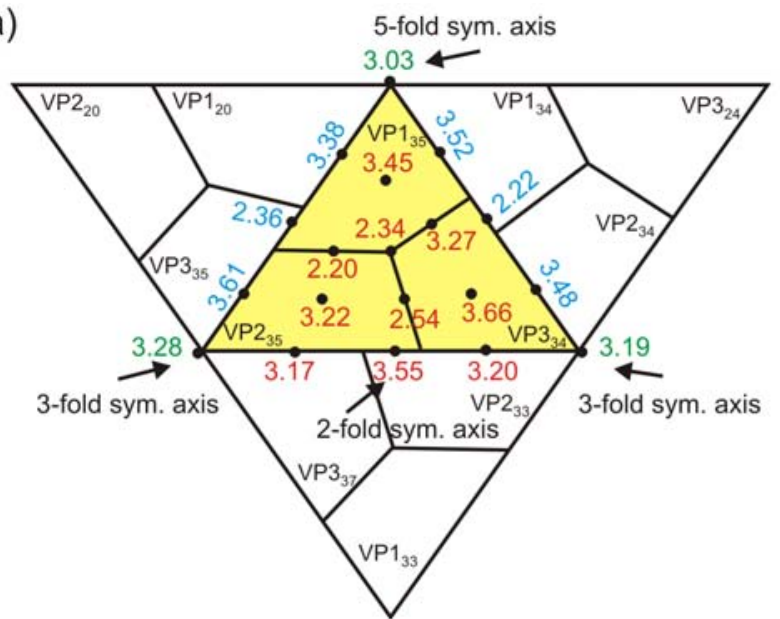

b)

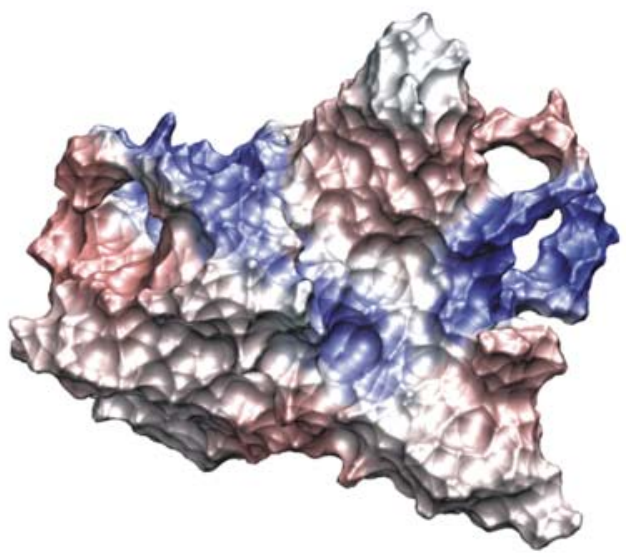

Figure 6.15: Elastic constants of rhinovirus without VP4 (in N/m) and colorcoded subunit 35 shown as in Fig. 6.12. 


\subsection{Results}

\section{Elastic constants on the inner capsid surface}

We performed force-probe simulations in which the tip-sphere was initially located inside the capsid and subsequently pushed against the inner surface of the viral shell. The complete shell of HRV 16 and the three mutants were probed on the grid points located along the 2-, 3- and 5-fold symmetry axes and the subunit center on the inner capsid surfaces. A total of $40 \mathrm{FP}$ simulations were carried out, from which the following elastic constants were obtained (Tab. 6.2).

Table 6.2: Elastic constants (in $\mathrm{N} / \mathrm{m}$ ) obtained on the inner surface of Human Rhinovirus 16 and its three mutants from grip points at the symmetry axes and the subunit center. The force-distance curves obtained from indentations of the pentamer center exhibited several linear regimes and, thus, more than one elastic constant was determined. The first value depicts the elastic constant of the first linear regime, the numbers in brackets are the largest values obtained from the largest slope of an adjacent linear regime in the respective force-distance plot.

\begin{tabular}{|c||c|c|c|c|}
\hline & RHINOVIRUS & WITHOUT ACID & WITHOUT ZN & WITHOUT VP4 \\
\hline \hline 5-fold axis & $3.40(4.20)$ & $2.30(3.12)$ & $1.72(5.23)$ & 3.67 \\
3-fold axis & 1.70 & 2.13 & 1.58 & 1.82 \\
2-fold axis & 3.71 & 3.49 & 4.04 & 3.24 \\
subunit center & 2.97 & 3.08 & 3.64 & 3.07 \\
\hline
\end{tabular}

The grid points at the 3-fold symmetry axes exhibited the smallest stiffness found from all four simulation systems; the elastic constants varied between $1.58 \mathrm{~N} / \mathrm{m} \mathrm{(mu-}$ tant without zinc) and $2.13 \mathrm{~N} / \mathrm{m}$ (mutant without fatty acid). The force-distance curve obtained from the simulations in which the tip-sphere was pushed along the 5 -fold symmetry axes, showed four peaks (Fig. 6.26a) with the exception of the mutant without protein VP4. Thus four elastic constants were seen for these grid points which were determined from the preceding slopes of the linear regimes before yielding occurred. The first value in Tab. 6.2 depicts the elastic constants determined from the first linear regime before the first yielding point was reached (Fig. $6.26 \mathrm{a}$ ), the second value in brackets depicts the largest elastic constant which was obtained from one of the three subsequent linear regimes that exhibited the largest slope of all linear regimes. It can be seen that the highest capsid stiffness was obtained at the pentamer centers $\left(2^{\text {nd }}\right.$ linear regime). Although two yielding forces for the indentation of the hexamer centers are shown in Tab. 6.3, only one elastic constant is given for these respective grid points in Tab. 6.2. The elastic constants obtained for the different linear regimes in the force-distance curves resulted in the same elastic constants within the estimated error.

The elastic constants at the subunit centers increased to $\sim 3 \mathrm{~N} / \mathrm{m}$ compared to the values at the outer capsid surface $(1.75 \mathrm{~N} / \mathrm{m})$, whereas the mutant without zinc exhibited an even larger elastic constant of $3.64 \mathrm{~N} / \mathrm{m}$. In summary, this mutant showed the most heterogeneous distribution of elastic constants on the inner capsid surface. 
Here, the values varied between $1.58 \mathrm{~N} / \mathrm{m}$ and $5.23 \mathrm{~N} / \mathrm{m}$, whereas after removal of the fatty acid from the HRV 16, a more homogeneous distribution of elastic constants with values between $2.13 \mathrm{~N} / \mathrm{m}$ and $3.12 \mathrm{~N} / \mathrm{m}$ was found.

The estimated standard deviation, determined from all force-probe simulations, was in the range of $0.14-0.36 \mathrm{~N} / \mathrm{m}$.

\subsubsection{Yielding forces}

In addition to the elastic constants, the yielding forces were determined from the same force-probe simulations on the complete HRV 16 structure and its three mutants as discussed before. A heterogeneous distribution of yielding forces (Fig. 6.16) was found for the 19 grid point positions on the outer capsid surface of subunit 35 with values ranging from $1.87 \mathrm{nN}$ to $5.09 \mathrm{nN}$.

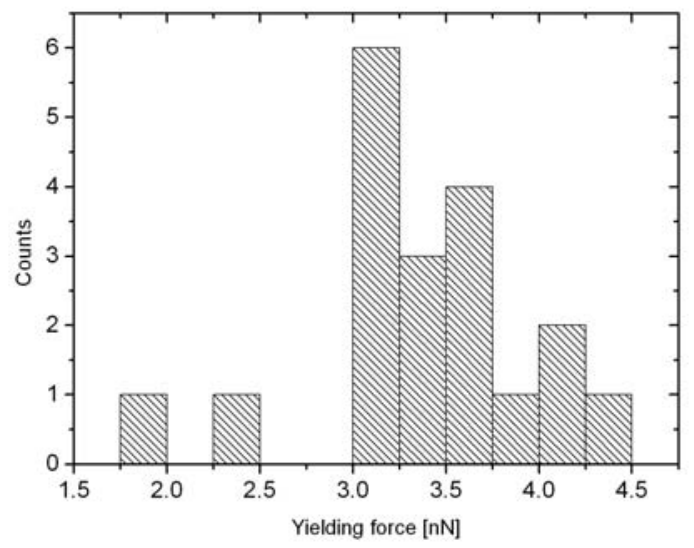

Figure 6.16: Histogram of yielding forces obtained for the complete HRV 16 structure as in Fig. 6.11.

\section{Complete HRV 16 capsid}

Fig. 6.17 shows the obtained yielding forces from the 38 force-probe simulations in which the tip-sphere was pushed against and through the 19 grip points on the outer capsid surface of HRV 16. The largest yielding force was seen along the 2-fold symmetry axis $(4.44 \mathrm{nN})$, the smallest along the 5 -fold symmetry axis, whereas here four yielding forces were obtained from the force-distance plot (Fig. 6.26a). The first force peak was seen at $1.87 \mathrm{nN}$, the largest value at the third peak at $2.82 \mathrm{nN}$. The forces obtained from peak two and three were always similar (see 6.3.5). The yielding forces at the hexamer centers were $3.07 \mathrm{nN}$ and $3.08 \mathrm{nN}$, respectively. The values at the protein centers inside subunit 35 were similar (VP1: $4.13 \mathrm{nN}, \mathrm{VP} 2$ : $4.12 \mathrm{nN}$ and VP3: $3.86 \mathrm{nN}$ ), the yielding forces between the proteins varied between 


\subsection{Results}

$3.04 \mathrm{nN}$ (VP2 and VP3) and $3.70 \mathrm{nN}$ (VP1 and VP2). The distribution of yielding forces at the grid points of the subunit interfaces was seen to be homogeneous, and the values were in the range of $3.11 \mathrm{nN}$ and $3.58 \mathrm{nN}$ (despite the value at the 2 -fold symmetry axis).

a)

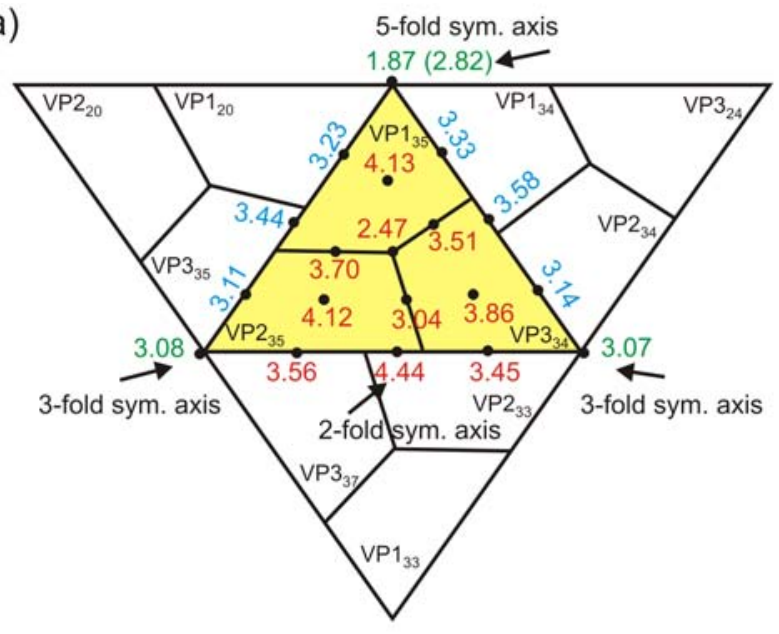

b)

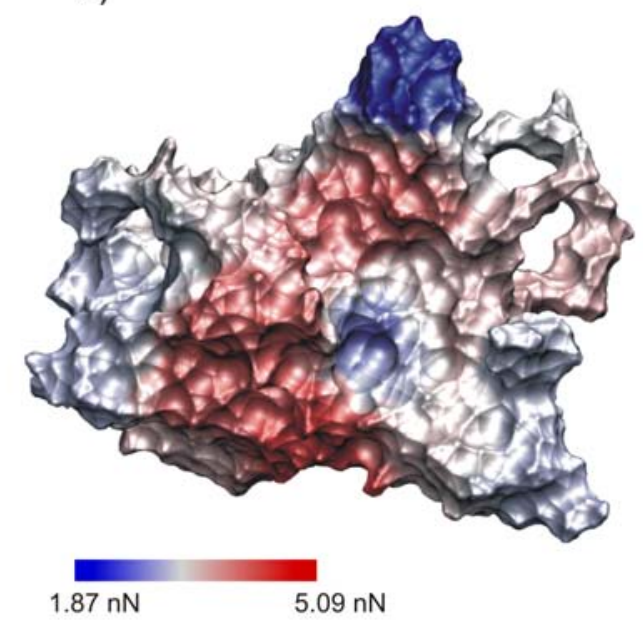

Figure 6.17: Yielding force of rhinovirus HRV 16, complete shell, probed from outside (in $\mathrm{nN}$ ) shown as in Fig. 6.12 (blue: low stability, red: high stability).

\section{Rhinovirus without fatty acid as pocket factor}

The removal of the fatty acid from the pocket in protein VP1 mainly changed the obtained yielding forces at the 2-fold symmetry axis (increase by $0.65 \mathrm{nN}$ to $5.09 \mathrm{nN}$ ) and at the subunit center (increase by $0.99 \mathrm{nN}$ to $3.46 \mathrm{nN}$, Fig. 6.18). The yielding forces seen at the 17 remaining grid points were hardly affected. The deviations from the values obtained for the complete HRV 16 structure were in the range of $0.01 \mathrm{nN}$ to $0.57 \mathrm{nN}$.

\section{Rhinovirus without zinc ions}

Changes of yielding forces after removal of the zinc ions from the HRV 16 structure were in the range of $0.06 \mathrm{nN}$ to $0.44 \mathrm{nN}$ with the exception of the subunit center (Fig. 6.19). Here, the yielding force rose to $3.23 \mathrm{nN}$. The grid point at the 2 -fold symmetry axis was slightly reinforced $(4.79 \mathrm{nN})$, whereas the yielding forces at the 3 -fold symmetry axes declined to $2.63 \mathrm{nN}$ and $2.79 \mathrm{nN}$, respectively. The value at the 5 -fold symmetry axis, where the zinc ion was originally located, remained unchanged. 
a)

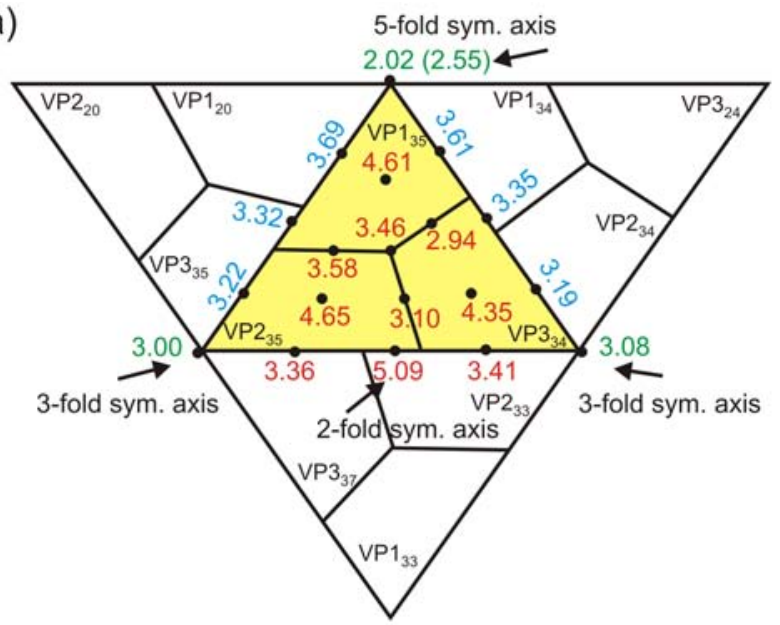

b)

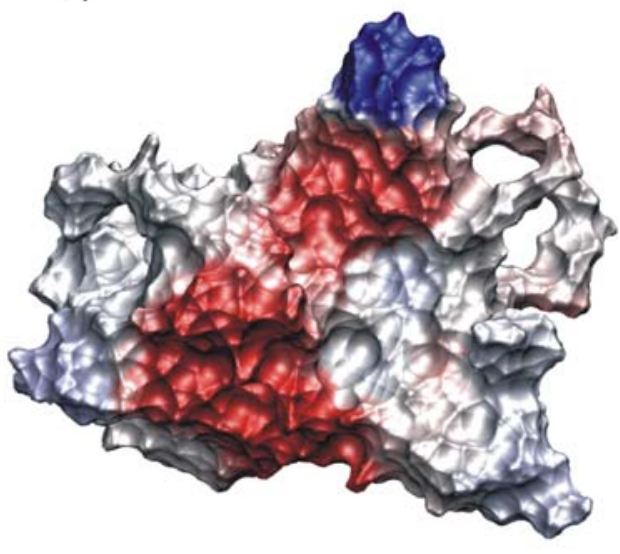

Figure 6.18: Yielding force of rhinovirus without acid (in $\mathrm{nN}$ ) shown as in Fig. 6.12 (blue: low stability, red: high stability).

a)

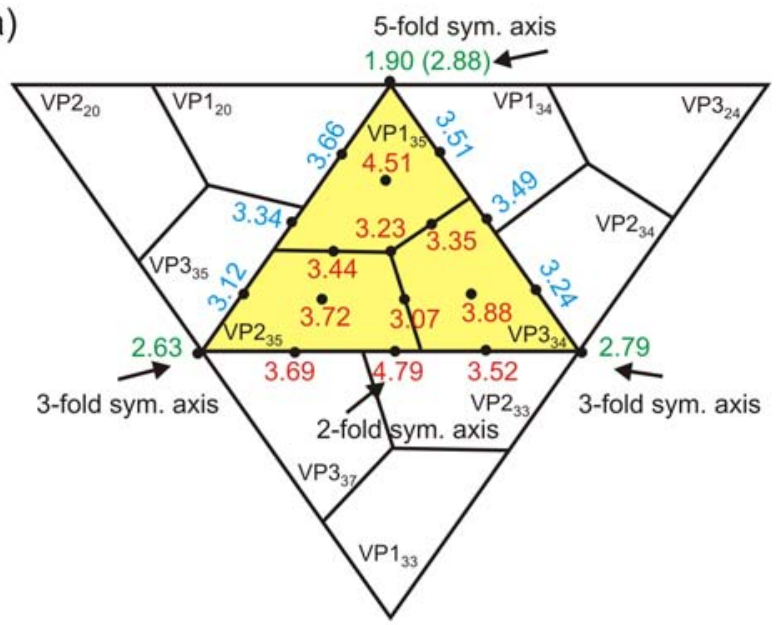

b)

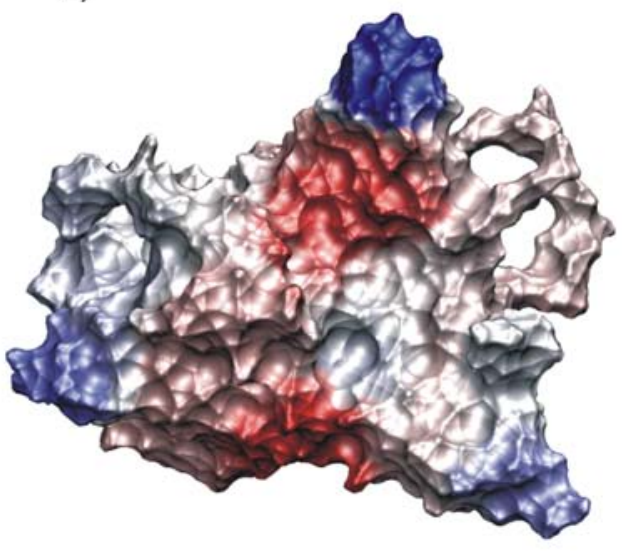

Figure 6.19: Yielding force of rhinovirus without ZN (in nN) shown as in Fig. 6.12 (blue: low stability, red: high stability). 


\subsection{Results}

\section{Rhinovirus without protein VP4}

Removal of the coat protein VP4 affected the yielding forces seen on the outer surface of the viral shell weakly (Fig. 6.20). The subunit center became stiffer, and a yielding force of $3.36 \mathrm{nN}$ was determined. The center of protein VP1 became less stable (force decreased to $3.65 \mathrm{nN}$ ). The remaining yielding forces obtained on the other grid points deviated by less than $0.43 \mathrm{nN}$ compared to the values seen for the complete HRV 16 structure.

a)

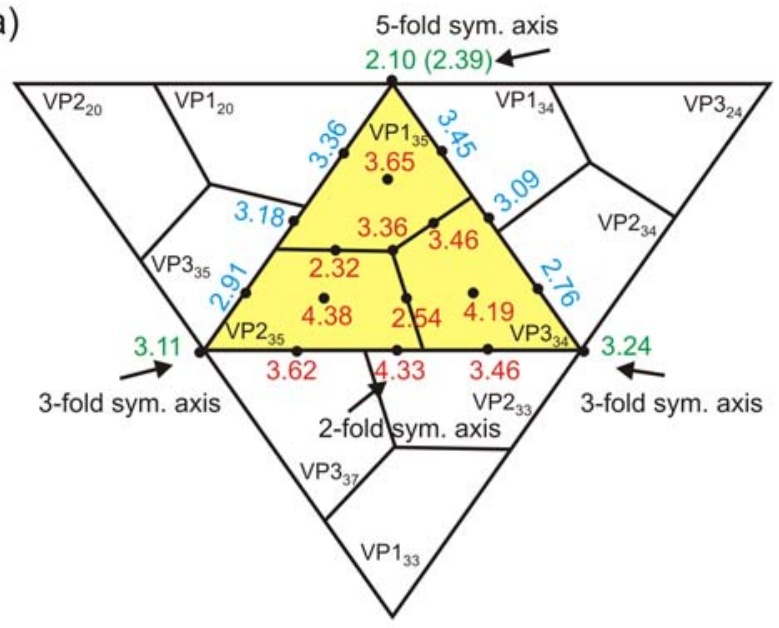

b)

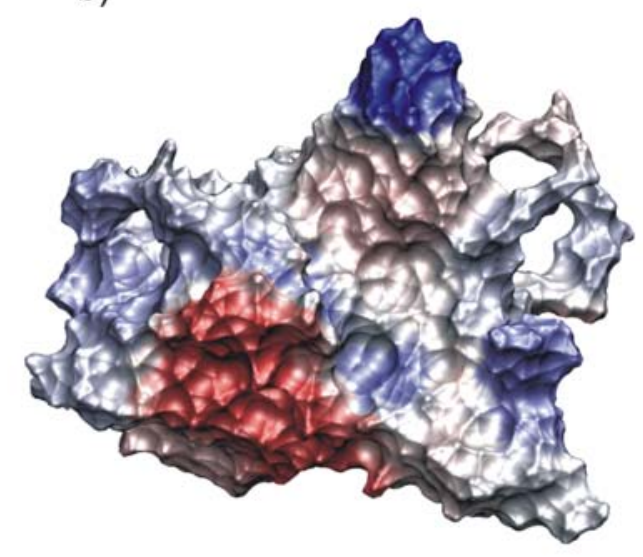

Figure 6.20: Yielding force of rhinovirus without VP4 (in nN) shown as in Fig. 6.12 (blue: low stability, red: high stability).

\section{Yielding forces on the inner capsid surface}

The yielding forces obtained at the grid points along the symmetry axes and the subunit center on the inner capsid surfaces of HRV 16 and its three mutants are shown in Tab. 6.3.

Table 6.3: Yielding forces (in $\mathrm{nN}$ ) obtained on the inner surface of Human Rhinovirus 16 and its three mutants from grip points at the symmetry axes and the subunit center as in Tab. 6.2. If the force-distance curves exhibited more than one force peak, the first yielding force is depicted, and the largest yielding force determined from the largest peak of the adjacent maxima is given in brackets.

\begin{tabular}{|c||c|c|c|c|}
\hline & RHINOVIRUS & WITHOUT ACID & WITHOUT ZN & WITHOUT VP4 \\
\hline \hline 5-fold axis & $2.00(3.96)$ & $1.74(4.05)$ & $1.93(4.20)$ & 3.46 \\
3-fold axis & $1.38(1.75)$ & 1.58 & $1.28(1.62)$ & $1.35(1.71)$ \\
2-fold axis & 3.70 & 3.49 & 3.58 & 3.35 \\
subunit center & 3.54 & 3.53 & 3.67 & 3.86 \\
\hline
\end{tabular}


From the force-probe simulations in which the tip-sphere was pushed along the 5and 3-fold symmetry axes, four peaks with four yielding forces were obtained (see chapter 6.3.5). Two exceptions were found for the grid point at the pentamer center of HRV 16 without protein VP4 (yielding force: $3.46 \mathrm{nN}$ ) and the hexamer center of the capsid without fatty acid $(1.58 \mathrm{nN})$; their force-distance curves exhibited only one force peak.

The distribution of the yielding forces obtained at the 2-fold symmetry axes and the subunit centers was found to be homogeneous. The values were in the range of $3.35 \mathrm{nN}$ to $3.70 \mathrm{nN}$ for the pentamer center and between $3.53 \mathrm{nN}$ and $3.86 \mathrm{nN}$ at the subunit center for all four simulation systems. The hexamer centers exhibited the smallest yielding forces with values between $1.28 \mathrm{nN}$ and $1.58 \mathrm{nN}$ for the first force peaks in the force-distance plots and between $1.62 \mathrm{nN}$ and $1.75 \mathrm{nN}$ for the largest force peaks.

The forces seen for the first peaks in the force-distance plots obtained from pentamer indentations of the HRV 16 and the mutation capsids varied between $1.74 \mathrm{nN}$ and $3.46 \mathrm{nN}$ (only one peak was found for the capsid without VP4). The adjacent force peaks revealed the largest yielding forces between $3.96 \mathrm{nN}$ and $4.20 \mathrm{nN}$.

In summary, the smallest yielding force was determined at the 3-fold symmetry axis of the viral shell without zinc ions $(1.28 \mathrm{nN}$, first peak), the largest value was also found for this capsid at the pentamer center $(4.20 \mathrm{nN}$, second peak).

The estimated standard deviation, determined from all force-probe simulations, was in the range of $0.09-0.32 \mathrm{nN}$.

\subsubsection{Structural changes during deformation}

Besides the elastic constants and yielding forces presented above, two indentation simulations and the resulting force-time and force-distance plots are discussed in structural detail below. First, we focus on the force-probe simulation in which the tip-sphere was pushed from outside along the 2-fold symmetry axis of the complete HRV 16 capsid. Second, the results from the indentation of the pentamer center of the same capsid are described. The size of the deformed capsid area was obtained for both simulations, as well as the transition from elastic to plastic behavior.

\section{Deformation along the 2-fold symmetry axis}

The force-distance and force-time curve obtained from the indentation of the grid point at the 2-fold symmetry axis are shown in Fig. 6.21. A pre-linear (1) and a linear regime (2) are seen, whereas the rearrangement regime (3) is hardly pronounced and the linear force increase leveled off at a maximum force within a few picoseconds (650 ps-700 ps). 


\subsection{Results}

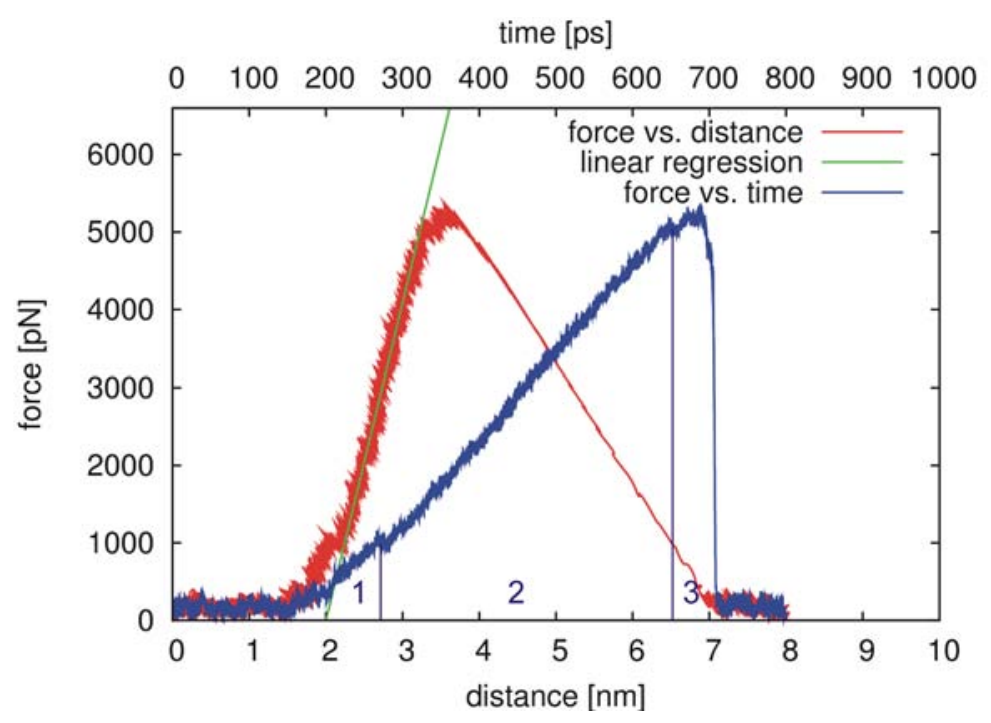

Figure 6.21: Force-distance (red) and force-time (blue) curves obtained from an indentation along the 2-fold symmetry of the HRV 16 capsid as shown in Fig. 6.10.

As a first step, the rmsd of the two subunits (SU) 33 and 35 that surround the 2-fold symmetry axis (Fig. 6.24), was determined, as well as the rmsd of two representative nearest neighbor subunits 20 and 21 (see e.g. Fig. 6.11) and the next nearest neighbor subunit 24 (Fig. 6.22).

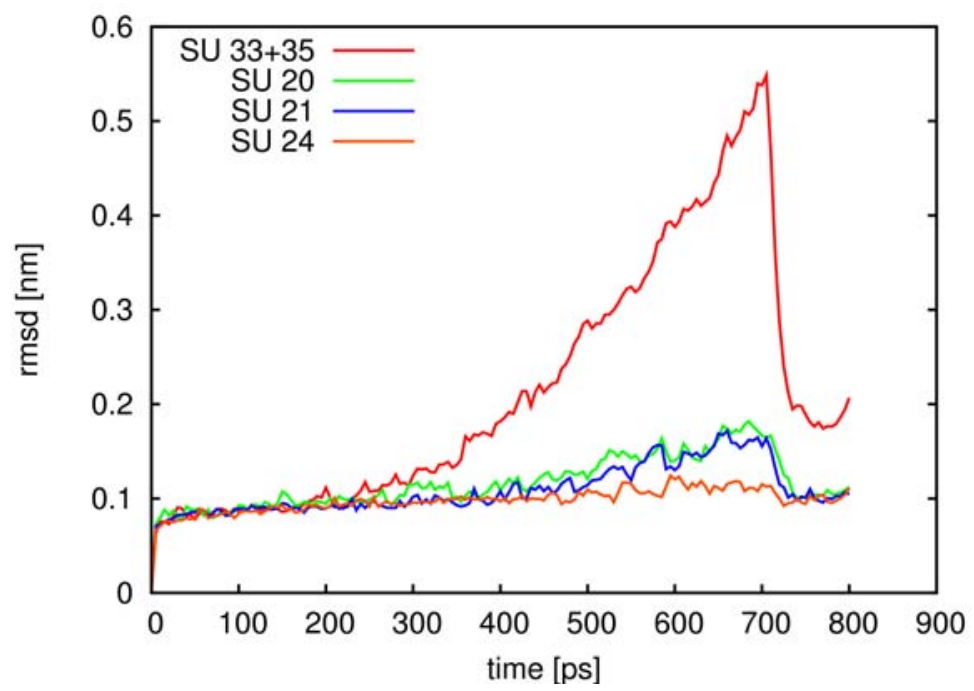

Figure 6.22: Rmsd of subunit (SU) 33+35 that are in direct contact with the 2-fold symmetry axis along with the tip-sphere was pushed during the FP simulation. The rmsd of the nearest neighbor subunits 20, 21 and next nearest neighbor subunit 24 are depicted as well. All rmsd were fitted to the initial structure of the complete HRV 16 capsid. 
When the tip-sphere approached the capsid, the rmsd of SU 33+35 started to increase slightly. The transition from the pre-linear to the linear regime at $280 \mathrm{ps}$ in Fig. 6.21 is represented in the rmsd by a larger rmsd increase that yielded a maximum rmsd of $0.55 \mathrm{~nm}$ at $700 \mathrm{ps}$ when the yielding force was reached. The deformation behavior of SU 33+35 is depicted in Fig. 6.24. As can be seen, the strongest deformations caused by the tip-sphere were located close to the indentation position. During deformation times in the linear regime, the $\beta$-sheet region of SU $33+35$ deformed only slightly (see $500 \mathrm{ps}$ in Fig. 6.24), whereas stronger deviations from the initial positions were seen for the protein loops due to thermal fluctuations additional to the indentation deformation. When the force dropped in the forcetime curve and the rmsd of the SU 33+35 decreased after $700 \mathrm{ps}$, the tip-sphere was pushed inside the viral shell and left the capsid within $10 \mathrm{ps}$ (see force drop in Fig. $6.21)$.

The nearest neighbor subunits 20 and 21 were deformed only weakly (Fig. 6.22). A maximum rmsd of $0.18 \mathrm{~nm}$ was determined, whereas the rmsd caused by thermal fluctuations was already of the order of $0.10 \mathrm{~nm}$. The rmsd of the next nearest neighbor SU 24 did not change during indentation with the tip-sphere.

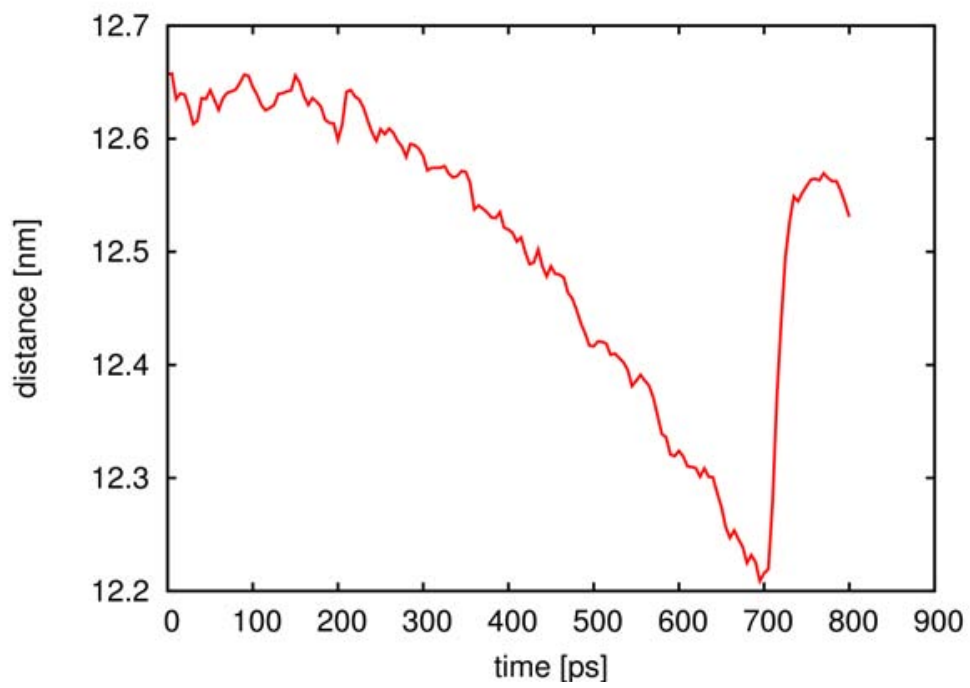

Figure 6.23: Distance change between the COM of SU 33+35 and the viral center during indentation along the 2-fold symmetry axis.

Fig. 6.23 depicts the distance change between the center of mass (COM) of SU $33+35$ to the capsid center during indentation. A reduced distance of $0.45 \mathrm{~nm}$ was found at the yielding point $(700 \mathrm{ps})$. Together with the deformation behavior of SU 33+35 as shown in Fig. 6.22 and 6.24, a volume decrease inside the capsid of $13.1 \mathrm{~nm}^{3}$ was estimated. No water flux through the shell was observed during indentation. Thus, compression of the bulk water inside the viral shell resulted in an increased internal capsid pressure of 17.9 bar which caused a force acting on the inner surface of subunit 33+35 of $232 \mathrm{pN}$ (surface area of SU 33+35: $A=129.8 \mathrm{~nm}^{2}$ ). 


\subsection{Results}

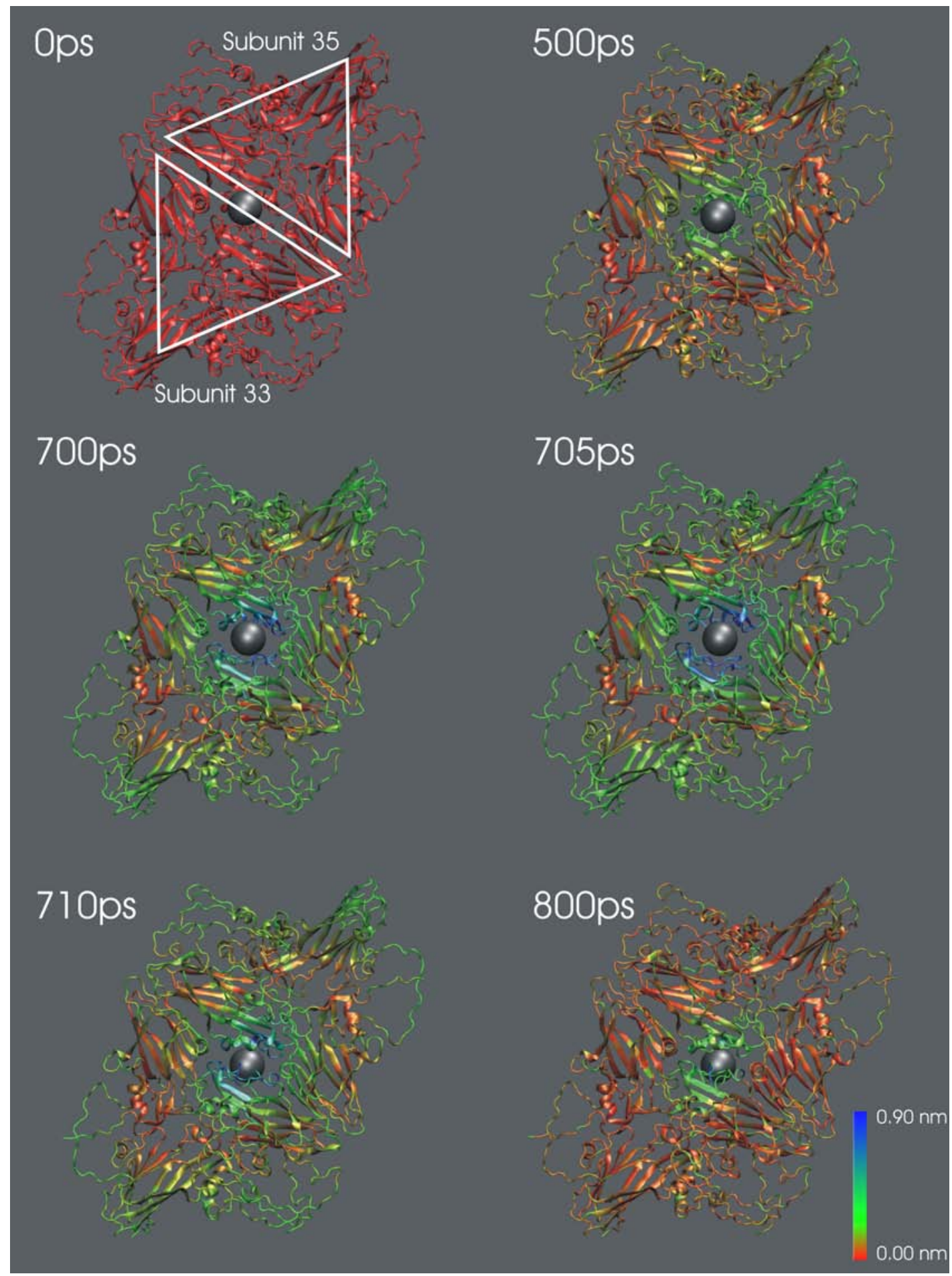

Figure 6.24: Snapshots from the simulation in which the tip-sphere (grey sphere) was pushing along the 2-fold symmetry axis at the middle of the interface of SU 33+35. Subunit 33 and 35 are marked with the white triangles. The deformation of each residue is color-coded as in Fig. 5.6. Yielding was obtained at $700 \mathrm{ps}$, whereas strong deformation of single residues was seen when the tip-sphere left the shell between 700-710 ps. 
In the following, the subunits except SU 33+35 were not considered for the investigations of deformation behavior because their structure hardly changed during indentation. As can be seen in Fig. 6.22, the rmsd of SU 33+35 did not return to its initial level after removal of the tip-sphere from the capsid (rmsd: $0.2 \mathrm{~nm}$ at $800 \mathrm{ps}$ in Fig. 6.22), suggesting a plastic deformation of this viral shell area. In the next step, the transition from elastic to plastic deformation of SU 33+35 was determined. Thus, the force-probe simulation was stopped at different times (see legend in Fig. 6.25). Subsequently the system was equilibrated with free MD simulations in which the harmonic potential acting on the tip-sphere was switched off.

The rmsd of SU 33+35 and the relaxation rmsd curves fitted on the starting structure of the virus before indentation are given in Fig. 6.25. The rmsd curves obtained from the relaxation simulations fall into two classes: (1) The rmsd curves that (nearly) returned to the initial level when external forces were switched off (colored curves at $690 \mathrm{ps}$ and below in Fig. 6.25), and (2) the curve that did not return to the level of thermal fluctuations (orange curve in Fig. 6.25). The first group reflects elastic deformation of SU 33+35 because the deformation was fully reversible. In contrast, plastic deformation occurred at the yielding point at $700 \mathrm{ps}$. Here the capsid structure did not completely recover, whereby the deformation was mainly caused by plastic deformation of the area close to the indentation position (Fig. 6.24, $800 \mathrm{ps).}$

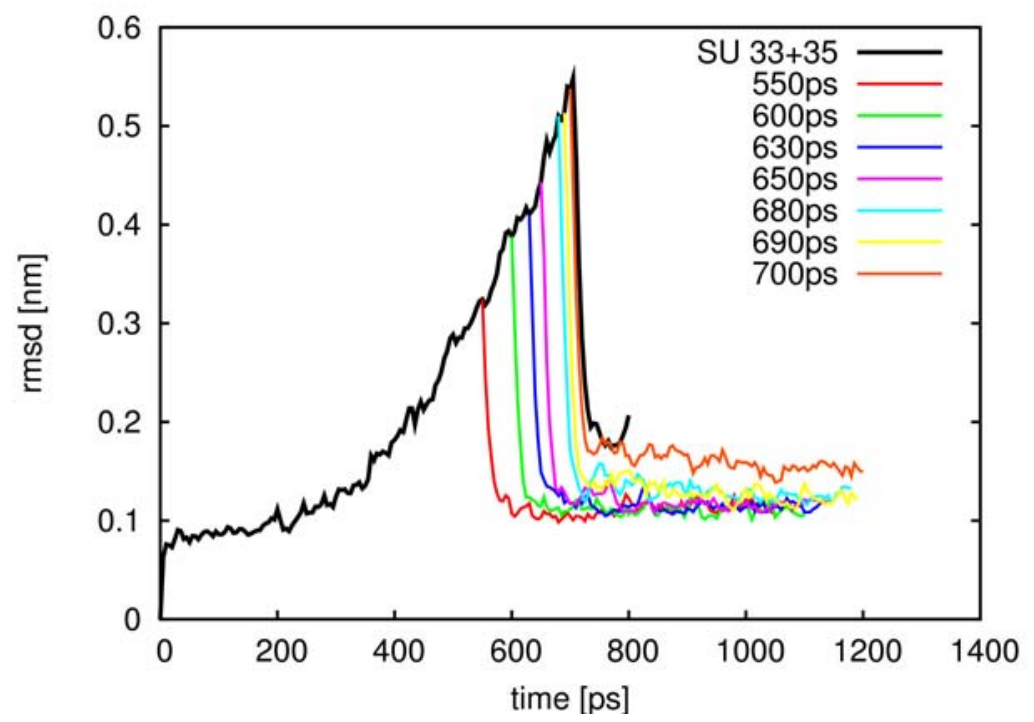

Figure 6.25: Rmsd of SU 33+35 during indentation (black curve) as shown in Fig. 6.22. Indentation was stopped at different times (see legend) and restarted with unrestrained tip-sphere. During the subsequent relaxation processes, the rmsd of SU $33+35$ is shown in color. Plastic deformation was observed at $700 \mathrm{ps}$, obtained from the increasing rmsd during the relaxation of the system.

To summarize, the force-distance and force-time curves obtained from the indentation along the 2 -fold symmetry axis show that the rearrangement regime was weakly 


\subsection{Results}

pronounced for this deformation. The transition from elastic to plastic deformation of SU $33+35$ took place at the yielding point $(700 \mathrm{ps})$. The remaining viral shell did not deform during the force-probe simulation because elastic and plastic deformation was restrained to the size of the subunits 33 and 35 that surround the 2-fold symmetry axis. 


\section{Deformation along the 5-fold symmetry axis}

Besides the typical force-distance plots that exhibit three deformation regimes as described before, several FP simulations in which the tip-sphere was pushed from the capsid outside and inside against the pentamer center and from the inside against the hexamer center, showed a different deformation behavior as depicted in Fig. 6.26a. In the force-probe simulation described here, the complete HRV 16 capsid was indented from outside along the 5 -fold symmetry axis through the pentamer center.

When the tip-sphere was pushed through the water towards the viral shell, the force started to increase when the tip-sphere came into contact with the outer protein layers of the capsid (pre-linear regime (1) in Fig. 6.26a). The transition to the linear regime (2) was observed at $220 \mathrm{ps}$ and the cross-over to the rearrangement regime at $270 \mathrm{ps}$ which yielded a maximum force at $330 \mathrm{ps}$. A subsequent force drop (330-350 ps) was recorded, followed by a second force increase (350-500 ps). Between $350 \mathrm{ps}$ and $500 \mathrm{ps}$, the three deformation regimes (1', 2' and 3' in Fig. 6.26) were seen as well. In total, the force-time and force-distance curves featured four such force increases and peaks, each containing the three regimes of deformation (see also Fig 6.29 to 6.32 ).

For all force-probe simulations in which the tip-sphere was pushed through the pentamer centers of the HRV 16 capsids and the three mutants we found that the elastic constants determined from the second and third linear regime were always similar, as well as the yielding forces of the second and third force peak. The elastic constants and yielding forces belonging to the fourth force increase usually exhibited smaller values than seen for the previous force regimes. Peak 4 did not occur in force-probe simulations of the HRV 16 capsid without protein VP4. Five of these proteins surround the 5-fold symmetry axis on the inner capsid surface (Fig. 6.31 and 6.32), and their removal shortened the indentation depth to $7.5 \mathrm{~nm}$ (compare Fig. 6.26a) before the tip-sphere left the capsid.

The indentation simulation of the pentamer center showed that capsid deformation was localized to the area of a pentamer (Fig. 6.29, 6.30). In order to study to what extent the pentamer and its proteins VP1 and VP4 which both surround the 5-fold symmetry axis, were deformed during penetration, the rmsd of the pentamer and proteins VP1 and VP4 surrounding the pentamer center compared to the initial structure of the complete viral shell was calculated (Fig. 6.26b).

Before the tip-sphere touched the capsid, the pentamer and VP1 rmsd curves leveled off at $0.1 \mathrm{~nm}$ due to thermal fluctuations. While the first two force peaks were reached at $330 \mathrm{ps}$ and $500 \mathrm{ps}$, respectively, the pentamer rmsd increased only slightly by $0.4 \mathrm{~nm}$ to $0.13 \mathrm{~nm}$ because only the outer protein loops of VP1 surrounding the pentamer center were deformed (increase of VP1 rmsd to $0.15 \mathrm{~nm}$ ). Subsequently during the third and fourth force increase, the rmsd increased to $2.6 \mathrm{~nm}$ at $980 \mathrm{~ns}$ after the tip-sphere had left the shell. The rmsd of protein VP1 behaved similarly to the pentamer rmsd until 780 ps. For later simulation times, the tip-sphere reached 


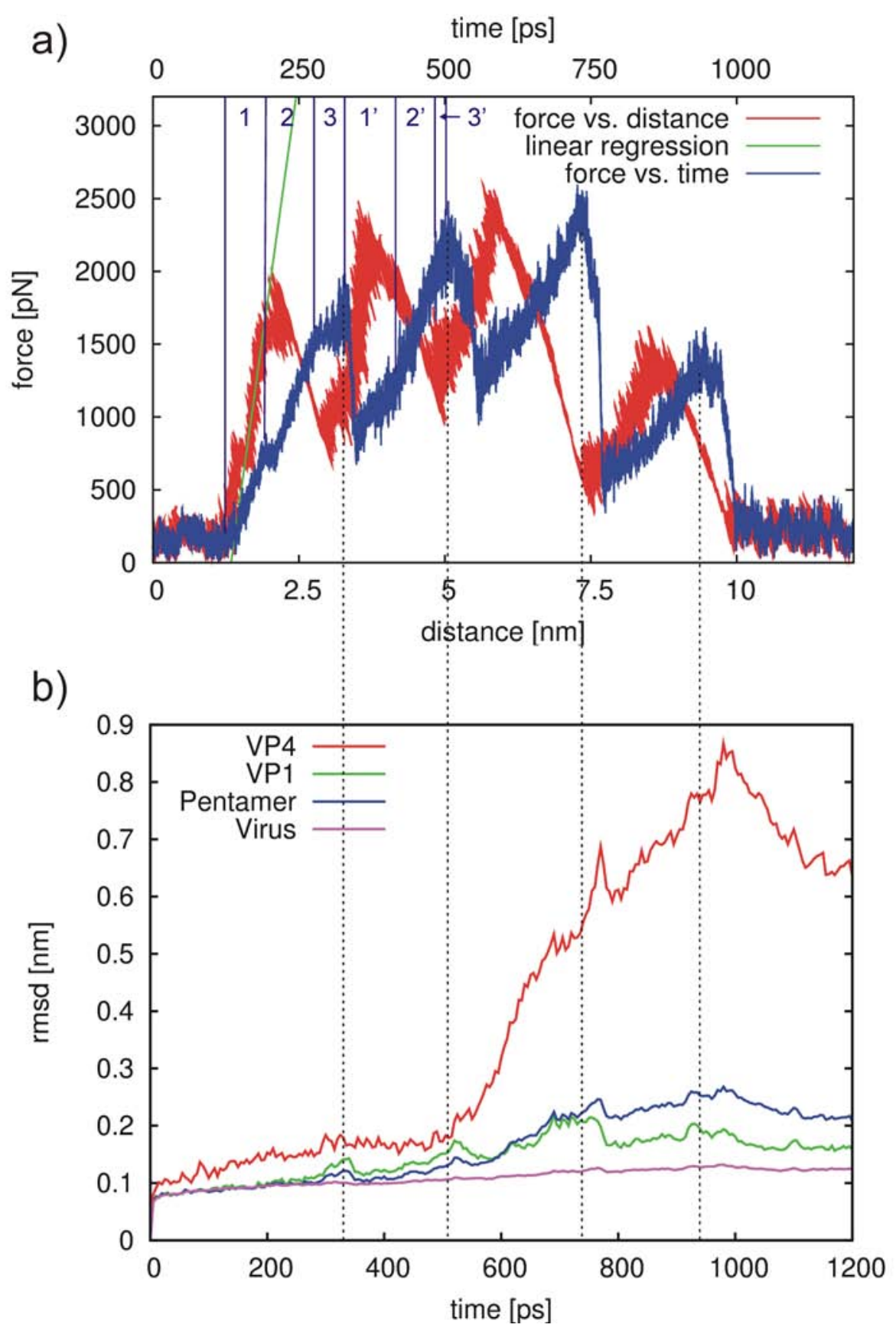

Figure 6.26: a) Force-distance and force-time curve obtained from indentation along the 5 -fold symmetry axis. The pre-linear (1, 1'), linear $\left(2,2^{\prime}\right)$ and the rearrangement regimes $\left(3,3^{\prime}\right)$ are marked, as well as the slope of the first linear regime (green line). b) Rmsd curves obtained from the same simulation for protein VP4, VP1, the complete pentamer and the viral capsid. All rmsd were fitted to the initial capsid structure before deformation. 
an indentation depth at which the tip-sphere came out of contact with the amino acids of protein VP1. At that point the tip-sphere entered the channel formed by five VP4 proteins around the 5-fold symmetry axis.

Protein VP4, which is located on the inner capsid surface around the 5-fold symmetry axis, showed the largest deformation during indentation with the tip-sphere. After the first two force peaks were passed at $500 \mathrm{ps}$, the tip-sphere approached the amino acids of VP4, and their rmsd rose to a maximum of $0.88 \mathrm{~nm}$ before the tipsphere left the shell at $980 \mathrm{ps.} \mathrm{Although} \mathrm{protein} \mathrm{VP1} \mathrm{surrounds} \mathrm{the} \mathrm{pentamer} \mathrm{center}$ on the outer capsid surface, its rmsd reached a maximum at $0.21 \mathrm{~nm}$, much smaller than the values seen for VP4 on the inner surface. Protein VP1 mainly consists of amino acids folded in a $\beta$-sheet. In addition, it is surrounded by proteins VP2 and VP3 which stabilize the structure. In contrast, the amino acids of VP4 are arranged in flexible loops moving freely in the bulk water inside the capsid (Fig. 6.31) which resulted in an increased equilibrium rmsd of $0.18 \mathrm{~nm}$ before the tip-sphere approached the capsid.

This flexibility is also reflected in the VP4 rmsd curve during the force drop before the tip-sphere left the shell (940-980 ps). Here, the tip-sphere was still inside the channel of the VP4 proteins, but only a small force was needed to push the tip-sphere along the channel out of the capsid due to the high mobility of the single residues. The maximum rmsd was therefore seen as the tip-sphere came out of contact with VP4 amino acids (0.88 $\mathrm{nm}$ at $980 \mathrm{ps}$ ) instead of at the yielding point (940 ps).

To summarize, the rmsd obtained for the pentamer was mainly determined by the rmsd of VP1 for simulation times until 780 ps. Subsequently, the strong deformation of $\mathrm{VP} 4$ resulted in a stronger increase of the pentamer rmsd compared to the rmsd of VP1.

The distance between the center of mass $(\mathrm{COM})$ of the indented pentamer and the capsid center was calculated (Fig. 6.27). During deformation of the capsid, the pentamer COM approached the viral center by $0.15 \mathrm{~nm}$, much less than the COM of the two subunits 33 and 35 during indentation along the 2-fold symmetry axis (Fig. 6.23). Nevertheless, the force acting on the inner pentamer surface was of the same order (approx. $210 \mathrm{pN}$ ) due to the increased internal water pressure. In contrast to the indentation along the 2-fold symmetry axis, here an increased area of deformation was seen which was about the size of a pentamer when the tip-sphere was pushed along the 5 -fold symmetry axis. 


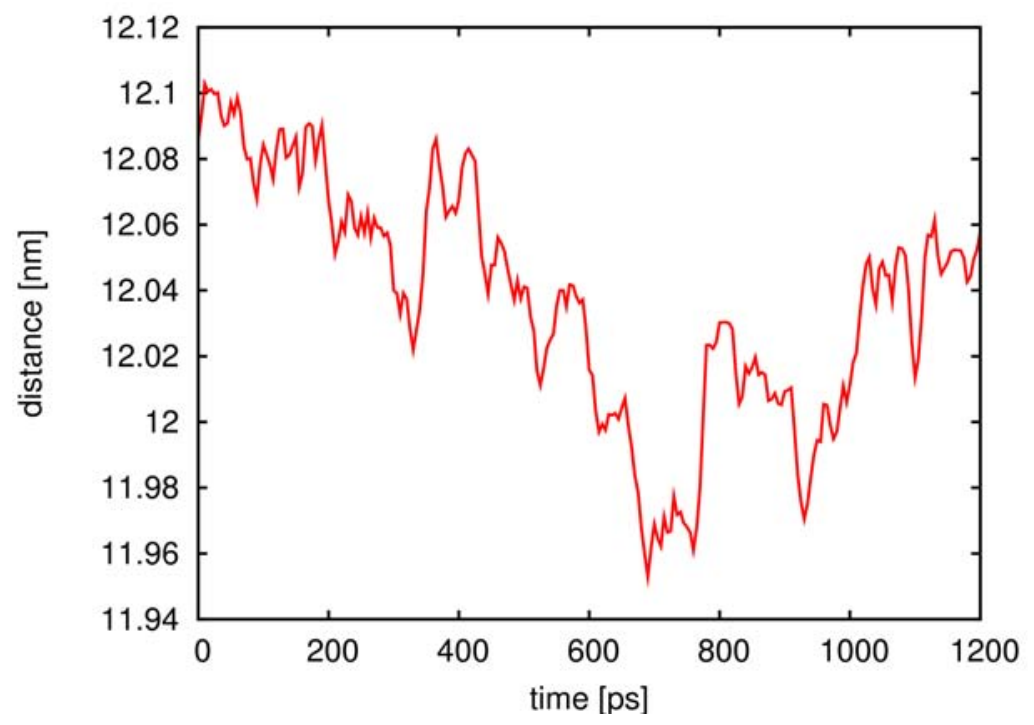

Figure 6.27: Distance change between the COM of the deformed pentamer and the viral center during indentation along the 5 -fold symmetry axis.

The rmsd curves shown in Fig. 6.26 depict a plastic deformation of the pentamer and protein VP1 and VP4 because the rmsd did not fully recover after the removal of the tip-sphere from the viral shell after 1000 ps. To investigate the transition from elastic to plastic behavior, the FP simulation was stopped at several time steps with subsequent relaxation of the system as described above. The obtained relaxation rmsd curves with respect to the initial capsid structure are shown in Fig. 6.28 for the pentamer and protein VP4. As found before from indentations along the 2-fold symmetry axis, the rmsd curves fall into two groups: The pentamer behaved elastically during indentation at 320 ps and below, whereas plastic behavior was already seen at $500 \mathrm{ps}$ and for later indentation times (Fig. 6.28a). Although the first yielding force was determined at approx. $320 \mathrm{ps}$, the pentamer structure (nearly) fully recovered after indentation. For the adjacent three linear regimes, fully elastic deformation of the capsid was not observed. Deformation was always superimposed by a plastic part, depicted by the offset of the relaxation rmsd curves to the level of thermal relaxation. Nevertheless, the elastic deformation obtained for all different simulation times, is seen as a drop in the rmsd curves as soon as the restraints acting on the tip-sphere were released.

Protein VP4 still deformed elastically at times when the deformation behavior of the complete pentamer was already plastic due to plastic deformation of single VP1 amino acids (550 ps in Fig. 6.28a,b). The transition to plastic behavior was obtained at $630 \mathrm{ps}$ (Fig. 6.28b). To summarize, the cross-over from elastic to plastic deformation of the pentamer and protein VP4 was proceeded after the indenting tip-sphere had overcome the first yielding force peak in the force-time curve. Plastic behavior was always superimposed by elastic deformation which was also observed from indentations along the 2-fold symmetry axis. 

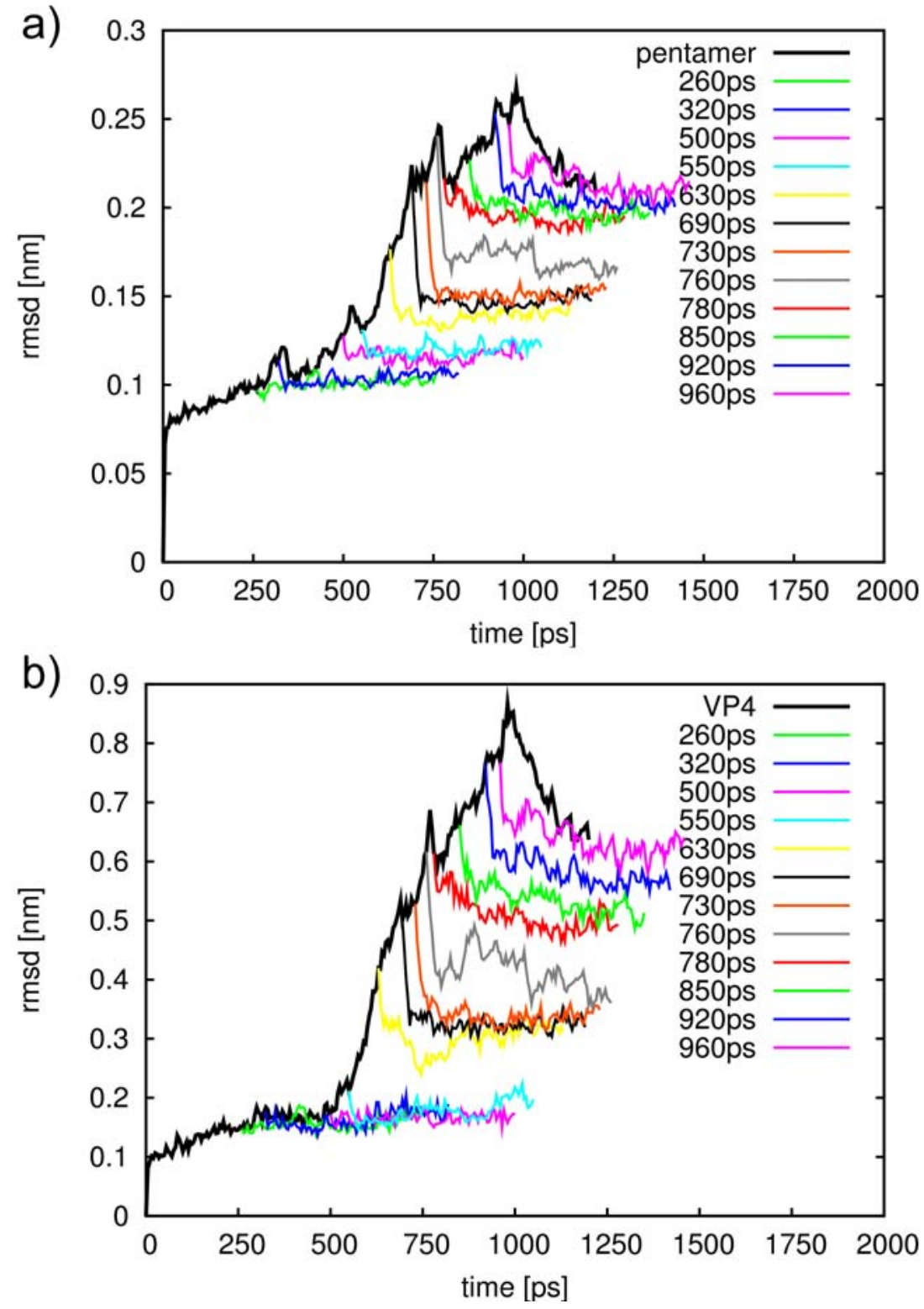

Figure 6.28: Rmsd of pentamer (a) and protein VP4 (b) (black curves) as shown in Fig. 6.25. The relaxation curves after release of the tip-sphere are shown in color. For the pentamer, plastic deformation started at 500 ps, obtained from the increasing rmsd during the relaxation of the system. Protein VP4 behaved plastically at 630 ps. 


\subsection{Results}

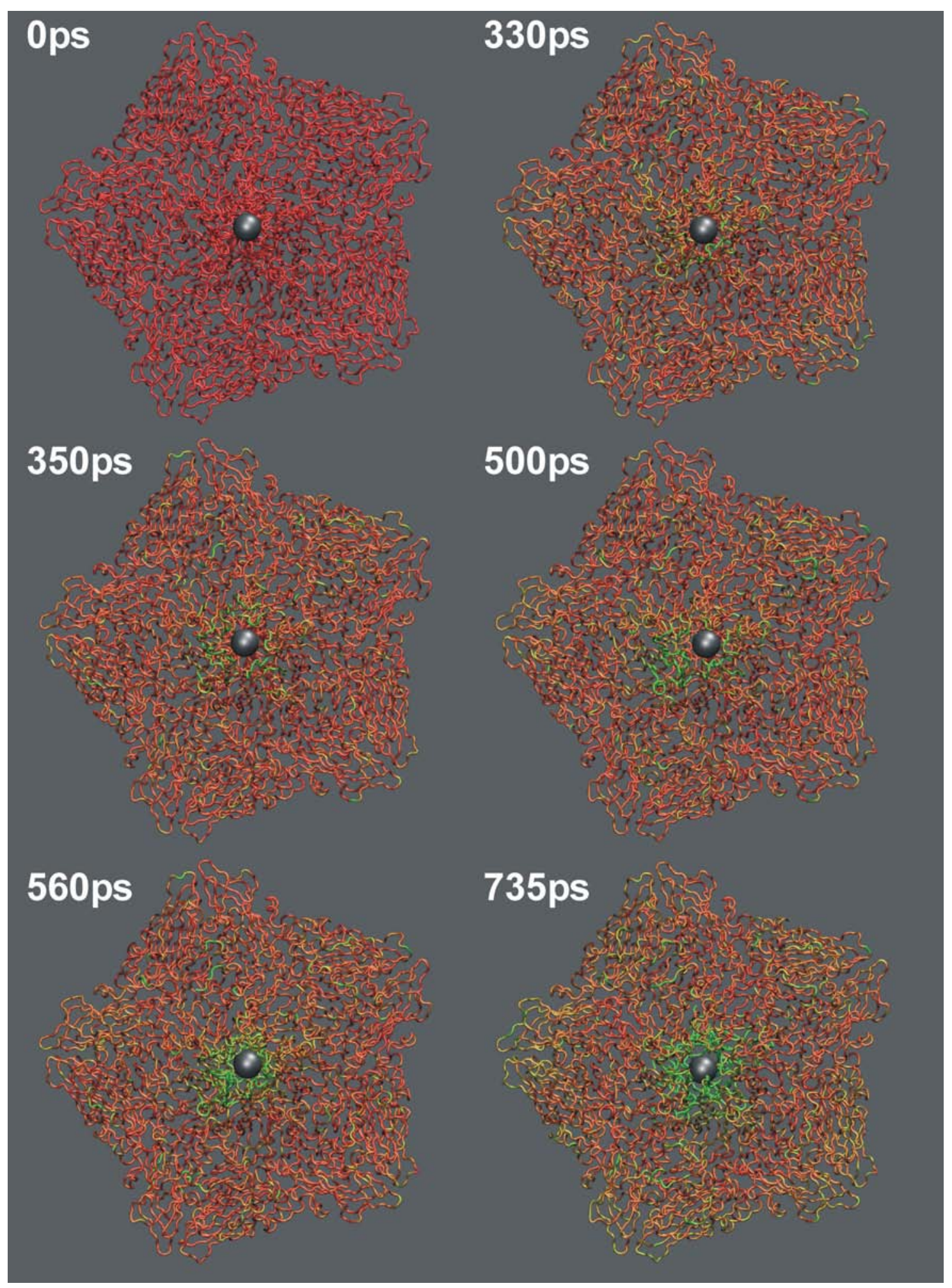

Figure 6.29: Snapshots of the pentamer (TOP-view) from the simulation in which the tip-sphere (grey sphere) was pushed from outside along the 5-fold symmetry axis. The deformation of each residue is color-coded as in Fig. 5.6. 
The mechanical properties of Human Rhinovirus 16

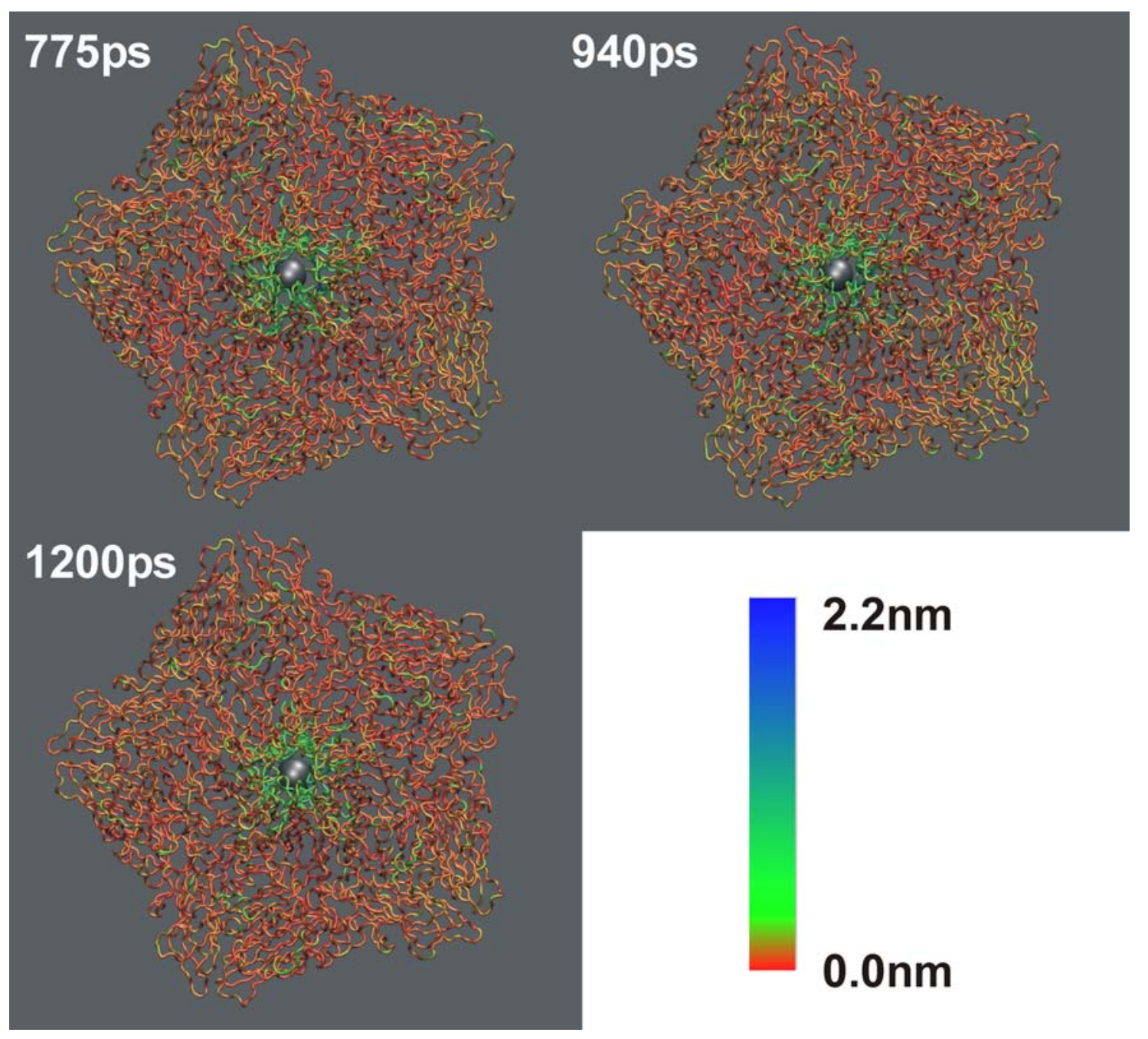

Figure 6.30: Snapshots of the pentamer as in Fig. 6.29. 


\subsection{Results}

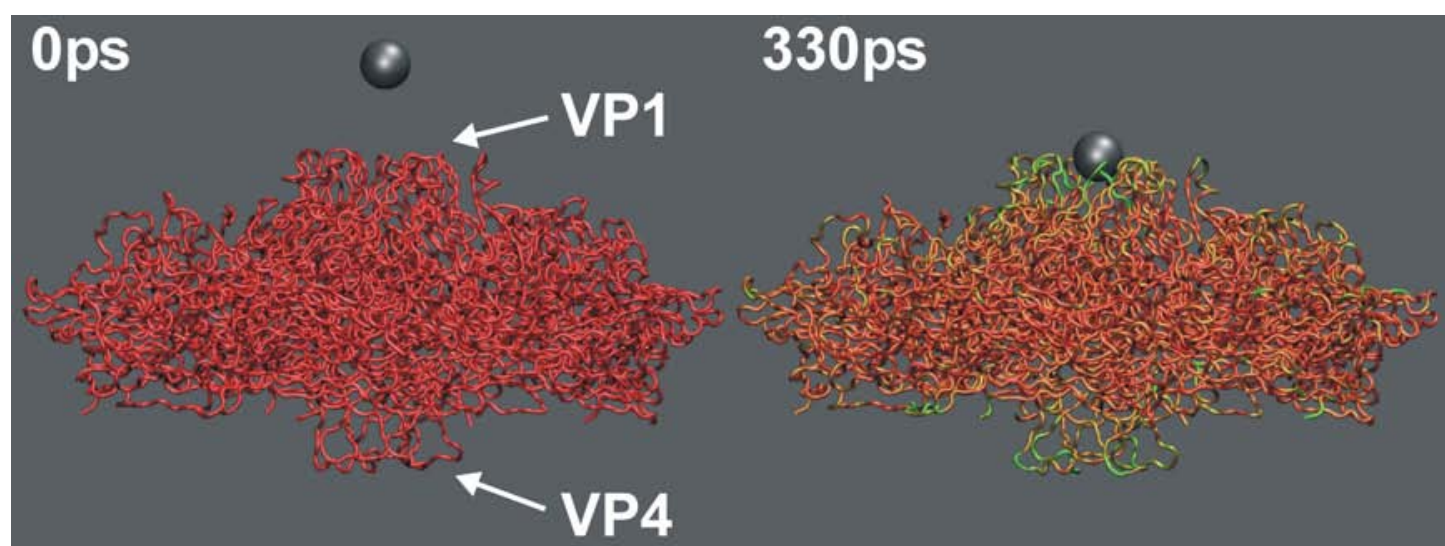

350 ps

500 s

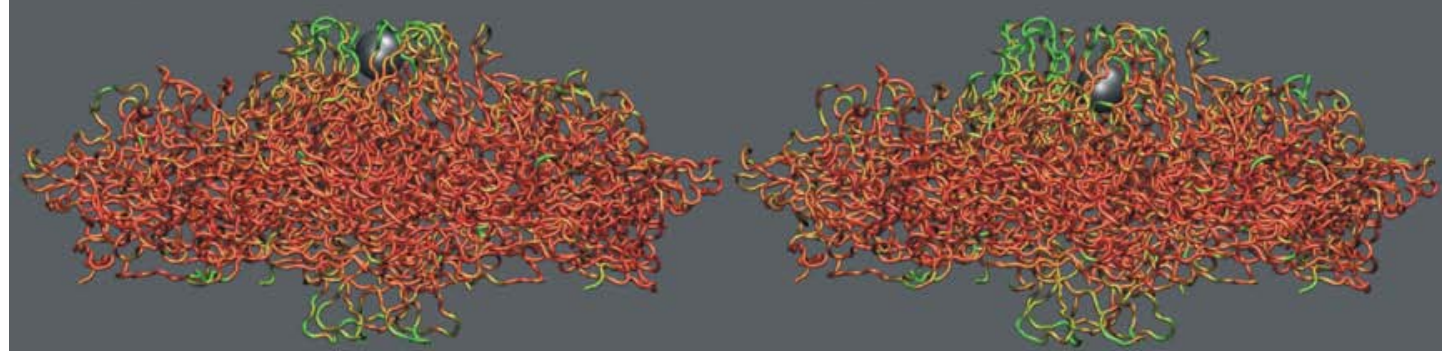

560 ps

$735 \mathrm{ps}$

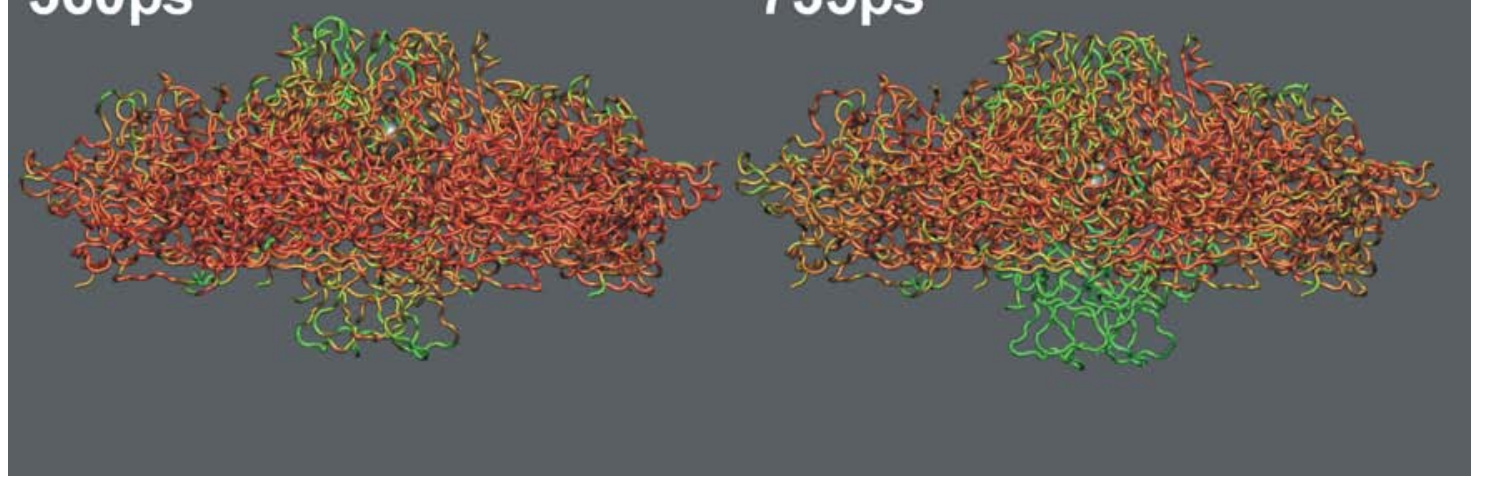

Figure 6.31: SIDE-View of pentamer shown as in Fig. 6.29. Loops of five VP1 proteins are located on the outer pentamer surface around the 5-fold symmetry axis. Five VP4 proteins surround the inner surface of the pentamer center and are depicted by the loops that form a channel along the 5-fold symmetry axis. 
The mechanical properties of Human Rhinovirus 16

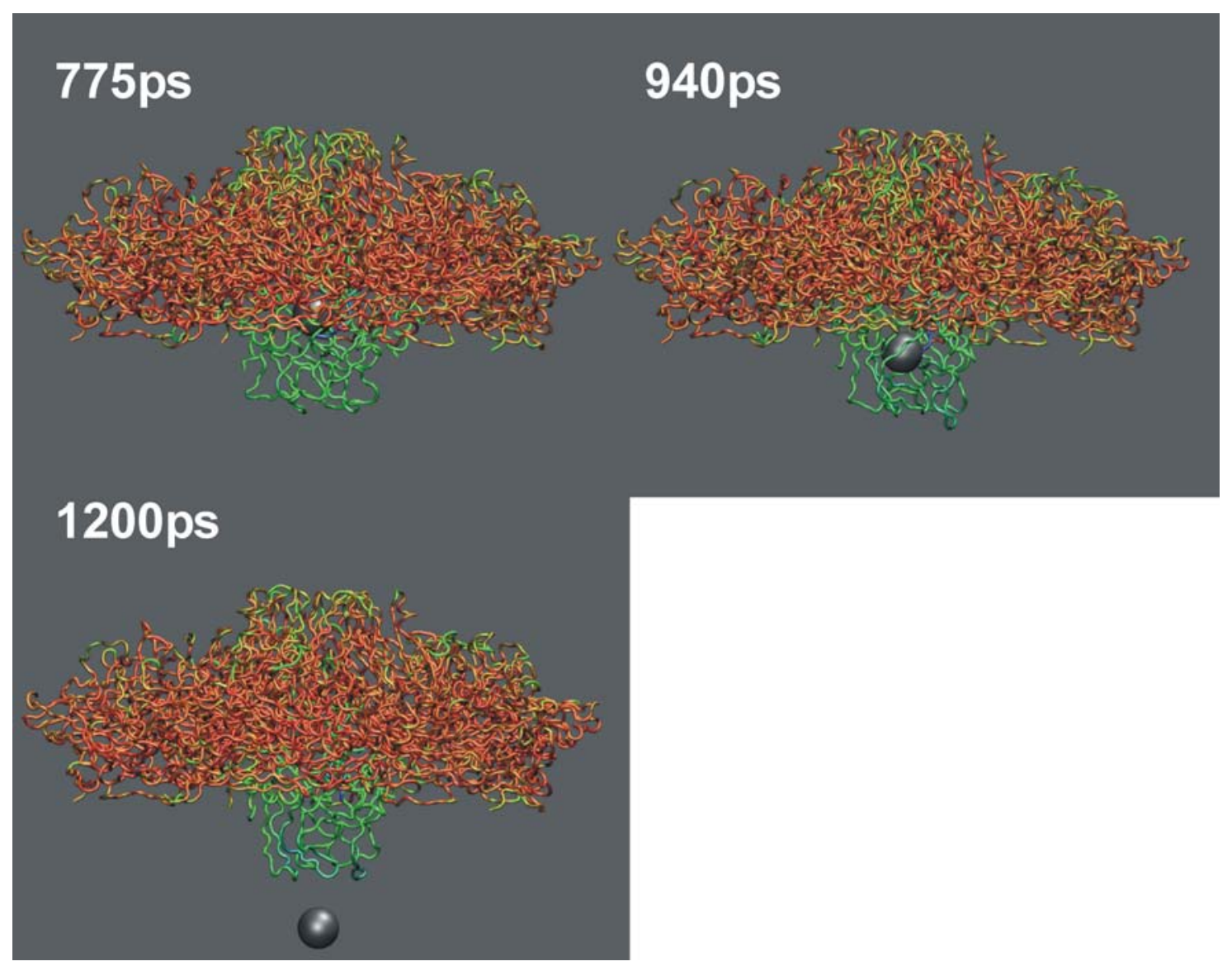

Figure 6.32: Snapshots of the pentamer as in Fig. 6.31. 


\subsection{Discussion}

\subsection{Discussion}

The mechanical properties of the Human Rhinovirus 16 capsids and three mutants of the capsid structure without the pocket factor, zinc ions, and protein VP4 were investigated in this chapter. In order to study the distribution of elastic constants and yielding forces on the viral capsid with the force-probe simulation technique, the four different viral shells were indented with a tip-sphere at 19 different grid point position on the outer capsid surfaces. Additionally, the tip-sphere was initially positioned inside the shell and subsequently pushed along the three different symmetry axes and against the subunit center towards the inner capsid surface. A total of $220 \mathrm{~ns}$ of simulation was performed.

The root mean square deviations between the HRV 16 structure and its three mutants after the equilibration phases were in the range of $1.3 \AA$ (HRV 16 - mutant without zinc) and $2.6 \AA$ (HRV 16 - mutant without VP4). All rmsd values between the structures were mainly caused by thermal fluctuations. The removal of VP4 protein increased the surface area of protein VP1 which came into contact with the water resulting in the largest rmsd between HRV 16 and the mutant without VP4. No significant differences and topological changes between the four capsid structures were observed.

Rossmann et al. proposed that the fatty acid inside the pocket in VP1 stabilizes the protein structure and a removal might cause a collapse of the structure, observed as a compactization of the protein or closing of the pocket [131,61]. This collaps was only observed when the ICAM-1 receptor was bound to the empty pocket $[162,61]$. For this reason it remains unclear whether the removal of the pocket factor without the attached receptor to protein VP1 results in a closing of the pocket or, alternatively, whether the receptor is necessary to induce a structural change. We suggest from our equilibration results of the complete HRV 16 capsid and the structure without the fatty acid, that a structural transition from the open to the closed state might be driven by the binding of the receptor to the VP1 protein. Nevertheless, this result has to be treated carefully. Because of the short equilibration simulations of $15.5 \mathrm{~ns}$, structural changes might take longer and occur at later times.

During all force-probe simulations, the force acting on the tip-sphere during indentation was monitored and force-distance and force-time curves were obtained. As already found from the force-probe simulations on Southern Bean Mosaic Virus (chapter 4, 5), three different deformation regimes of capsid indentation can be distinguished from these curves: a pre-linear regime of outer shell layer rearrangement, a linear regime of elastic shell deformation and a rearrangement regime which determines the transition from elastic to plastic deformation. From the slope of the linear regime, the elastic constant at the respective grid point was determined; the maximum force seen at the end of the rearrangement regime before fracture occurred depicted the yielding force.

The elastic constants and yielding forces on the outer capsid surface of HRV 16 and on that of the mutants were of the same order of magnitude (about $2 \mathrm{~N} / \mathrm{m}$ and 
$2 \mathrm{nN}$, respectively) as the values obtained for SBMV. We also found a heterogeneous distribution of mechanical properties, whereas their distribution on the rhinovirus surfaces varied with respect to the distribution on SBMV. Both capsids exhibit the softest position with the smallest elastic constant at the subunit centers. In contrast, the 5-fold symmetry axis, where the highest elastic constant was obtained for SBMV, showed the smallest value compared to the 3- and 2-fold symmetry axes that exhibited similar elastic constants. This result is in good agreement with the AFM experiments on full capsids of minute virus of mice by Carrasco et al. [44] who determined the smallest elastic constant at the pentamer center.

The distribution of elastic constants inside the subunit varied markedly between the two virus capsids of SBMV and HRV 16. The elastic constants inside SU 12 of SBMV did not show significant differences between the grid points at the protein centers and their interfaces. The values inside SU 35 of HRV 16 were in a range of $1.75-4.71 \mathrm{~N} / \mathrm{m}$, and the obtained values depended very much on the grid point positions; only small deviations of the grid point position could result in large changes of mechanical properties due to the very inhomogeneous protein structures.

After removal of the fatty acid, the zinc ions and the VP4 protein from the HRV 16 structure, the largest variations in elastic constants were seen along the symmetry axes, and at the subunit center which became stiffer as seen from the indentation results obtained for all mutants compared to the complete structure. The elastic constants found at the pentamer centers of all mutants increased slightly, but remained the softest symmetry axis. A large stiffness increase was seen at the 2 -fold symmetry axis after removal of the zinc ions. Nevertheless, marked changes in the elastic constants on the outer capsid surface, as obtained for SBMV after calcium removal, were not found for HRV 16 and the three mutants.

From the heterogeneous distribution of yielding forces on the outer capsid surface, the softest position was determined at the pentamer center. The most stable position was determined at the 2-fold symmetry axis, in contrast to yielding forces obtained for SBMV where the 2-fold symmetry axis exhibited the smallest yielding forces of all symmetry axes. The distribution inside the subunit was found to be very inhomogeneous, similar to the elastic constants discussed above. Also the removal of the pocket factor, the zinc ions, or VP4 mainly affected the yielding forces at the symmetry axes and the subunit center. The largest changes seen at the subunit center were of the order of $1 \mathrm{nN}$, whereas the overall distribution of yielding forces was hardly affected by the mutation of the capsid structure.

When the tip-sphere was pushed against the inner capsid surface, the smallest elastic constants and yielding forces were seen at the hexamer centers of all four capsids. The largest values were obtained at the pentamer center for both, elastic constants and yielding forces, whereas the force-distance curves determined from these simulations showed several elastic regimes and yielding forces (see below). Therefore, the largest elastic constants and yielding forces were always obtained from the second or third force increase in the respective force-distance plots. 


\subsection{Discussion}

After removal of the calcium ions from the capsid of SBMV, a dramatic change of mechanical properties was seen, which suggested a possible position on the viral shell that could break during viral infection and release the RNA. In contrast, a similar pathway cannot be inferred from the force-probe simulations on HRV 16 and the three mutants. Each capsid mutation alone did not result in a significant change of the elastic properties on the inner and outer capsid surfaces. Investigations on HRV 16 capsids with several mutations, e.g. the removal of the pocket factor and additionally the removal of VP4 of the same capsid could shed more light on structural changes needed for cell infection. It would be interesting to test if the ICAM-1 receptor could be modeled in the simulation system and docked to protein VP1 to cause a closing of the pocket. Furthermore, experiments showed that the loss of the VP4 protein is accompanied by an incubation of a receptor which was not modeled in our simulation $[176,177]$. Nevertheless, our simulations showed that the removal of protein VP4 and an emptying of the pocket alone do not result in a modified capsid structure which is able to release the RNA - at least at the relatively short time scales accessible to MD simulations. Hadfield et al. proposed that the zinc ions hold the VP1 proteins together, and their removal might cause a destabilization of the pentamer in the region around the 5-fold symmetry axis [61]. This effect could not be corroborated from our simulations. External influences like the docking of a receptor to the outer capsid surface might be neccessary to cause dematuration and is assumed to be the first step in cell infection.

Furthermore, two special force-distance and force-time curves were investigated. The first curves were obtained from a simulation in which the tip-sphere was pushed from the outside along the 2-fold symmetry axis. Three deformation regimes as discussed above were seen, whereas the rearrangement regime was very short and the linear regime directly reached up to the yielding point. Elastic deformations of the subunits surrounding the 2-fold symmetry axis were seen even a few picoseconds before the force peak was reached. Plastic deformation of the two subunits only occurred at the yielding point. In contrast, plastic deformation of the complete subunit was never seen in force-probe simulations of Southern Bean Mosaic Virus. Here, the subunit structures always recovered to their initial levels after penetration, and plastic deformation was only observed for single residues close to the indentation position. Propagation of the deformation on the capsid was restrained to the area of the subunits that directly touched the indentation position which was also found from the FP simulations of the SBMV capsid as well as for HRV 16.

The second force-time and force-distance curves described in detail were derived from indentation of the pentamer center. Also in these simulations, deformation was limited to the area of a pentamer, and adjacent subunits were not affected. The obtained force-distance curve exhibited four force peaks, each preceded by a pre-linear, linear and a rearrangement regime. The force fluctuations in all regimes were larger than those observed for indentations along the 2-fold symmetry axis. These large fluctuations were mainly caused by interactions between the tip-sphere and flexible loops of protein VP1 and VP4 that surround the pentamer center. 
While the first force peak was seen in the force-distance plot, the tip-sphere had not even touched the pentamer surface (330 ps in Fig. 6.31). Only single loops that surround and block the entrance of the 5-fold symmetry axis interacted with the tip-sphere and were finally pushed out of its way when the first yielding point was reached. The yielding forces obtained for peak two and three were always larger than for the first peak because the flexibility of the loops that were directly touched by the tip-sphere was reduced for deeper penetration, and additionally more residues were deformed during indentation. Nevertheless, indentation of the HRV 16 pentamer showed that the elastic constant obtained from the slope of the first linear regime in the force-distance curve was similar to the slope of the second and third linear regimes. Yielding force peak four was also smaller than the previous two peaks and only seen when protein VP4 was present in the simulation system. Five of these proteins surround the inner surface of the pentamer center. Its high flexibility resulted in smaller forces acting on the tip-sphere and larger deformations as seen in the rmsd curves of VP4 compared to the initial structures.

Although the first force peak was observed at $330 \mathrm{ps}$, the transition from elastic to plastic behavior of the pentamer was seen at the second force peak (500 ps). At the first peak, only deformations of the outer protein loops were obtained, the remaining pentamer structure and protein VP1 to which the loops belong remained nearly unaffected. Protein VP4 still behaved elastic until 630 ps because larger indentation depth were needed until the tip-sphere came into contact with VP4 and plastically deformed the protein. During the second to fourth linear regime in the force-distance plot, elastic deformation of the pentamer was seen, whereas the deformation was always superimposed by a plastic part. Both rmsd curves of the pentamer and VP4 let us suggest that plastic deformation got more and more pronounced with increasing indentation depth as depicted by the rising rmsd curves during the relaxation phases. The snapshots of the trajectory and the obtained deformation of each pentamer residue showed that the deformation only weakly propagated from its origin at the pentamer center along the pentamer surface to its borders to the next subunits. Only the deformation of single residues close to the tip-sphere position increased during indentation. The deeper the tip-sphere penetrated the pentamer, the larger the deformation of protein VP4 compared to the remaining proteins VP1-VP3 of the capsid.

Indentation along the 2- and 5-fold symmetry axes exhibited a pressure increase inside the capsid due to water compression and non-existing water flux through the shell. The resulting force from the water molecules acting on the area of deformation on the inner capsid surface was of the order of approx. $200 \mathrm{pN}$, roughly $10 \%$ of the yielding forces, as also found from force-probe simulations of SBMV.

To conclude, we found a higher elasticity of the capsid along the 2-fold symmetry axis with larger elastic constants and yielding forces as obtained along the pentamer center. The transition from elastic to plastic behavior occurred for larger indentation depth along the 2-fold symmetry axis than along the 5-fold symmetry axis.

Our simulations show that each structural mutation of the HRV 16 coat protein at 


\subsection{Discussion}

which the pocket factor, the zinc ions and protein VP4 were removed, did not cause a significant change in mechanical properties, and thus did not result in a structure that is able to release the RNA. We therefore suggest that other capsid "variants", a combination of several mutations on one capsid structure and/or additional binding of the ICAM-1 receptor to the pocket in VP1 is necessary to cause rupture of the shell and, thus, viral infection. Furthermore, interaction of the RNA with the coat protein might be essential in the process of dematuration as well. 


\section{Deformation of the globular titin kinase protein domain}

\subsection{Introduction}

Recent atomic force microscopy measurements on various viral shells have shown that these capsids behave highly elastic upon indentation with the AFM tip. Even indentations of $30 \%$ in capsid height do not cause rupture [43], and our force-probe simulations of the shell of Southern Bean Mosaic Virus and Human Rhinovirus 16 prove that high external forces are needed to cause local plastic deformation. The question arises, if this high elasticity is a special property of viral shells which is hardly found for other proteins [31].

To answer this question, we compared the elastic behavior of SBMV and HRV 16 with the deformation behavior of a non-viral titin kinase protein domain. To this end, force-probe simulations were performed similar to the ones described for the viral shells above. The protein was chosen because of its similarity to the viral coat proteins. In particular, the residues are folded to a $\beta$-sheet domain surrounded by loops, which can be considered as a similar structure of a $\beta$-barrel motif as found for SBMV and rhinovirus coat proteins.

\subsection{Methods}

The simulation system of the solvated globular titin-kinase protein domain (PDB code: 1tit) was set up and equilibrated for several nanoseconds by Ulf Hensen [139, 180]. To perform force-probe simulations on this protein, a tip-sphere of the same size as used for the simulations of SBMV and HRV 16, was placed in water close to the protein surface and pushed with a probe-velocity of $0.01 \mathrm{~nm} /$ ps against the $\beta$-sheet domain (Fig. 7.3). To avoid yielding of the whole protein when the tipsphere was pushed against the surface, four $\mathrm{C}_{\alpha}$ atoms on each of the four "protein corners" were fixed with position restraints. From the force acting on the tip-sphere during deformation and the resulting force-distance curve, the elastic constant of the $\beta$-sheet domain was determined, as well as the yielding force.

In order to study the deformation behavior of the protein, the simulation was stopped at different simulation times with subsequent equilibration simulations as described before from studies of SBMV and HRV 16 (chapter 5.3.4 and 6.3.5). Here no force was acting on the tip-sphere anymore and the system was relaxed for $500 \mathrm{ps}$. The root mean square deviation of the protein compared to its initial structure was 
monitored during deformation and relaxation.

\subsection{Results}

During deformation of the titin kinase protein domain with the tip-sphere, a forcetime and force-distance curve was obtained (Fig. 7.1).

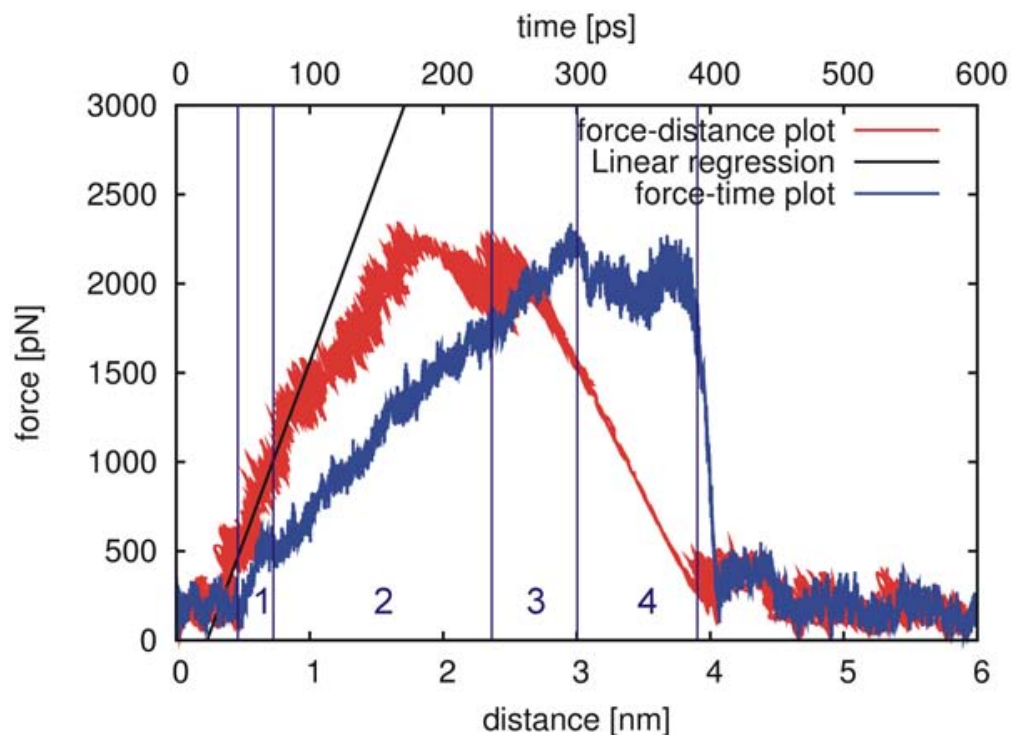

Figure 7.1: Force-distance plot (red) and force-time plot (blue) for pushing the tip-sphere with a velocity of $0.01 \mathrm{~nm} / \mathrm{ps}$ against the protein domain. The blue lines separates the four deformation regimes. The black line marks the slope of the linear regime from which the elastic constant was determined.

Similar to the force-probe simulations of the shell of SBMV and HRV 16, the force acting on the tip-sphere increased as the tip-sphere came into contact with the protein. The force continued to rise during indentation and leveled off at a maximum force before the tip-sphere left the protein. Fig. 7.1 depicts four deformation regimes in the force-time plot: (1) a pre-linear, (2) a linear, (3) a rearrangement and (4) a yielding regime. From the slope of the linear regime, an elastic constant of $2.0 \mathrm{~N} / \mathrm{m}$ was found. For higher indentations, the force yielded in a maximum of $2.26 \mathrm{nN}$ at a force plateau, the yielding regime (4), between $300 \mathrm{ps}$ and $390 \mathrm{ps}$. The indentation depth during this regime increased by $1.0 \mathrm{~nm}(1.7-2.7 \mathrm{~nm}$ in the force-distance plot, Fig. 7.1), followed by a force drop for even deeper indentations when the tip-sphere left the protein at $400 \mathrm{ps}$.

In order to investigate if the titin protein deforms elastically or plastically during deformation and if a transition between the different deformation behaviors could be observed, the force-probe simulation was stopped at different times (see Fig. 7.2 legend) and subsequently equilibrated for $500 \mathrm{ps}$ without restraints on the tip-sphere. 


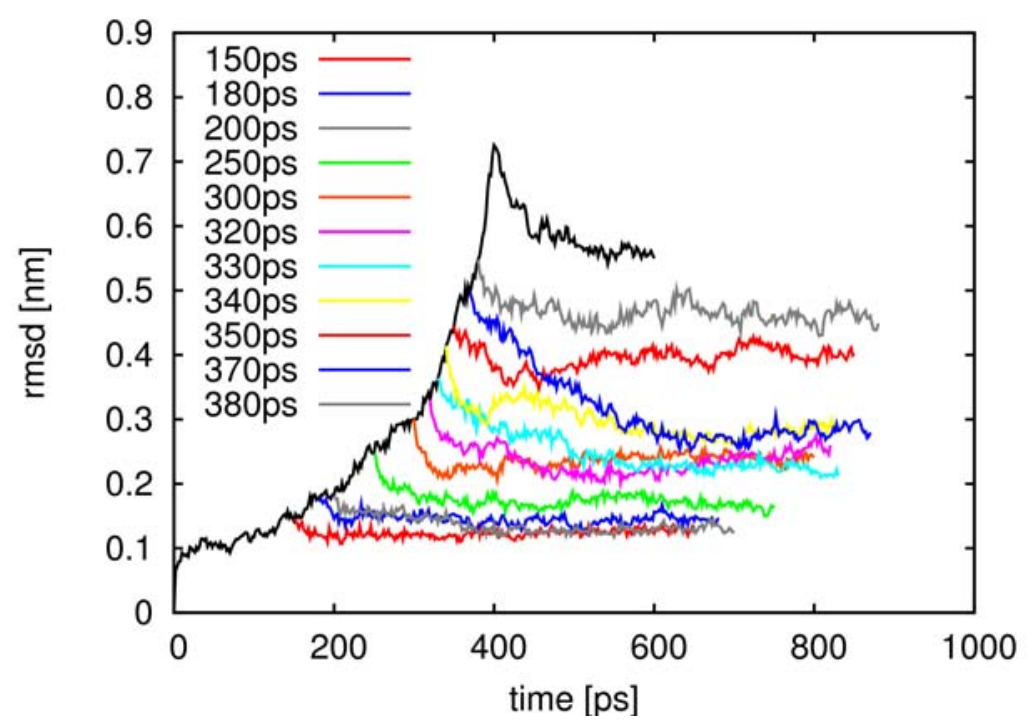

Figure 7.2: Rmsd of the titin kinase protein domain during the force-probe simulation (black curve). Indentation was stopped at different times (see legend) and restarted with unrestrained tip-sphere. During the subsequent relaxation processes, the relaxation rmsd curves are shown in color.

Before the tip-sphere touched the protein, the equilibrium rmsd of the titin domain was approx. $0.12 \mathrm{~nm}$ due to thermal fluctuations (0-50 ps in Fig. 7.1). The rmsd of the protein with respect to the initial structure increased to $0.75 \mathrm{~nm}$ during deformation until $400 \mathrm{ps}$, the end of the yielding regime. When the tip-sphere left the protein, the rmsd did not return to its initial value, suggesting a plastic deformation of the titin domain. Interestingly, the slope of the rmsd curve increased markedly during the yielding regime compared to the slope obtained for the linear and rearrangement regimes.

When the force-probe simulation was stopped at several times, the relaxation rmsd curves always declined during the first picoseconds. Until 200 ps when a linear increase in the force-time plot was obtained, the relaxation rmsd curves declined from approx. $0.18 \mathrm{~nm}$ to $0.12 \mathrm{~nm}$, the value of thermal relaxation, implicating elastic deformation of the protein in the linear force regime. Plastic deformation was observed for $250 \mathrm{ps}$ (green curve in Fig. 7.2) and later simulation times, obtained from the rmsd which was irreversible on time scales of the equilibration simulation. Here, plastic deformation got more and more pronounced with continuing penetration of the protein, as can be seen from the increasing relaxation rmsd curves. The strongest deformation in terms of the largest relaxation rmsd value was seen after yielding at $400 \mathrm{ps}$. When the tip-sphere left the shell after $400 \mathrm{ps}$, the rmsd leveled off at $0.56 \mathrm{~nm}$ (black curve in Fig. 7.2). Four snapshots of the force-probe simulation are shown in Fig. 7.3. 

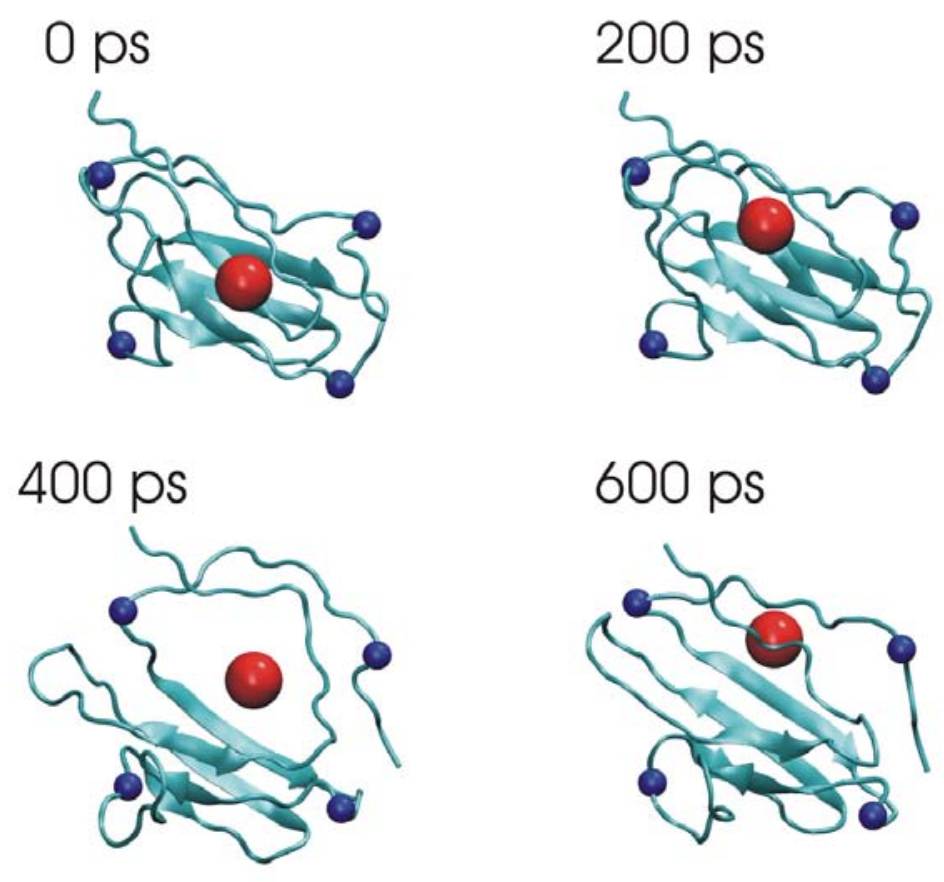

Figure 7.3: Sketch of the titin kinase protein domain (cyan) during deformation. Red sphere: tip-sphere, blue spheres: $\mathrm{C}_{\alpha}$ atoms which positions were restrained during deformation.

The $\beta$-sheet of the titin protein behaved stiff, as already found for the shell of SBMV. As can bee seen from Fig. 7.3, the tip-sphere was pushed aside to the more flexible loops when it tried to penetrate the $\beta$-sheet.

\subsection{Discussion}

From the force acting on the tip-sphere during the force-probe simulation, a forcedistance curve was determined from which an elastic constant and yielding force of the titin kinase protein at the indentation position were obtained. Both values were of the order of the determined elastic constants and yielding forces seen in the indentation simulations of the viral shells of SBMV and HRV 16. Differences between the results from the virus shell simulations and the titin protein occurred in the force-time and force distance plots (Fig. 5.1 for SBMV, 6.21 for HRV 16). All curves exhibited three deformation regimes: The pre-linear regime of single surface atom rearrangements when the tip-sphere came into contact with the protein, a linear regime of elastic deformation with a subsequent transition to the rearrangement regime that leveled in a yielding force. Only the force-time curve of the titin domain exhibited a fourth regime, the yielding regime, at which the yielding force remained stable in the range of $1.8 \mathrm{nN}$ and $2.2 \mathrm{nN}$ for $100 \mathrm{ps}$ before the force dropped when the 
tip-sphere left the protein. During deformation in the yielding regime, the indentation depth continued to increase by $1 \mathrm{~nm}$. In contrast, the maximum force seen in all viral shell indentation simulations was obtained as a peak in the force-time and force-distance curves, and a force plateau was never found. Thus, as soon as the yielding point was reached in the capsid simulations, deeper indentations beyond the yielding point immediately caused rupture of the area of indentation on the capsid resulting in a rapid force drop, and the tip-sphere was pushed out of the viral shell.

Further differences can be seen in the relaxation behavior when the restrained tipsphere was released. Whereas for the capsid simulations the deformation of the shell was still elastic in the rearrangement regime (Fig. 5.8 for SBMV, Fig.: 6.25 for HRV 16), a plastic behavior was already found for this regime for the titin domain deformation (250 ps in Fig. 7.2). Plastic deformation obtained as fracture behavior of the indentation position was always seen at the yielding point in the viral shell simulations, whereas the viral protein structure partially recovered from the deformation immediately after yielding when the tip-sphere came out of contact with the capsid. Plastic deformation of the titin domain obtained at the yielding force got larger with even deeper indentations during the subsequent yielding regime. The force remained constant for $90 \mathrm{ps}$, in contrast to the force drop and the recovery process of the structure subsequent to the yielding point as seen for the viral capsid simulations.

Viral shell proteins are always considered as being extremely elastic. We confirm this assumption with the observation that capsids still deform elastically in the rearrangement regime in which other proteins like the titin domain already behave plastically. Furthermore, a strong plastic deformation behavior was observed for a long range of simulation time and indentation depths in the yielding regime obtained from force-probe simulations on the titin domain which was never seen for SBMV and HRV 16.

Finally, it should be emphasized that the force-probe simulation on the titin kinase protein domain was one example of non-viral protein deformation with viral shell coat proteins, and further simulations would be necessary to corroborate our conclusions. 
7.4 Discussion 


\section{Summary and Conclusions}

We have studied the mechanical properties of the icosahedral shells of Southern Bean Mosaic Virus (SBMV) and Human Rhinovirus (HRV) 16 by extended all-atom forceprobe molecular dynamics simulations, covering a total time of nearly $0.5 \mu \mathrm{s}$. Both capsids were fully solvated in water on the protein surfaces. The simulation system of HRV 16, including approx. 800,000 water molecules, comprised over 4,200,000 atoms, the system of SBMV over 4,500,000 atoms, to our best knowledge the largest biomolecular simulation systems in the world.

The spatial distribution of elastic properties - quantified by elastic constants and yielding forces - on the inner and outer capsid surfaces were studied on atomic length scales with force-probe simulations. In order to obtain comparison with atomic force microscopy (AFM) experiments, a Lennard-Jones sphere, which served as a model of a small and very sharp AFM tip, was pushed with a constant velocity against and through the capsids of SBMV and HRV 16. A tight grid of 19 evenly distributed indentation positions on the internal and external capsid surfaces covered the complete subunit and the directions along the 5-, 3- and 2- fold symmetry axes. From the force acting on this tip-sphere, force-time and force-distance curves were obtained from each indentation simulation. Overall, three deformation regimes were distinguished: i) a pre-linear regime of outer capsid layer rearrangements when the tip-sphere approached the protein, ii) a linear regime of elastic capsid deformation, and iii) a sublinear rearrangement regime of larger force fluctuations that leveled in a yielding force. Subsequently, the tip-sphere left the shell for larger indentation depths, and a force drop was seen in the force-distance curve. The elastic constant of the capsid position the tip-sphere was pushed towards was determined from the slope of the linear regime. The maximum force in the force-distance plot defined the yielding force.

A heterogeneous distribution of elastic constants and yielding forces with values between 1.5-5.2 N/m and 1.3-5.1 nN, respectively, was found for the capsids of SBMV and HRV 16. Our simulations showed a weak stiffness and stability, obtained as small elastic constants and yielding forces, respectively, at the subunit center of the inner and outer capsid surfaces of both shells, SBMV and HRV 16. The least stiff position of the HRV 16 capsid was found at the inner and outer pentamer center, as well as the inner pentamer center of SBMV. In contrast, the pentamer center on the outer shell of SBMV exhibited the largest elastic constant. The most stiff and stable position on the capsid of HRV 16 was found along the 2-fold symmetry axis, as already proposed from AFM experiments on the $\mathrm{T}=3$ minute virus of mice [44]. Large elastic constants and yielding forces along the 2-fold symmetry axis of SBMV were only found when the capsid was indented from the inside. On the outer 
capsid surface, the 2-fold symmetry axis exhibited a small stiffness and stability. Such discrepancies between the elastic constants and yielding forces obtained on the same grid point position on the inner and outer surfaces were caused by the highly heterogeneous protein structure, mainly characterized by the arrangements of the $\beta$-strands.

The elastic properties of icosahedral viral shells with $\mathrm{T}=3$ symmetry have been studied before with coarse grained and continuum models in order to investigate if the mechanical properties of the different capsomer structures vary [50, 22, 49]. Contradicting results about the elastic constants of these pentamers and hexamers were published [31], whereas our force-probe simulation results give a clear answer to this question: Although the assembly of subunits for SBMV and HRV 16 subunits is very similar, both capsids exhibit very different mechanical properties. The stiffness of hexamers and pentamers varies and depends on details of the atomic structure. Therefore no general picture for the distribution of mechanical properties on capsids with $\mathrm{T}=3$ symmetry can be drawn. Our atomistic simulations clearly show that atomic detail cannot be neglected, because the atomic structure is a major determinant of the viral shell's mechanical properties, together with the geometrical arrangement of the subunits to an icosahedron.

The first step in viral infection of bean leaves with SBMV particles is the removal of the 180 calcium ions from the capsid structure [117-119, 60]. To study possible changes of the mechanical properties of SBMV without calcium compared to the coat protein with ions, $\mathrm{Ca}^{2+}$ ions were removed from the viral shell with subsequent equilibration of $32 \mathrm{~ns}$ with MD simulations. Our equilibration simulations show that the difference of the capsid structures with and without $\mathrm{Ca}^{2+}$, measured as the root mean square deviation between the structures, was small and of the order of thermal fluctuations. The reported swelling of the viral shell after calcium removal was not observed [120]. Three explanations seem to be likely for our findings. First, swelling was reported to occur in an alkaline environment at $\mathrm{pH}=7.5-8.25$ which was not present in our simulations. Second, swelling was observed to take three minutes until a volume increase of up to $44 \%$ was seen. Thus, the simulation time scale was much too short to corroborate the experimental observations. Third, interactions of the RNA with the coat protein lead to a swelling, whereas the genetic material was missing in our simulations.

After calcium removal force-probe simulations were performed in which the same 19 grid point positions on the inner and outer viral surface were indented as for the structure with ions. No significant change in mechanical properties was observed compared to the complete capsid with ions, with the exception of a marked softening along the 5-fold symmetry axis. Due to the fact that the pentamer center exhibited the highest stability when ions were present in the structure but became the least stable capsid position of the surface of SBMV without calcium, we suggest that dematuration and, thus, rupture might occur at the pentamer centers. Therefore the pentamer center might act as a possible port for RNA release after calcium removal. 
The effect of sufficient energy minimization and protein structure equilibration on mechanical properties was studied. Therefore, protein structures of SBMV with and without calcium were extracted from different equilibration times and used as starting structures for the force-probe simulations. It turned out that the obtained elastic constants and yielding forces for differently converged structures were very similar to the results discussed above. A fully converged protein structure could not be guaranteed after the equilibration simulations which might has an effect on capsid swelling after calcium removal as discussed above. Nevertheless, we obtained reliable results for the mechanical properties and the values of elastic constants and yielding forces.

The next systems studied with force-probe simulations were three different structural capsid mutants of HRV 16. The first mutation was the removal of the pocket factor from the hydrophobic pocket in protein VP1. Docking of the ICAM-1 receptor to protein VP1 and emptying of the pocket was proposed to cause a destabilization of the capsid [131, 162, 61]. The suggested collapse of the structure was not observed after removal of the pocket factor and subsequent equilibration for $16 \mathrm{~ns}$. A possible reason for this observation is that destabilization is driven by the binding of the receptor to the capsid which was missing in the simulation system, and not by the absence of the pocket factor. Otherwise, the time scales of the simulations were too short to observe a possible closing of the pocket.

The HRV 16 structure includes 12 zinc ions located at the pentamer center and block a possible channel for RNA release along the 5-fold symmetry axis. If the removal of the zinc ions could cause a structural change of the capsid allowing the release of the RNA, remains unclear [61]. Additionally, the removal of protein VP4 was proposed as the first step in viral infection $[176,177]$. Therefore, the mechanical properties of the HRV 16 capsid without VP4 and zinc ions were studied in order to investigate changes in the elasticity and fracture behavior from which a possible port for RNA release could be proposed.

We obtained modifications in the mechanical properties on the inner and outer surfaces of the three HRV 16 capsid mutants compared to the initial structure. The largest changes of elastic constants and yielding forces were seen along the symmetry axes. Nevertheless, the pentamer center of all HRV 16 structure exhibited the smallest elastic constants and yielding forces, the 2-fold symmetry axis the largest stiffness and stability - only its absolute values changed with mutation of the capsid structure and were of the order of less than $1 \mathrm{~N} / \mathrm{m}$ and $1 \mathrm{nN}$, respectively. In contrast, these changes were much smaller as seen for SBMV after calcium removal.

The first step in cell infection with HRV 16 remains unclear. The question arises whether the pocket factor is removed first and then the ions, or is only the removal of protein VP4 necessary? Giranda et al. [27] proposed a possible pathway for RNA release along the 5-fold symmetry axis, similar to our suggestions for the RNA release of SBMV. The force-probe simulations clearly show that all HRV 16 mutants exhibit different mechanical properties, but the variations compared to the complete HRV 16 capsid were not strong enough to corroborate a possible pathway for 
RNA release through the pentamer center. Therefore, we suggest that additional mutations or combinations of the studied mutations are necessary to destabilize the capsid structure and result in a release of the RNA. In contrast to SBMV which becomes infections after removal of calcium in an alkaline environment, docking of external molecules, i.e. receptors to the capsid surface might also be necessary to cause RNA release of HRV 16.

From force-probe simulations in which the tip-sphere was pushed with probe-velocities between $0.001-0.05 \mathrm{~nm} / \mathrm{ps}$ along the 5-fold symmetry axis of SBMV, we found a logarithmic dependence for both elastic constants and yielding forces. Although such a velocity dependence of mechanical properties has already been observed earlier from deformation studies of metallic glasses [147], no theoretical model has been available until now to describe this peculiar behavior. A simple Kramers approach, i.e. the transition of a local energy barrier in a one-dimensional harmonic energy landscape which results in velocity independent elastic constant and logarithmic velocity dependent yielding forces [140, 141], is inadequate to describe our findings. Kramers theory, as well as more refined theories of enforces barrier crossings [142-146], rest on the assumption that all degrees of freedom perpendicular to the reaction coordinate are in equilibrium.

We therefore chose the ansatz that such a logarithmic behavior of elastic constants and yielding forces could be attributed to relaxation processes perpendicular to the reaction coordinate due to the high probe-velocities. This assumption was corroborated with Monte Carlo (MC) simulations in which a particle was pushed through a harmonic potential energy landscape with possible relaxation processes perpendicular to the reaction coordinate taking place. It turned out, that these relaxation dynamics resulted in a logarithmic velocity dependence of elastic constants and yielding forces and corroborated our force-probe simulation results very well.

The values of elastic constants and yielding forces obtained in experiments, were of the order of $0.5 \mathrm{~N} / \mathrm{m}$ and $0.5 \mathrm{nN}$, respectively. Extrapolating the logarithmic slope of the velocity dependent elastic constants and yielding forces to velocities of $1 \cdot 10^{-5} \mathrm{~m} / \mathrm{s}$ as used in AFM experiments [43] yielded elastic constant of $0.63 \mathrm{~N} / \mathrm{m}$ and yielding forces of $0.18 \mathrm{nN}$. These values are in good agreement with experimental findings [31].

Three possible influences and contributions to the obtained forces acting on the tipsphere were determined: (1) Frictional forces caused by the water molecules which interact with the tip-sphere, (2) forces acting on the inner capsid surface caused by an increase of the water pressure inside the capsid due to water compression, (3) increase of the surface free energy due to changes of the solvent hydrophobic surface area during indentation of the viral shell. The simulations showed that all three possibilities contribute to the yielding forces, obtained for SBMV and HRV 16, by approx. or less than $10 \%$.

Frictional forces are expected to contribute to the forces found in AFM experiments by much less than $10 \%$ because, although the AFM tip is about $10-20$ times larger 
than our tip-sphere, the approach velocity of the AFM tip is at least five orders of magnitude smaller as in our simulations [43]. An increase of the water pressure inside the capsid is not present in AFM experiments on empty viral shell and is in this sense an artifact. Besides, a change of the hydrophobic surface and thus a change in the surface free energy is also present in AFM experiments.

Water compression inside the capsids was obtained in the force-probe simulations because no water permeation through the viral shell was observed during indentations of SBMV and HRV 16. The non-existing water flux seems to be a special property of these viral shells because equilibration MD simulations of the fully solvated shell of Satellite Tobacco Necrosis Virus showed a large water flux [181]. Our observations agree with results by Silva et al. [125], who reported the only possibility for water flux through the shell of SBMV along the channel at the 5 -fold symmetry axis, whereas here a high energy barrier for water of $E=300 \mathrm{kcal} / \mathrm{mol}$ was obtained. They also proposed a similar water permeability and energy barrier at the pentamer center for Human Rhinoviruses which corroborates our observations.

Structural changes during deformation of the capsid were investigated. A highly elastic behavior of the viral shells was found, for which the $\beta$-sheet region behaved stiff compared to the $\alpha$-helices and loops. Such a behavior was already reported earlier for other proteins [128], whereas these results were obtained from experiments in which $\alpha$-helices and $\beta$-sheet regions were not indented but unfolded by pulling the C- and N-termina into opposite directions.

No propagation of deformation beyond the areas of the capsomer and subunit that were directly indented was seen during the force-probe simulations. Deformation occurred only locally on an area of a few $\mathrm{nm}^{2}$ that was directly touched by the tipsphere in contrast to AFM experiments in which the complete capsid was deformed. It still remains questionable if propagation of deformations into more distant regimes would be observed for slower probe-velocities when the capsid structure had more time to relax into more favorable positions during indentation. Nevertheless, the propagation behavior of deformation was very similar for all different probe-velocities between $0.001-0.05 \mathrm{~nm} / \mathrm{ps}$ due to the high elasticity of the coat protein. Thus, we deem a different deformation behavior with propagation to adjacent subunits of the indentation position unlikely even for velocities as used in AFM experiments. An important factor here is also the tip-sphere size which was at least ten times smaller than a typical AFM tip. Our simulations show that a larger tip-sphere resulted in an increase of the area of deformation. But also this larger tip-sphere was still much smaller than the diameter of a subunit and propagation of deformation to adjacent subunits was hardly observed for the employed probe-velocities. For this reason, also large indentation depths of the tip-sphere did not cause a buckling of the complete capsid which was only observed for deformations with indenting object of the size of at least one capsomer [22].

On the atomic level, the linear regime in the force-distance curves was characterized by a deformation of an otherwise topologically unchanged conformation of neighboring atoms. Elastic deformation was still seen in the sublinear rearrangement 
regime. Here structural changes were determined with marked distance changes between nearest and next-nearest neighbors. The higher force fluctuations in this regime were caused by recrossings of the system between the initial potential energy minimum and adjacent minima. The transition to plastic behavior was performed close to the yielding point, when a return of the system to the initial minimum was hampered. Only a few residues deformed plastically and fracture occurred locally at the position where the tip-sphere penetrated the viral shell. The remaining protein structure fully recovered when the tip-sphere left the capsid.

With this work we aim to contribute to a better understanding of the mechanical properties and their distribution on the inner and outer capsid surfaces of icosahedral viral shells. For the first time, structural dynamics and changes that determine the origin of elastic and plastic deformation during capsid indentation were investigated on atomic length scales. The simulations show that small changes of the capsid structure and thus changing electrostatic interactions of the shell atoms can result in significant changes of mechanical properties. From the variation of yielding forces due to structural mutations of the capsid, it is possible to propose a port in the shell structure that opens to release the RNA during cell infection. Nevertheless, open questions remain and a lot of work is left for the future for a complete understanding of viral assembly and dematuration. Some ideas for further investigations of the mechanical properties of viral shells with the force-probe simulation technique established in this work are given in the following chapter. 


\section{Outlook}

The following studies addressing the mechanical properties of viral shells could continue the present work. In order to study the swelling behavior of the Southern Bean Mosaic Virus capsid, the $\mathrm{pH}$ value of the system could be increased by changing the protonation states of the histidines in the protein shell because swelling was observed in experiments at $\mathrm{pH}=7.5-8.25$ [120]. To investigate structural changes of the mature shell of Human Rhinovirus 16, the ICAM-1 receptor could be modeled and docked to protein VP1. Additional removal of the fatty acid from the pocket in VP1 should result in a collapse of the empty pocket as seen in experiments. The resulting variation of mechanical properties on the capsid shell from which a possible scenario of RNA release might be derived, could than be studied with force-probe simulations.

Simulations with the same simulation technique could be performed on the bacteriophage $\phi$-X174 (PDB code: 2BPA). This icosahedral virus is $2 \mathrm{~nm}$ larger in diameter than SBMV and HRV 16 and contains DNA in contrast to the investigated RNA viruses. The larger persistence length of the DNA compared to RNA and, additionally, electrostatic interactions between the genetic material and the viral shell proteins result in high internal pressures. This high pressure inside the capsid could be employed to the simulation system, whereas the simulation of the DNA inside the virus is impossible because the structure is unknown, as well as the arrangement of the DNA inside the capsid. A modified pressure coupling could be programmed and implemented into GROMACS that allows the definition of pressure gradients inside the simulation box. With an increased water pressure inside the virus, its effect on the mechanical properties would be possible to determine.

In order to improve our model of the AFM tip that allows better comparison to AFM experiments, the shape and size of the tip-sphere could be changes. First, a tip-sphere with a larger diameter could be modeled. Second, the shape of the tip could be changed to pyramidal which could be modeled from several carbon atoms. An alternative tip-shape would be a mono-atomic or bi-atomic plate with several nanometers in diameter which is pressed against the viral surface. In comparison to a large spherical of pyramidal tip, here no thicker water shell is required to place this "plate" in the bulk water before it is pushed against the capsid, although the size of the indenting object is markedly increased compared to the tip-sphere used in our simulations.

A challenging project would be the calculation of the elastic constants of each capsid amino acid and even every atom from their thermal fluctuations, kinetic energies and the Born term, extracted from the force field [182, 183]. Deformation and indentation of the capsid are not necessary anymore and only equilibration simulations 
would have to be performed. From the monitored thermal fluctuations of the atoms during the equilibrium simulations, the whole elasticity tensor can directly be extracted from the sum of the Born term and a term accounting for internal relaxations $[183,184]$. Ray and Lutsko introduced a general description how the elasticity tensor, calculated from the strain derivatives of every atom during thermal fluctuations, can be derived from a potential/force field using computer simulations $[182,185]$. They only obtained fluctuation formulas for a pair potential, whereas their described formalism has already been expanded and applied for embedded-atom (EAM) potentials [186]. Challenging would be a generalization and implementation of this method for biomolecular systems and the OPLS force-field. Especially the description and calculations of long-range electrostatic interactions within this method are not straight forward. Although a great effort would be needed to carry out this project, the calculation of the elastic constants on atomic length scales offers the opportunity to directly investigate possible changes of mechanical behavior due to structural variations of the capsid and the removal of single atoms from the viral shell. 


\section{Acknowledgments}

Zum Schluss möchte ich all denen danken, die zu dieser Arbeit beigetragen haben. Vielen, vielen Dank an meinen Betreuer Prof. Dr. Helmut Grubmüller, der mir die Möglichkeit gegeben hat an seinem Institut meine Doktorarbeit zu diesem tollen Thema durchführen zu dürfen! Schließlich ist dies nicht eine normale Doktorarbeit, sondern der Weltrekord im Simulieren des größten biomolekularen Systems! Obwohl ich Helmut mit Sicherheit das ein oder andere Mal an den Rand des Wahnsinns getrieben habe, hat er mich nie aufgegeben, hatte Geduld und mir neben dem Wissen über Biophysik vor allem beigebracht, wie man Papers schreibt und dabei alle möglichen Referees gleichzeitig besänftigt.

Prof. Dr. R. Kirchheim danke ich für sein Interesse an meiner Arbeit und das Koreferat.

Ganz besonderer Dank gilt allen aktuellen und bereits ausgeschiedenen Mitgliedern der gesamten Arbeitsgruppe am Max-Planck-Institut, die mich von Beginn an herzlich aufgenommen haben. Insbesondere danke ich Dr. Carsten Kutzner für die Programmierung des Pull-Codes, Dr. Jürgen Haas, Dr. Martin Stumpe, Dr. Jochen Hub und Jürgen Lampe für ihre Bemühungen und Geduld mir das Skipten beizubringen. Vielen Dank an Dr. Nicole Dölker und Christian Kappel für die Zusammenarbeit am Projekt über die Geschwindigkeitsabhängigkeit der mechanischen Eigenschaften und insbesondere an Christian für die Programmierung des MC Codes, Dr. Maik Goette für seine permanente Flucherei, die mich immer wieder aufgebaut hat, Dr. Jürgen Haas, Dr. Lars Schäfer, Dr. Matthias Müller, Dr. Gerrit Groenhof und Dr. Daniel Seeliger für technische Hilfe, Dr. Ansgar Aestermann und Martin Fechner für die Instandhaltung(-setzung) meines Arbeitsplatzrechners und der Computer Cluster, und Dr. Ulrike Gerischer für das Korrekturlesen meiner Arbeit.

Dem Leibniz-Rechenzentrum München danke ich für 1.6 Mio. CPU-Stunden Rechenzeit, ohne die solch aufwenige Simulationen nicht möglich gewesen wären.

Henrike möchte ich danken für die ganzen Mittagessen, für die ich mich immer gerne von der Arbeit entfernt habe.

Mein größter Dank gilt meinen Eltern, die mich mein Leben lang unterstützt, mir vertraut und gut zugeredet haben und mich bis heute fragen, wofür das denn alles eigentlich gut ist.

Herzlichen Dank an Stefan, den ich permanent verrückt gemacht habe, weil angeblich nichts funktioniert und der mich immer wieder aufgebaut hat. Erst durch seine Unterstützung und "mitfühlenden Worte" ist mir bewusst geworden, dass ein $\mathrm{PhD}$ etwas tolles aber völlig unwichtige ist und andere Werte zählen. 


\section{Bibliography}

[1] N. H. Acheson. Fundamentals of molecular virology. John Wiley \& Sons, Inc., 2006.

[2] J. Carter and V. Saunders. Virology: Principles and applications. Wiley, 2007.

[3] M. G. Rossmann, C. Abad-Zapatero, and M. R. N. Murthy. Structural comparisons of some small spherical plant viruses. J. Mol. Biol., 165:711-736, 1983.

[4] K. W. Adolph and P. J. Butler. Assembly of a spherical plant virus. Philos. Trans. R. Soc. London B, 276:113-122, 1976.

[5] D. C. Reanney. The evolution of RNA viruses. Ann. Rev. Microbiol., 36:47-73, 1982.

[6] A. Schneemann. The structural and functional role of RNA in icosahedral viral assembly. Annu. Rev. Micromol., 60:51-67, 2006.

[7] A. Evilevitch, L. Lavelle, C. M. Knobler, E. Raspaud, and W. M. Gelbart. Osmotic pressure inhibition of DNA ejection from phage. Proc. Natl. Acad. Sci., 100:9292-9295, 2003.

[8] W. Jiang, J. Chang, J. Jakana, P. Weigele, J. King, and W. Chiu. Structure of $\epsilon 15$ bacteriophage reveals genome organization and DNA packaging/injection apparatus. Nature, 439:612-616, 2006.

[9] P. K. Purohit, M. M. Inamdar, P. D. Grayson, T. M. Squires, J. Kondev, and R. Phillips. Forces during bacteriophage DNA packaging and ejection. Biophys. J., 88:851-866, 2005.

[10] C. Forrey and M. Muthukumar. Langevin dynamics simulations of genome packing in bacteriophages. Biophys. J., 91:25-41, 2006.

[11] D. G. Angelescu, R. Bruisma, and P. Linse. Monte Carlo simulations of polyelectrolytes inside viral capsids. Phys. Rev. E, 73:041921, 2006.

[12] D. E. Smith, S. J. Tans, S. B. Smith, S. Grimes, D. L. Anderson, and C. Bustamante. The bacteriophage $\phi 29$ portal motor can package DNA against a large internal force. Nature, 413:748-752, 2001. 
[13] A. Cordova, M. Deserno, W. M. Gelbart, and A. Ben-Shauly. Osmotic shock and the strength of viral capsids. Biophys. J., 85:70-74, 2003.

[14] I. Ivanovska, G. Wuite, B. Joensson, and A. Evilevitch. Internal DNA pressure modifies stability of WT phage. Proc. Natl. Acad. Sci., 104:9603-9608, 2007.

[15] Z. Li, J. Wu, and Z.-G. Wang. Osmotic pressure and packaging structure of caged DNA. Biophys. J., 94:737-746, 2008.

[16] http://www.flutrackers.com.

[17] W. Chiu and J. Johnson, editors. Advances in Protein Chemistry, Volume 64: Virus Structure. Academic Press, 2003.

[18] M. Manchester and N. F. Steinmetz. Current Topics in Microbiology and Immunology: Viruses and Nanotechnology. Springer, Berlin, 2008.

[19] M. F. Hagan and D. Chandler. Dynamic pathways for viral capsid assembly. Biophys. J., 91:42-54, 2006.

[20] T. Douglas and M. Young. Virus particles as templates for materials sythesis. Adv. Mater., 8:11, 1999.

[21] H. Kasuya, S. Takeda, S. Nomoto, and A. Nakao. The potential of oncolytic virus therapy for pancreatic cancer. Cancer Gene Therapy, 12:725-736, 2005.

[22] W. S. Klug, R. F. Bruinsma, J.-P. Michel, C. M. Knobler, I. L. Ivanovska, C. F. Schmidt, and G. J. L. Wuite. Failure of viral shells. Phys. Rev. Lett., 97:228101, 2006.

[23] S. A. Shields, M. J. Brisco, T. M. A. Wilson, and R. Hull. Southern bean mosaic virus RNA remains associated with swollen virions during translation in wheat germ cell-free extracts. Virology, 171:602-606, 1989.

[24] H. Liu, C. Qu, J. E. Johnson, and D. A. Case. Pseudo-atomic models of swollen CCMV from cryo-electron microscopy data. J. Struct. Biol., 142:356363, 2002.

[25] F. Tama and C. L. Brooks III. Exploring large-scale conformational changes in virus maturation. J. Mol. Biol., 318:733-747, 2002.

[26] F. Tama and C. L. Brooks III. Diversity and identity of mechanical properties of icosahedral viral capsids studied with elastic network normal mode analysis. J. Mol. Biol., 345:299-314, 2005.

[27] V. L. Giranda and R. R. Rueckert. Acid-induced structural changes in human rhinovirus 14: possible role in uncoating. Proc. Natl. Acad. Sci., 89:1021310217, 1992. 
[28] A. G. Mosser, J.-Y. Sgro, and R. R. Rueckert. WIN51711-resistant mutants of poliovirus type 3: capsid residues important in uncoating functions. Acta Cryst. D, 51:490-495, 1995.

[29] N. Kol, M. Gladnikoff, D. Barlam, R. Z. Shneck, A. Rein, and I. Rousso. Mechanical properties of murine leukemia virus particles: Effect of maturation. Biophys. J., 91:767-774, 2006.

[30] R. Zandi, D. Reguera, R. F. Bruinsma, W. M. Gelbart, and J. Rudnick. Origin of icosahedral symmetry in viruses. Proc. Nat. Acad. Sci., 101:15556-15560, 2004.

[31] W. H. Roos, I. L. Ivanovska, A. Evilevitch, and G. J. L. Wuite. Viral capsids: mechanical characteristics, genome packaging and delivery mechanisms. Cell. Mol. Life Sci., 64:1484-1497, 2007.

[32] G. Binnig, C. F. Quate, and Ch. Gerber. Atomic force microscopy. Phys. Rev. Lett., 56:930-933, 1986.

[33] N. C. Santos and M. A. R. B. Castanho. An overview of the biophysical applications of atomic force microscopy. Biophys. Chem., 107:133-149, 2004.

[34] Y. G. Kuznetsov, S. Daijogo, J. Zhou, B. L. Semler, and A. McPherson. Atomic force microscopy analysis of icosahedral virus RNA. J. Mol. Biol., 347:41-52, 2007.

[35] Y. L. Lyobschenko, P. I. Oden, D. Lampner, S. M. Lindsay, and K. A. Dunker. Atomic force microscopy of DNA and bacteriophage in air, water and propanol: The role of adhesion forces. Nucleic Acids Res., 21:1117-1123, 1993.

[36] D. Anselmetti, M. Dreier, R. Lüthi, T. Richmond, E. Meyer, J. Frommer, and H.-J. Güntherodt. Biological materials studied with dynamic force microscopy. J. Vac. Sci. Technol. B, 12:1500-1503, 1994.

[37] F. M. Ohnesorge, J. K. H. Härber, W. Häberle, C.P. Czerny, D. P. E. Smith, and G. Binnig. AFM review study on pox viruses and living cells. Biophys. J., 73:2183-2194, 1997.

[38] Y. G. Kuznetsov, A. J. Malkin, R. W. Lucas, M. Plomp, and A. McPherson. Imaging of viruses by atomic force microscopy. J. Gen. Virol., 82:2025-2034, 2001.

[39] A. J. Malkin, Y. G. Kuznetsov, and A. McPherson. Viral capsomere structure, surface processes and growth kinetics in the crystallization of macromolecular crystals visualized by in situ atomic force microscopy. J. Cryst. Growth, 232: 173-183, 2001. 
[40] M. Plomp, M. K. Rice, E. K. Wagner, A. McPherson, and A. J. Malkin. Rapid visualization at high resolution of pathogens by atomc force microscopy: Structural studies of herpes simplex virus-1. Am. J. Pathol., 160:1959-1966, 2002.

[41] Y. G. Kuznetsov, J. R. Gurnon, J. L. Van Etten, and A. McPherson. Atomic force microscopy investigation of a chlorella virus, PBCV-1. J. Struc. Biol., 149:256-263, 2005.

[42] M. R. Falvo, S. Washburn, R. Superfine, M. Finch, Jr. F. P. Brooks, V. Chi, and R. M. Taylor. Manipulation of individual viruses: friction and mechanical properties. Biophys. J., 72:1396-1403, 1997.

[43] I. L. Ivanovska, P. J. de Pablo, B. Ibarra, G. Sgalari, F. C. MacKintosh, J. L. Carrascosa, C. F. Schmidt, and G. J. L. Wuite. Bacteriophage capsids: Tough nanoshells with complex elastic properties. Proc. Natl. Acad. Sci., 101:7600$7605,2004$.

[44] C. Carrasco, A. Carreira, I. A. T. Schaap, P. A. Serena, J. Gomez-Herrero, M. G. Mateu, and P. J. de Pablo. DNA-mediated anisotropic mechanical reinforcement of a virus. Proc. Natl. Acad. Sci., 103:13706-13711, 2006.

[45] A. Schmatulla, N. Maghelli, and O. Marti. Micromechanical properties of tobacco mosaic viruses. J. Microscopy, 225:264-268, 2007.

[46] N. Kol, Y. Shi, D. Barlam, R. Z. Shneck, M. S. Kay, and I. Rousso. A stiffness switch in human immunodeficiency virus. Biophys. J., 92:1777-1783, 2007.

[47] B. M. Hespenheide, D. J. Jacobs, and M. F. Thorpe. Structural rigidity in the capsid assembly of cowpea chlorotic mottle virus. J. Phys. Condens. Matter, 16:S5055-S5064, 2004.

[48] R. Zandi and D. Reguera. Mechanical properties of viral capsids. Phys. Rev. E, 72:021917, 2005.

[49] M. Widom, J. Lidmar, and D. R. Nelson. Soft modes near the buckling transition of icosahedral shells. Phys. Rev. E, 76:031911, 2007.

[50] G. A. Vliegenthart and G. Gompper. Mechanical deformation of spherical viruses with icosahedral symmetry. Biophys. J., 91:834-841, 2006.

[51] F. Gräter, J. Shen, H. Jiang, M. Gautel, and H. Grubmüller. Mechanically induced titin kinase activation studied by force-probe molecular dynamics simulations. Biophys. J., 88:790-804, 2005.

[52] P. E. Marszalek, H. Lu, H. Li, M. Carrion-Vazquez, A. F. Oberhauser, K. Schulten, and J. M. Fernandez. Mechanical unfolding intermediates in titin modules. Nature, 402:100-103, 1999. 
[53] P. L. Freddolino, A. S. Arkhipov, S. B. Larson, A. McPherson, and K. Schulten. Molecular dynamics simulations of the complete satellite tobacco mosaic virus. Structure, 14:437-449, 2006.

[54] D. K Phelps, P. J. Rossky, and C. B. Post. Influence of an antiviral compound on the temperature dependence of viral protein flexibility and packing: A molecular dynamics study. J. Mol. Biol., 276:331-337, 1998.

[55] B. Speelman, B. R. Brooks, and C. B. Post. Molecular dynamics simulations of human rhinovirus and an antiviral compound. Biophys. J., 80:121-129, 2001.

[56] S. Yoneda, M. Kitazawa, and H. Umeyama. Molecular dynamics simulations of a rhinovirus capsid under rotational symmetry boundary conditions. $J$. Comp. Chem., 17:191-203, 1995.

[57] S. Yoneda, T. Yoneda, Y. Kurihara, and H. Umeyama. Motion of an antiviral compound in a rhinovirus capsid under rotational symmetry boundary conditions. J. Molecular Graphics and Modelling, 21:19-27, 2002.

[58] H. Grubmüller, B. Heymann, and P. Tavan. Ligand binding: Molecular mechanics calculation of the streptavidin-biotin rupture force. Science, 271:997999, 1996.

[59] B. Heymann and H. Grubmüller. Molecular dynamics force probe simulations of antibody/antigen unbinding: Entropic control and nonadditivity of unbinding forces. Biophys. J., 81:1295-1313, 2001.

[60] I. Rayment, J. E. Johnson, and M. G. Rossmann. Metal-free southern bean mosaic virus crystals. J. Biol. Chem., 254:5243-5245, 1979.

[61] A. T. Hadfield, W.-M. Lee, R. Zhao, M. A. Oliveira, I. Minor, R. R. Rueckert, and M. G. Rossmann. The refined structure of human rhinovirus 16 at $2.15 \AA$ resolution: implifications for the viral life cycle. Structure, 5:427-441, 1997.

[62] A. P. Waterson. The significance of viral structure. Archive Virol., 15:275-300, 1965.

[63] B. Berger, J. King, R. Schartz, and P. W. Shor. Local rule mechanism for selecting icosahedral shell geometry. Disc. Appl. Math., 104:97-111, 2000.

[64] J. D. Watson and F. H. C. Crick. Genetical implications of the structure of deoxyribonucleic acid. Nature, 171:964-967, 1953.

[65] J. D. Watson and F. H. C. Crick. Molecular structure of nucleic acids: A structure for deoxyribose nucleic acid. Nature, 171:737, 1953.

[66] F. H. C. Crick and J. D. Watson. Structure of small viruses. Nature, 177: 473-475, 1956. 
[67] http://de.wikipedia.org/wiki/kapsid.

[68] S. Brenner and R. W. Horne. A negative staining methode for high resolution electron microscopy of viruses. Biochem. Biophys. Acta, 34:103-110, 1959.

[69] A. Lwoff, T. F. Anderson, and F. Jacob. Remarks on the characteristics of the infectious viral particle. Ann. Inst. Pasteur (Paris), 97:281-289, 1959.

[70] R. W. Horne, S. Brenner, A. P. Waterson, and P. Wildy. The icosahedral form of an adenovirus. J. Mol. Biol., 1:84-86, 1959.

[71] H. E. Huxley and G. Zubay. Structure of the protein shell of turnip yellow mosaic virus. J. Mol. Biol., 1:189-196, 1960.

[72] http://www.sachpa.com/herpesvirus-info-16.html.

[73] D. L. D. Caspar and A. Klug. Physical principles in the construction of regular viruses. Cold Spring Harbor Symp. on Quant. Biol., 27:1-50, 1962.

[74] R. W. Horne and P. Wildy. Symmetry in virus architecture. Virology, 15: 348-373, 2006.

[75] R. E. Franklin. Structure of tobacco mosaic virus. Nature, 175:379-381, 1955.

[76] M. G. Rossmann, E. Arnold, J. P. Griffith, G. Kramer, M. Luo, T. J. Smith, G. Vried, R. R. Rueckert, B. Sherry, M. A. McKinlay, G. Diana, and M. Otto. Common cold viruses. TIBS, 12:313-318, 1987.

[77] F. B. Bang. Studies on Newcastle disease virus. III. characters of the virus itself with particular reference of electron microscopy. J. Exp. Medicine, 88: 251-266, 1948.

[78] V. J. Cabasso. The emerging classification of animal viruses: A review. Avian Diseases, 9:471-489, 1965.

[79] D. L. D. Caspar, R. Dulbecco, A. Klug, A. Lwoff, M. G. P. Stoker, P. Tournier, and P. Wildy. Proposals. Cold Spring Harbor Symposia on Quant. Biol., 27: 49-50, 1962.

[80] S. C. Harrison, A. J. Olson, C. E. Schutt, F. K. Winkler, and G. Bricogne.

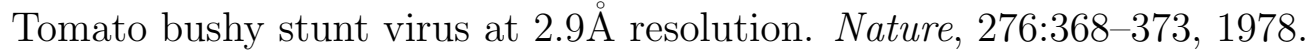

[81] A. M. Silva and M. G. Rossmann. The refinement of southern bean mosaic virus in reciprocal space. Acta. Cryst. B, 41:147-157, 1985.

[82] L. Liljas, T. Unge, T. A. Jones, K. Fridborg, S. Lövgren, U. Skoglund, and B. Strandberg. The structure of satellite tobacco necrosis virus at 3.0A resolution. J. Mol. Biol., 159:93-108, 1982. 


\section{Bibliography}

[83] VirusWorld. virology.wisc.edu/cgi-bin/virusworld/htdocs.pl?docname=triangulation.html.

[84] M. Born and R. Oppenheimer. Zur Quantentheorie der Molekeln. Ann. Phys. Leipzig, 84:457, 1927.

[85] F. C. Brooks. Convergence of intermolecular force series. Phys. Rev., 86:92-97, 1952.

[86] R. B. Gerber. On the order of accuracy of the Born-Oppenheimer approximation for molecular collision states. Proc. Roy. Soc. London. Ser. A - Math. and Phys., 309:221-244, 1969.

[87] W. L. Jorgensen and J. Tirado-Rives. The OPLS potential function for proteins. energy minimization of cyclic peptides and crambin. J. Am. Chem. Soc, 110:1657-1666, 1988.

[88] U. Dinur and A. T. Hagler, editors. New approaches to empirical force fields. Wiley, 1991.

[89] W. L. Jorgensen and J. Tirado-Rives. Potential energy functions for atomiclevel simulations of water and organic and biomolecular systems. Proc. Natl. Acad. Sci., 102:6665-6670, 2005.

[90] L. Verlet. Computer experiments on classical fluids .i. thermodynamical properties of Lennard-Jones molecules. Phys. Rev., 159:98-103, 1967.

[91] B. Hess, H. Bekker, H. J. C. Berendsen, and J. G. E. M. Fraaije. LINCS: a linear constraint solver for molecular simulations. J. Comput. Chem., 18: 1463-1472, 1997.

[92] H. J. L. Berendsen, J. P. M. Postma, W. F. van Gunsteren, A. D. Nola, and J. R. Haak. Molecular dynamics with coupling to an external bath. J. Chem. Phys., 81:3684-3690, 1984.

[93] Wolfram Mathworld. http://mathworld.wolfram.com/rhombicdodecahedron.html.

[94] GROMACS-3.3.1 User-Manual. www.gromacs.org.

[95] H. Bekker, E. J. Dijkstra, M. K. R. Renardus, and H. J. C. Berendsen. An efficient, box shape independent non-bonded force and virial algorithm for molecular dynamics. Mol. Sim., 14:137-152, 1995.

[96] D. van der Spoel, E. Lindahl, B. Hess, G. Groenhof, A. E. Mark, and H. J. C. Berendsen. GROMACS: Fast, flexible, and free. J. Comput. Chem., 26:17011718, 2005.

[97] W. L. Jorgensen, J. Chandrasekhar, J. D. Madura, R. W. Impey, and M. L. Klein. Comparison of simple potential functions for simulating liquid water. J. Chem. Phys., 79:926-935, 1983. 
[98] V. S. Reddy, P. Natarajan, B. Okerberg, K. Li, K. V. Damodaran, R. T. Morton, C. L. Brooks III, and J. E. Johnson. Virus Particle Explorer (VIPER), a webside of viral capsid structures and their computational analyses. J. Virol., 75:11943-11947, 2001.

[99] P. Natarajan, G. C. Lander, C. M. Shepherd, V. S. Reddy, C. L. Brooks, and J. E. Johnson. Exploring icosahedral virus structures with VIPER. Nature, 3: 809-817, 2005.

[100] G. Vried. WHAT IF: A molecular modeling and drug design program. J. Mol. Graph., 8:52-56, 1990.

[101] T. Darden, D. York, and L. Pedersen. Particle mesh Ewald - an N $\log (\mathrm{N})$ method for Ewald sums in large systems. J. Chem. Phys., 98:10089-10092, 1993.

[102] C. Kutzner, D. van der Spoel, M. Fechner, E. Lindahl, U. W. Schmitt, B. L. de Groot, and H. Grubmüller. Speeding up parallel GROMACS on highlatency networks. J. Comp. Chem., 28:2075-2084, 2007.

[103] B. Hess, C. Kutzner, D. van der Spoel, and E. Lindahl. GROMACS 4: Algorithms for highly efficient, load-balanced, and scalable molecular simulation. J. Chem. Theory Comput., 4:435-447, 2008.

[104] W. Humphrey, A. Dalke, and K. Schulten. VMD - Visual Molecular Dynamics. J. Molec. Graphics, 14:33-38, 1996.

[105] L. Hoehn and I. Niven. Averages on the move. Math. Mag., 58:151-156, 1985.

[106] K.-Q. Mang, A. Ghosh, and P. Kaesberg. A comparative study of the cowpea and bean strains of southern bean mosaic virus. Virology, 116:264-274, 1982.

[107] R. J. Shepherd and R. W. Fulton. Identity of seed-borne virus of cowpea. Phytophathology, 52:489-493, 1962.

[108] C. Abad-Zapatero, S. S. Abdel-Meguid, J. E. Johnson, A. G. W. Leslie, I. Rayment, M. G. Rossmann, D. Suck, and T. Tsukihara. Structure of southern bean mosaic virus at $2.8 \AA$ resolution. Nature, 286:33-39, 1980.

[109] http://comp.uark.edu/ morelock/cowpea_virus.html.

[110] H. S. Savithri and J. W. Erickson. The self-assemply of the cowpea strain of southern bean mosaic virus: Formation of $\mathrm{T}=1$ and $\mathrm{T}=3$ nucleoprotein particles. Virology, 126:328-335, 1983.

[111] A. M. Silva and M. G. Rossmann. Refined structure of southern bean mosaic virus at 2.9A resolution. J. Mol. Biol., 197:69-87, 1987. 
[112] B. S. Magdoff. Sub-units in southern bean mosaic virus. Nature, 4714:673-674, 1960.

[113] M. A. Hermodson, C. Abad-Zapatero, S. S. Abdel-Meguid, S. Pundak, M. G. Rossmann, and J. H. Tremaine. Amino acid sequence of southern bean mosaic virus coat protein and its relation to the three-dimensional structure of the virus. Virology, 119:133-149, 1982.

[114] W. C. Price. Purification and crystallization of southern bean mosaic virus. American J. Botany, 33:45-54, 1946.

[115] M. G. Rossmann, C. Abad-Zapatero, M. A. Hermodson, and J. W. Erickson. Subunit interactions in southern bean mosaic virus. J. Mol. Biol., 166:37-83, 1983.

[116] S. S. Abdel-Meguid, T. Yamane, K. Fukuyama, and M. G. Rossmann. The location of calcium ions in southern bean mosaic virus. Virology, 114:81-85, 1981.

[117] C. H. Hsu, O. P. Sehgal, and E. E. Pickett. Stabilizing effect of divalent metal ions on virions of southern bean mosaic virus. Virology, 69:587-595, 1976.

[118] R. Hull. The stabilization of the particles of turnip rosette virus and other members of the southern bean mosaic virus group. Virology, 79:58-66, 1977.

[119] R. Hull. The stabilization of the particles of turnip rosette virus: III. divalent cations. Virology, 89:418-422, 1978.

[120] M. Brisco, C. Haniff, R. Hull, T. M. A. Wilson, and D. B. Sattelle. The kinetics of swelling of southern bean mosaic virus: A study using photon correlation spectroscopy. Virology, 148:218-220, 1986.

[121] R. E. F. Matthews and J. Witz. Uncoating of turnip yellow mosaic virus RNA in vitro. Virology, 144:318-327, 1985.

[122] R. Virudachalam, K. Sitaraman, K. L. Huess, J. L. Markley, and P. Argos. Evidence of $\mathrm{pH}$-induced release of RNA from belladonna mottle virus and the stabilizing effect of polyamines and cations. Virology, 130:351-359, 1983.

[123] T. M. A. Wilson. Cotranslational disassembly of tobacco mosaic virus in vitro. Virology, 137:255-265, 1984.

[124] M. J. Brisco, R. Hull, and T. M. A. Wilson. Southern bean mosaic virusspecific proteins are synthesized in an in vitro system supplemented with intact, treated virions. Virology, 143:392-398, 1985.

[125] A. M. Silva, R. E. Cachau, and D. J. Goldstein. Ion channels in southern bean mosaic virus capsid. Biophys. J., 52:595-602, 1987. 
[126] B. deGroot. private communications. 2008.

[127] J. P. Michel, I. L. Ivanovska, M. M. Gibbons, W. S. Klug, C. M. Knobler, G. J. L. Wuite, and C. F. Schmidt. Nanoindentation studies of full and empty viral capsids and the effects of capsid protein mutations on elasticity and strength. Proc. Natl. Acad. Sci., 103:6184-6189, 2006.

[128] T. Ackbarow, X. Chen, S. Keten, and M. J. Buehler. Hierarchies, multiple energy barriers, and robustness govern the fracture mechanics of $\alpha$-helical and $\beta$-sheet protein domains. Proc. Natl. Acad. Sci., 16:16410-16415, 2007.

[129] T. T. Nguyen, R. F. Bruinsma, and W. M. Gelbart. Elasticity theory and shape transitions of viral shells. Phys. Rev. E, 72:051923, 2005.

[130] V. S. Reddy, H. A. Giesing, R. T. Morton, A. Kumar, C. B. Post, C. L. Brooks, and J. E. Johnson. Energetics of quasiequivalence: Computational analysis of protein-protein interactions in icosahedral viruses. Biophys. J., 74:546-558, 2002.

[131] M. G. Rossmann, E. Arnold, J. W. Erickson, E. A. Frankenberger, J. P. Griffith, H.-J. Hecht, J. E. Johnson, G. Kamer, M. Luo, A. G. Mosser, R. R. Rueckert, and G. Vried. Structure of human cold virus and functional relationship to other picornaviruses. Nature, 317:145-153, 1985.

[132] W. L. Jorgensen and J. D. Madura. Temperature and size dependence for Monte Carlo simulations of TIP4P water. Mol. Phys., 56:1381-1392, 1985.

[133] D. Bertolini and A. Tani. Stress tensor and viscosity of water: Molecular dynamics and generalized hydrodynamics results. Phys. Rev E, 52:1699, 1995.

[134] L. Bocquet and J.-L. Barrat. Hydrodynamic boundary conditions, correlation functions, and Kubo relations for confined fluids. Phys. Rev. E, 49:3079-3092, 1993.

[135] J. R. Schmidt and J. L. Skinner. Hydrodynamic boundary conditions, the Stokes-Einstein law, the long-time tails in the Brownian limit. J. Chem. Phys., 119:8062-8068, 2003.

[136] J. A. Reynolds, D. B. Gilbert, and C. Tanford. Empirical correlation between hydrophobic free energy and aqueous cavity surface area. Proc. Natl. Acad. Sci., 71:2925-2927, 1974.

[137] T. M. Raschke, J. Tsai, and M. Levitt. Quantification of the hydrophobic interaction by simulations of the aggregation of small hydrophobic solutes in water. Proc. Natl. Acad. Sci., 98:5965-5969, 2001.

[138] R. Merkel, P. Nassoy, A. Leung, K. Ritchie, and E. Evans. Energy landscapes of receptor-ligand bonds explored with dynamic force spectroscopy. Nature, 397:50-53, 1999. 
[139] E. Puchner, A. Alexandrovitch, B. Brandmeier, U. Hensen, L. Schäfer, F. Gräter, H. Grubmüller, H. Gaub, and M. Gautel. Mechanically activated ATP binding of the titin kinase domain revealed by single-molecule force spectroscopy. Proc. Natl. Acad. Sci., 105:13385-13390, 2008.

[140] H. A. Kramers. Brownian motion in a field of force and the diffusion model of chemical reactions. Physica, 7:284-304, 1940.

[141] G. I. Bell. Models for the specific adhesion of cells to cells. Science, 200: 618-627, 1978.

[142] E. L. Florin, V. T. Moy, and H. E. Gaub. Ligand binding: molecular mechanics calculation of the streptavidin-biotin rupture force. Science, 264:415-417, 1994.

[143] B. Heymann and H. Grubmüller. Dynamic force spectroscopy of molecular adhesion bonds. Phys. Rev. Lett., 84:6126-6128, 2000.

[144] G. Hummer and A. Szabo. Kinetics from nonequilibrium single-molecule pulling experiments. Biophys. J., 85:5-15, 2003.

[145] M. Evstigneev and P. Reimann. Rate description in friction force microscopy. Europhys. Lett., 67:907-913, 2004.

[146] E. A. Evans and D. A. Calderwood. Forces and bond dynamics in cell adhesion. Science, 316:1148-1153, 2007.

[147] M. Zink, K. Samwer, W. L. Johnson, and S. G. Mayr. Validity of temperature and time equivalence in metallic glasses during shear deformation. Phys. Rev. B, 74:012201, 2006.

[148] V. V. Hamparian, R. J. Colonno, M. K. Cooney, E. C. Dick, J. M. Jr. Gwaltney, J.H. Hughes, W. S. Jr. Jordan, A. Z. Kapikian, W. J. Mogabgab, A. Monto, C. A. Phillips, R. R. Rueckert, J. H. Scheible, E. J. Stott, and D. A. J. Tyrrell. A collaboration report: rhinoviruses-extention of the numbering system from 89 to 100. Virology, 159:191-192, 1987.

[149] T. C. Mettenleiter and F. Sobrino, editors. Animal viruses: Molecular biology. Caister Academic Press, 2008.

[150] R. R. Rueckert and E. Wimmer. Systematic nomenclature of picornavirus proteins. J. Virol., 50:957-959, 1984.

[151] M. A. Pallansch, O. W. Kew, B. L. Semler, D. R. Omilianowski, C. W. Anderson, E. Wimmer, and R. R. Rueckert. Protein processing map of poliovirus. J. Virol., 49:873-880, 1984.

[152] G. Abraham and R. J. Colonno. Many rhinovirus serotypes share the same cellular receptor. J. Virol., 51:340-345, 1984. 
[153] C. R. Uncapher, C. M. DeWitt, and R. J. Colonno. The major and minor group receptor families contain all but one human rhinovirus serotype. Virology, 180: 814-817, 1991.

[154] M. G. Rossmann, Y. He, and R. J. Kuhn. Picornavirus-receptor interactions. Trends Microbiol., 10:324-331, 2002.

[155] R. S. Polin, M. Bavbek, M. E. Shaffrey, K. Billups, C. A. Bogaev, N. F. Kassell, and K. S. Lee. Detection of soluble E-selectin, ICAM-1, VCAM-1 and L-selectin in the cerebrospinal fluid of patients after subarachnoid hemorrhage. J. Neurosurgery, 89:559-567, 1998.

[156] J. Bella and M. G. Rossmann. Review: Rhinovirus and their ICAM receptors. J. Struct. Biol., 128:69-74, 1999.

[157] B. A. Heinz, R. R. Rueckert, D. A. Shepard, F. J. Dutko, M. A. McKinlay, M. Fancher, M. G. Rossmann, J. Badger, and T. J. Smith. Genetic and molecular analyses of spontaneous mutants of human rhinovirus 14 that are resistant to an antiviral compound. J. Virol., 63:2476-2485, 1989.

[158] Y. Li, Z. Zhou, and C. B. Post. From the cover: Dissociation of an antiviral compound from the internal pocket of human rhinovirus 14 capsid. Proc. Natl. Acad. Sci., 102:7529-7534, 2005.

[159] J. E. Tomassini and R. J. Conollo. Isolation of a receptor protein involved in attachment of human rhinoviruses. J. Virol., 58:290-295, 1986.

[160] J. M. Greve and A. McClelland. The major human rhinovirus receptor is ICAM-1. Cell, 56:839-847, 1989.

[161] D. E. Staunton, V. L. Merluzzi, R. Rothlein, R. Barton, S. D. Marling, and T. A. Springer. A cell adhesion molecule, ICAM-1, is the major surface receptor for rhinoviruses. Cell, 56:849-853, 1989.

[162] M. A. Oliveira, R. Zhao, W.-M. Lee, M. J. Kremer, I. Minor, R. R. Rueckert, G. D. Diana, D. C. Pevear, F. J. Dutko, M. A. McKinley, and M. G. Rossmann. The structure of human rhinovirus 16. Structure, 1:51-68, 1993.

[163] K. H. Kim, T. J. Smith, M. S. Chapman, M. G. Rossmann, D. C. Pevear, F. J. Dutko, P. J. Felock, G. D. Diana, and M. A. McKinlay. Crystal structure of human rhinovirus serotype 1a (HRV1A). J. Mol. Biol., 210:91-111, 1989.

[164] K. H. Kim, P. Willingmann, Z. X. Gong, M. J. Kremer, M. S. Chapman, I. Minor, M. A. Oliveira, M. G. Rossmann, K. Andries, G. D. Diana, F. J. Dutko, M. A. McKinlay, and D. C. Pevear. A comparison of the anti-rhinoviral drung binding pocket in HRV14 and HRV1A. J. Mol. Biol., 230:206-227, 1993. 
[165] A. C. Palmenberg. Sequence alignments of picornaviral capsid proteins. American Society of Microbiology, 1989. Molecular aspects of picornavirus infection and detection.

[166] R. F. Lemanske, E. C. Dick, C. A. Swenson, and R. F. Vrtis. Rhinovirus upper respiratory infection increases airway hyperreactivity and late astmatic reactions. J. Clin. Invest., 83:1-10, 1989.

[167] M. G. Rossmann and J. E. Johnson. Icosahedral RNA virus structures. Annu. Rev. Biochem., 58:533-573, 1989.

[168] S. C. Harrison. Viruses. Curr. Opin. Struct. Biol., 2:293-299, 1992.

[169] M. G. Rossmann. The canyon hypothesis. hiding the host cell receptor attachment site on the viral surface from immune surveillance. J. Mol. Biol., 264: 14587-14590, 1989.

[170] M. G. Rossmann. The canyon hypothesis. Vir. Immunol., 2:143-161, 1989.

[171] Y. Zhang, A. A. Simpson, R. M. Ledford, C. M. Bator, S. Chakravarty, G. A. Skochko, T. M. Demenczuk, A. Watanyar, D. C. Pevear, and M. G. Rossmann. Structural and virological studies of the stages of virus replication that are affected by antirhinovirus compounds. J. Virol., 78:10034-10044, 2004.

[172] J. J. McSharry, L. A. Caliguira, and H. J. Eggers. Inhibition of uncoating of poliovirus by arildone, a new antiviral drug. Virology, 97:307-315, 1979.

[173] G. D. Diana and F. Pancic. Antiviral activity of some $\beta$-diketones. 1. aryl alkl diketones. in vitro activity against both RNA and DNA viruses. J. Med. Chem., 20:750-756, 1977.

[174] D. C. Pevear and F. J. Dutko. Conformational change in the floor of the human rhinovirus canyon blocks adsorption to HeLa cell receptors. J. Virol., 63:2002-2007, 1989.

[175] D. K Phelps and C. B. Post. A novel basis of capsid stabilization by antiviral compounds. J. Mol. Biol., 254:544-551, 1995.

[176] K. Lonberg-Holm and B. D. Korant. Early interaction of rhinoviruses with host cells. J. Virol., 9:29-40, 1972.

[177] L. Everaert, R. Vrijsen, and A. Boeyé. Eclipse products of poliovirus after cold-synchronized infection of HeLa cells. Virology, 171:76-82, 1989.

[178] R. Basavappa, R. Syed, O. Flore, J. P. Icenogle, D. J. Filman, and J. M. Hogle. Role and mechanism of the maturation cleavage of VP0 in poliovirus assembly: Structure of the empty capsid assembly intermediate at $2.9 \AA$ resolution. Protein Sci., 3:1651-1669, 1994. 
[179] A. Fiser and A. Sali. ModLOOP: automated modeling of loops in protein structures. Bioinformatics, 19:2500-2501, 2003.

[180] E. M. Puchner, A. Alexandrovich, A. L. Kho, U. Hensen, L. V. Schäfer, B. Brandmeier, F. Gräter, H. Grubmüller, H. E. Gaub, and M. Gautel. Mechanoenzymatics of titin kinase. Proc. Natl. Acad. Sci., 105:13385-13390, 2008.

[181] Daniel Larsson. The big picture: Simulation of a virus particle. GROMACS Workshop, Göttingen, 2008.

[182] J. R. Ray. Elastic constants and statistical ensembles in molecular dynamics. Comput. Phys. Rep., 8:109-151, 1988.

[183] J. F. Lutsko. Stress and elastic constants in anisotropic solids: Molecular dynamics techniques. J. Appl. Phys., 64:1152-1154, 1988.

[184] J. F. Lutsko. Statistical calculation of elastic moduli for atomistic models. Phys. Rev. B, 71:184108, 2005.

[185] J. F. Lutsko. Generalized expressions for the calculation of elastic constants by computer simulation. J. Appl. Phys., 65:2991-2997, 1989.

[186] S. G. Mayr. Relaxation kinetics and mechanical stability of metallic glasses and supercooled melts. Phys. Rev. B - in press, 2009. 


\section{Lebenslauf von Mareike Zink}

\section{Persönliche Daten}

Name:

Mareike Zink

Adresse:

Zur Hohen Warte 19, 37077 Göttingen

Geburtsdatum/-ort:

06.09.1979, Osterholz-Scharmbeck

Nationalität:

Deutsch

\section{Ausbildung}

06.1999

Abitur, Gymnasium Osterholz-Scharmbeck

08.1999-07.2001

Ausbildung zur Bankkauffrau bei der Dresdner Bank in Bremen und Hamburg und Trainee zum Investmentbanker bei Dresdner Kleinwort Wasserstein in Frankfurt am Main

$09.2001-05.2006$

Studium der Physik an der Georg-August Universität Göttingen, Abschluss: Diplom-Physikerin

$03.2005-03.2006$

Diplomarbeit am 1. Physikalischen Institut bei Prof. Dr. K. Samwer zum Thema: Mikroskopisches und makroskopisches Verhalten von CuTi Glas beim Scheren - Eine Simulationsstudie

$07.2006-03.2009$

Promotion in Physik am Max-Planck-Institut für biophysikalische Chemie, Abteilung für theoretische und computergestützte Biophysik bei Prof. Dr. H. Grubmüller 